\title{
LUDWIG-MAXIM!̣LIANS-UNIVERSITÄT MÜNCHEN
}

\author{
A New Generation \\ of Ultrafast Oscillators \\ for Mid-Infrared Applications
}

Nathalie Nagl

München 2020 



\title{
LUDWIG-MAXIMILIANS-UNIVERSITÄT MÜNCHEN
}

\section{A New Generation of Ultrafast Oscillators for Mid-Infrared Applications}

\author{
Dissertation \\ an der Fakultät für Physik \\ der Ludwig-Maximilians-Universität \\ München
}

vorgelegt von

Nathalie Nagl

aus Landshut

München, 30. Oktober 2020 

Erstgutachter: Prof. Dr. Ferenc Krausz Zweitgutachter: Prof. Dr. Ursula Keller Tag der mündlichen Prüfung: 21. Januar 2021 

Für meine Eltern und Großeltern, für meine Geschwister, und für Markus. 



\section{Zusammenfassung}

Der mittelinfrarote (MIR) Spektralbereich, der die Wellenlängen zwischen $2 \mu \mathrm{m}$ und $20 \mu \mathrm{m}$ umspannt, birgt enormes Potential für die Untersuchung komplexer biologischer Systeme, insbesondere angesichts der großen Anzahl an detektierbaren intensiven und einzigartigen molekularen Absorptionslinien. Spektroskopische Anwendungen, die sich Strahlung im mittleren Infrarot bedienen, sind deshalb in den letzten Jahren in den Fokus der Forschung gerückt. Ein besonders eindrucksvolles Beispiel ist die Kombination von gepulsten Lichtquellen mit Repetitionsraten mehrerer zehn Megahertz und Impulsdauern von wenigen optischen Zyklen mit feldaufgelösten Techniken. Letztere ermöglichen es, molekulare Signale nach Amplitude und Phase aufgelöst mit einer Empfindlichkeit am Quantenrauschen zu detektieren. Trotz des stetig wachsenden Forschungsbedarfs erschwert der Mangel an rauscharmen, kompakten und zugleich gepulsten Lasersystemen deren breite Anwendung.

In dieser Dissertation wird eine neue Generation von kompakten MittelinfrarotLaserquellen präsentiert. Diese vereint erstmalig modernste Laserdiodentechnologie und Ultrakurzpuls-Festkörperoszillatoren, basierend auf $\mathrm{Cr}^{2+}: \mathrm{ZnS} / \mathrm{ZnSe}$, eindrucksvoll miteinander. Die im Rahmen dieser Arbeit entwickelten neuartigen Lasersysteme zeigen einerseits, wie durch die Erzeugung kohärenter Strahlung der Bereich zwischen $2 \mu \mathrm{m}$ und $3 \mu \mathrm{m}$ abgedeckt werden kann. Andererseits wird durch dieses Konzept eine gleichzeitige Reduktion in Größe und Komplexität erreicht, sowie eine verbesserte Gesamteffizienz und insbesondere Rauschverhalten. All das macht den Ansatz wegweisend für zukünftige Anwendungen im mittelinfraroten Spektralbereich.

Im Rahmen der Dissertation wurden drei einzigartige Lasersysteme entwickelt, wobei jedes davon die Grenzen direkt Dioden-gepumpter Lasertechnologie sprengt. Das erste System wird von einer Einzel-Emitter-Diode aus Indiumphosphid gepumpt und liefert bei einer Impulsdauer von nur 45 fs eine Ausgangsleistung von mehr als $500 \mathrm{~mW}$. Insbesondere stellt dies die erstmalige Umsetzung eines Kerr-Linsen modengekoppelten (KLM) $\mathrm{Cr}^{2+}: \mathrm{ZnS} / \mathrm{ZnSe}$ Oszillators dar, der direkt von einer Diode optisch gepumpt wird. Neben einer außerordentlichen Langzeitstabilität ist das Lasersystem durch ein exzellentes Rauschverhalten charakterisiert. Dies zeigt sich in Messungen des Amplitudenrauschens im modengekoppelten Betrieb. 
Um die Leistungsmerkmale eines typischen Faserlaser-gepumpten $\mathrm{Cr}^{2+}: \mathrm{ZnS} / \mathrm{ZnSe}$ Systems zu erreichen und zu übertreffen, und damit auch die Effizienz einer dem Lasersystem nachgelagerten Anwendung zu steigern, werden zwei Einzel-EmitterDioden in einem Lasersystem der zweiten Generation präzise überlagert. Die dadurch realisierbaren Spitzenleistungen sind knapp einen Faktor drei größer als mit nur einer Diode, während die Rauschcharakteristik unverändert niedrig bleibt.

Im Allgemeinen ist ein Lasersystem, welches zur Erzeugung von mittlerer Infrarotstrahlung von bis zu mehreren zehn Mikrometern eingesetzt wird, außerordentlich komplex. Dies liegt in der komplizierten Verkettung von Verstärker-, Pulskompressions- und optisch parametrischen Konversionsstufen dieser Systeme begründet. Wird stattdessen ein leistungsstarker Mittelinfrarot-Ultrakurzpulslaser verwendet, kann nicht nur die Effizienz von nichtlinearen Prozessen signifikant erhöht, sondern unter Umständen sogar eine Verstärkerstufe überflüssig werden. Mit dem im Rahmen der Dissertation entwickelten Lasersystem der dritten Generation kann genau dies erreicht werden. Mit einer Spitzenleistung von $1 \mathrm{MW}$ bei einer Impulsdauer von nur 28 fs wird die direkte Erzeugung phasenstabiler Mittelinfrarotimpulse in einem nichtlinear optischen Kristall (ZGP) möglich. Dieser nichtlineare Konversionsprozess erzeugt ein mehrere Oktaven breites Spektrum zwischen $4.5 \mathrm{\mu m}$ und $14 \mu \mathrm{m}$ bei Durchschnittsleistungen von mehr als $20 \mathrm{~mW}$.

Die erfolgreiche Entwicklung und Realisierung von drei neuartigen und leistungsstarken direkt Dioden-gepumpten Lasersystemen im mittelinfraroten Spektralbereich legt die Grundlage für eine neue Generation von MIR-Ultrakurzpulslasern. Diese ermöglichen die Durchführung spektroskopischer Messungen mit einer beispiellosen Effizienz und einer Empfindlichkeit am Quantenrauschen. Zugleich ebnen sie den Weg für eine einfacher zugänglichere Alternative zu Synchrotron-ähnlicher Infrarotstrahlung. 


\begin{abstract}
The mid-infrared (MIR) spectral range with wavelengths between $2 \mu \mathrm{m}$ and $20 \mu \mathrm{m}$ holds tremendous potential for the study of complex biological systems, given the abundance of intense and unique molecular absorption lines that can be detected. Consequently, spectroscopic applications of mid-infrared radiation have garnered enormous attention in recent years. A particularly striking example is the combination of multi-MHz-repetition-rate, few-cycle MIR light sources with electric-field-resolved techniques, enabling the recording of amplitude- and phase-resolved molecular signals with unparalleled specificity and shot noise limited sensitivity. Despite the ever-growing research demand, their widespread use is severely hampered by the lack of low-noise, compact, and ultrafast laser systems.
\end{abstract}

In this dissertation, a new generation of table-top mid-infrared laser sources is presented, bringing cutting-edge laser diode technology and few-cycle $\mathrm{Cr}^{2+}: \mathrm{ZnS} / \mathrm{ZnSe}$ solid-state oscillators together for the first time. Not only have these laser systems proven to reliably provide coherent radiation in the $2 \mu \mathrm{m}$ to $3 \mu \mathrm{m}$ region, the simultaneous reduction in size and complexity, accompanied by an improved overall efficiency and - most importantly - noise performance, renders this approach as pioneering for future MIR applications.

In total, three laser systems are developed, each of them pushing the frontiers of directly diode-pumped laser technology. The first one is driven by a single-emitter indium phosphide laser diode and delivers more than $500 \mathrm{~mW}$ of output power combined with pulse durations as short as $45 \mathrm{fs}$. With this first ever directly diodepumped Kerr-lens mode-locked (KLM) $\mathrm{Cr}^{2+}: \mathrm{ZnS} / \mathrm{ZnSe}$ oscillator, experimental results confirm a highly stable operation. In addition, amplitude noise measurements reveal an excellent low-noise performance of the mode-locked laser output.

Driven by the desire to match and exceed the performance of more mature fiber-laser-pumped counterparts and also to boost the efficiency of the envisaged downstream applications, the output of two single-emitter pump laser diodes is carefully combined and implemented into a second-generation design. The achieved peak powers are almost three-times higher compared to before, while the low-noise performance of the KLM output is maintained. 
Typically, the design architecture of laser systems used for generating mid-infrared radiation up to several tens of microns includes a sophisticated chain of amplification, pulse compression and parametric conversion stages. Using a powerful few-cycle mid-infrared oscillator as driving laser source instead not only significantly improves the effectiveness of these nonlinear schemes, but could even supersede the need for initial amplification. The presented third-generation system brings the directly diode-pumped $\mathrm{Cr}^{2+}: \mathrm{ZnS} / \mathrm{ZnSe}$ solid-state laser technology to a new level; with output peak powers reaching $1 \mathrm{MW}$ and pulse durations as short as $28 \mathrm{fs}$, direct generation of CEP-stable mid-infrared pulses in a nonlinear optical crystal (ZGP) becomes feasible and results in a multi-octave-spanning spectrum between $4.5 \mu \mathrm{m}$ and $14 \mu \mathrm{m}$ at more than $20 \mathrm{~mW}$ of average power.

The successful development and realization of the three novel and powerful directly diode-pumped mid-infrared laser systems serves as a foundation for a new generation of few-cycle MIR light sources, capable of performing spectroscopic measurements at unprecedented efficiency and shot noise limited sensitivity, while paving the way towards a more accessible alternative to synchrotron-like infrared radiation. 


\section{Contents}

List of Figures $\quad \mathrm{XV}$

List of Tables $\quad$ xix

List of Acronyms xxi

1 MOTIVATION 1

1.1 MIRanda - A New Ultrafast Workhorse for Mid-Infrared Applications 1

1.2 The Power of Directly Diode-Pumped, Few-Cycle Mid-Infrared Lasers 5

1.3 Thesis Outline . . . . . . . . . . . . . . . . . . 9 9

2 FUNDAMENTALS of Ultrashort Pulse Generation 11

2.1 Formation of Laser Pulses . . . . . . . . . . . . . . . . . . . . 11

2.1.1 Locking the Longitudinal Modes of a Laser Cavity . . . . . . 13

2.1.2 Experimental Realization Methods . . . . . . . . . . 15

2.2 Soliton Pulse Propagation . . . . . . . . . . . . . . . . 20

2.2.1 Dispersion . . . . . . . . . . . . . . . . . . . . . 20

2.2.2 Self-Phase Modulation . . . . . . . . . . . . . . 22

2.2.3 The Fundamental Soliton . . . . . . . . . . . . . . . . 24

2.3 Soliton Mode-Locking . . . . . . . . . . . . . . . . . 25

2.3.1 Gain, Loss, and the Spectral Filtering . . . . . . . . . . . 26

2.3.2 Self-Amplitude Modulation . . . . . . . . . . . . . 27

2.3.3 The Master Equation of Mode-Locking . . . . . . . . . . . . 28

2.3.4 The Effect of Different Dispersion Regimes . . . . . . . . . . 29

2.4 Chapter Summary . . . . . . . . . . . . . . . . . . . 30

3 THE FIRST Directly Diode-Pumped Few-Cycle Cr-Doped II-VI Laser 31

3.1 Chromium-doped II-VI - The Titanium Sapphire of the Mid-Infrared 31

3.2 Challenges of Diode-Pumping . . . . . . . . . . . . . . 34

3.2.1 The Right Choice of Laser Diode Type . . . . . . . . . . . . 35

3.2.2 Properties of Single-Emitter Diodes . . . . . . . . . . . . . 36

3.2.3 Effects on the Laser System and the Few-Cycle Pulse Generation 40

3.2.3.1 Mode-Matching Considerations . . . . . . . . . 40

3.2.3.2 Technical Challenges . . . . . . . . . . . . 42

3.3 Few-Cycle Pulse Generation via Kerr-Lens Mode-Locking . . . . . . 44 
3.3.1 Pump Setup and Resonator Design . . . . . . . . . . . . . . 44

3.3.2 The Importance of Intra-cavity Dispersion Management . . . 48

3.3.3 Characterization of the Mode-Locked Output . . . . . . . . 52

3.3.4 The Success of Powerful Diode-Pumped Operation . . . . . 61

3.4 Chapter Summary . . . . . . . . . . . . . . . . . . 64

4 REACHING THE PERFORMANCE of the State of the Art 67

4.1 Challenges of Reaching Higher Average and Peak Powers . . . . . . 68

4.1.1 Pump Setup Considerations . . . . . . . . . . . . . . . . 69

4.1.2 Effects on the Mode-Locking Performance . . . . . . . . . . 70

4.2 Comparison and Improvements over MIRanda1 . . . . . . . . . . . 77

4.2.1 Pump and Cavity Design . . . . . . . . . . . . . 77

4.2.2 Mode-Locked Performance . . . . . . . . . . . . . . . . 80

4.3 Chapter Summary . . . . . . . . . . . . . . . . . . . 88

5 PUSHING THE PEAK POWER for Direct Mid-Infrared Generation 91

5.1 Challenges of Reaching Higher Peak Powers . . . . . . . . . . . . . 95

5.2 Experimental Achievements . . . . . . . . . . . . . . . . 98

5.2.1 Pump and Oscillator Setup . . . . . . . . . . . . 99

5.2.1.1 Intra-Cavity 4f-Telescopes . . . . . . . . . . . . . 101

5.2.1.2 Selection of the Curved Mirror Pair . . . . . . . . . 103

5.2.2 Mode-Locked Performance and the Effect of Air Dispersion . 104

5.2.3 Towards Even Higher Peak Power Levels . . . . . . . . . . . 113

5.3 Frequency Down-Conversion into the Mid-Infrared . . . . . . . . . . 117

5.3.1 Intra-Pulse Difference Frequency Generation in a ZGP Crystal117

5.3.2 Characterization of the Generated Radiation . . . . . . . . . 119

5.4 Chapter Summary . . . . . . . . . . . . . . . . . . . . . . 124

6 CONCLUSION and OUTLOOK 127

6.1 Summary . . . . . . . . . . . . . . . . . . 127

6.2 A New Ultrafast Workhorse for Spectroscopic Applications? . . . . 129

6.3 Closing Remarks . . . . . . . . . . . . . . . . . . . . . . 132

$\begin{array}{lr}\text { A APPENDIX } & \mathbf{1 3 5}\end{array}$

A.1 List of Publications . . . . . . . . . . . . . . . . . . 135

A.2 Data Archiving . . . . . . . . . . . . . . . . . 137

$\begin{array}{ll}\text { References } & 139\end{array}$ 


\section{List OF Figures}

1.1 Absorption spectra of athmospheric (gaseous) molecules and human blood serum in the MIR region. . . . . . . . . . . . . . . .

1.2 Comparison of the typical scheme used for mid-infrared generation and its vast simplification offered by MIRanda . . . . . . . . . 6

1.3 Simulated phase-matching curves for the generated signal (idler) wavelengths in LGS and GaSe via frequency down-conversion . . . .

2.1 Illustration of a two-mirror cavity and a 4-level gain medium along with the supported longitudinal and transverse mode distributions .

2.2 Simulated temporal behaviour of the squared amplitude of the total electric field for random and constant phases. . . . . . . . . . . . . 14

2.3 Modeling of the intra-cavity beam radius for the case of zero and high peak power to illustrate hard-aperture KLM . . . . . . . . . 17

2.4 Modeling of the intra-cavity beam radius for the case of zero and high peak power to illustrate soft-aperture KLM . . . . . . . . . 18

2.5 Two-dimensional simulation of the Kerr strength parameter for varying crystal positions and distances between the focusing lenses . . .

2.6 Simulated spectrograms to illustrate the effect of SPM and negative GDD on the temporal and spectral pulse shape . . . . . . . . . 23

2.7 Propagation of a fundamental soliton . . . . . . . . . . . . 25

2.8 Principal factors influencing the generation and evolution of ultrashort pulses ............................ 25

2.9 Qualitative behaviour of the SAM functions $\mathfrak{F}\left(|A(z, \tau)|^{2}\right)$ for hardand soft-aperture KLM . . . . . . . . . . . . . . 27

2.10 Effect of dispersion and SPM on the chirp, pulse width and stability 28

3.1 Absorption and emission spectra of Ti:sapphire and Cr:ZnS/ZnSe crystals and coordinational diagram of the laser transitions . . . . .

3.2 Illustration of the output emission of a (wide stripe) laser diode and the small size and components of a C-Mount diode . . . . . . . .

3.3 Beam quality and beam profile measurements of the laser diode output for different driving currents . . . . . . . . . . . . . .

3.4 Qualitative comparison of the amplitude noise of available pump sources for $\mathrm{Cr}: \mathrm{ZnS} / \mathrm{ZnSe}$ oscillators . . . . . . . . . . . .

3.5 Simulation of the overlapped volume between the laser diode beam and the fundamental cavity beam along the pump absorption length 
3.6 Pictures of the laser diode and crystal mount and schematic sketch of a classic and folded bow-tie cavity . . . . . . . . . . . . . . . 42

3.7 Laser diode output power characteristics and beam quality . . . . . 45

3.8 Simulated pump beam propagation for the fast and slow axis and measured change in pump spot diameters for increasing current levels 45

3.9 Schematic of the experimental setup of MIRanda1 and a picture of the real laser system . . . . . . . . . . . . . . 46

3.10 Illustration of the difference in beam radii for AR-coated and Brewstercut crystals . . . . . . . . . . . . . . . .

3.11 Theoretical second-order dispersion and measured reflectivity curves for incorporated intra-cavity elements . . . . . . . . . . . . . . 49

3.12 Calculated stability zones and beam radii (CW/ML) for MIRanda1 50

3.13 Illustration of the intra-cavity dispersion tuning and its effect on the mode-locked spectral shape and FWHM . . . . . . . . . . 51

3.14 SHG-FROG measurement of the mode-locked laser output . . . . . 53

3.15 CW slope efficiency for varying laser diode pump currents . . . . . 54

3.16 Measured output characteristics and slope efficiencies of MIRanda1 55

3.17 Amplitude noise measurement setup and RF spectrum of the carrier

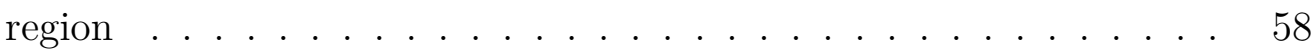

3.18 Amplitude noise measurements and integrated RMS noise for the frequency-doubled output of MIRanda1 . . . . . . . . . . 60

3.19 Potential explanations for the powerful diode-pumped operation . . 62

4.1 Simulated pulse propagation in a ZBLAN fiber illustrating the effect of input power and pulse duration on Raman-induced soliton selffrequency shifting . . . . . . . . . . . . . . .

4.2 Two ways to increase the effective pump power in a $\mathrm{Cr}: \mathrm{ZnS} / \mathrm{ZnSe}$ system via two single-emitter laser diodes . . . . . . . . . . . .

4.3 Soliton mode-locking in the temporal and spectral domain accompanied by the appearance of a background radiation. . . . . . . . . . .

4.4 Recorded mode-locking instabilities in the form of a continuous-wave background and multi-pulsing . . . . . . . . . . . . . 72

4.5 Representative model for the nonlinear ABCD-matrix calculations . 74

4.6 Two-dimensional representation of the Kerr strength for varying crystal and curved mirror displacements . . . . . . . . . . . 75

4.7 Kerr strength simulations for varying arm length ratios . . . . . . . 76

4.8 Performance of the new diode mount and schematic of the two pump collimation assemblies . . . . . . . . . . . . . . 78

4.9 Experimental setup of MIRanda2 with a picture of the laser system 79

4.10 Increase of the output coupling ratio in mode-locked operation . . . 81

4.11 Stability zone scan and KLM performance at varying arm ratios . . 82 
4.12 Calculated round-trip dispersion of MIRanda2 and theoretical reflectivity and dispersion curves for the two sets of highly reflective mirrors . . . . . . . . . . . . . . . . .

4.13 Observed jump in the ML output polarization when measured at constant cavity parameters . . . . . . . . . . . .

4.14 SHG-FROG measurement of the output pulses at $25 \%$ and $3 \%$ output coupling rate ....................... 84

4.15 Output performance of MIRanda2 . . . . . . . . . . . . . 86

4.16 Amplitude noise measurements and integrated RMS noise of the mode-locked output of MIRanda2 . . . . . . . . . . . . .

5.1 Comparison of inter-pulse and intra-pulse difference frequency generation schemes . . . . . . . . . . . . . . . . .

5.2 Illustration of enhanced mid-infrared generation via shorter driving pulse durations and a lower repetition rate . . . . . . . . . . . . . 94

5.3 Calculation of the single-pass B-integral inside the Cr:ZnS crystal . 96

5.4 Part of the laser pulse spectrum after linear propagation and perturbed soliton propagation . . . . . . . . . . . . . 98

5.5 Schematic of the two-lens pump focusing unit . . . . . . . . . . . 99

5.6 Experimental layout of MIRanda3 . . . . . . . . . . . . . . . . . . 100

5.7 Pictures of the actual laser assembly . . . . . . . . . . . . . . 101

5.8 Continuous-wave output beam corresponding to different cavity arm lengths . . . . . . . . . . . . . . . . . . . 102

5.9 Mode-locked output beam profile for the $45 \mathrm{MHz}$ cavity . . . . . . . 103

5.10 Two-dimensional simulations of the Kerr strength parameter for different radii of curvature (ROC) of the spherical cavity mirrors . . 104

5.11 Stability zone scan and calculated beam radii along the cavity axis . 105

5.12 Theoretical dispersion values and measured reflectivity curves of intra-cavity elements . . . . . . . . . . . . . . . 106

5.13 Illustration of the intra-cavity dispersion tuning and the effect on the mode-locked spectral width along with the $\nu_{\text {rep }}=23 \mathrm{MHz}$ output pulse train . . . . . . . . . . . . . . . . . 106

5.14 Characterization of the generated laser pulse durations of MIRanda3 via SHG-FROG measurements . . . . . . . . . . . . . . . . 107

5.15 Change of the spectral shape and pulse duration upon purging with dry nitrogen . . . . . . . . . . . . . . . . . . 109

5.16 Change of the spectral shape and pulse duration upon purging at slightly increased net negative intra-cavity dispersion . . . . . . . . 110

5.17 Output characteristics of MIRanda3 . . . . . . . . . . . . . . 111

5.18 Amplitude noise measurements and integrated RMS noise of the mode-locked output of MIRanda3 . . . . . . . . . . . . . 
5.19 Performance of the newly designed dispersive mirror pairs for the 1.8-3.2 um spectral range . . . . . . . . . . . . . . . . . . . . 114

5.20 Sketch and picture of the additional telescope added to the laser cavity of MIRanda3 for test purposes . . . . . . . . . . . . . . 115

5.21 Optical absorption of ZGP and simulated type I phase-matching (PM) curves for three different pump wavelengths . . . . . . . . . 118

5.22 Mid-infrared generation setup along with the measured power levels and beam profiles behind the ZGP crystal . . . . . . . . . . . 120

5.23 Generated (mid-)infrared spectra with a ZGP crystal for the two observed maxima . . . . . . . . . . . . . . . . . . 122

5.24 Calculation of the single-pass B-integral inside the ZGP crystal . . . 124

6.1 3D mechanical design of the new laser diode collimation unit . . . . 131

6.2 3D mechanical design of the laser prototype and its experimental realization . . . . . . . . . . . . . . . . . 132 


\section{LIST OF TABLES}

2.1 Important second- and third-order nonlinear optical susceptibilities and corresponding nonlinear processes . . . . . . . . . . 17

2.2 Linear and nonlinear refractive index of selected materials and their group velocity dispersion . . . . . . . . . . . . . . 21

3.1 Material and spectroscopic characteristics of the broadband laser materials $\mathrm{Cr}^{2+}: \mathrm{ZnS} / \mathrm{ZnSe}$ and $\mathrm{Ti}^{3+}$ :sapphire. . . . . . . . . 33

3.2 Desired output characteristics of a laser diode to be used for the few-cycle oscillators presented in this thesis . . . . . . . . . 36

3.3 Best simulation results for given $M^{2}$-values of the pump beam and focus size $\mathrm{w}_{0, f}$ for the fast axis . . . . . . . . . . .

3.4 Comparison of the output characteristics of the previous record versus the first demonstration of diode-pumped few-cycle $\mathrm{Cr}^{2+}$-doped II-VI oscillators ........................ 65

4.1 Simulation parameters ... . . . . . . . . . . . . . 74

4.2 Comparison of the laser performance and the respective improvements achieved over the first directly diode-pumped $\mathrm{Cr}^{2+}$-doped II-VI laser system . . . . . . . . . . . . . . . .

5.1 Dependance of the shortest mid-IR wavelengths reachable for different driving pulse durations . . . . . . . . . . . . . . . . . . 94

5.2 Measured focal spot diameters and distance for a given set of optics 99

5.3 Average power levels measured directly behind different long-pass filters for the 1 st and 2 nd maximum . . . . . . . . . . . . . . . 123

6.1 Comparison of the laser performance of the three directly diodepumped $\mathrm{Cr}^{2+}$-doped II-VI laser systems presented in this thesis . . 



\section{List OF ACRONYMS}

$\begin{array}{ll}\text { AM } & \text { Amplitude modulation } \\ \text { AOI } & \text { Angle of incidence } \\ \text { AR } & \text { Anti-reflective } \\ & \\ \mathrm{BaF}_{2} & \text { Barium fluoride } \\ \text { BBO } & \text { Beta barium borate, } \beta \text {-BaB } \mathrm{O}_{4} \\ & \\ \text { CEO } & \text { Carrier-envelope offset } \\ \text { CEP } & \text { Carrier-envelope phase } \\ \text { CM } & \text { Chirped mirror } \\ \text { Cr:ZnS } & \text { Chromium-doped zinc sulfide, } \mathrm{Cr}^{2+}: \mathrm{ZnS} \\ \text { Cr:ZnSe } & \text { Chromium-doped zinc selenide, } \mathrm{Cr}^{2+}: \mathrm{ZnSe} \\ \mathrm{CSP} & \text { Chirped solitary pulse } \\ \text { CW } & \text { Continuous-wave } \\ & \\ \text { DC } & \text { Direct current } \\ \text { DFG } & \text { Difference frequency generation } \\ \text { DM } & \text { Dispersive mirror } \\ & \\ \text { EOS } & \text { Electro-optical sampling } \\ \text { Er } & \text { Erbium } \\ & \\ \text { FM } & \text { Frequency modulation } \\ \text { FOM } & \text { Figure of merit } \\ \text { FROG } & \text { Frequency-resolved optical gating } \\ \text { FRS } & \text { Field-resolved spectroscopy } \\ \text { FS } & \text { Fused silica } \\ \text { FTIR } & \text { Fourier-transform infrared } \\ \text { FTL } & \text { Fourier-transform limit } \\ \text { FWHM } & \text { Full width at half maximum } \\ \text { GaSe } & \text { Gallium selenide } \\ & \\ & \end{array}$


GD Group delay

GDD Group delay dispersion

GVD Group velocity dispersion

HITRAN High-resolution transmission molecular absorption database

HR Highly-reflective

IDFG Intra-pulse difference frequency generation

InGaAs Indium gallium arsenide

InP Indium phosphide

IR Infrared

ISO International organization for standardization

KLM Kerr-lens mode-locking/mode-locked

LGS Lanthanum gallium silicate

$\mathrm{LiIO}_{3} \quad$ Lithium iodate

$\mathrm{MgF}_{2} \quad$ Magnesium fluoride

MIR Mid-infrared

ML Mode-locking

MOPA Master oscillator power amplifier

ND Neutral density

NIR Near-infrared

OC Output couler/coupling

OPA Optical parametric amplifier/amplification

OPO Optical parametric oscillator/oscillation

PBC Polarizing beam combiner

$\mathrm{PbS} \quad$ Lead sulfide

PD Photodetector

pf-SNOM Peak force scattering-type scanning near-field optical microscopy

PM Phase-matching 
PPLN Periodically poled lithium niobate

QCL Quantum cascade semiconductor laser

RBW Resolution bandwidth

RF Radio-frequency

RH Relative humidity

RIN Relative intensity noise

RMS Root mean square

RO Relaxation oscillation

ROC Radius of curvature

RQPM Random quasi-phase-matching

s-SNOM Scattering-type scanning near-field optical microscopy

SAM Self-amplitude modulation

SESAM Semiconductor saturable absorber mirror

SH Second harmonic

SHG Second-harmonic generation

SNOM Scanning near-field optical microscopy

SNR Signal-to-noise ratio

SPM Self-phase modulation

TE Transverse electromagnetic

TEC Thermoelectric cooling

TEM Transverse electromagnetic mode

Ti:Sa Titanium-doped sapphire, Ti: $\mathrm{Al}_{2} \mathrm{O}_{3}$

Tm Thulium

TM Transition metal

TOD Third-order dispersion

$\mathrm{U}: \mathrm{CaF} \quad$ Uranium-doped calcium fluoride, $\mathrm{U}^{3+}: \mathrm{CaF}_{2}$

UV Ultraviolet

VA Voltage amplifier

XFROG Cross-correlation frequency-resolved optical gating 
YAG Yttrium aluminium garnet

ZBLAN $\mathrm{ZrF}_{4}-\mathrm{BaF}_{2}-\mathrm{LaF}_{3}-\mathrm{AlF}_{3}-\mathrm{NaF}$

ZGP Zinc Germanium Phosphide, $\mathrm{ZnGeP}_{2}$

ZnS Zinc sulfide

ZnSe Zinc selenide 


\section{MOTIVATION}

\subsection{MIRanda - A New Ultrafast Workhorse for Mid-Infrared Applications}

\section{The advent of mid-infrared laser sources and spectroscopy}

The second half of the last century was marked by profound technological developments and most of them are now taken for granted in daily life. The foundations for one of the most outstanding inventions have been layed in 1917, when Albert Einstein predicted the phenomenon of "stimulated emission" [1] — a fundamental concept that became the backbone of laser physics. However, it took more than 40 years before the first working prototype of a laser was finally demonstrated by Theodore Maiman in 1960 [2], putting lasers onto a successful path that persists until today.

Inspired by the first discovery of laser action in a ruby crystal, an intense search for other suitable material was triggered, and Peter Sorokin and Mirek Stevenson were the first to demonstrate a 4-level solid-state laser based on uranium-doped calcium fluoride $\left(\mathrm{U}^{3+}: \mathrm{CaF}_{2}\right)[3]$. Notably, this world's second laser was emitting infrared light at a wavelength of $2.49 \mu \mathrm{m}$, unlike the clearly visible red light generated by a ruby laser $(0.694 \mu \mathrm{m})$. Even though it never proved practical, it opened the door to an enormously promising region for spectroscopic applications - the mid-infrared (MIR).

Often referred to as the molecular "finger-print" region $[4,5]$, the MIR range between $2.5 \mu \mathrm{m}$ and $20 \mu \mathrm{m}\left(500-4000 \mathrm{~cm}^{-1}\right)$ is home to a grand variety of intense molecular absorption lines of organic and inorganic substances [6] (see figures 1.1). Owing to the energy associated with infrared radiation, which is too low to excite electrons to higher-lying energy levels, electromagnetic radiation can resonate with rotational and/or vibrational modes of chemical bonds or functional groups within the molecule, such that information on the chemical arrangement as well as the intramolecular interactions can be retrieved [7]. Probing these fundamental modes directly in the mid-infrared is a great advantage over near-infrared (NIR) spectroscopy, where molecular absorption is due to anharmonic overtone and combination bands, making NIR spectra very complex and more difficult to interpret [8]. 
(a)

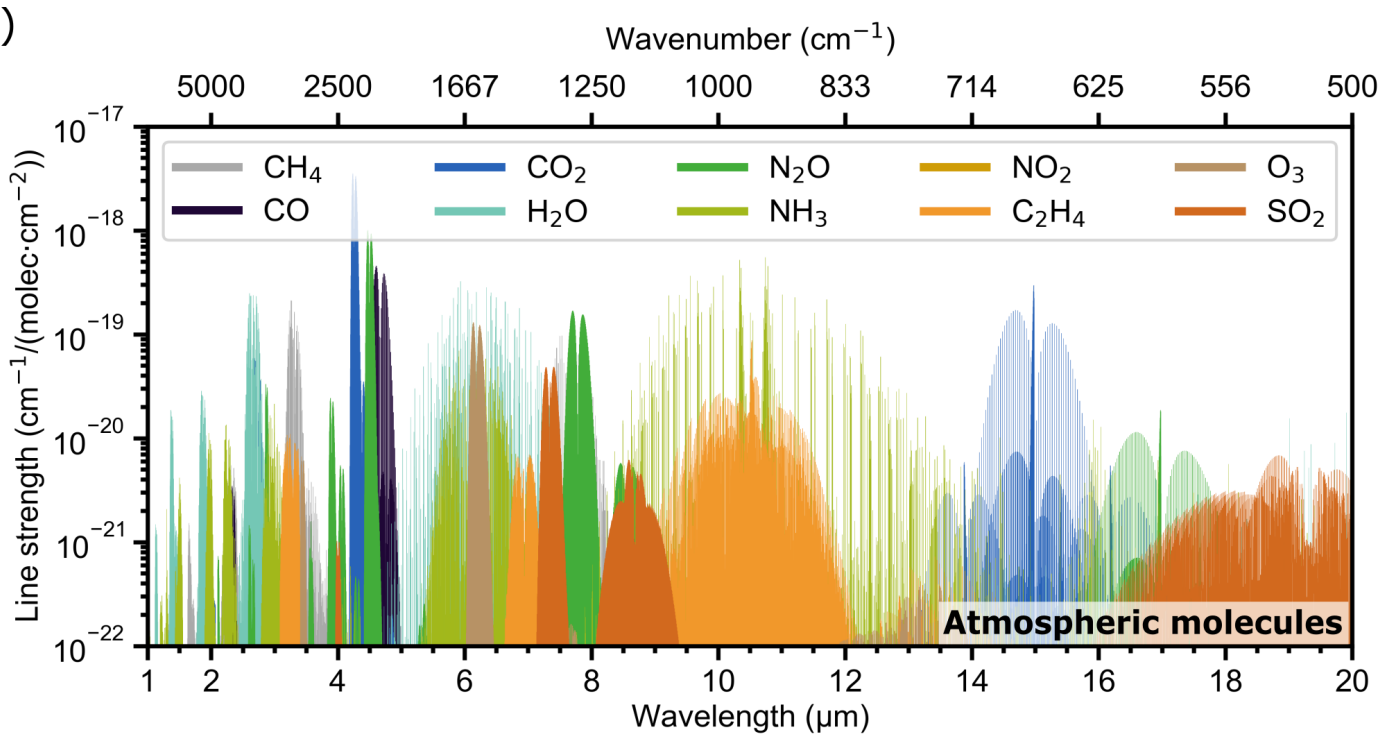

(b)

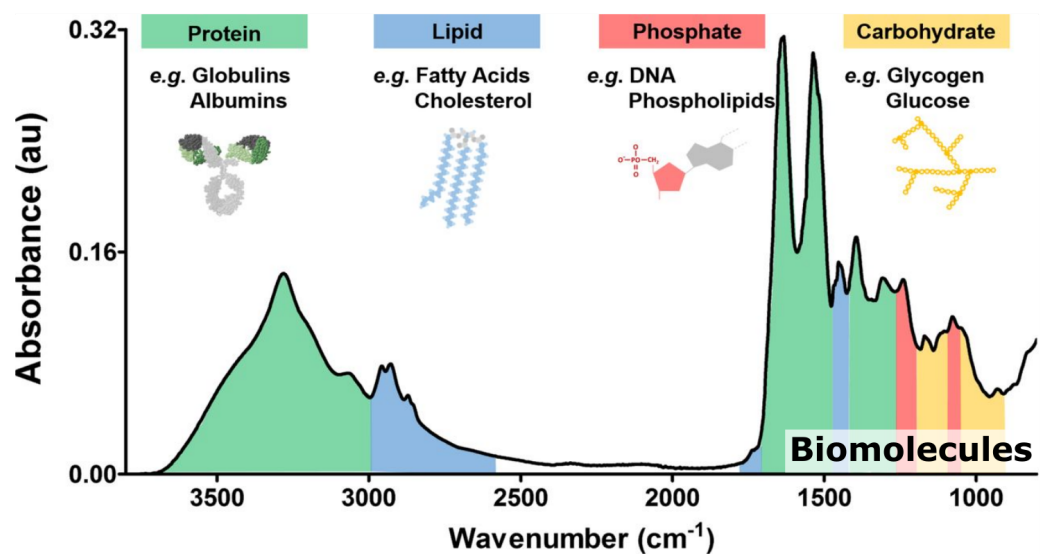

Fig. 1.1: Absorption spectra of athmospheric (gaseous) molecules and human blood serum in the MIR region. (a) Narrow-band absorption lines of typical atmospheric molecules in the range between $1 \mu \mathrm{m}$ and $20 \mu \mathrm{m}$. The data were obtained from HITRAN simulations. (b) Absorption spectrum of human blood serum and identifiable biomolecules analyzed with FTIR spectroscopy [9]. Compared to measurements in the gas-phase (see (a)), where the molecules are isolated and do not interact with each other, the individual absorption features in the liquid phase start to broaden and smear out, eventually resulting in a continuous appearance of the absorbance.

Spectroscopic measurements aiming to address as many different molecules as possible benefit greatly from broadband MIR radiation such as those emitted from synchrotrons [10-12] or globars. The latter is a thermal source, which emits blackbody radiation from an electrically heated material. Even though this method is readily available and straightforward to use, with several watts of average power being generated, thermal radiation lacks both spatial and temporal coherence. A 


\subsection{MIRanda - A New Ultrafast Workhorse for Mid-Infrared Applications 3}

high degree of coherence, however, is an important prerequisite for a broad range of mid-infrared applications, including the emerging fields of of nano-imaging [13-15] as well as time-domain $[14,16]$ and frequency comb spectroscopy [17-19]. Therefore, synchrotrons are often preferred over globars because their higher brilliance and thus highly spatially confined photon flux allows for an improved signal-to-noise ratio [12] - especially important for measurements requiring good spatial resolution. Synchrotron radiation is generated by electrons that are accelerated and stored in large ring structures. These electrons travel in bunches of about $1 \mathrm{~mm}$ to $10 \mathrm{~mm}$ length, and produce short pulses of light when deflected by magnetic fields. With emitted wavelengths continuously extending from the very far-infrared to the highenergy X-ray regime, the light is confined to temporal widths of tens of picoseconds to a few nanoseconds (depending on the ring and its respective mode of operation) [12]. Consequently, phenomena can also be investigated in a time-resolved manner $[20,21]$, but with restricted access to nonlinear interactions happening on shorter time scales [22]. Other serious drawbacks of synchrotrons arise from the very limited beam time, the extremely high costs associated with their elaborate infrastructure as well as their MIR radiation power being limited to a few milliwatts only [23]. In this respect, broadband coherent MIR systems based on laser technology are a highly sought after alternative.

The search for suitable mid-infrared laser sources is, unfortunately, not straightforward. To address various molecular species simultaneously, MIR generation systems need to provide direct lasing in the $2.5 \mu \mathrm{m}$ to $20 \mu \mathrm{m}$ range, while covering a broad portion of the mid-IR at once or in a broadly tunable manner. Among others, quantum cascade semiconductor lasers (QCLs) are used for highly accurate and quantitative measurements, given their very narrow emission linewidths $[24,25]$. Though they can be made quite compact in design, the limited wavelength tunability impedes parallel sensing of multiple molecular species, and several QCLs would need to be used together. Another promising approach is to exploit nonlinear frequency down-conversion into the MIR using mature near-infrared laser sources. Thus, optical parametric oscillators (OPOs) and amplifiers (OPAs) [26-30] have become very popular in recent years. Pulse energies reaching up to at least the $\mu \mathrm{J}$-level combined with an extremely large tunability allow to cover spectral domains, where laser options are typically scarce or require cryogenic cooling of the gain material. However, their sensitivity to misalignment [31] and crystal orientation due to phase-matching and energy conservation requirements increases the overall complexity. By contrast, a relatively simple method is based on collinear difference frequency generation (DFG), and is known as intra-pulse DFG (IDFG) [32-37]. Difference frequencies are generated when different frequency components of a single ultrabroadband laser pulse are mixed together, resulting in an intrinsically carrier-envelope phase (CEP)-stabilized signal regardless of the CEP-stability of the incident pulse. In recent years, the technique of IDFG was shown to result in 
the generation of several tens of milliwatts of MIR power with super-octave spectral coverage [32-37], providing ideal prerequisites for spectroscopic measurements. Moreover, due to the intrinsic CEP-stability of the mid-infrared radiation [38], new spectroscopic techniques can be exploited [39, 40].

\section{About ultrafast lasers and their impact on MIR spectroscopy}

Coherent light sources have enabled methods such as Fourier-transform infrared (FTIR) spectroscopy [6] to extensively advance the progress in wide-ranging fields from atmospheric and trace-gas analysis [41] to biology and medicine [42-44]. Compared to measurements performed with conventional dispersive spectrometers, FTIR-based techniques are superior due to the higher spectral accuracy, better signal-to-noise ratio (SNR) and sensitivity, as well as optical throughput $[45,46]$. Moreover, switching from continuous-wave (CW) to pulsed laser operation comes along with further advantages: Firstly, pulsed laser light is much more intense to exploit frequency down-conversion schemes in nonlinear optical crystals [47], such that a much wider portion in the mid-infrared can be covered - often in a single and compact optical arrangement [36]. Secondly, the temporal structure of the pulse - especially when its CEP and repetition rate are well controlled in the form of a frequency comb [48] — opens up new detection schemes like electro-optical sampling (EOS) [39, 49] as well as time-resolved spectroscopic techniques [16, 17, 40, 50]. Among them, field-resolved spectroscopy (FRS) in the molecular fingerprint region relies on EOS to gain direct access to the spectral amplitude and phase of the recorded waveforms. Moreover, it allows temporally separating the vibrational response of a sample from the high-power ultrashort excitation event, making measurements with an unparalleled specificity and sensitivity over the entire molecular fingerprint region feasible.

The potential of lasers for ultrafast spectroscopic applications, however, is strongly correlated with the pulse duration they can deliver [14]. Indeed, ultrashort light pulses with durations of less than a hundred femtoseconds $\left(1 \mathrm{fs}=10^{-15} \mathrm{~s}\right)$ enable scientists to study and control motions on ever shorter timescales and with increasing temporal resolution [51-53], with the shortest pulses reaching down to the attosecond scale [54]. The generation of ultrashort pulses has its origin in 1964, when the principle of mode-locking (ML) was discovered [55, 56]. Since then, new techniques - in particular semiconductor saturable absorber ML [57] and Kerr-lens ML [58] - greatly advanced the frontiers of femtosecond solid-state laser technology [59, 60], and pulse durations down to few optical cycles can now be readily generated [61-64]. 


\section{MIRanda: Advance a Just Cause}

Nowadays, laser systems often involve a chain of complicated setups and many of them are not likely to leave the research laboratories due to their complexity and difficult maintenance. Consequently, laser-based applications will struggle to be widely adopted, thus severely hampering the rate of new scientific discoveries in the emerging field of mid-infrared spectroscopy and microscopy. In this $\mathrm{PhD}$ thesis, MIRanda, a new era of MIR laser systems is presented, generating highly-intense optical few-cycle pulses ${ }^{1}$ at multi- $\mathrm{MHz}$ repetition rates directly in the spectral region between $2 \mu \mathrm{m}$ and $3 \mu \mathrm{m}$. Thanks to the innovative combination of direct diode-pumping with ultrafast Kerr-lens ML Cr:ZnS/ZnSe laser technology, high photon-flux can be generated at rather exotic wavelengths and with intrinsic low amplitude noise performance, vastly enhancing the efficiency, sensitivity, and statistics in spectroscopic experiments. Combined with a highly compact design, it will help to advance next-generation molecular diagnostics performed in-house, aiming at health monitoring and disease detection via laser-based human blood analysis ${ }^{2}$. Moreover, underpinning all these developments can be formulated as a Just Cause $^{3}$ : Developing means to monitor and preserve the health of every human being in the best possible way and to the benefit of everyone.

\subsection{The Power of Directly Diode-Pumped, Few-Cycle Mid-Infrared Lasers}

The study of fundamental molecular vibrations in the range between $2.5 \mu \mathrm{m}$ and $20 \mu \mathrm{m}$ requires laser systems that can provide broadband MIR laser light. Direct generation of coherent emission in the molecular fingerprint region is, however, still rather challenging, even though much work has been undertaken to push the emission of ultrafast solid-state oscillators toward ever longer wavelengths [26, 47, 67, 68].

In the $1.9 \mu \mathrm{m}$ to $3.0 \mu \mathrm{m}$ region, pulsed laser oscillators and amplifier systems based on thulium- $[69,70]$, holmium- $[71,72]$ and chromium- $[33,64,73]$ doped gain media have proven to provide peak power levels sufficient for further nonlinear fre-

\footnotetext{
${ }^{1}$ At a given wavelength, a single-cycle pulse is the shortest possible waveform that occurs when the electric field performs only one oscillation period within the pulse envelope. For a laser with output pulse duration $\tau_{0}$ and central frequency $\nu_{0}$, the number of optical cycles is calculated as: $N=\tau_{0} \cdot \nu_{0}[65]$.

${ }^{2}$ Lasers4Life project: www.lasers4life.de, www.mukkozpont.hu

3 "A Just Cause is a specific vision of a future state that does not yet exist. [...] It describes the world we hope to live in and will commit to help build [...] and the goal is not to win, but to keep playing for advancing something bigger than ourselves." [66]
} 
quency down-conversion schemes into the MIR. Besides fiber and bulk architectures, thin-disk technology has attracted growing interest in these materials, mainly due to its power and energy scalability [74]. Owing to the efficient cooling geometry of the gain medium and the reduced thermal lensing effects, record output powers of more than several watts in mode-locked operation can be achieved $[71,75]$. However, the high intra-cavity powers make these systems prone to detrimentally high heat load and optical component damage, while suffering from long initial pulse durations that are attributed to the limited emission bandwidth of the disk material. Attempts have been made to incorporate broadband gain media such as $\mathrm{Cr}: \mathrm{ZnS} / \mathrm{ZnSe}$ into a thin-disk geometry, but it is impeded by the huge thermal load associated to their intrinsically large quantum defect [76]. Though thermal lensing could be reduced in a highly elaborate spinning ring gain element approach [77], its applicability to a mode-locked oscillator is still uncertain.

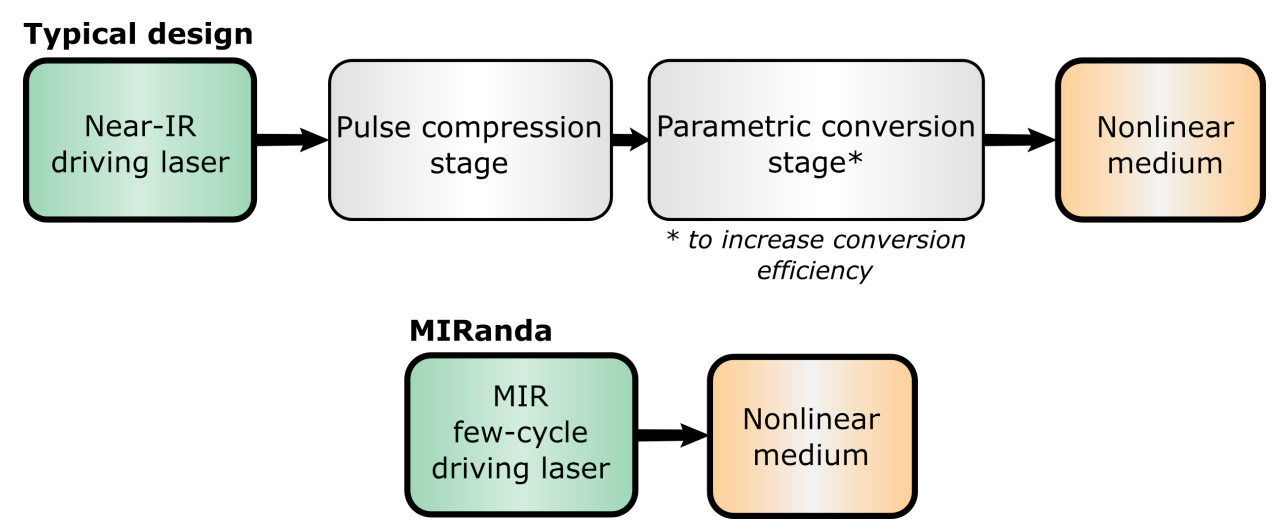

Fig. 1.2: Comparison of the typical scheme used for mid-infrared generation and its vast simplification offered by MIRanda.

To access the longer wavelengths, most frequency down-conversion schemes thus rely on more mature and powerful near-infrared driving lasers, albeit with the need for pulse compression or even parameteric conversion stages to increase pulse energies and improve conversion efficiencies (see figure 1.2). In the following, the advantages of directly diode-pumped as well as few-cycle laser systems with direct emission in the MIR will be presented which — when all characteristics are united - can dramatically simplify the overall scheme.

\section{Direct lasing in the mid-infrared region}

MIR radiation can be generated in a relatively straightforward way by means of nonlinear optical crystals in an IDFG arrangment, and non-oxide materials are preferred 
over oxide-based ones, whose performance is greatly affected by the onset of multiphonon and multi-photon absorption [26]. However, pumping non-oxide crystals with 1-um near-infrared driving systems is extremely difficult and limitations arise from residual absorption loss, unfavourable transparency range or phase-matching (PM), while crystal quality and availability can cause further problems [78]. By contrast, moving to longer pump wavelengths not only results in a smaller quantum defect and a crystal transparency penetrating deeper into the mid-IR, but can also improve the PM bandwidths as well as the overall conversion efficiency [26, 32, 67]. Consequently, direct lasing in the MIR region beyond a wavelength of $2 \mu \mathrm{m}$ is of great interest for applications and few-cycle driving systems like those based on $\mathrm{Cr}^{+2}: \mathrm{ZnS} / \mathrm{ZnSe}$ gain media are highly attractive in this respect. As an example, the phase-matching (PM) curve for frequency down-conversion in a LGS crystal, pumped by a typical 1- $\mu \mathrm{m}$ Yb:YAG laser [79, 80], is compared with that in a GaSe crystal, which can be driven by Cr:ZnS/ZnSe-laser technology [33] (Fig. 1.3). The simulations clearly illustrate the broader span of mid-infrared wavelengths that can be covered via $2 \mu \mathrm{m}$-based driving systems.

(a)

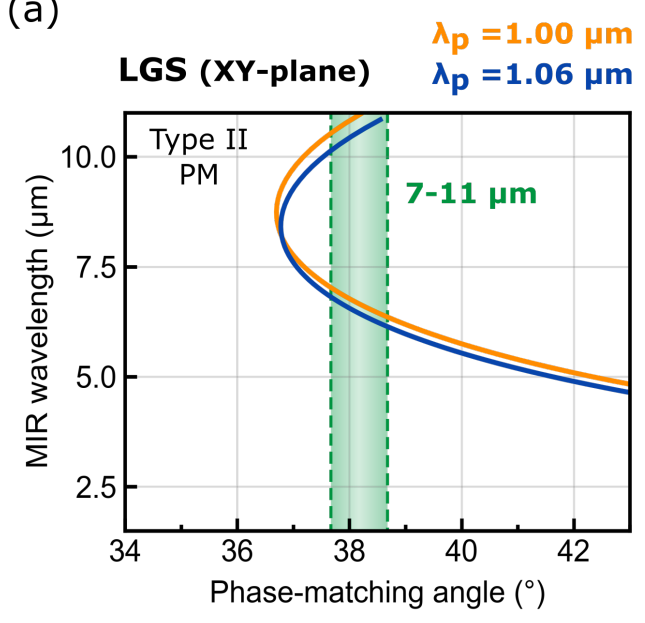

(b)

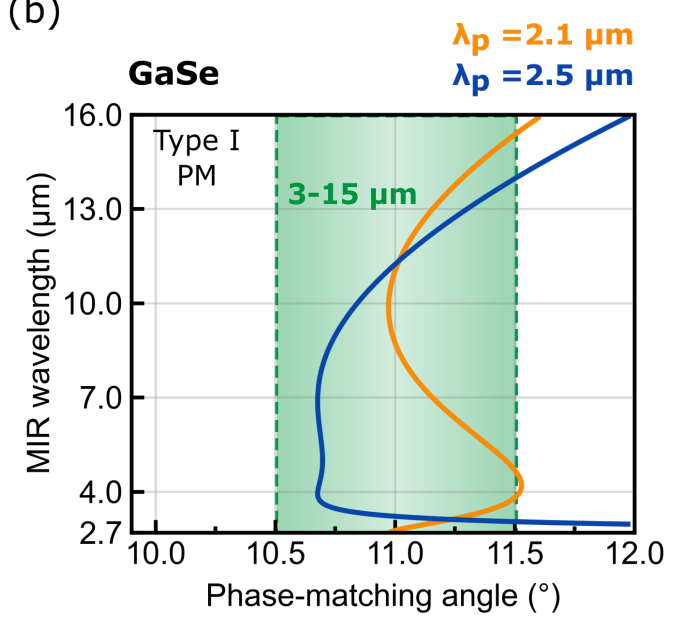

Fig. 1.3: Simulated phase-matching (PM) curves for the generated signal (idler) wavelengths in (a) LGS and (b) GaSe via frequency down-conversion. To resemble an IDFG geometry, where frequency components of a single pump beam mix together and generate mid-IR radiation, two different pump wavelengths $\left(\lambda_{p}\right)$ were assumed. They correspond to the shortest (orange) and longest (blue) wavelength components of an incident pump beam as they are readily emitted from ultrafast Yb:YAG or Cr:ZnS/ZnSe laser systems. For a slight variation in angle of $\pm 0.5^{\circ}$, the achievable MIR wavelengths are shaded in green. They indicate an "ideal" angle of about $38^{\circ}$ for LGS and $11^{\circ}$ for GaSe, which is in good agreement with the PM angles reported for LGS [79, 80] and GaSe [33]. Also, the phase-matching bandwidth offered by a 2 um-pumped GaSe crystal is larger than obtained with a 1 mm-pumped LGS crystal, which allows a broader portion of the MIR to be covered via frequency down-conversion. Note that, compared to GaSe, LGS is biaxial and the propagation is simulated in one of the crystal's principal planes (here: XY). The calculations were performed with the open-source software $S N L O$. 


\section{Direct generation of few-cycle laser pulses}

Since frequency down-conversion techniques like IDFG arise from quadratic $\left[\chi^{(2)}\right.$. type] nonlinear optical effects, the intensity of the driving pump pulse and its associated pulse duration have a strong impact on the conversion efficiency of the process [81]. Using the few-cycle pulses directly available from $\mathrm{Cr}: \mathrm{ZnS} / \mathrm{ZnSe}$ oscillators, as demonstrated in this thesis, will thus provide significant advantage over existing femtosecond pump sources like Er/Tm-fiber lasers [82, 83] and optical parametric generators/amplifiers [84] due to their intrinsically much shorter pulse durations. The need for intricate and alignment-sensitive post-compression chains based on multipass (Herriott) cells [85] or nonlinear fibers [35] is eliminated, greatly simplifying the overall design. Moreover, the broader the generated output spectrum of the laser oscillator, the shorter the mid-IR wavelengths that can ultimately be reached ${ }^{4}$. This allows to address a larger set of molecules, with biomolecules like lipids and proteins facing strong absorption even below $4 \mu \mathrm{m}\left(>2500 \mathrm{~cm}^{-1}\right.$, see figure 1.1). Combining the few-cycle emission with high average powers and $\mathrm{MHz}$-repetition rates can thus eliminate the need for post-amplification, as will be demonstrated in chapter 5 .

\section{Direct diode-pumping}

Applications of coherent mid-infrared radiation such as FTIR-microscopy, FRS, or scattering-type scanning near-field optical microscopy (s-SNOM) [15] strongly benefit from high-efficiency and compact high-brightness light sources. In addition, a low-noise performance is desired since intensity fluctuations of the laser output will set an ultimate limit to the sensitivity of the measurements. Fortunately, all these characteristics are inherent to systems in which the gain medium can be pumped directly by semiconductor laser diodes. Even though there are several major challenges arising from the combination of direct diode-pumping and the technique of (soft-aperture) Kerr-lens mode-locking (KLM), a sophisticated pump setup and cavity design was realized and three laser systems could be successfully set up in the framework of this thesis.

\footnotetext{
4 The shortest wavelengths that can be generated via DFG are determined by the shortest $\left(\lambda_{1}\right)$ and longest $\left(\lambda_{2}\right)$ wavelength components of the incident laser pulse: $\lambda_{3}^{-1}=\lambda_{1}^{-1}-\lambda_{2}^{-1}$.
} 


\subsection{Thesis Outline}

This dissertation sheds new light on the question of how state-of-the-art solid-state laser systems can be improved to greatly advance downstream MIR applications. The focus lies on the first demonstration and further development of powerful diode-pumped femtosecond $\mathrm{Cr}^{2+}: \mathrm{ZnS} / \mathrm{ZnSe}$ laser oscillators based on KLM.

The thesis is organized as follows:

Chapter 2 lays the theoretical foundations of ultrashort pulse generation and introduces the basic concepts of soliton propagation and soliton mode-locking. First, the origin of laser pulse formation will be explained in section 2.1 together with the experimental technique of Kerr-lens mode-locking, which allows to generate few-cycle pulses directly from a laser cavity. In section 2.2 and 2.3, the principal factors influencing soliton dynamics are examined and summarized in the master equation of mode-locking. Finally, the effect of positive and negative net dispersion on the pulse formation process is addressed with respect to two specific mode-locking regimes.

Chapter 3 starts with introducing the advantages of $\mathrm{Cr}^{2+}$-doped II-VI gain media (section 3.1) and continues with examining the major challenges associated with direct diode-pumping (section 3.2). In section 3.3, the experimental results on the first directly diode-pumped $\mathrm{KLM} \mathrm{Cr}^{2+}$-doped II-VI oscillator are presented, highlighting the major breakthrough achieved upon soft-aperture KLM combined with a careful intra-cavity dispersion management. Moreover, measurements on the amplitude noise of the mode-locked output reveal a highly promising low-noise performance.

The subject of chapter 4 is to demonstrate the power-scalability of the results from chapter 3 , driven by the overall goal to reach and surpass the performance of state-of-the-art fiber-laser-pumped systems. In section 4.1, a focus is placed on the challenges when going towards higher intra-cavity peak powers with respect to mode-locking performance and pump setup design. The achieved output results will then be presented in section 4.2 , revealing the performance improvements over the first generation.

Chapter 5 focuses on pushing the ultimate limits of ultrafast diode-pumped $\mathrm{Cr}^{2+}$-doped II-VI lasers towards ever higher peak powers. The resulting modematching issues between pump and fundamental cavity beam are addressed in section 5.1, and section 5.2 reports a series of experimental findings on the cavity design and mode-locking performance as well as the achieved output results. The 
power of the third-generation system is finally illustrated through direct generation of mid-infrared radiation in a ZGP crystal (section 5.3), with a summary of the results achieved concluding this last experimental chapter (section 5.4).

In chapter 6, a summary is presented together with an outlook, highlighting and discussing future developments that are initiated by the achievements presented in the framework of this thesis. 


\section{FUNDAMENTALS OF ULTRAshort PUlse GENERATION}

The generation of few-cycle laser pulses - as emitted directly from a diode-pumped solid-state laser — is the result of a fascinating journey of mode-locked laser development, that has been started more than 50 years ago. In order to understand the series of linear and nonlinear mechanisms involved in the process, this chapter serves as a comprehensive introduction to the basic ideas behind ultrashort pulse generation (section 2.1), with a focus devoted to Kerr-lens mode-locking as well as solitary pulse propagation (section 2.2) and formation (section 2.3). The latter has become one of the most important pulse-shaping processes in nowaday's ultrafast laser systems, and is of great importance for the three laser systems presented in this thesis. In the following, the respective physical concepts will be explained and combined with mathematical derivations, based on the content of a series of excellent graduate-level textbooks [86-90].

\subsection{Formation of Laser Pulses}

Compared to other sources of radiation, lasers are devices that produce light of remarkable properties, ranging from high directionality and coherence to spectral purity and intensity. Broadly speaking, only three components are required to make a laser functional, namely (i) a gain medium for population inversion, (ii) a pump source to provide the energy needed for the inversion process, and (iii) a resonator structure that serves as an optical feedback mechanism for the amplification of light. More precisely, laser action is a result of stimulated emission in the gain medium, in which the power of incoming photons gets amplified during the pumping process. However, this requires more atoms of the medium to be excited into some higher quantum-mechanical energy level than are located in lower-lying states. As shown in figure 2.1, a 4-level system is characterized by radiative and fast nonradiative energy transitions, resulting in a sufficiently long lifetime of the upper laser level 2 and a short lifetime of the lower level 1. Hence, these kind of gain media are ideal to achieve a reasonably large population inversion and thus efficient laser action.

Typically, a classification is made based on the type of gain medium used and the temporal and spatial behaviour of the light emission. For the three lasers systems presented in this thesis, laser action is obtained by optically pumping a solid-state crystal with the emission of laser diodes. A part of the radiation of 
this solid-state laser is then coupled out through one of the end-mirrors (OC), eventually providing the coherent output beam for downstream applications. In addition, a laser is also characterized according to its longitudinal and transverse mode properties (see figure 2.1). As will be discussed in the next section, a multitude of longitudinal modes, which are electromagnetic plane waves that propagate in opposite directions and with different frequencies along the cavity axis, can oscillate within a laser resonator. Also, modes with different sizes and divergences can emerge from the cavity, being associated with the transverse electromagnetic mode $\left(\mathrm{TEM}_{m n}\right)$ intensity distributions [91]. However, for the single-transversal-mode lasers presented in this thesis, only the fundamental $\mathrm{TEM}_{00}$ mode is supported, and - as we shall see later - a good spatial overlap of the pump beam and the fundamental Gaussian mode inside the laser crystal is essential to maintain this mode of operation.

Up to now, only continuous-wave (CW) laser emission was considered, with the output power being nominally constant over time. In the next section, the concept of mode-locking together with its experimental techniques will be introduced, which is essential to realize a pulsed laser output.

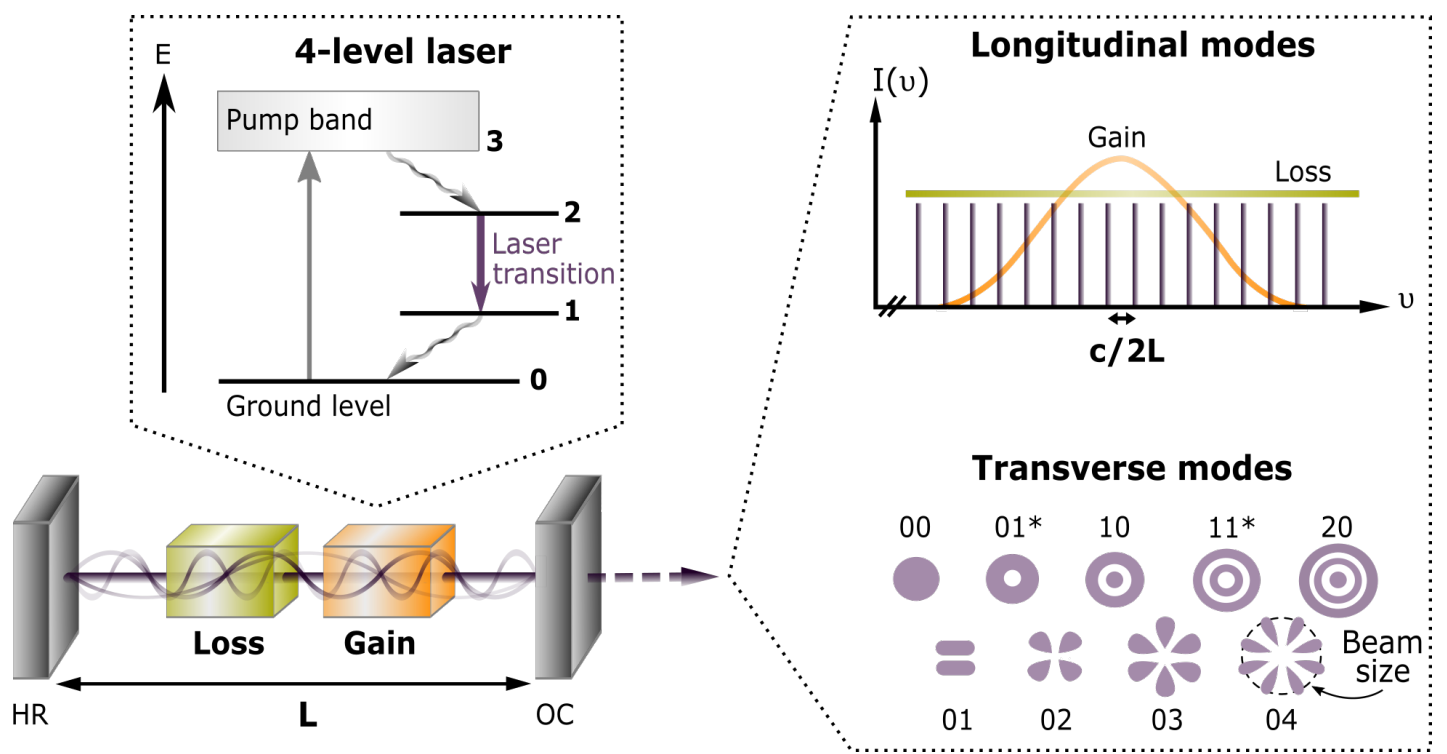

Fig. 2.1: Illustration of a two-mirror cavity of length L, including a highly-reflective (HR) mirror and a partially transmissive output coupler (OC). The energy diagram of the gain medium is represented by a simplified 4-level structure, which involves radiative transitions and non-radiative decays (denoted as wavy arrows). Also depicted are cylindrical transverse and longitudinal mode distributions supported by the resonator. In the frequency domain, the standing waves of the cavity are separated by $\Delta \nu=c / 2 L$, and only a certain number of modes gets amplified when the gain of the lasing medium exceeds the resonator losses. By contrast, the transverse modes refer to the electromagnetic field patterns in the spatial domain. 


\subsubsection{Locking the Longitudinal Modes of a Laser Cavity}

In the very general case of a standing-wave cavity made from two parallel reflecting surfaces, only a specific set of resonant frequencies $\nu_{n}$ is supported, since the standing waves are forced to form nodes at the end-mirrors:

$$
\nu_{n}=\frac{c}{\lambda}=\frac{c}{2 L} n \quad(n \in \mathbb{N}) .
$$

Here, $c$ is the speed of light, $L$ indicates the length of the cavity, and $n$ is the mode number. As depicted in figure 2.1, the frequency difference between two consecutive modes is $\Delta \nu=c / 2 L=1 / t_{r e p}$ and is given by the inverse of the cavity round-trip time $1 / t_{\text {rep }}$. In principle, an infinite number of standing waves could oscillate within the resonator, but, depending on the gain bandwidth of the laser medium, only a certain fraction experiences sufficient gain to account for losses. For a free-running laser, there is no fixed mode-to-mode phase and amplitude relationship and the total electric field $E(t)$ can therefore be described as [87]:

$$
E(t)=\sum_{n=-m}^{n=m} E_{n} e^{i\left(w_{0}+n 2 \pi / t_{r e p}\right) t+i \varphi_{n}},
$$

where $\varphi_{n}$ and $E_{n}$ correspond to the electric field and phase of the $n$th mode, with $w_{0}$ being the mode frequency at the center of the gain bandwidth. Consequently, the output laser intensity fluctuates in time and, at first sight, it looks like a completely random spiking behaviour with the mode phases varying between $-\pi$ and $+\pi$. However, since the spikes originate from the sum of $N=2 m+1$ evenly spaced frequency components, the resulting waveform reveals some characteristics of a Fourier series. As illustrated in figure 2.2 (a), it has some kind of periodicity with a period of $1 / \Delta \nu$, with each spike having a duration of roughly $1 / N \Delta \nu[86]$.

However, in order to generate temporally well-behaved output pulses, the modes have to be "coupled" together. In a process referred to as mode-locking, the spectral ampltiudes and phases get correlated with each other, and equation 2.2 evolves into [87]:

$$
E(t)=E_{0} \sum_{n=-m}^{n=m} e^{i\left(w_{0}+n 2 \pi / t_{r e p}\right) t}=E_{0}\left[\frac{1-e^{i N\left(2 \pi / t_{r e p}\right) t}}{1-e^{i\left(2 \pi / t_{r e p}\right) t}}\right] e^{i w_{0} t} .
$$

For this, all modes were assumed to have equal field strengths $E_{n}=E_{0}$ and, by a shift of our choice of time, we have also set $\varphi_{n}=\varphi_{0}=0$. The corresponding output intensity is then simply the product of the electric field $E(t)$ with its complex conjugate $E^{*}(t)$ :

$$
I(t)=|E(t)|^{2}=E(t) E^{*}(t)=E_{0}^{2} \frac{\sin ^{2}\left(N \pi t / t_{r e p}\right)}{\sin ^{2}\left(\pi t / t_{r e p}\right)} .
$$


(a) random phases
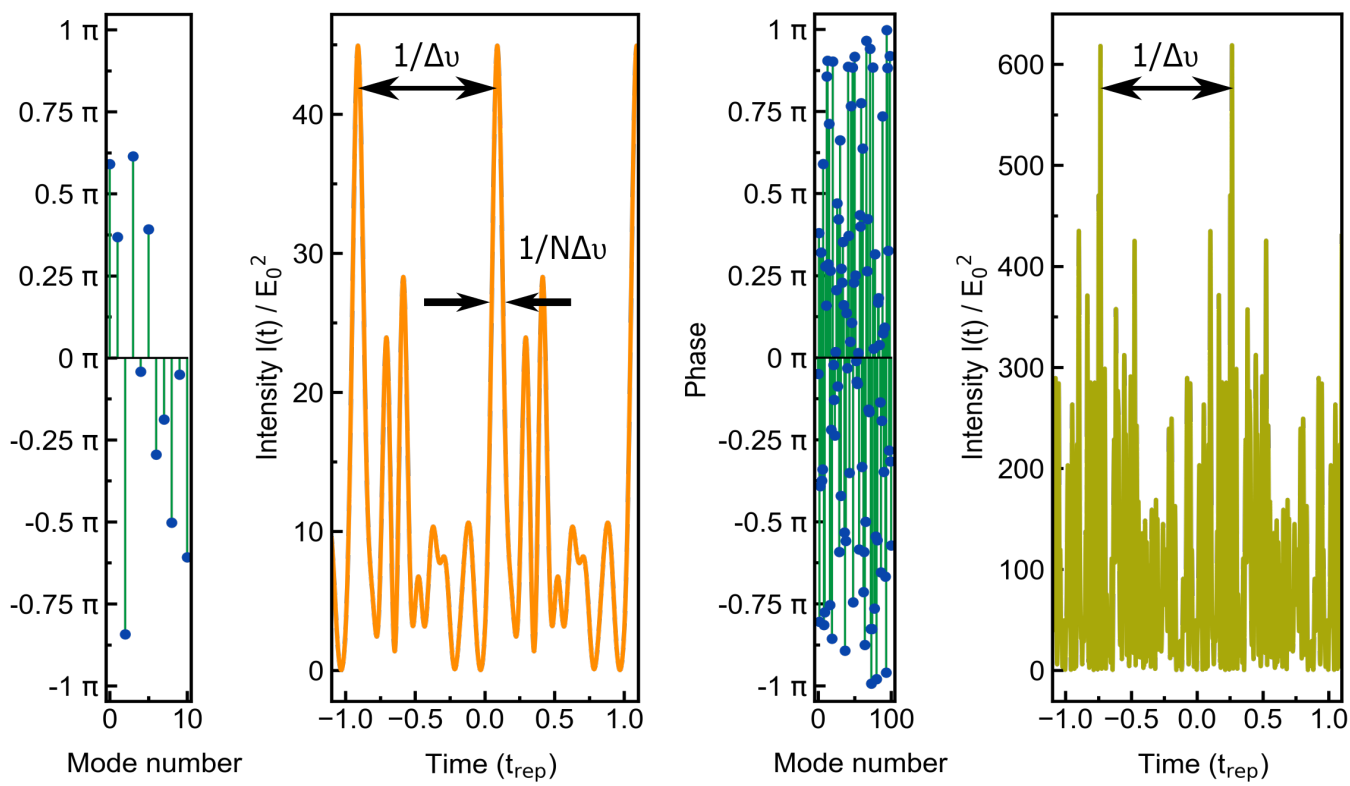

\section{(b) locked phases}
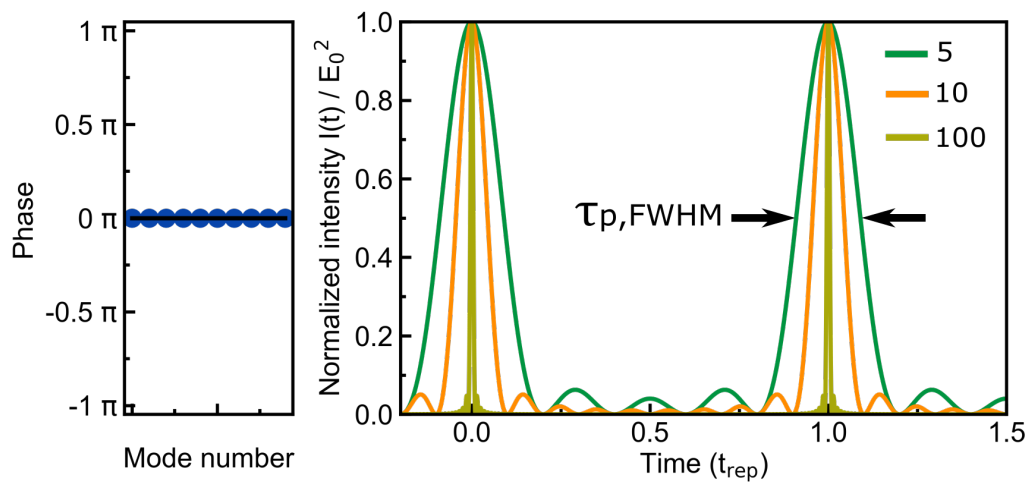

Fig. 2.2: Simulated temporal behaviour of the squared amplitude of the total electric field for the case of 10 and 100 oscillating modes (a), all having the same amplitude $\mathrm{E}_{0}=1$ but random phases. The spike with the highest intensity will experience the highest gain during a cavity roundtrip and will eventually lead to the initiation of the mode-locking process. For simplicity, the amplitudes were assumed to be constant (i.e. flat gain), which is not the case in reality. Note that the response time of conventional photodetectors is typically too slow to resolve this complex behaviour in the temporal domain. Instead, its average value is measured. (b) Same parameters as before, but now for the case of 5, 10, and 100 modes having constant phases. The more modes are oscillating, the narrower the FWHM, the higher the amplitude, and the longer the dead time in between. Note that the intensities were normalized to an interval of $[0,1]$.

The result is a train of short but intense pulses separated by the round-trip time $t=t_{r e p}$, and with a FWHM pulse duration given approximately by $t_{p} \approx$ $t_{r e p} / N \approx 1 / \Delta \nu_{g}$, where $\Delta \nu_{g}$ is the gain bandwidth of the laser [87]. As depicted in 
figure 2.2 (b), the main peaks are accompanied by $N-2$ much weaker subsidiary peaks, and the larger the number of oscillating modes, the shorter will be the generated pulse duration. In chapter 3, we will see that the first diode-pumped laser system presented in this thesis, MIRanda1, is able to emit pulses as short as $45 \mathrm{fs}$ at a repetition rate of $65 \mathrm{MHz}$. With an output spectral bandwidth $(3 \mathrm{~dB})$ of about $185 \mathrm{~nm}(10.04 \mathrm{THz})$ at a central wavelength of $2.35 \mu \mathrm{m}$, an impressively large number of $N=10.04 \mathrm{THz} / 65 \mathrm{MHz} \approx 154461$ modes are thus locked in phase.

\subsubsection{Experimental Realization Methods}

In general, different techniques exist to force the laser into pulsed operation. They have in common that a fixed-phase relationship among the longitudinal modes is established by introducing some kind of mode modulation, with the modulation frequency being linked to the repetition rate of the laser cavity. Here, locked modes in the form of a more intense radiation peak suffer lower propagation losses inside the resonator than any other combination of (unlocked) modes, and are therefore favoured.

Typically, a distinction is made between active and passive methods. Active mode-locking was first theoretically described by Kuizenga and Siegman [92] and requires the resonator losses to be modulated by an externally driven amplitude (AM) or phase (FM) modulator. However, the commonly used electro-optic or acousto-optic component adds to the cost and complexity of the setup since the modulation frequency must be matched precisely to the spacing of the cavity modes Also, the external modulation is limited to electronic speed, therefore constraining the minimum attainable pulse duration ( ps-range).

These drawbacks can be overcome by passive mode-locking techniques, which do not require an external source but only rely on the nonlinear loss modulation triggered by the laser pulse itself. This modulation is automatically synchronized with the travelling pulses, and is accomplished by incorporating a saturable absorber into the laser cavity, whose absorption decreases with increasing intensity. A very prominent example are the semiconductor saturable absorber mirrors (SESAMs) [93] that have found widespread use in research and industry. They can be properly taylored for optimal performance with respect to the magnitude of modulation, the time needed for the system to recover, and the radiation fluence required to bring about the absorption saturation effect.

Rather than exploiting real saturable absorption, the few-cycle laser pulses genereated in the framework of this thesis exploit so-called artificial saturable absorber action based on the electronic Kerr-effect. First demonstrated and explained in 1991 [58, 94], the Kerr-lens mode-locking (KLM) technique has enabled 
the generation of one of the shortest pulses directly from a laser cavity, with pulse durations of less than two optical cycles [95]. Similarly short pulse durations could also be obtained upon SESAM-assisted Kerr-lens mode-locking [96], however, pure KLM operation allows for a fast saturable-absorber-like action with almost instantaneous relaxation, response and no real absorption.

\section{Kerr-lens mode-locking}

The general principle behind KLM relies on a beam self-focusing effect arising from a third-order $\chi^{(3)}$-nonlinearity inside a nonlinear medium. When the electric field of a laser $E(t)$ is applied to some medium, the existing dipoles are aligned along the direction of the incoming field or new electric dipoles are induced. The resulting effect is termed polarization $P(t)[81,97]$ and can be expressed by a linear $P^{(1)}(t)$ and nonlinear term $P^{N L}(t)$ in a Taylor-series expansion:

$$
\begin{aligned}
P(t) & =\epsilon_{0}\left(\chi^{(1)} E(t)+\chi^{(2)} E(t) E(t)+\chi^{(3)} E(t) E(t) E(t)+[\ldots]\right) \\
& =\epsilon_{0} \chi^{(1)} E(t)+\epsilon_{0} \sum_{n=2}^{\infty} \chi^{n} E(t)^{n} \\
& \equiv P^{(1)}(t)+P^{N L}(t),
\end{aligned}
$$

where $\chi^{n}$ is referred to as the nonlinear optical susceptibility ${ }^{1}$ of order $n$ and $\epsilon_{0}$ is the vacuum permittivity. For the power levels considered in this thesis, we only consider $\chi^{(2)}$ - and $\chi^{(3)}$-related processes, and an overview of some related optical phenomena is given in table 2.1 [81, 97-99].

The Kerr effect is a third-order $\left(\chi^{(3)}\right)$ effect and originates from the intensity dependence of a material's refractive index. It can be written as [81]:

$$
n(I)=\sqrt{1+\chi^{(1)}}+\frac{3}{4} \frac{\chi^{(3)}}{\epsilon_{0} c n_{0}^{2}} \cdot I \equiv n_{0}+n_{2} \cdot I,
$$

where $n_{0}$ and $n_{2}$ are the linear and nonlinear refractive indices, respectively. Since the intensity is highest in the center of a laser pulse, this part will experience a higher refractive index than the wings in transverse direction, leading to a focusing effect in media with $n_{2}>0$. For pulses with peak power $P_{0}$ and $\left(1 / e^{2}\right)$-beam radius $w$, this Kerr-lens has an approximate focal length of [100]: ${ }^{2}$

$$
f_{K e r r}=\frac{\pi w^{4}}{4 n_{2} P_{0} L},
$$

1 Note that the susceptibility has the form of a tensor, and $\chi^{(2)}$ and $\chi^{(3)}$ are tensors of the third and fourth rank, respectively.

2 Equation 2.7 is only valid for a circular beam with $w_{x}=w_{y}=w$. If $w_{x} \neq w_{y}$, both directions will experience a different strength of focusing. 
with $L$ denoting the length of the medium. When adding a Kerr lens into a laser cavity, the laser mode diameter for pulsed operation changes but does not automatically impose a preference for mode-locked operation. Only the combination of self-focusing together with an intra-cavity aperture provides a fast saturable absorber-like action resulting in a so-called self-amplitude modulation (SAM, see section 2.3.2) [101].

\begin{tabular}{lcc} 
Nonlinear process & & Susceptibility \\
\hline Second-harmonic generation & SHG & $\chi^{(2)}(2 \omega ; \omega, \omega)$ \\
Sum frequency generation & SFG & $\chi^{(2)}\left(\omega_{3} ; \omega_{1}, \omega_{2}\right)$ \\
Difference frequency generation & DFG & $\chi^{(2)}\left(\omega_{2} ; \omega_{3},-\omega_{1}\right)$ \\
Optical rectification & OR & $\chi^{(2)}(0 ; \omega,-\omega)$ \\
\hline Kerr effect (nonlinear refr. index) & & $\chi^{(3)}(\omega ; \omega,-\omega, \omega)$ \\
Four-Wave Mixing & FWM & $\chi^{(3)}\left(\omega_{4} ; \omega, \omega, \omega, \omega_{3}\right)$ \\
Third-Harmonic Generation & THG & $\chi^{(3)}(3 \omega ; \omega, \omega, \omega)$ \\
Two-Photon Absorption & TPA & $\operatorname{Im}\left[\chi^{(3)}(\omega ; \omega, \omega,-\omega)\right]$
\end{tabular}

Table 2.1: Important second- and third-order nonlinear optical susceptibilities and corresponding nonlinear processes. The first argument in the susceptibility represents the frequency of the generated wave, while the terms after the semicolon are related to the frequencies of the incoming waves, with $\omega_{3}=\omega_{1}+\omega_{2}$ and $\omega_{4}=\omega_{1}+\omega_{2}+\omega_{3}$.
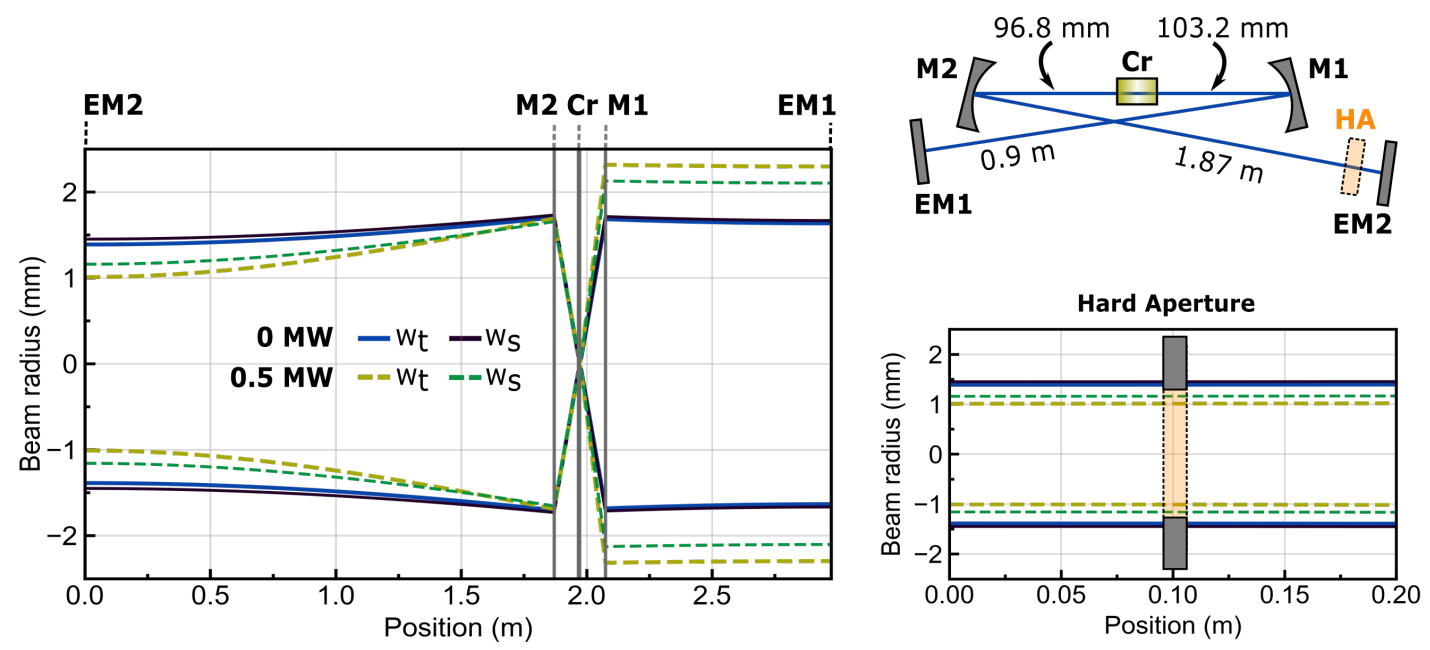

Fig. 2.3: Using the nonlinear ABCD-model described in section 4.1.2, the intra-cavity beam radius of an asymmetrical x-folded cavity, similar to MIRanda2, was calculated for two different peak powers. The self-focusing Kerr effect takes place inside the $5 \mathrm{~mm}$ long Cr:ZnS crystal $(\mathrm{Cr})$, which is surrounded by the spherical mirrors M1 and M2. The angle of incidence on the latter was assumed to be $2^{\circ}$ and the arm lengths correspond to a repetition rate of $50 \mathrm{MHz}$. To initiate hard-aperture KLM, a suitable position was identified close to the end-mirror EM2 in the long arm, where the diameter of the high-power beam is decreased. 
As shown in figure 2.3, one could place a physical hard aperture into the beam path to induce higher losses for continuous-wave operation. On the other hand, the Kerr effect inside the gain crystal results in an intensity-dependent gain distribution and can lead to a better overlap between the pump and fundamental laser mode [102] (figure 2.4). As will be demonstrated in the experimental chapters, this soft-aperture KLM technique is a very elegant way to start and stabilize the mode-locking mechanism, in which the crystal plays both the role of a gain and Kerr medium.

However, this additional nonlinear lensing is only drastically enhanced when the cavity is operated close to one of the stability zone boundaries. In this respect, a useful parameter is the so-called Kerr strength, which is a measure of the effect of the Kerr lens on the laser cavity. It can be expressed as the change in cavity mode size due to a small increase in the intra-cavity peak power $P_{0}[103,104]$ :

$$
\gamma_{K e r r}=\left(\frac{1}{\omega_{c w}} \frac{d \omega}{d p}\right)_{p=0},
$$

where $\omega_{c w}$ refers to the continuous-wave mode size $\left(P_{0}=0\right)$ at a given plane inside the oscillator and $p=P_{0} / P_{c r}$ is the normalized power with $P_{c r}$ being the critical power for self-focusing. The analytic form of the latter has been shown to be of the form $P_{c r}=\alpha\left(\lambda^{2} / 4 \pi n_{0} n_{2}\right)$, where $\alpha$ is a material-independent constant ${ }^{3}$ [105].
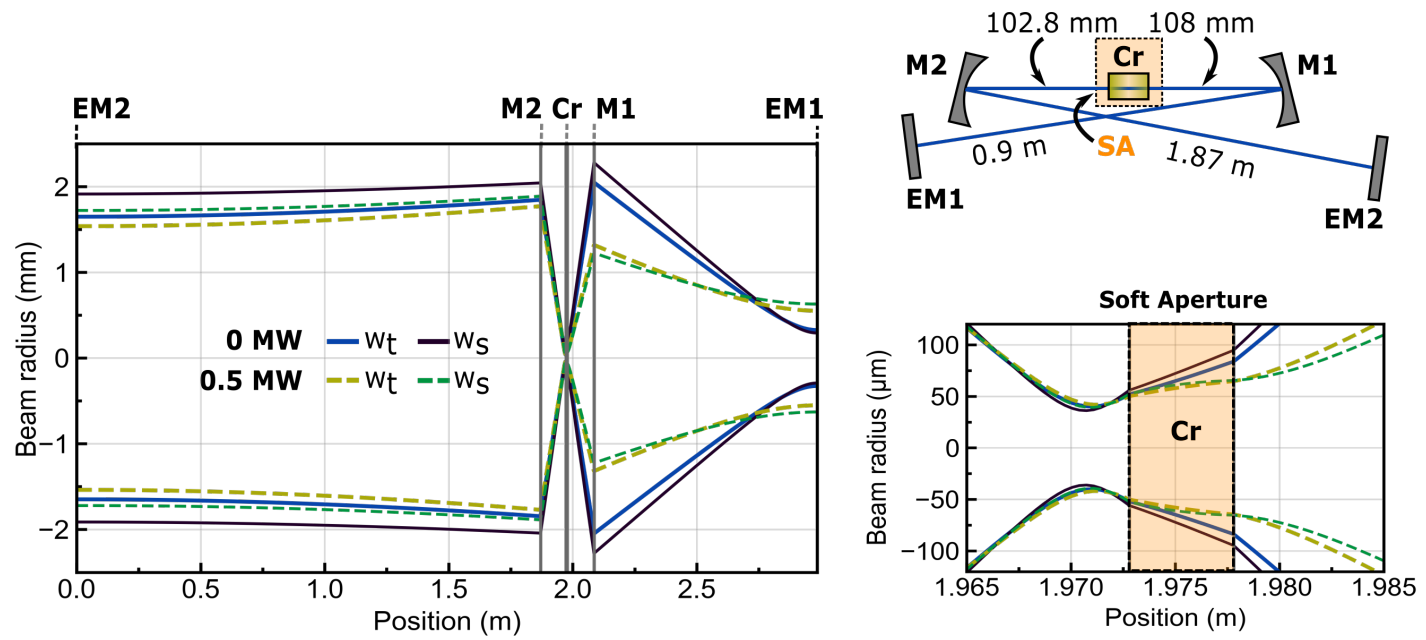

Fig. 2.4: Same parameters as in figure 2.3 but with different distances between the curved mirrors to illustrate soft-aperture KLM. In this case, the beam diameter of the high-power beam is slightly decreased inside the gain medium. Depending on the pump beam geometry, this can lead to a better overlap between the pump and fundamental cavity beam, therefore favoring pulsed operation.

${ }^{3}$ In [105], a theoretical lower bound for self-focusing in bulk media was found to be $\alpha \approx 1.86225$. 

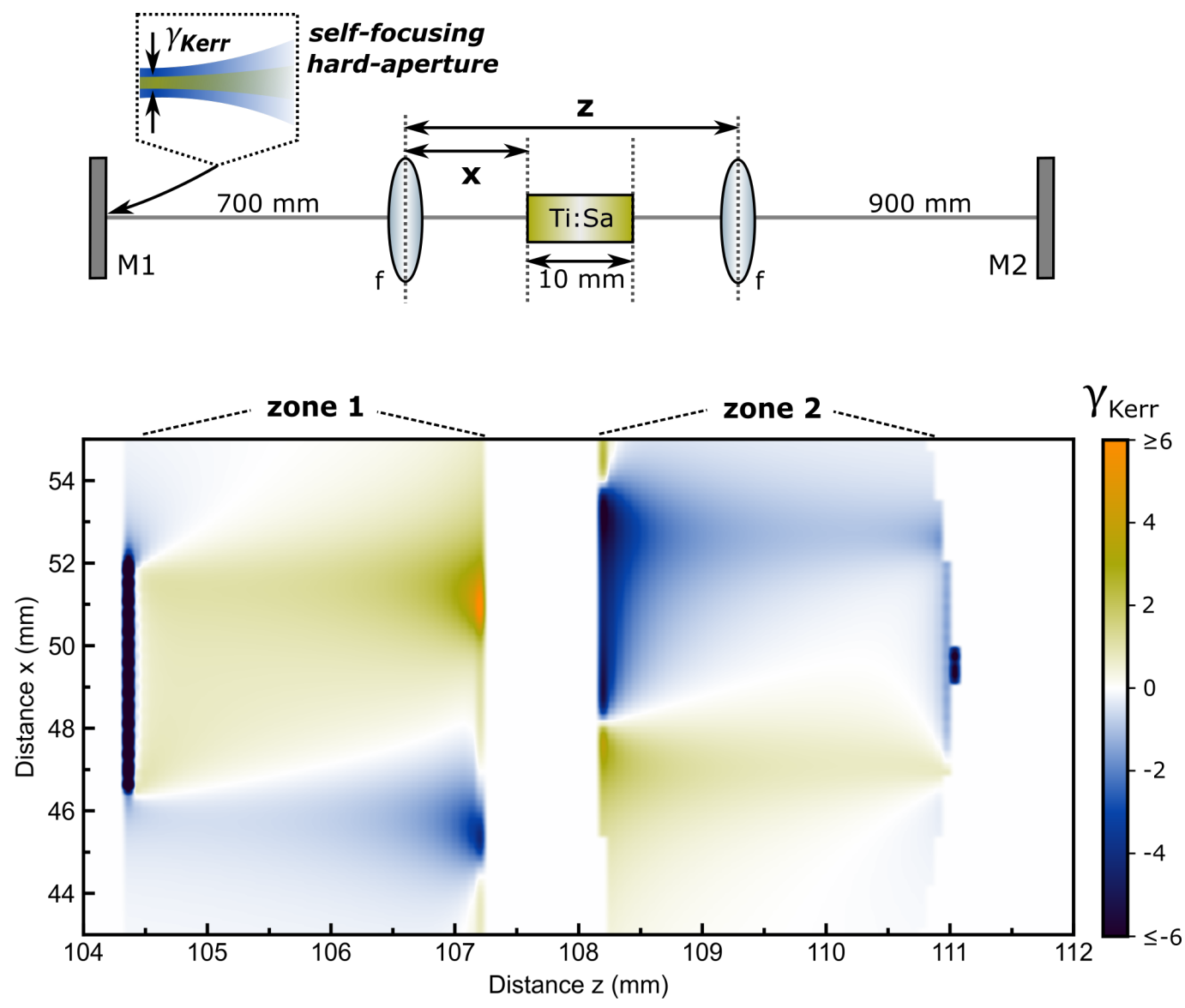

Fig. 2.5: Two-dimensional simulation of the Kerr strength parameter for varying crystal positions $x$ and distances $z$ between the focusing lenses $(\mathrm{f}=50 \mathrm{~mm})$. The $\gamma_{K e r r^{-v a l u e s}}$ are calculated for spot sizes on mirror M1, revealing the two stability zones of a typical laser resonator.

When going to higher and higher intra-cavity peak powers, suitable locations for a soft (or hard) aperture need to be identified to still ensure sufficient SAMaction. Therefore, the variation of $\gamma_{K e r r}$ will be investigated in more detail for the second-generation laser system, MIRanda2, which was meant to be optimized for higher power levels. More information on the performed nonlinear ABCDmatrix simulations that were also used to plot figures 2.3 and 2.4 can be found in chapter 4.1.2. To provide a first impression of the Kerr strength variations for different gain medium positions and curved mirror separations, a typical Ti:sapphire laser resonator was simulated based on the parameters presented in reference [103]. The result is plotted in figure 2.5 and is in close agreement with the simulation presented by Magni et al.. Small deviations are most likely due to uncertainties in the simulation parameters as well as the slightly different approach used for modeling the Kerr-lensing effect in the gain medium (see section 4.1.2). Nonetheless, this example serves to illustrate the typical operation characteristics of a Kerr-lens 
mode-locked laser: (i) To obtain a significant nonlinear loss modulation for KLM, the laser cavity needs to be operated close to the stability zone boundaries, at which the Kerr strength and thus the nonlinear effects are enhanced. (ii) Moreover, zero values not only indicate positions of constant beam sizes but also denote unstable laser configurations, revealing the two characteristic zones of stability. (iii) Also, the crystal position needs to be adjusted, which can result in either decreasing $\left(\gamma_{\text {Kerr }}<0\right)$ or increasing $\left(\gamma_{\text {Kerr }}>0\right)$ beam diameters upon pulsed operation. Hence, these two-dimensional simulations provide a useful guideline for oscillator design and optimization.

Before we conclude this part, it should be noted that the intensity of a laser pulse is not only a function of space but also time. As will be seen in the following section 2.2.2, another important effect termed self-phase modulation (SPM) plays a crucial role.

\subsection{Soliton Pulse Propagation}

The concept of solitons already came up more than a century ago [106], and it would be difficult to find a field of theoretical physics where it is not used for explaining important phenomena, including the fields of hydrodynamics, particle physics and astronomy. In laser physics, the soliton regime allows to generate und sustain the shortest achievable pulses, which are referred to as temporal optical solitons or fundamental solitons. Upon propagation along nonlinear dispersive media, their temporal and spectral shape remains unchanged through a proper balance of group velocity dispersion (GVD) and self-phase modulation (SPM) and negligible losses.

\subsubsection{Dispersion}

In mode-locked lasers, an abundance of frequency components is travelling around, each of them experiencing a different change in absolute phase $\phi(w)$ when passing through material or being reflected by specific mirrors (see section 3.3.2). The phaseterm $\phi(w)$ is typically expanded in a Taylor series around the central frequency $w_{0}$ of the pulse [107, 108]:

$$
\phi(w)=\sum_{m=0}^{\infty} \frac{\left(w-w_{0}\right)^{m}}{m !} \underbrace{\left(\frac{\partial^{m}}{\partial w^{m}} \phi(w)\right)}_{D_{m}}(m \in \mathbb{N}) .
$$

Together with the relationship between the phase and the wave vector $k(w), \phi(w)=$ $k(w) z=w n(w) z / c$, the coefficients $D_{m}$ provide a means to describe the effect of dispersion on a pulse: 
- $\mathbf{D}_{0}$ : an overall phase accumulation.

- $\mathbf{D}_{\mathbf{1}}(\mathbf{G D})$ : the group delay (GD) denotes a temporal shift of the pulse with respect to a pulse propagating in free space, and contains the inverse of the group velocity $1 / v_{g}=\partial k / \partial w$.

- $\mathbf{D}_{\mathbf{2}}(\mathbf{G D D})$ : the group delay dispersion (GDD) term is the most famous term. It refers to the variation of the GD with respect to $w$ and is usually specified in $\mathrm{fs}^{2}$ for a given length of a medium. The so-called group velocity dispersion $\left(\mathrm{GVD}, \partial^{2} k / \partial w^{2}\right)$ is the GDD per unit length $\left(\mathrm{fs}^{2} / \mathrm{mm}\right)$. In table 2.2 , the GVDvalues of selected materials relevant for this thesis are listed, with GVD $>0$ indicating positive (normal) dispersion, while substrates with GVD $<0$ are said to exhibit negative (anomalous) dispersion.

- $\mathbf{D}_{\mathbf{3}}(\mathbf{T O D}), \mathbf{D}_{4}, \mathbf{D}_{\mathbf{5}}, \ldots$ : third- and other higher-order dispersion terms. For narrowband laser pulses, they can often be neglected, however, third-order dispersion (TOD) becomes significant when going to even shorter pulse durations. Therefore, specifically taylored dispersive mirrors are needed to account for TOD in few-cycle laser systems (see section 3.3.2).

\begin{tabular}{lccrr}
\hline material & $\mathbf{n}_{0}$ & $\mathbf{n}_{2}$ & GVD & Ref. \\
\hline $\mathbf{Z n S}$ & 2.26 & $<100^{*}(@ 1.95 \mu \mathrm{m})$ & +128 & {$[109]$} \\
$\mathbf{Z n S e}$ & 2.44 & $\sim 100(@ 1.95 \mu \mathrm{m})$ & +218 & {$[110,111]$} \\
YAG $\left(\mathrm{Y}_{3} \mathrm{Al}_{5} \mathrm{O}_{12}\right)$ & 1.80 & $8 \pm 2(@ 1.3 \mu \mathrm{m})$ & -124 & {$[112,113]$} \\
Sapphire $\left(\mathrm{Al}_{2} \mathrm{O}_{3}\right)$ & 1.73 & $3.3(@ 1.55 \mu \mathrm{m})$ & -210 & {$[114,115]$} \\
Fused silica $\left(\mathrm{SiO}_{2}\right)$ & 1.43 & $2.8 \pm 0.1(@ 1.55 \mu \mathrm{m})$ & -180 & {$[116,117]$} \\
$\mathbf{M g F}_{2}$ & 1.37 & $1.1 \pm 0.2(@ 1.06 \mu \mathrm{m})$ & -61 & {$[118,119]$} \\
$\mathbf{B a F}_{2}$ & 1.46 & $2.08-3.22(@ 0.532 \mu \mathrm{m})$ & -14 & {$[120,121]$} \\
$\mathbf{Z G P}\left(\mathrm{ZnGeP}_{2}\right)$ & 3.14 & $\sim 550(@ 2.2 \mu \mathrm{m})$ & +511 & {$[122,123]$} \\
\hline
\end{tabular}

Table 2.2: Linear and nonlinear $\left(\left(\mathrm{cm}^{2} / \mathrm{W}\right) \cdot 10^{-16}\right)$ refractive index of selected materials and their group velocity dispersion $\left(\mathrm{fs}^{2} / \mathrm{mm}\right)$. Unless otherwise stated, the values correspond to a wavelength of $2.3 \mu \mathrm{m}$. * Estimated value based on $\mathrm{ZnSe}$.

Controlling chromatic dispersion in a laser cavity is a major challenge in ultrashort pulse generation since every optical element - and even air - is dispersive. To include the effect of dispersion on a laser pulse, its $(1+1)$-dimensional field envelope $A(z, \tau)$ can be described in the local pulse-frame $\tau=t-z / v_{g, w_{0}}$, traveling with the central group velocity $v_{g, w_{0}}$ and along the propagation distance $z \in\left[0, N \cdot L_{\text {cav }}\right]$ [124]. Here, $L_{\text {cav }}$ is the length corresponding to one complete round-trip through the cavity, and $N$ is the number of round-trips. To model the propagation and formation of pulses, a slowly-varying envelope approximation is typically employed [125]. It implies that the change of the pulse amplitude during an optical cycle is negligible when compared to the magnitude of the amplitude itself. Moreover, it can 
be applied down to the single-cycle regime, when some additional corrections are included [126]. The effect of dispersion on the laser field envelope is then described as [124]:

$$
\frac{\partial A(z, \tau)}{\partial z}=i \frac{D_{2}}{2} \frac{\partial^{2} A(z, \tau)}{\partial \tau^{2}}+[\ldots]
$$

where the square brackets refer to the higher-order dispersion terms.

\subsubsection{Self-Phase Modulation}

As we have seen in section 2.1.2, the material's refractive index does not only depend on frequency but also intensity. Besides the explained effect of spatial beam self-focusing, the time-dependent intensity $I(t)$ of a laser pulse can thus result in modulations of the laser pulses' temporal phase $\Phi(t)[81,90]$ :

$$
\Phi(t)=w_{0} t-\frac{2 \pi}{\lambda_{0}} n(I) L .
$$

Consequently, the instantaneous frequency $w(t)$ of a laser pulse changes as:

$$
w(t)=\frac{d \Phi}{d t}=w_{0}-\frac{2 \pi}{\lambda_{0}} \frac{d n(I)}{d t} L=w_{0}\left(1-\frac{n_{2}}{c} \frac{d I(t)}{d t} L\right) .
$$

To visualize the effect on the temporal and spectral intensity distribution of a laser pulse, spectrograms were calculated for different pulse peak intensities (see figure 2.6). A spectrogram is the time convolution of the pulse electric field $E(t)$ with a variable-delay gate function $g(t-\tau): S(w, \tau)=\left|\int_{-\infty}^{+\infty} E(t) g(t-\tau) \exp (-i w t) d t\right|^{2}$ $[127,128]$, and resembles the outcome of a cross-correlation frequency-resolved optical gating (XFROG) setup [129]. Here, two pulses - a Fourier-transform limited gate pulse and a probe pulse - perform sum-frequency generation (SFG) inside a nonlinear medium, with one pulse being variably delayed with respect to the other. In the performed simulations, both pulses had a central wavelength of $2300 \mathrm{~nm}(130 \mathrm{THz})$, corresponding to a sum-frequency of $260 \mathrm{THz}$. The gate pulse duration was set to be Fourier-transform limited (40 fs), however, the phase of the second (probe) pulse was changed to investigate the effects of either pure SPM (figure 2.6 (a) and (b)) or negative GDD (figure 2.6 (c) on the shape of the spectrogram. For the sake of illustration, the additional chirp acquired by material dispersion was neglegted in (a) and (b). In addition, the marginals are plotted on the sides, and are obtained through integration over the delay and frequency axis, respectively. When comparing the two spectrograms related to SPM with the one of a negatively chirped pulse, one can see that the effect of negative GDD can account for the linear (positive) chirp in the center of a SPM-broadened pulse. 


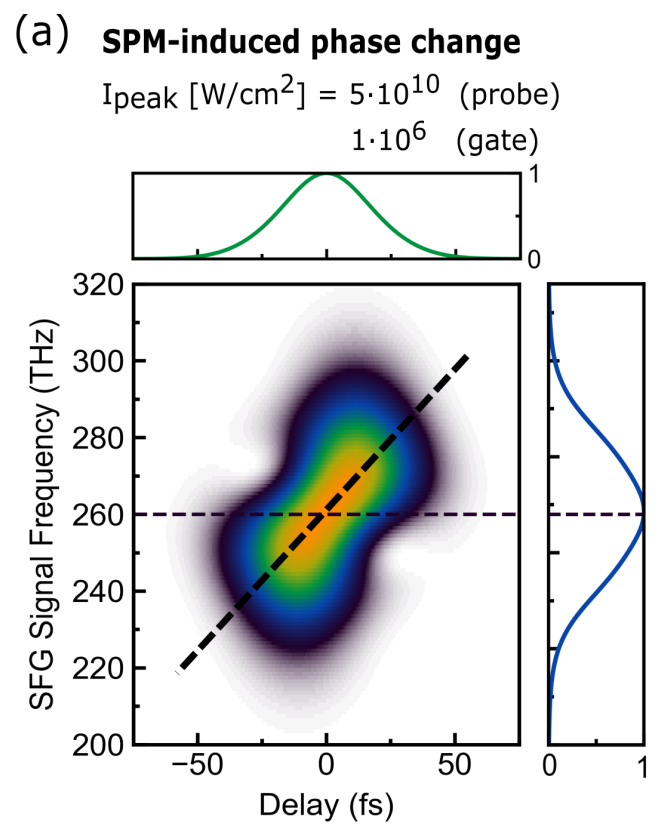

\section{(b) SPM-induced phase change}
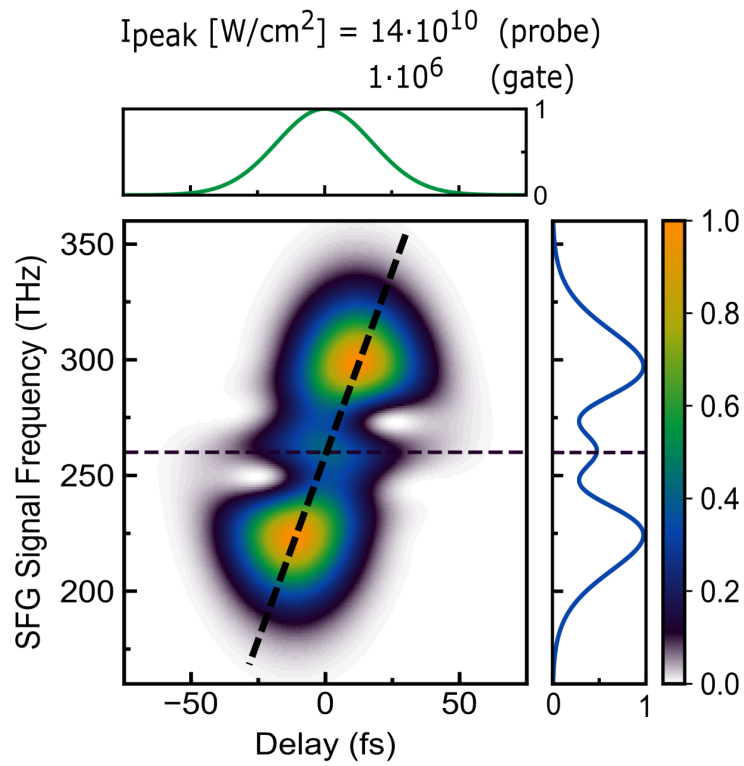

(c) GDD-induced phase change $\mathrm{GDD}\left[\mathrm{fs}^{2}\right]=-1000$

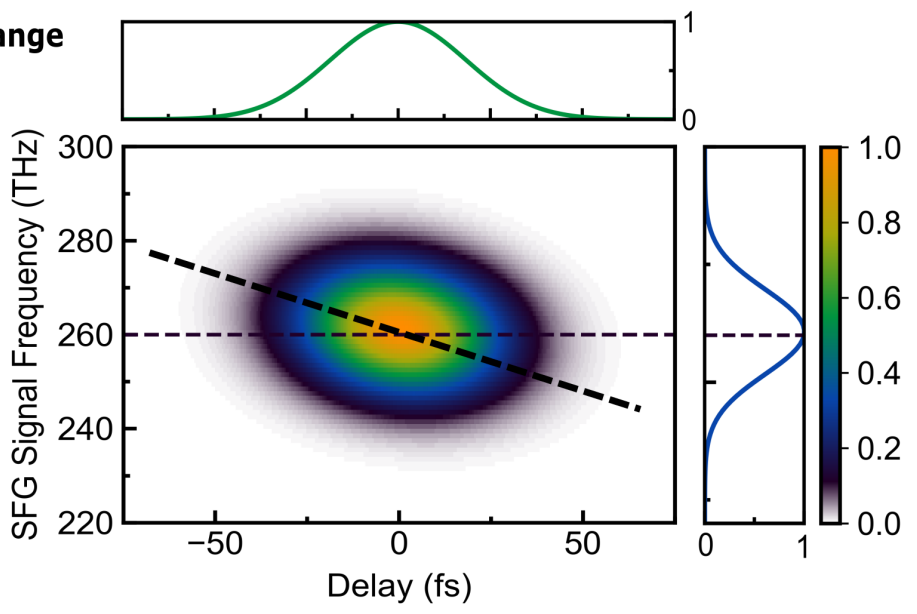

Fig. 2.6: Simulated spectrograms to illustrate the effect of SPM and negative GDD on the temporal and spectral pulse shape. (a,b) SPM-induced nonlinear phase change arising from different peak intensities of the probe and gate pulse when passing through a $2 \mathrm{~mm}$ thick ZnSplate. The higher the peak intensity, the stronger the frequency modulation in the spectrogram. The temporal pulse envelope is, however, not directly affected by SPM. Also, the tilted black dashed lines indicate that the chirp in the center of the SPM-broadened pulse is (mostly) linear. (c) To only model the effect of second-order dispersion, the temporal phase of the probe pulse was changed according to [130], and results in a linear chirp of opposite sign. Therefore, negative GDD can account for the linear (positive) chirp in the center of a SPM-broadened pulse.

Interestingly, strong self-phase modulation can lead to pronounced oscillations in the optical spectrum, as shown in the frequency marginal of (b). According to equa- 
tion 2.12, SPM changes the instantaneous frequency of the pulse as $\Delta w \propto d I(t) / d t$. Therefore, there are two distinct points in time that take the same frequency value. Depending on their relative phase difference, constructive or destructive interference takes place and results in the observed multipeak structure [107].

Finally, to also include the effect of self-phase modulation on the laser field envelope $A(z, \tau)$, the following expression is used [124]:

$$
\frac{\partial A(z, \tau)}{\partial z} \approx-i \gamma|A(z, \tau)|^{2} A(z, \tau)
$$

Here, $\gamma$ is the so-called SPM coefficient and can be estimated as $\gamma=8 \pi^{2} n_{0} n_{2} / \lambda^{2}$ for the case of strong beam focusing inside a nonlinear medium.

\subsubsection{The Fundamental Soliton}

When considering only the effects of GDD and SPM, the propagation of laser pulses can be described by an equation that is known as the nonlinear Schrödinger equation $(\mathrm{NLSE})^{4}[131-133]$ :

$$
\frac{\partial A(z, \tau)}{\partial z}=i \frac{D_{2}}{2} \frac{\partial^{2} A(z, \tau)}{\partial \tau^{2}}-i \gamma|A(z, \tau)|^{2} A(z, \tau) .
$$

For the case of negative dispersion with $D_{2}<0$, the NLSE supports a stable solution - the unchirped fundamental soliton [65, 107, 131]:

$$
A(z, \tau)=\sqrt{P_{0}} \cdot \operatorname{sech}\left(\frac{\tau}{\tau_{0}}\right) e^{i \gamma P_{0} z / 2} .
$$

Its amplitude exhibits a characteristic hyperbolic secant profile with peak power $P_{0}=$ $\left|A_{0}(z, \tau)\right|^{2}$, while the soliton's temporal (and spectral) shape remains unchanged upon propagation. The full width at half maximum (FWHM) of the soliton's pulse width can then be calculated as $\tau_{F W H M} \approx 1.76 \cdot \tau_{0}$ [107]. In order to obtain a stable solution, the soliton peak power and pulse duration are related to the dispersion and nonlinearity via the area theorem ${ }^{5}[107,131]$ :

$$
\tau_{0}=\sqrt{\frac{\left|D_{2}\right|}{\gamma P_{0}}} .
$$

\footnotetext{
4 It is very similar to the Schrödinger equation in quantum mechanics that describes how a wave function spreads out in space as time goes by.

5 For a particular pulse solution to exist, certain conditions must be satisfied that are expressed in the form of so-called area theorems [134].
} 
Hence, when the effects of both SPM and negative dispersion are properly balanced, the optical bandwidth of an initially unchirped pulse is left unaffected and a stable soliton pulse is formed as shown in figure 2.7.

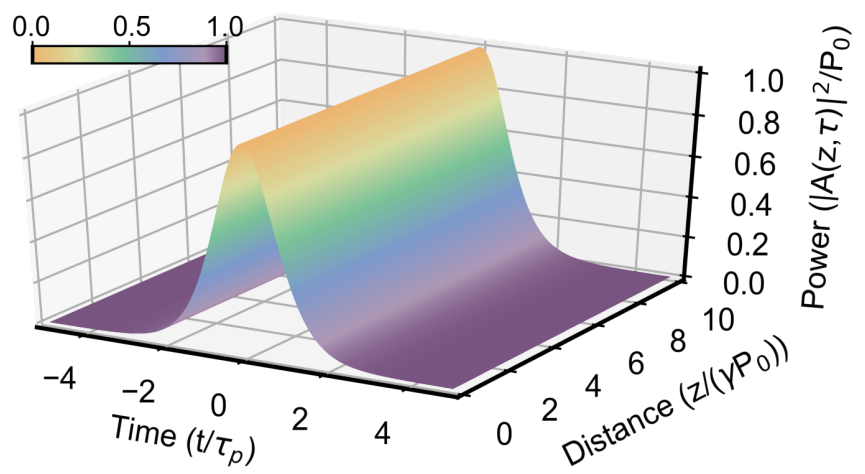

Fig. 2.7: Propagation of a fundamental soliton.

\subsection{Soliton Mode-Locking}

Laser oscillators are complex nonlinear systems that can support the formation of stable optical pulse trains. However, besides dispersion and SPM, also other effects need to be considered that contribute to the soliton-like pulse shaping (see figure 2.8). Upon a cavity roundtrip, the soliton is periodically perturbed by the action of gain, loss, bandwidth limiting and SAM, and stable pulse generation relies on a careful laser design to achieve the desired balance between the various effects. Their combined action on the laser field envelope is eventually summarized in the master equation of mode-locking in section 2.3.3.

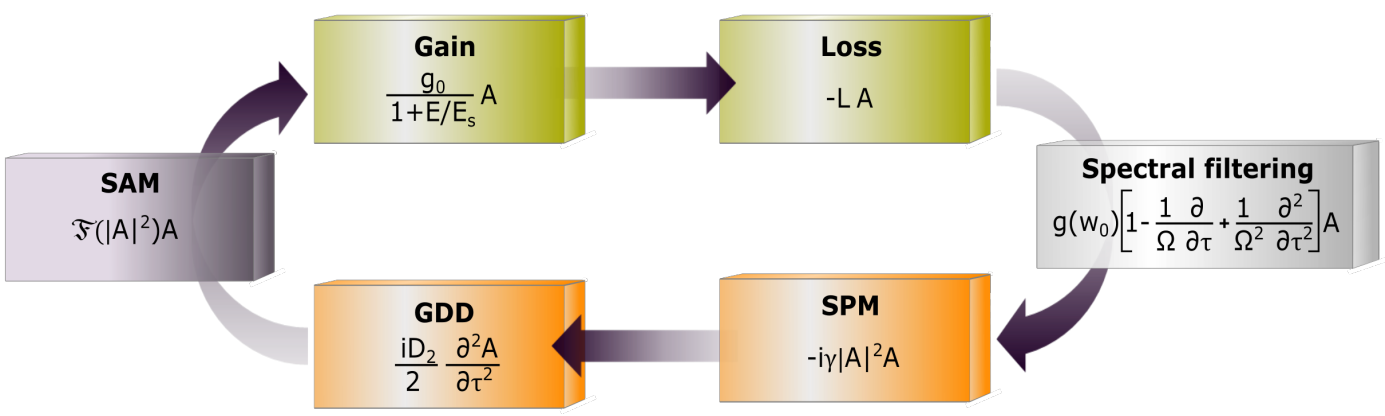

Fig. 2.8: Principal factors influencing the generation and evolution of ultrashort pulses. 


\subsubsection{Gain, Loss, and the Spectral Filtering}

The effect of loss is represented by a linear factor $l$ and also the saturable gain $g$ is assumed to have no effect on the pulse dynamics or shape [124, 135]:

$$
\frac{\partial A(z, \tau)}{\partial z}=(\underbrace{\frac{g_{0}}{1+E(z) / E_{s}}}_{g}-l) A(z, \tau) .
$$

Here, $g_{0}$ is the unsaturated (small signal) gain, $E(z)=\int|A(z, \tau)|^{2} d t$ is the laser pulse energy, and $E_{s}=\hbar w_{0} S / \sigma_{e m}$ is the gain saturation energy with the emission cross-section $\sigma_{e m}$ and the laser mode area $S$.

For solid-state lasers such as those based on $\mathrm{Cr}: \mathrm{ZnS} / \mathrm{ZnSe}$, the constant gain is a valid approximation since their upper-state lifetimes $\tau_{f}(\sim \mu s-m s)$ are typically much longer than the pulse repetition rates $(\sim n s)$, with $\sigma_{e m} \propto 1 / \tau_{f}$ for gaussian-shaped gain spectra [87]. In an intuitive picture, the long lifetime of the excited ions is associated with a high energy storage capacity of the gain medium, and only a sufficiently large number of photons that are passing by results in a reasonable chance to de-excite the ions back to the ground state. Hence, substantial gain saturation only occurs, when the time-integrated optical intensity, the so-called pulse fluence, approaches the saturation fluence $F_{s}=E_{s} / S$. In other words, only if $E / E_{s} \rightarrow 1$, dynamic gain saturation sets in. Here, the gain gets shaped by a pulse-induced and fast saturation, which recovers between consecutive pulses [136] This effect can be included by a time-dependent gain function $g(\tau)$, taking into account the characteristics of a four-level laser gain medium [137]:

$$
\frac{d g}{d \tau}=\sigma_{a b s}\left(g_{0}-g\right) \frac{I_{p}}{h \nu_{p}}-\frac{\sigma_{e m}}{h \nu} g|A|^{2}-\frac{g}{\tau_{g}} .
$$

Here, $\sigma_{a b s}$ is the absorption cross-section of the gain medium, $I_{p}$ and $\nu_{p}$ are the pump intensity and frequency, respectively, and $\tau_{g}$ is the characteristic time related to gain relaxation. In general, to obtain stable soliton mode-locking, the net saturated gain $g-l$ must be negative, otherwise the continuous-wave background radiation will also experience amplification.

Besides the effect of gain and loss, the finite amplification bandwith of the laser medium results in a spectral filtering action that curtails the pulse spectral bandwidth. Usually, a filter profile with a Lorentzian line shape and filter bandwidth $\Omega$ is assumed [138], which reads in the time domain as follows [124]:

$$
\frac{\partial A(z, \tau)}{\partial z} \approx g\left(w_{0}\right)\left[1-\frac{1}{\Omega} \frac{\partial}{\partial \tau}+\frac{1}{\Omega^{2}} \frac{\partial^{2}}{\partial \tau^{2}}\right] A(z, \tau) .
$$




\subsubsection{Self-Amplitude Modulation}

As discussed in section 2.1.2, KLM arises from an interplay between an intra-cavity aperture and beam self-focusing, in which intense pulsed operation is favored over CW lasing. This power discrimination effect is termed self-amplitude modulation and can be modelled as a saturable absorber-like action with a response function $\mathfrak{F}$ [124]:

$$
\begin{aligned}
\frac{\partial A(z, \tau)}{\partial z} & =\mathfrak{F}\left(|A(z, \tau)|^{2}\right) \cdot A(z, \tau), \\
\mathbf{H A}: \quad \mathfrak{F} & \approx-\frac{l^{\prime}}{1+\kappa|A|^{2}}, \\
\mathbf{S A}: \quad \mathfrak{F} & \approx-l^{\prime}+\kappa\left(1-\zeta|A|^{2}\right)|A|^{2},
\end{aligned}
$$

where the $\kappa$ - and $\zeta$ - parameters are the coefficients of self-amplitude modulation and alteration, respectively, and $l^{\prime}$ is the low-power saturable loss. The (loss) modulation functions of the saturable absorber depend on the intensity $I(z, \tau)=|A(z, \tau)|^{2}$, and hence they act instantaneously on the pulse.

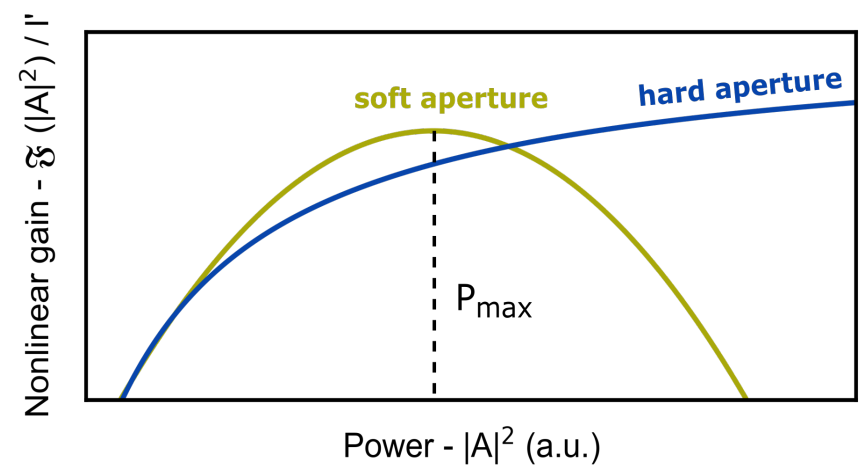

Fig. 2.9: Qualitative behaviour of the SAM functions $\mathfrak{F}\left(|A(z, \tau)|^{2}\right)$ for hard- and soft-aperture KLM.

For the case of hard-aperture (HA) KLM, this function can be described by a perfectly saturable absorber action, in which an increasing intra-cavity power reduces the beam size on a hard aperture. This self-focusing effect will eventually cause the diffraction loss $l^{\prime}$ to monotonically decrease, as shown in figure 2.9. However, if a soft-aperture (SA) is used, the behaviour changes: even though the loss is initially reduced by a growth in power, there is a turning point at which the loss gets enhanced again. The point of alteration is associated with a maximized overlapping between the pump and fundamental cavity beam in the gain medium. If the power is increased further, the self-focusing action will worsen the overlap and result in reduced gain. 


\subsubsection{The Master Equation of Mode-Locking}

The effects governing the pulse evolution in a laser cavity can be summarized by the master equation of mode-locking, that joins equations 2.10, 2.13, 2.17, 2.19 and 2.20 together [124]:

$$
\left.\frac{\partial A}{\partial z}=[g-l]+\frac{g\left(w_{0}\right)}{\Omega^{2}} \frac{\partial^{2}}{\partial \tau^{2}}+\stackrel{\text { Sain } / \text { loss }}{\left.\mathfrak{F}\left(|A|^{2}\right)\right]}\right] A+i\left[\frac{D_{2}}{2} \frac{\partial^{2}}{\partial \tau^{2}}-\frac{\text { SPM }}{\gamma|A|^{2}}\right] A,
$$

where it was assumed that only second-order dispersion $\left(D_{2}\right)$ is present. The equation was first introduced by Haus et al. [139] and provides a general description of the ultrashort pulses generated in solid-state [139] and fiber lasers [140]. It has secant-hyperbolic solutions of type [133, 139]:

$$
A(\tau)=A_{0} \cdot \operatorname{sech}\left(\frac{\tau}{\tau_{0}}\right) e^{\left(i \beta \cdot \log \left(\operatorname{sech}\left(\tau / \tau_{0}\right)\right)\right)}=A_{0} \cdot \operatorname{sech}^{1+i \beta}\left(\frac{\tau}{\tau_{0}}\right)
$$

with pulse duration $\tau_{0}$, chirp parameter $\beta$ and pulse amplitude $A_{0}$. The pulse duration and chirp depend on $D_{2}, \Omega, \gamma, \kappa$ and the pulse energy $E$ in a rather complicated fashion and are described in more detail in [133, 135, 139].
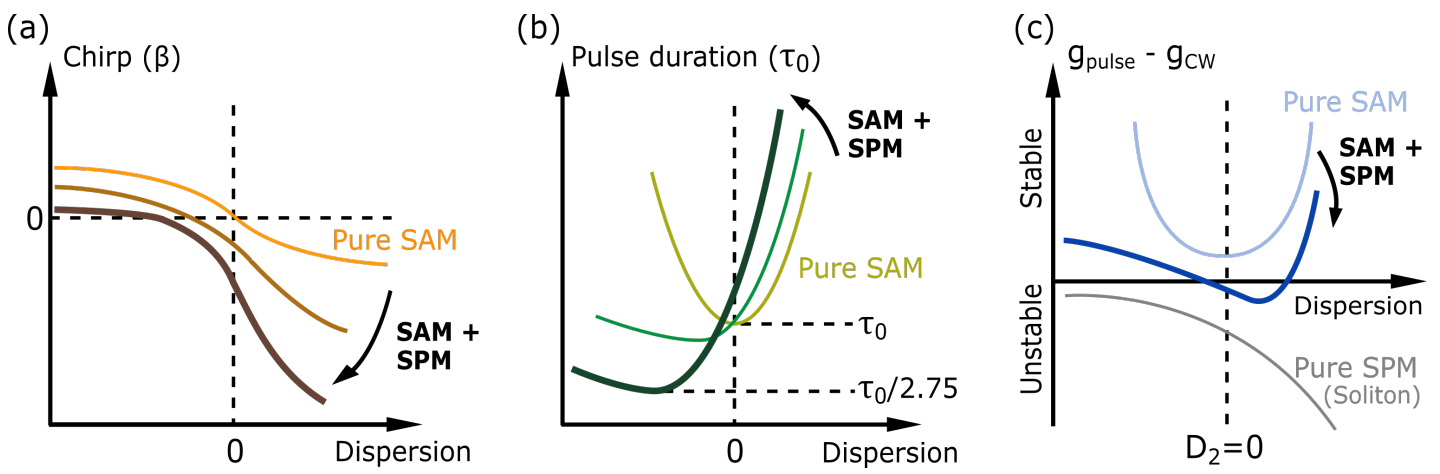

Fig. 2.10: Effect of dispersion $\left(D_{2}\right)$ and SPM on (a) the chirp, (b) the pulse width and (c) the stability for a fixed pulse energy and gain bandwidth but different values of SPM. As indicated in (c), only sufficient negative or positive dispersion can drive the system into stable operation. (The figure was adapted from [135]).

In figure 2.10, the qualitative behaviour of the chirp, the pulse width and the stability $\left(g_{\text {pulse }}-g_{\mathrm{cw}}\right)$ is plotted as a function of group velocity dispersion for different values of SPM $(\gamma)$. One can see that for an increasing amount of SPM, the point of minimum pulse duration is moved towards the negative dispersion regime. Moreover, when the effects of GVD and SPM are properly balanced, the pulse 
duration is reduced by a factor of 2.75 . Also, the solutions predicted by the master equation are not always stable and the competing CW oscillations can experience a greater gain $g_{\mathrm{cw}}$ than the laser pulses $g_{\text {pulse }}$. In this respect, a pure soliton laser without the effect of self-amplitude modulation is not stable against the CW background.

Interestingly, the start-up of mode-locked lasers can be captured in real-time via the use of time-stretch dispersive Fourier transform. In [141], a 20 fs KLM Ti:sapphire laser with a repetition rate of $90 \mathrm{MHz}$ was used to obtain a spectrally resolved view of the onset of femtosecond pulsing, evolving continuously from the initial picosecond fluctuations after several thousand cavity round-trips.

\subsubsection{The Effect of Different Dispersion Regimes}

Pulse formation, evolution and shaping is significantly affected by the intra-cavity dispersion and its interplay with nonlinear effects [142, 143]. In this section, two commonly applied mode-locking regimes are introduced that play an important role in various ultrafast solid-state and fiber laser systems.

\section{Dispersion-managed soliton formation [65]:}

So far, we have assumed the dispersion and self-phase modulation to be continuously distributed along pulse propagation. Strictly speaking, this is not true for typical femtosecond lasers. In the case of a solid-state bulk laser, for example, the discrete action of positive SPM and GDD in the laser crystal as well as the discrete action of negative dispersion introduced by chirped mirrors or material plates (see section 3.3.2) cannot be neglected anymore. In 1994, Spielmann et al. found that the precise arrangement of the cavity elements has a major impact on the pulse formation process, such that even the positioning of the output coupler plays a role in extracting the spectrally broadest pulses [144]. This knowledge has then enabled the generation of pulses with durations of less than two optical cycles ( $5 \mathrm{fs}$ ) in Ti:sapphire lasers [61].

As a general guideline for a solid-state laser, an equal distribution of negative dispersion into both arms of the cavity is beneficial and allows to exploit the KLM action twice per round-trip [95]. Moreover, when reaching shorter and shorter pulse durations, the dispersion swings between the negative and positive dispersion sections start to affect the pulse shape. While having a sech-shape at longer pulse durations, the pulse spectra start to get Gaussian-shaped and increasingly more flattopped for shorter pulse widths. 


\section{Chirped-pulse formation [124, 145]:}

Many applications benefit from both ultrashort and powerful laser systems. Scaling the intra-cavity peak power, however, unavoidably results in ever stronger nonlinearities in the gain medium, manifesting themselves in the fission of single coherent pulses to form chaotic multi-pulse emission. The saturable absorber action is then said to be "overdriven". In this respect, the regime of chirped-pulse formation can help to mitigate the limitations of classical soliton lasers operating in the net negative dispersion regime $\left(\mathrm{D}_{2}<0\right)$. As shown in figure 2.10 (c), highly chirped-pulse solutions accompanied by strongly increased pulse durations are found for $\mathrm{D}_{2}>0$ while the system is still running stably.

Whereas solitonic pulse-shaping due to a strict balance between SPM and negative GDD occurs for oscillators operating in the negative dispersion regime, the description of mode-locking in the positive dispersion regime is more challenging for the theoretical analysis. In [146], a chirped solitary pulse (CSP) solution was found analytically, revealing that the CSP has a Lorentzian spectral profile with truncations at some defined frequency $\Delta$ away from the carrier frequency. Even though the peak power can be kept below the instability threshold, the heavily chirped output pulses ( ps-range) of such laser systems need to be compressed externally to the few-cycle femtosecond regime again [147]. Also, because of the reduced pulse intensities, it might get difficult to initiate the pulse buildup process due to an insufficient saturation of the SAM.

\subsection{Chapter Summary}

In this chapter, the theoretical foundations behind ultrashort pulse generation have been laid, with a focus on soliton pulse propagation and soliton mode-locking. Starting with the concept of mode-locking in a simple Fabry-Pérot laser resonator, the advantageous technique of Kerr-lens mode-locking (KLM) was described and analyzed in detail, enabling the generation of few-cycle femtosecond pulses directly from a laser cavity. Conditions under which fundamental solitons are formed were also discussed on the basis of the nonlinear Schrödinger equation. Finally presented was the master equation of mode-locking which characterizes the complex interplay between the many effects governing the pulse evolution in a laser cavity, including self-amplitude and self-phase modulation. 


\section{THE FiRST Directly Diode-Pumped FEW-CyCle CR-Doped II-VI LASER}

In recent years, applications of mid-infrared radiation have proliferated tremendously, resulting in an ever-growing search for suitable laser sources that can directly provide the few-cycle pulses needed for efficient downstream MIR generation. To achieve the high sensitivity and specificity required for the spectroscopic investigation of (bio-)molecular samples in the MIR fingerprint region, sophisticated detection schemes such as electro-optic sampling (EOS) have to be combined with ultrafast laser technology to detect subtle changes encoded in the mid-infrared electro-magnetic waveform. However, the probe pulse duration in EOS-based measurements is a critical parameter since it limits the temporal resolution and thus the spectral bandwith that can be measured at high frequencies. Therefore, ultrashort probe pulses are highly sought after to increase the detection bandwidth. Moreover, if few-cycle laser pulses can be directly generated in the short-wave mid-infrared region at around 2-3 $\mu \mathrm{m}$, the conversion efficiency for frequency downconversion into the long-wavelength MIR beyond $3 \mu \mathrm{m}$ is significantly improved, while a broader selection of suitable nonlinear crystals is available also.

As outlined in chapter 1 , crystalline mid-infrared lasers based on $\mathrm{Cr}^{+2}: \mathrm{ZnS} / \mathrm{ZnSe}$ gain media are highly attractive candidates for directly providing coherent few-cycle radiation in the 2-3 $\mathrm{mm}$ range and - especially when combined with diode-pumping - offer an intrinsic low-noise performance and a much more compact alternative over commonly used systems. In this chapter, the first directly diode-pumped, Kerr-lens mode-locked $\mathrm{Cr}^{+2}$-doped II-VI oscillator, MIRanda1, will be presented, and a focus will be placed on the favourable material and spectroscopic properties of $\mathrm{Cr}^{+2}: \mathrm{ZnS} / \mathrm{ZnSe}$ (section 3.1) as well as on the major challenges associated with direct diode-pumping (section 3.2). Finally, the main findings will be summarized in section 3.4, highlighting the important breakthrough achieved with this laser system.

\subsection{Chromium-doped II-VI - The Titanium Sapphire of the Mid-Infrared}

Today's most popular femtosecond bulk lasers are certainly represented by the large number of reported and even commercially available Ti:sapphire $\left(\mathrm{Ti}^{3+} \mathrm{Al}_{2} \mathrm{O}_{3}\right)$ laser 
systems. These near-infrared lasers commonly deliver few-optical-cycle pulse durations and the recent advents of turn-key and diode-pumped versions have resulted in a diversification of application areas [148]. With transition-metal $\left(\mathrm{Cr}^{2+}\right)$-doped II-VI crystals (ZnS, ZnSe), a new class of laser materials was found in the 1990s to provide analogous properties in the 2-3 $\mu \mathrm{m}$ mid-IR spectral range [149-151], combining high emission cross-sections, fairly good mechanical and chemical stability as well as a similar thermal conductivity and ultrabroad bandwidth (see table 3.1).

(a)

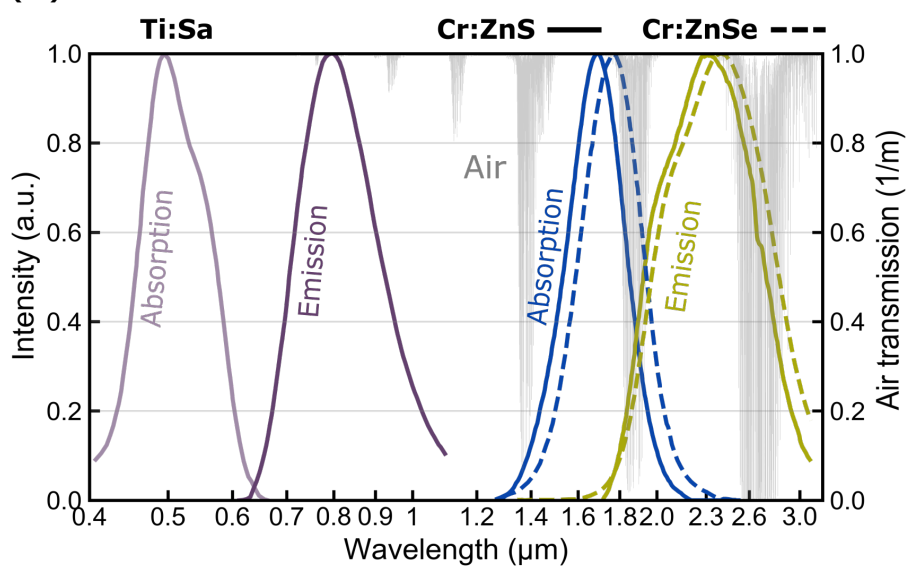

(b)

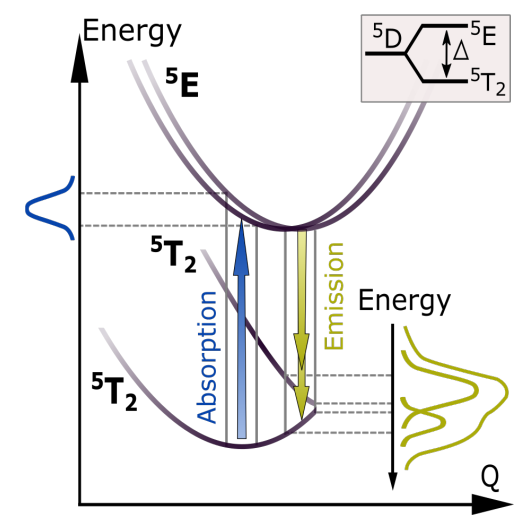

Fig. 3.1: (a) Absorption and emission spectra of Ti:sapphire and Cr:ZnS/ZnSe crystals together with the transmission of air obtained from HITRAN simulations. The wavelength axis is plotted on a logarithmic scale to preserve the relative bandwidth $\Delta \lambda / \lambda_{0}$. (b) Coordinational diagram of the laser transition in $\mathrm{Cr}: \mathrm{ZnS} / \mathrm{ZnSe}$, including the Jahn-Teller splitting. (Data taken from $[65,152])$.

Octahedrally coordinated $\mathrm{Ti}^{3+}$-doped $\mathrm{Al}_{2} \mathrm{O}_{3}$ and tetrahedrally coordinated $\mathrm{Cr}^{2+}$ doped ZnS/ZnSe are both crystals doped with transition metal (TM) ions. These kind of laser gain media exhibit a particularly strong interaction between the electronic states and lattice vibrations - the phonons [153, 154]. The vibrationalelectronic ("vibronic") interaction is associated with a pronounced homogeneous line broadening of the laser transition and results in very broad absorption and emission bands. It gives rise to a rather complicated energy band level scheme which can hardly be described by the simple three- or four-level picture ${ }^{1}$. In fact, vibronic line broadening also takes place in prominent rare-earth ions such as trivalent lanthanides $\left(\mathrm{Tm}^{3+}, \mathrm{Ho}^{3+}, \mathrm{Er}^{3+}\right)[67]$, which also provide radiation in the MIR spectral range.

1 The first ruby laser, for example, belongs to the class of three-level lasers and the lasing transition happens between two specific electronic states. These sharp electronic transitions result in a very narrow-linewidth laser emission. 


\subsection{Chromium-doped II-VI - The Titanium Sapphire of the Mid-Infrared 33}

The interaction with the lattice crystalline field is, however, much weaker than for TM-ions.

In the case of $\mathrm{Cr}: \mathrm{ZnS} / \mathrm{ZnSe}$, a broad absorption band centered at around 1.6-1.8 $\mu \mathrm{m}$ corresponds to the transition between the ${ }^{5} \mathrm{~T}_{2}$ ground state and the ${ }^{5} \mathrm{E}$ excited state, and is the only spin-allowed transition for this system [65, 155]. In addition, both involved levels are split due to the Jahn-Teller effect, which is stronger in the ground state (see figure 3.1). Therefore, the absorption spectrum is single-peaked, whereas the fluorescence gets double-peaked, with $\mathrm{Cr}^{2+}$-doped materials exhibiting one of the largest relative bandwidths $\Delta \lambda / \lambda_{0}$ of more than 0.35 . Also, given the fundamental scaling rule for the required pump intensity at threshold, $I_{t h} \propto\left(\Delta \lambda / \lambda_{0}\right) \lambda_{0}^{-4}[67]$, the longer infrared wavelengths compared to Ti:sapphire are of additional benefit.

\begin{tabular}{lccc}
\hline & $\mathbf{C r}^{2+}: \mathbf{Z n S}$ & $\mathbf{C r}^{2+}: \mathbf{Z n S e}$ & $\mathbf{T i}^{3+}: \mathbf{A l}_{2} \mathbf{O}_{3}$ \\
\hline$\Delta \lambda / \lambda_{0}$ & 0.38 & 0.37 & 0.3 \\
$\lambda_{0}$ & 2350 & 2450 & 790 \\
$\Delta \lambda$ & 890 & 900 & 230 \\
$\tau$ & 4.3 & 5.5 & 3.2 \\
$\sigma_{\text {abs }}$ & 100 & 110 & 6.5 \\
$\sigma_{\text {em }}$ & 140 & 130 & 39 \\
$\kappa$ & 27 & 18 & 27 \\
dn/dT & 46 & 70 & 12 \\
Hardness (Knoop) & 160 & 120 & 2000 \\
Bandgap & 3.8 & 2.8 & 6 \\
Ref. & {$[155-157]$} & {$[67,155,157]$} & {$[157-159]$} \\
\hline
\end{tabular}

Table 3.1: Material and spectroscopic characteristics of the broadband laser materials $\mathrm{Cr}^{2+}: \mathrm{ZnS} / \mathrm{ZnSe}$ and $\mathrm{Ti}^{3+}$ :Sapphire. $\Delta \lambda / \lambda_{0}$ : relative emission bandwidth; $\lambda_{0}(\mathrm{~nm})$ : maximum gain wavelength; $\Delta \lambda(\mathrm{nm})$ : gain bandwidth (FWHM); $\tau$ ( $\mu \mathrm{s})$ : fluorescence lifetime; $\sigma_{\mathrm{abs}}\left(10^{-20} \mathrm{~cm}^{2}\right)$ : peak absorption cross-section; $\sigma_{\mathrm{em}}\left(10^{-20} \mathrm{~cm}^{2}\right)$ : peak emission cross-section; $\kappa(\mathrm{W} /(\mathrm{m} \cdot \mathrm{K}))$ : thermal conductivity; $\mathrm{dn} / \mathrm{dT}\left(10^{-6} 1 /{ }^{\circ} \mathrm{C}\right)$ : thermo-optic coefficient; bandgap energy $(\mathrm{eV})$. All parameters refer to operation at room temperature $(\sim 300 \mathrm{~K})$.

Typically, mid-IR vibronic laser materials are characterized by high nonradiative decay rates at room temperature [67]. The onset of strong multiphonon relaxation processes leads to a pronounced decline of their upper-state lifetimes and therefore decreases ("quenches") the upper-level population. As a consequence, the gain efficiency will be drastically reduced, requiring cryogenic cooling. $\mathrm{Cr}^{2+}$-doped $\mathrm{ZnS} / \mathrm{ZnSe}$, however, can be conveniently operated at room temperature [160]. The fluorescence lifetime measurements of $\mathrm{Cr}: \mathrm{ZnS}$ reveal a constant lifetime well beyond $300 \mathrm{~K}$, while the one of $\mathrm{Cr}: \mathrm{ZnS}$ shortens by $20 \%$ at $300 \mathrm{~K}$ - similar to Ti:sapphire. 
Since zinc-blende II-VI semiconductor compounds like Cr:ZnS/ZnSe do not possess inversion symmetry and have a high nonlinear index of refraction (see table 2.2) - even 30-times higher than that of sapphire — strong second- and third-order nonlinearity are expected $[64,155]$. In this respect, polycrystalline $\mathbf{C r}: \mathbf{Z n S} / \mathbf{Z n S e}$ crystals play a special role as they were also used in the three diode-pumped systems presented in this thesis. Hampered by sublimation during the growth process and suffering from the resulting poor uniformity of single-crystal Cr:ZnS/ZnSe, the simpler production of polycrystalline material has relaxed the requirements on crystal quality while attracting growing interest in recent years [161]. The latter is certainly driven by the appearance of random quasi-phase-matching (RQPM) [162]: because the average grain size within the polycrystalline medium is on the order of the coherence length of typical nonlinear optical processes, efficient generation of higher-order harmonics such as the second harmonic ( $\mathrm{SH}$ ) were already demonstrated [64, 163, 164].

In 2009, the first Kerr-lens mode-locked operation of a $\mathrm{Cr}^{2+}: \mathrm{ZnS} / \mathrm{ZnSe}$ laser was realized $[165,166]$, and since then ever shorter output pulse durations were achieved - finally rendering their broadband and ultrafast performance as ideal starting point to exploit further nonlinear frequency conversion $[30,33,73,167]$. As will be demonstrated in the last experimental chapter 5.3 , the $2-3 \mu \mathrm{m}$ output radiation can be efficiently converted through intra-pulse difference frequency generation (IDFG) into the deeper MIR range (here: $4.5-14 \mu \mathrm{m}$ ) in a relatively straightforward and simple arrangement.

\subsection{Challenges of Diode-Pumping}

With their ultrabroad absorption bands, Cr:ZnS/ZnSe lasers offer great flexibility in terms of pump wavelength selection. Ideally, the crystals are pumped close to their respective absorption peaks near $1.695 \mu \mathrm{m}(\mathrm{Cr}: \mathrm{ZnS})$ and $1.770 \mu \mathrm{m}$ (Cr:ZnSe), but only very few CW laser sources exist that can provide sufficient pump power in this spectral range. Over the past years, a large variety of different pump laser sources was successfully implemented, including rare-earth doped fiber and solid-state lasers (Er ${ }^{3+} @ 1.6 \mu \mathrm{m}[156,163,168], \mathrm{Tm}^{3+} @ 1.9 \mu \mathrm{m}$ [169-171], $\mathrm{Ho}^{3+}$ @2.1 um [172]), Raman-shifted $\mathrm{Nd}^{3+}$ lasers (@1.6 $\left.\mu \mathrm{m}[173,174]\right), \mathrm{NaCl}: \mathrm{OH}$ color

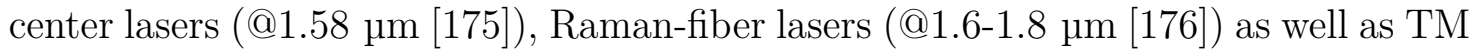
doped solid-state lasers ( $\left.\mathrm{Co}: \mathrm{MgF}_{2} @ 1.86 \mu \mathrm{m}[150]\right)$. To date, virtually all ultrafast $\mathrm{Cr}: \mathrm{ZnS} / \mathrm{ZnSe}$ lasers are pumped by mature and high-power Er or Tm fiber lasers, mainly thanks to their near diffraction-limited output beam characteristics and ease of use. Consequently, up to 2 watts of average power could already be demonstrated with Kerr-lens mode-locked oscillators [177]. 
On the other hand, the low pump threshold of Cr:ZnS/ZnSe gain crystals allows for direct diode-pumping and represents an excellent alternative that combines the low-noise performance, compactness, high-efficiency, and affordability needed for laser-based applications. Therefore, bringing cutting-edge laser diode technology and few-cycle 2-3 $\mu \mathrm{m}$ solid-state lasers together will help meet the growing demand for accessible and reliable table-top mid-IR sources [178]. The first diode-pumped operation of Cr:ZnS/ZnSe oscillators was demonstrated in 1997 in a side-pumped configuration $^{2}$ [179], while a longitudinally pumped continuous-wave cavity was realized only a few years later [170]. When using single-emitter laser diodes, output powers of more than $400 \mathrm{~mW}$ could be achieved from the Cr:ZnS/ZnSe lasers so far [180], and a record output power of even $3.7 \mathrm{~W}$ was demonstrated when the gain crystal was pumped by a $30 \mathrm{~W}$ diode bar [181]. Finally, the first femtosecond operation of a diode-pumped $\mathrm{Cr}^{2+}$-doped II-VI oscillator was realized in 2012 [182], resulting in average output powers of $50 \mathrm{~mW}$. By using a $3.5 \mathrm{~W}$ single-emitter laser diode at $1550 \mathrm{~nm}$ as well as a SESAM device to allow for self-started mode-locking (see section 2.1.2), a pulse duration of 180 fs was achieved, which was significantly longer than what is supported by the gain medium's bandwidth.

On the contrary, the technique of Kerr-lens mode-locking (see section 2.1.2) is known to support much shorter pulse durations [183], which can even exceed the emission bandwidth-limit of the gain medium [184, 185]. Since it relies on the action of a Kerr lens inside the laser crystal, a good mode-matching between pump and fundamental cavity mode is crucial to obtain sufficient gain and a good power discrimination between CW and pulsed operation. Selecting the most suitable laser diode type is therefore of utmost importance.

\subsubsection{The Right Choice of Laser Diode Type}

Laser diodes are available in a myriad of different designs and output beam characteristics. To choose the right type, one can start with a set of parameters defined by the corresponding application. For this thesis, the desired characteristics are summarized in table 3.2, indicating the need for a laser diode beam with the highest possible beam quality to ensure good focusability. Even though single-mode laser diodes with $M^{2} \approx 1$ exist, their output power is limited to a few $100 \mathrm{~mW}$ only. This is a main drawback of laser diodes because a higher beam quality usually comes along with a very much reduced output power. Therefore, multi-mode laser diodes need to be used, and a good compromise between output power and beam quality can be provided by single-emitter laser diodes (see section 3.2.2). However, if

\footnotetext{
2 The output of this laser cannot be considered as truly continuous-wave, given that the laser diode array was operated in a pulsed mode ( $50 \mu$ s pulsewidth).
} 
several single-emitter diodes are stacked up vertically to increase the total output power in the form of a laser diode bar, the brightness will deteriorate dramatically. The resulting poor beam quality is reflected by the high $M^{2}$ values of $>100$ or even $>1000$ in both directions. For comparison, a diffraction-limited Gaussian beam is characterized by an $M^{2}$ factor of 1 . Hence, using diode bars for pumping ultrafast KLM oscillators is not an option.

\begin{tabular}{ll}
\hline Parameter & Desired value \\
\hline Output power & Several watts \\
Beam quality & Ideally single-mode, $M^{2}=1$ \\
Wavelength & $\lambda=1.6-1.8 \mu \mathrm{m}$ \\
Wavelength stability & $\Delta \lambda<10 \mathrm{~nm}$ \\
Beam delivery & Free space, not fiber-coupled \\
\hline
\end{tabular}

Table 3.2: Desired output characteristics of a laser diode to be used for the few-cycle oscillators presented in this thesis.

In terms of output wavelength, semiconductor laser diodes based on indium phosphide (InP) are the most promising candidates to provide lasing beyond $1.6 \mu \mathrm{m}$ [186]. Single-frequency operation is not specifically demanded since the absorption band of $\mathrm{Cr}: \mathrm{ZnS} / \mathrm{ZnSe}$ crystals is broadband enough to sustain variations of about $\Delta \lambda<10 \mathrm{~nm}$ without a significant drop in gain. Finally, multi-mode fiber-coupled packaging should be avoided to maintain the high unidirectional beam quality provided by free-space single-emitter diodes. Putting all these desired characteristics together, C-Mount packaged single-emitter laser diodes provide an ideal solution and were used for the three laser systems presented in the remaining part of this thesis.

\subsubsection{Properties of Single-Emitter Diodes}

Semiconductor laser diodes are typically operated in a forward-biased double-hetero p-i-n structure, in which an undoped junction acts as the laser-active layer $[86,187]$. Because of its higher refractive index and reflective ends, light gets tightly confined and amplified inside this region when an external voltage is applied.

\section{Output beam properties:}

The active layer of the utilized single-emitter diodes has a rectangular shaped cross section with a horizontal ("slow axis") and vertical ("fast axis") dimension of $95 \mu \mathrm{m}$ and $1 \mu \mathrm{m}$, respectively. Compared to true single-mode diodes with an emitter width 
of only a few microns, the increased volume allows to generate and extract more laser power. However, it comes at the expense of a reduced brightness in the slow axis direction, where multiple transverse modes are emitted in a closely spaced manner [188] (see figure 3.2(b)). Typical beam quality factors are $M^{2} \simeq 1$ (fast) and $M^{2} \gg 1$ (slow). The strong diffraction at the output facet results in a highly elliptic and divergent emission, with the TE modes gradually merging together in the horizontal direction to form a light line. Depending on the number and intensity distribution of the mixed modes, the diode emission can have a variety of different shapes, and a pronounced dependance on the applied pump current was found experimentally (see figure 3.3).

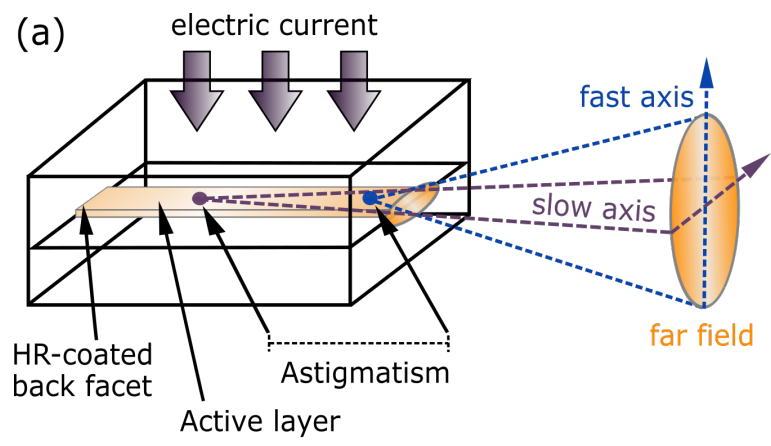

(b)

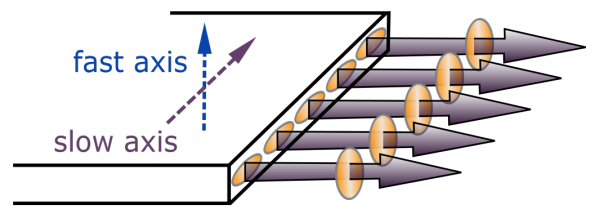

(c)
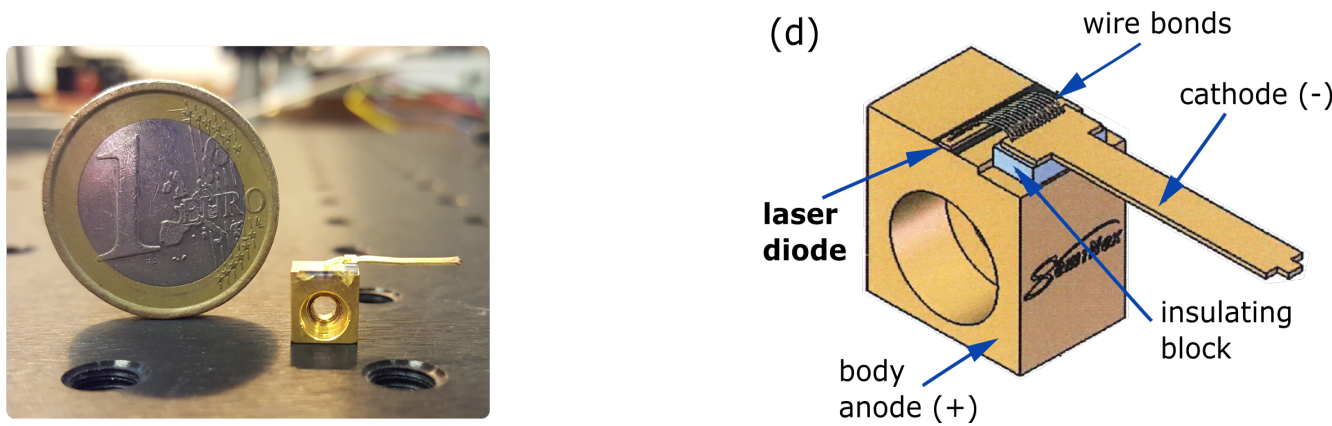

Fig. 3.2: (a) The anistropic output emission of a laser diode. For the sake of clarity, the degree of astigmatism is much exaggerated. (b) The output emission of a wide stripe laser diode containing multiple TE modes in the slow axis (horizontal) direction. (c) Picture of a C-Mount diode next to a $1 €$-coin to illustrate the small dimensions. (d) Components of a C-Mount diode (figure provided by SemiNex Corp.).

Even though wide stripe laser diodes can now deliver up to a few watts of average power, temperature-related problems have significantly diminished the maximum output of diodes emitting around 1.5-1.7 $\mu \mathrm{m}$ [189-191]. But, since the output of laser diodes is linearly polarized along the slow axis direction, polarization multiplexing schemes can be used to increase the total effective power (see section 4.1.1). 
(a)

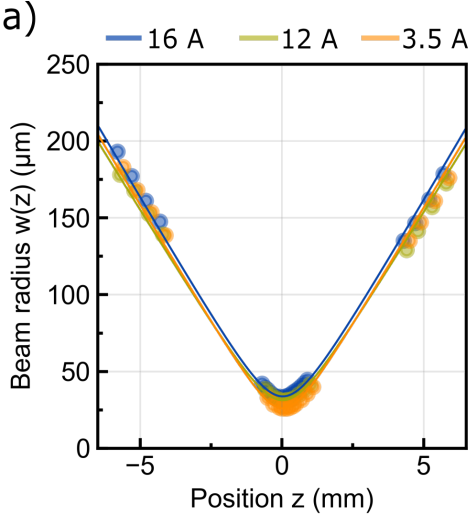

(b)

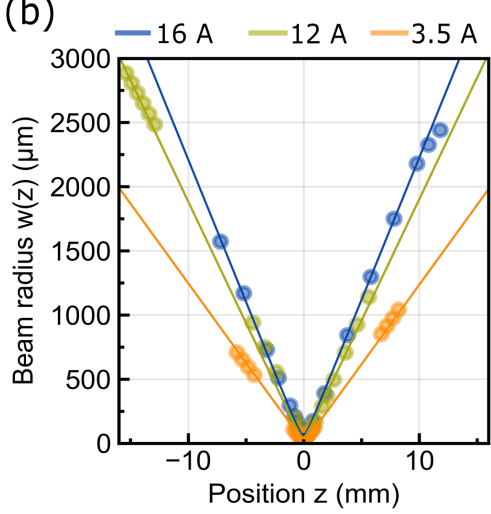

(c)

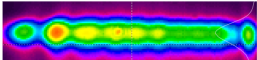

$17 \mathrm{~A}$
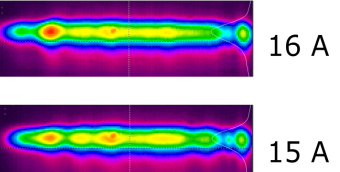

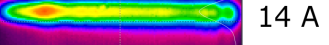

Fig. 3.3: Beam quality and beam profile measurements of the laser diode output for different driving currents. (a) Results for the fast axis, with calculated $M^{2}$-values of 1.5 (3.5 A), 1.8 (12 A) and $2.1(16 \mathrm{~A})$. (b) Results for the slow axis, with calculated $M^{2}$-values of $9.1(3.5 \mathrm{~A}), 17$ $(12 \mathrm{~A})$ and $28(16 \mathrm{~A})$. In (c), the output beam was collimated and measured with a pyroelectric camera (Spiricon Ophir Photonics, $P Y-I I I-C-B$ ) and reveals spatial changes in the beam profile for rather small steps in pump current.

\section{Noise properties:}

The amplitude noise of most solid-state and semiconductor lasers is dominated by a characteristic relaxation oscillation (RO), which only appears when the upper state lifetime $\tau_{2}$ is substantially longer ${ }^{3}$ than the laser cavity decay time $\tau_{c}$ $[86,88]$. Inititally, when these lasers are suddenly switched on, the laser output power may exhibit several spikes due to fluctuations in the population inversion and laser gain that will settle down fairly quickly into a quasi-sinusoidal decaying oscillation about the steady-state value. After a given amount of time and in the case of a very quiet and stable environment, the output intensity can eventually be treated as constant. However, when the laser is perturbed by any sudden change in gain, pump power, cavity alignment or cavity loss, another transient relaxation oscillation will be triggered and will again evolve in a exponentially decaying manner.

Cr:ZnS/ZnSe as well as rare-earth doped fiber lasers are both solid-state laser sources that are prone to relaxation oscillations. In addition, the noise performance of a Cr:ZnS/ZnSe laser system is greatly affected by the noise of its pump source since pump noise can be transferred to oscillator noise below the oscillator's relaxation oscillation frequency $\nu_{r o}[192]$. The latter can be written as [86, 88]:

$$
\nu_{r o}=\frac{1}{2 \pi} \sqrt{(x-1) /\left(\tau_{2} \tau_{c}\right)},
$$

\footnotetext{
3 This condition does not hold for most gas lasers, so that spiking and relaxation oscillations are in general not observed.
} 
where $\tau_{c}=-\nu_{\text {rep }}^{-1} \log \left[R_{1} R_{2}\left(1-T_{i}\right)^{2}\right]$ and $x=P_{\text {pump }} / P_{\text {th }}$ with the pump power $P_{\text {pump }}$ and the threshold power $P_{t h}$.

(a)

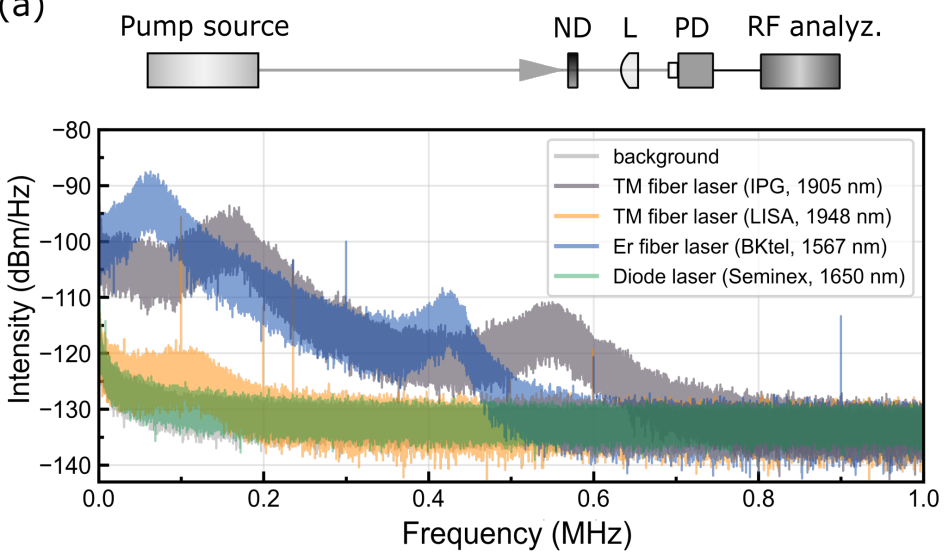

(b)

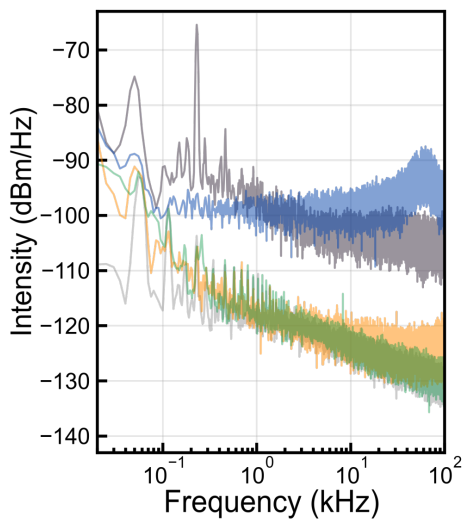

Fig. 3.4: Qualitative comparison of the amplitude noise of available pump sources for Cr:ZnS/ZnSe oscillators. The measurements reveal the strong relaxation oscillations in the kHz-range, which are subject to standard rare-earth doped TM (IPG, TLR-120-WC-Y12) and Er fiber lasers (Bktel, HPFL-370-1567-FCAPC). But, fiber lasers can also be designed for lower noise operation (for example: Tm laser, LISA laser products, 101030 010). By contrast, semiconductor laser diodes (here: SemiNex Corp., C-132) reveal an intrinsic low noise performance, which is very close to the performed background noise measurement taken without any incoming signal. For the measurements, the pump light was focused by a lens (L) onto a biased InGaAs photodetector (PD, Thorlabs DET10D2), which is sensitive from 0.9-2.6 $\mu \mathrm{m}$. The DC voltage generated by the PD was kept constant for all measurements with the aid of a neutral density (ND) filter wheel, and the amplitude noise signal was measured by a high dynamic-range radio-frequeny (RF) spectrum analyzer (Agilent, E4447A) in a $1 \mathrm{MHz}$-range (see (a)). (b) The low-frequency noise is plotted on a logarithmic scale for frequencies up to $100 \mathrm{kHz}$.

Let us assume that we have set up a Cr:ZnS laser with a repetition rate of $\nu_{\text {rep }}=50 \mathrm{MHz}$, an end-mirror reflectivity of $R_{1}=1$, an output coupler reflectivity of $R_{2}=0.75$, and an internal loss per pass of $T_{i}=0.05$. Then, $\tau_{c} \approx 7.8 \mathrm{~ns}$ and together with $\tau_{2}=4.3$ us (see table 3.1 ), the relaxation oscillation frequency will amount to $\nu_{r o, C r: Z n S} \approx \mathbf{8 7 0} \mathbf{k H z}$ for a pump rate of $x=2$. Rare-earth doped fiber lasers, which are the most common pump source of Cr:ZnS/ZnSe lasers, have RO frequencies that lie in the few $10 \mathrm{kHz}$ to a few $100 \mathrm{kHz}$ range [193-196]. Therefore, their noise characteristics will be transferred and added to the Cr:ZnS laser noise if they are not suppressed by sophisticated feedback schemes [197].

By contrast, semiconductor laser diodes also exhibit relaxation oscillations but in a very different frequency domain [86, 88]. A GaAs laser diode, for example, with a cavity length of $\mathrm{L}=300 \mu \mathrm{m}$, a pump rate of $x=1.5$, a cavity round-trip time of $\tau_{\text {rep }} \approx 6.7 \mathrm{ps}$ and lifetime $\tau_{c} \approx 1.1 \mathrm{ps}$, and an upper state lifetime in the p-n 
junction of $\tau_{2} \approx 3 \mathrm{~ns}$, has a RO frequency ${ }^{4}$ of about $\nu_{r o, G a A s} \approx \mathbf{2} \mathbf{G H z}$. This means that the relaxation oscillations of laser diodes are located in the multiple $\mathrm{GHz}$-range and thus experience stronger damping by the $\mathrm{Cr}^{2+}$-doped $\mathrm{ZnS} / \mathrm{ZnSe}$ gain medium because $\nu_{r o, G a A s} \gg \nu_{r o, C r: Z n S}$.

In figure 3.4, the amplitude noise measurement of a laser diode is compared to the results obtained with three different rare-earth doped fiber lasers, which have been available at our research laboratory. The noise signals were recorded in a $1 \mathrm{MHz}-\mathrm{span}$ in the baseband region and clearly reveal the intrinsic low-noise characteristics of a laser diode. This is of great interest for mid-infrared applications such as near-field scanning optical microscopy (s-SNOM, PF-SNOM) [15, 198], which typically operate at tapping frequencies in the $\mathrm{kHz}$-domain and are highly sensitive to noise in this range.

\subsubsection{Effects on the Laser System and the Few-Cycle Pulse Generation}

The lower brightness of laser diodes requires a series of precautions when designing and setting up a few-cycle laser system, especially when the technique of softaperture Kerr-lens mode-locking is used. Compared to fiber laser-pumped systems, some major challenges arise that will be discussed in the following.

\subsubsection{Mode-Matching Considerations}

Adapting the pumped volume of the crystal to the volume occupied by the fundamental laser resonator mode is known as mode-matching and plays a crucial role when selecting lenses for pump beam collimation. The optimization of the overlap is not only important for extracting the highest possible gain and thus output power of the cavity, but also to exploit efficient self-amplitude modulation for KLM (see section 2.3.2). Dealing with the highly anisotropic and non-Gaussian like pump beam of a single-emitter laser diode is a demanding task since the brightness of the fast and slow axis emission differs significantly and greatly affects the pumped crystal volume. For optimized mode-matching, a set of aspheric and/or cylindrical lenses is therefore needed to reshape and collimate each axis in a separate manner.

In literature, several guidelines have been reported on improving a laser system's performance by an appropriate choice of pump and laser beam diameter [199-205]. However, most of them cannot directly be applied to the cases considered in this thesis. For example, when assuming a perfectly uniform pump distribution of radius

\footnotetext{
4 Note that the condition $\tau_{2} \gg \tau_{c}$ is met in all considered cases.
} 
$w_{p}$ and a Gaussian-shaped resonator mode of 1/e-radius $w$, both are ideally chosen to be equal in size inside the gain crystal $\left(w_{p} / w \simeq 1\right)[199]$. While this can be readily fulfilled with single-mode pump sources, it does not hold for the multi-transversemode emission of a single-emitter laser diode. Also, most theoretical investigations already start with a given pump beam size/ellipticity and aim to find the most suitable mode size for the laser cavity to be built [200, 203, 204]. Consequently, the laser cavity mode is also allowed to be of elliptic shape, which can be realized through cylindrical cavity optics [201, 202]. Building a laser cavity around a given pump setup is, yet, not practical for the laser systems considered in this thesis, in which the laser mode sizes are already defined by the envisaged resonator geometry.

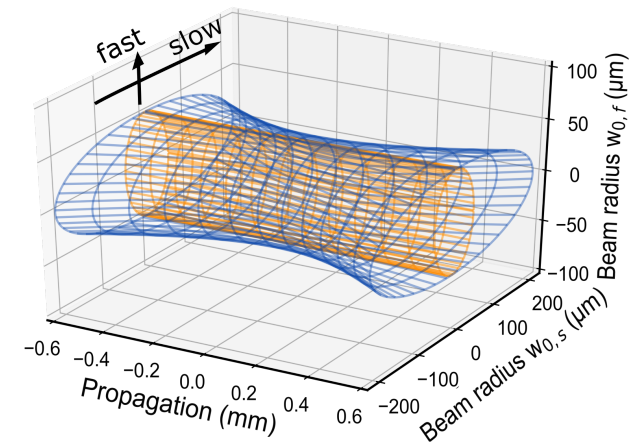

Fig. 3.5: Simulation of the overlapped volume between the laser diode beam (blue) and the fundamental cavity beam (orange) along the pump absorption length.

\begin{tabular}{l|cc}
$\mathbf{M}_{\mathbf{f}, \mathbf{s}}^{\mathbf{2}}=\mathbf{1 . 2} / \mathbf{7 . 6}$ & $\mathbf{w}_{\mathbf{0}, \mathbf{s}} / \mathrm{w}_{\mathbf{0}, \mathbf{f}}$ & Overlap \\
\hline $\mathrm{w}_{\mathbf{0}, \mathbf{f}}=30 \mu \mathrm{m}$ & $\mathbf{3 . 2}$ & $22 \%$ \\
$\mathrm{w}_{\mathbf{0}, \mathbf{f}}=50 \mu \mathrm{m}$ & $\mathbf{1 . 9}$ & $36 \%$ \\
$\mathrm{w}_{\mathbf{0}, \mathbf{f}}=55 \mu \mathrm{m}$ & $\mathbf{1 . 8}$ & $39 \%$ \\
\hline & & \\
$\mathbf{M}_{\mathbf{f}, \mathbf{s}}^{\mathbf{2}}=\mathbf{1 . 5} / \mathbf{9}$ & & \\
\hline $\mathrm{w}_{\mathbf{0}, \mathbf{f}}=30 \mu \mathrm{m}$ & $\mathbf{3 . 9}$ & $17 \%$ \\
$\mathrm{w}_{\mathbf{0}, \mathbf{f}}=50 \mu \mathrm{m}$ & $\mathbf{2 . 3}$ & $30 \%$ \\
$\mathrm{w}_{\mathbf{0}, \mathbf{f}}=55 \mu \mathrm{m}$ & $\mathbf{2 . 1}$ & $33 \%$
\end{tabular}

Table 3.3: Best simulation results for given $M^{2}$-values of the pump beam and focus size $\mathrm{w}_{0, f}$ for the fast axis. The overlap is given with respect to the pumped volume $( \pm 0.5 L)$.

Nevertheless, a good approximation can be obtained when considering the overlap volume between a perfect Gaussian cavity beam $\left(M^{2}=1\right)$ and an elliptic pump beam. Even though it is a simplified model and does not take thermal and spatial effects like self-focusing or gain saturation into account, it provides a general idea of the optimal diameter ratio between the fast and slow axis of the pump laser diode. A useful guidance is provided in [201], in which the pump absorption length $L$ is taken as a measure to optimize the pump beam and cavity mode diameters. After the propagation length $L$, namely, the intensity of an incident light beam has already decayed to $1 / \mathrm{e}(\approx 37 \%)$ of its initial value and the intensity after a certain distance can be written as [81]:

$$
I(z)=I_{0} \cdot e^{-z / L}=I_{0} \cdot e^{-N_{d o p} \sigma_{a b s} z},
$$

where $N_{d o p}$ and $\sigma_{a b s}$ are the crystal's doping concentration and absorption cross section, respectively. In this chapter, a $5 \mathrm{~mm}$-long Cr:ZnSe crystal with $N_{\text {dop }}=$ $7.5 \cdot 10^{18} \mathrm{~cm}^{-3}$ and $\sigma_{a b s}=110 \cdot 10^{-20} \mathrm{~cm}^{2}$ (see table 3.1) was used, resulting in a 
pump absorption length of $L \approx 1.2 \mathrm{~mm}$. Since the fast axis emission of the diode is of near diffraction-limited beam quality $\left(M^{2} \approx 1\right)$, its waist radius $w_{0, f}$ in the focus was set equal to the mode waist radius $w_{0}$ of the fundamental cavity beam. This, we believe, is essential for efficient soft-aperture KLM (see also reference [206]). In table 3.3, the simulation results are depicted for different waist sizes $w_{0, f}$, which are chosen to resemble the three diode-pumped laser systems of this thesis.

The simulations predict that an axis ratio of about $2-2.5$ in the focus might be ideal to achieve the highest gain, which also agrees well with the ratios reported for diode-pumped KLM Ti:sapphire systems [206-208]. In general, keeping the slow axis diameter as confined as possible also minimizes the risk to excite other than the fundamental cavity mode.

(a)

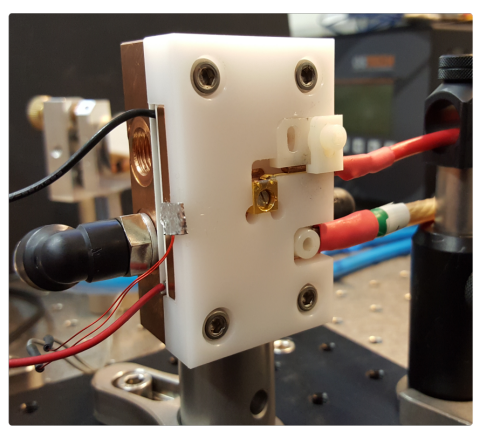

(b)

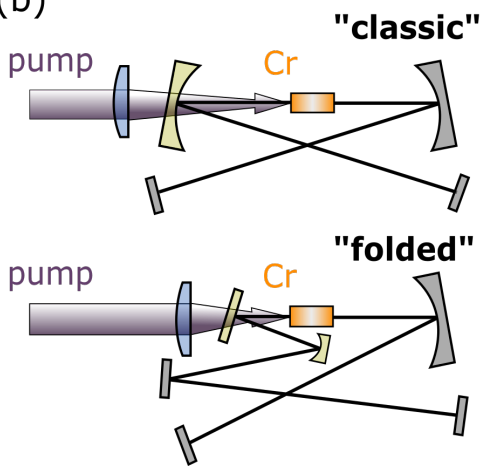

(c)

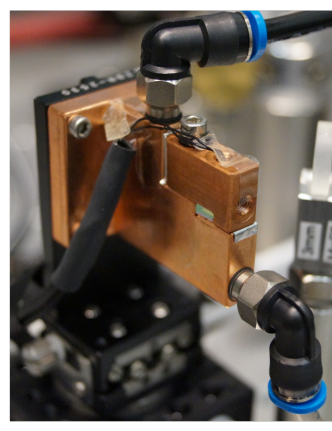

Fig. 3.6: (a) Picture of the laser diode mount, which was equipped with a peltier element. (b) Schematic sketch of a "classic" and "folded" bow-tie cavity. (c) The $5 \mathrm{~mm}$ long gain crystal (clear aperture: $2 \times 2 \mathrm{~mm}$ ) was placed inside a water-cooled copper mount.

\subsubsection{Technical Challenges}

Apart from a proper selection of aspherical and cylindrical lenses to shape the diode output beam, some technical — but not insignificant — challenges arise that need to be considered in the laser design:

- Pump beam optics: A spot size ratio of about 2:1 requires the collimated slow axis diameter to be much larger in size than the fast axis diameter before being focused into the laser crystal. In fact, it was found experimentally that the slow axis diameter had to be close to 1 inch $(25.4 \mathrm{~mm})$ in size, which is the standard size for commonly available optics. Therefore, customized rectangular-shaped mirrors were used and highly-reflective (HR) coated for the pump wavelength. 
- Pump diode handling: The laser diodes had been delivered without any heatsink or mount attached to it. To ensure an adequate heat removal for the waste heat produced and to avoid damage by electrostatic discharge, a special mount had to be designed (see figure 3.6 (a)). Since a typical InP single-emitter diode delivers up to 4 watts of output power at $15 \mathrm{~A} / 1.8 \mathrm{~V}$, only $15 \%$ of electricity get converted into light. The waste heat of $23 \mathrm{~W}$ is generated within a small area of only $7 \times 6 \mathrm{~mm}$ defined by the C-mount body. In the first design, water-cooled components as well as a Peltier element were implemented to stabilize the diode's output power via thermoelectric cooling.

- Plane input coupler mirror: A standard four-mirror cavity consists of two flat end-mirrors and two curved mirrors with the gain crystal placed in between (see figure 3.6 (b)). The pump beam is then focused by a suitable lens through one of the curved mirrors. This "classic" bow-tie design is, however, hard to realize for diode-pumped systems, since it limits the selection of suitable pump lenses needed to achieve the required tight focus in the gain crystal. For practical reasons, a "folded" bow-tie cavity design had to be implemented in the first two laser systems ${ }^{5}$. In this case, the pump beam enters the cavity through a plane input coupler mirror, and pump focusing optics with a focal length equal or shorter than the focal length of the cavitie's curved mirrors can be used.

- Crystal dimensions: The low brightness of the slow axis causes the pump beam to diverge rapidly when focused to a tiny spot. To avoid clipping at the crystal end facets, a gain medium of suitable dimension is required. The Cr:ZnS/ZnSe gain media available in our lab are typically $5 \mathrm{~mm}$ and $9 \mathrm{~mm}$ thick. Therefore, taking the measured $M^{2}$-value of about 7.6 for the slow axis and the corresponding pump spot diameter of about $90 \mu \mathrm{m}$, which is true for the first laser system built, a slow axis radius of $w( \pm 2.5 \mathrm{~mm})=0.7 \mathrm{~mm}$ and $w( \pm 4.5 \mathrm{~mm})=1.25 \mathrm{~mm}$ is calculated at the crystal end facets ${ }^{6}$. Therefore, crystals with clear apertures of at least $2-3 \mathrm{~mm}$ are needed (see figure 3.6 (c)).

- Cavity alignment: To build and align the laser oscillator, the pump beam typically serves as guidance. Using the large collimated pump beam of a wide stripe laser diode is, however, not practical, especially when it diverges rapidly after passing through a focal plane. In this thesis, a solution was found by either relying on the single-mode emission of an Erbium fiber laser $(\lambda=1567 \mathrm{~nm})$, which was temporarily installed as an alternative pump source,

\footnotetext{
5 For the third laser system, a workaround could be found based on a novel pump focusing scheme (see chapter 5.2.1)

6 This assumes that the waist is directly positioned in the center of the crystal.
} 
or by using a red alignment laser diode. The latter has the advantage to provide radiation visible to the human eye, therefore facilitating the setup of the cavity.

\subsection{Few-Cycle Pulse Generation via Kerr-Lens Mode-Locking}

In the following, the results of the first directly diode-pumped $\mathrm{KLM} \mathrm{Cr}^{2+}$-doped IIVI laser are presented along with a focus on the intra-cavity dispersion management, the success of the soft-aperture Kerr-lens mode-locking technique, and the measured amplitude noise performance. Even though this first demonstration has already been published in reference [209], the following subsections serve to provide a deeper insight into the experimental observations and the results achieved.

\subsubsection{Pump Setup and Resonator Design}

A single indium phosphide (InP)-based C-Mount laser diode (SemiNex Corp., C-132) was used, providing up to $3.8 \mathrm{~W}$ at $1650 \mathrm{~nm}$ from a $95 \mu \mathrm{m}$ wide stripe. To stabilize the diode's output power and wavelength and mitigate the thermal power roll-off, which is characteristic for these laser diodes [190], simultaneous thermoelectric and water cooling was implemented. The measured output power is shown in figure 3.7 (a), clearly indicating the unavoidable onset of the roll-off at higher pump current levels. To evaluate the output beam quality in the horizontal and vertical direction, the $\mathrm{D}_{4 \sigma}$ beam radii were measured with the help of a scanning slit profiler (Ophir, NanoScan 2s) at different positions along the optical axis z. Following the ISO standard ${ }^{7} 11146$ [210], the measured beam radii $w(z)$ were related to the $M^{2}$-factor, the wavelength $\lambda$, and the measured radius at the beam waist, $w_{0}=w\left(z=z_{0}\right)$, according to [211]:

$$
w(z)^{2}=w_{0}^{2}\left[1+\left(z-z_{0}\right)^{2}\left(\frac{M^{2} \lambda}{\pi w_{0}^{2}}\right)^{2}\right] .
$$

A fit through the data points revealed $M^{2}$-values of 1.2 and 7.6 for the fast and slow axis of the diode, respectively (see figure 3.7 (b)). Based on the modematching considerations and the technical challenges listed in the previous section, the anisotropic and highly-divergent output of the diode was then reshaped with a set of AR-coated aspheric and cylindrical lenses.

\footnotetext{
7 It demands that half of the measured data points must be located at a distance greater than two Rayleigh lengths away from the beam focus, whereas the other half of the points is measured around the focused beam waist.
} 
(a)

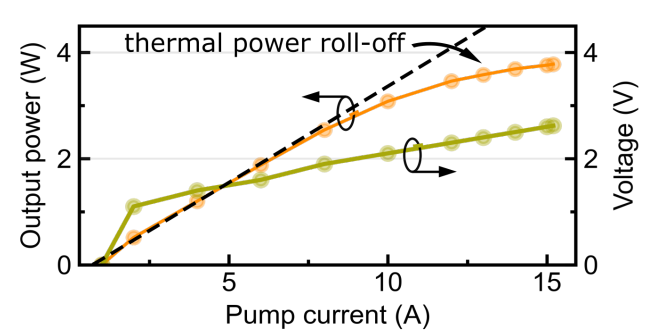

(b)

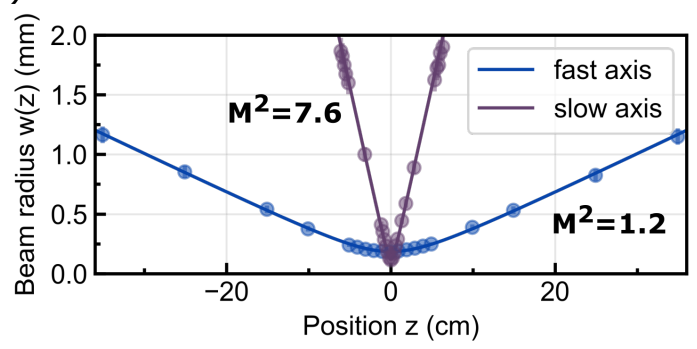

Fig. 3.7: (a) Laser diode output power and voltage versus pump current. (b) $M^{2}$-measurement of the fast (vertical) and slow (horizontal) axis emission. Note that this data was taken at a rather low pump current (2-3 A) since the change in beam quality for increasing pump currents was not yet known at that point.

(a)

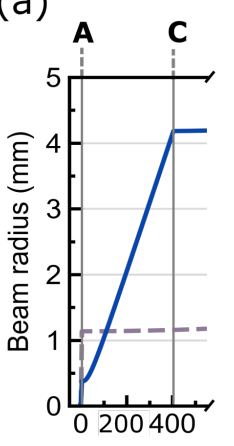

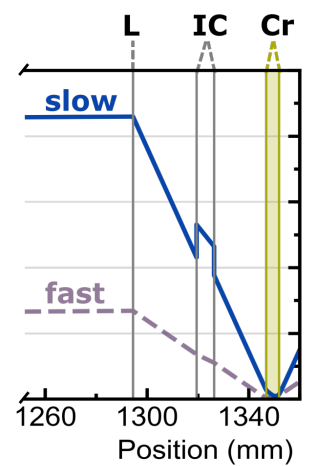

Cr:ZnSe

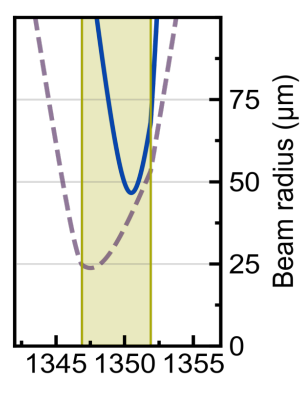

(b)

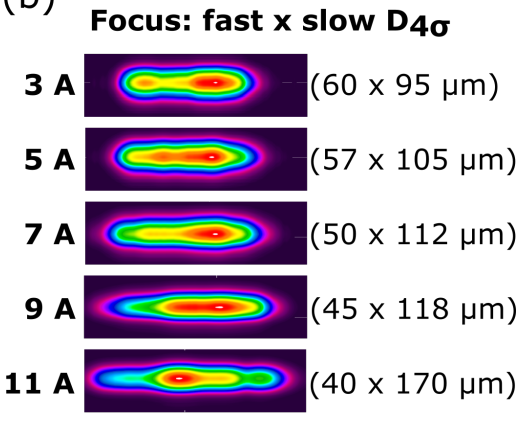

Fig. 3.8: (a) Simulated pump beam propagation for the fast and slow axis using the open source software reZonator. To shape the output emission, an aspheric $(\mathrm{A}, \mathrm{f}=4.51 \mathrm{~mm})$ and cylindrical lens $(\mathrm{C}, \mathrm{f}=400 \mathrm{~mm})$ were used, with the beam being focused by an aspheric pump lens $(\mathrm{L}, \mathrm{f}=50 \mathrm{~mm})$ trough a $6 \mathrm{~mm}$ thick plane-plane input coupler mirror (IC) placed at $45^{\circ}$. The green shaded area marks the focal area inside the gain crystal. (b) Change in pump spot diameters for increasing current levels.

To identify the most suitable lens combination for a desired pump spot size ratio of $\sim 1: 2$ (fast:slow axis) and a theoretical cavity mode diameter of $\sim 60 \mu \mathrm{m}$ in the focus, ABCD-matrix calculations had been performed, with the final result being depicted in figure 3.8 (a). Taking into account the measured beam divergences and radii, it predicts a theoretical pump spot diameter of $48 \mu \mathrm{m}$ (fast) x $94 \mu \mathrm{m}$ (slow) and is in good agreement with the measured spot diameter of approximately $60 \mu \mathrm{m}$ x $95 \mathrm{\mu m}$. The latter, however, is only true for a very modest pump current of $3 \mathrm{~A}$ (see figure $3.8(\mathrm{~b})$ ). In fact, later measurements revealed that this deviation becomes larger for increasing pump currents. The particulary strong change in pump beam characteristics beyond 11 A manifested itself in a drop in continuous-wave oscillator output power, when pump currents larger than 11 A were applied. At that point, 
corresponding thermal problems related to the ever increasing heat load inside the crystal could be excluded by monitoring the crystal's surface temperature via a NTC sensor. The latter has been attached to the copper mount of the gain medium.

(a)

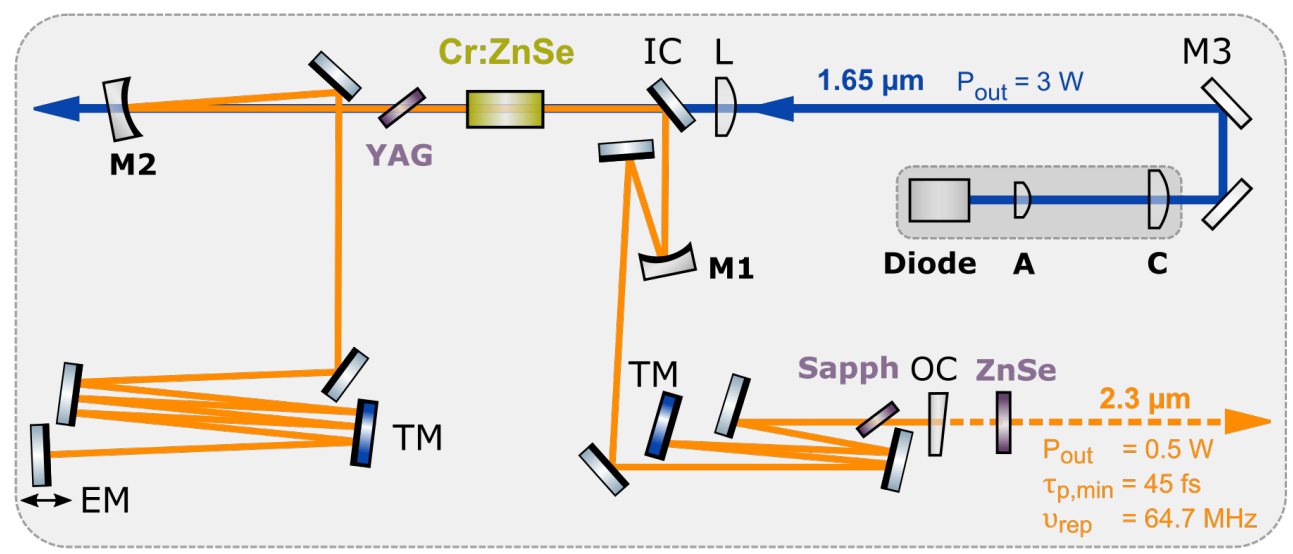

(b)

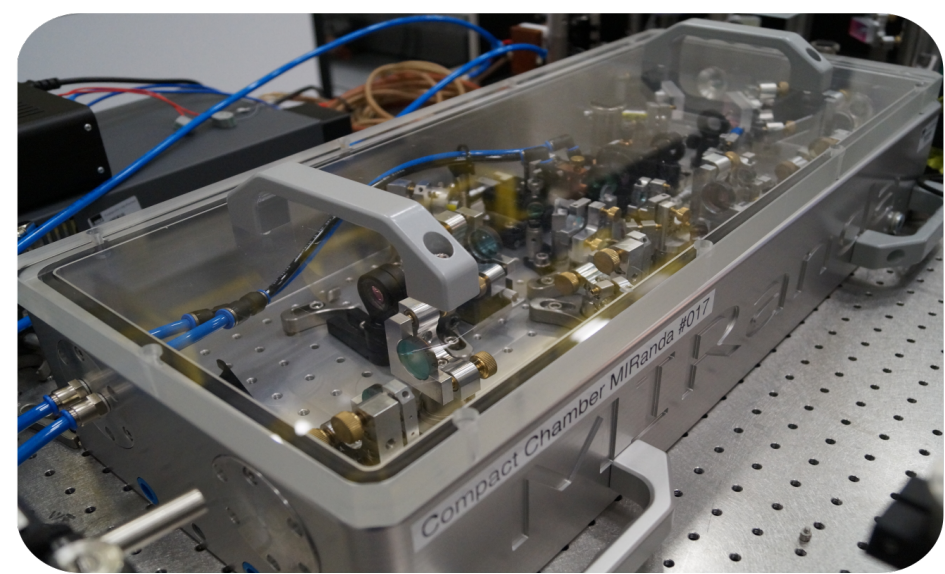

Fig. 3.9: (a) Schematic of the experimental setup of MIRanda1 and (b) a picture of the real laser system in the lab. M1 and M2: curved mirrors $\left(\mathrm{ROC}_{\mathrm{M} 1}=-100 \mathrm{~mm}\right.$, $\left.\mathrm{ROC}_{\mathrm{M} 2}=-200 \mathrm{~mm}\right)$, M3: highly-reflective rectangular-shaped mirrors for the pump laser $(1.4 \mu \mathrm{m}-1.7 \mu \mathrm{m}), \mathrm{A}$ : aspheric lens $(\mathrm{f}=4.51 \mathrm{~mm})$, C: cylindrical lens $(\mathrm{f}=400 \mathrm{~mm})$, L: aspheric pump lens $(\mathrm{f}=50 \mathrm{~mm}), \mathrm{Cr}: \mathrm{ZnSe}$ : gain medium $(5 \mathrm{~mm})$, Brewster-oriented material (3 mm YAG - for astigmatism compensation, $3 \mathrm{~mm}$ Sapphire - for dispersion compensation), OC: output coupler (34\%), TM: third-order dispersive mirror (blueshaded), ZnSe: $5 \mathrm{~mm}$ AR-coated substrate to temporally re-compress the output pulses. Non-labelled mirrors, including the input coupler (IC) and the end-mirror (EM), are HR-coated (2.0-2.6 $\mu \mathrm{m})$ and transmit the pump light.

Figure 3.9 shows a schematic of the experimental setup along with a picture of the real system in the laboratory environment. The oscillator contained a $5 \mathrm{~mm}$ long 
anti-reflection (AR)-coated Cr:ZnSe polycrystalline gain element (IPG Photonics) with a clear aperture of $2 \cdot 2 \mathrm{~mm}$ and a doping concentration of $7.5 \cdot 10^{18} \mathrm{~cm}^{-3}$. It was mounted inside a water-cooled copper heat $\operatorname{sink}\left(\mathrm{T}=12^{\circ} \mathrm{C}\right)$ and the single-pass absorption at $1650 \mathrm{~nm}$ was measured to be $\sim 90 \%$. Compared to a gain medium placed at Brewster's angle, in which a strong beam expansion in the tangential (horizontal) plane arises from a refraction at the material's surfaces [212, 213], an AR-coated crystal allows for a more homogeneous spot size while reducing asymmetric thermal lensing effects [177] (see figure 3.10) ${ }^{8}$. Moreover, when using an AR-coated crystal, the pump beam does not need to be p-polarized for minimal reflection losses and can theoretically take arbitrary polarization directions. This results from the fact that $\mathrm{Cr}: \mathrm{ZnS} / \mathrm{ZnSe}$ crystals do not exhibit any considerable polarization dependence with regard to their absorption cross-sections [214].

(a)
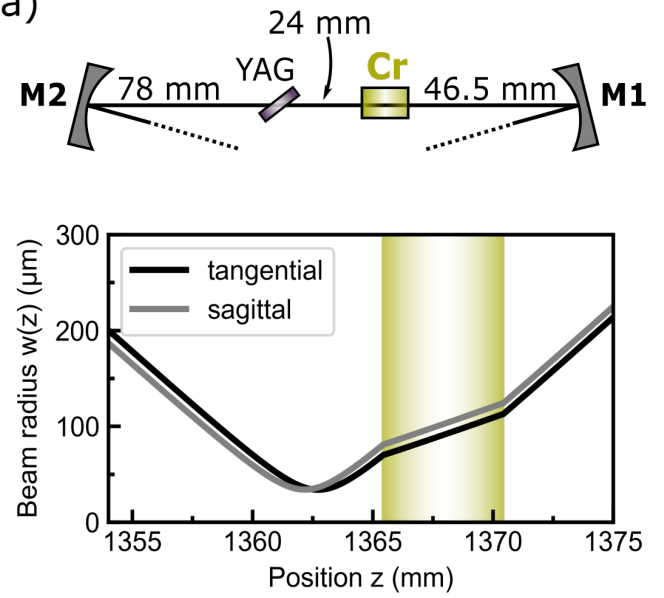

(b)
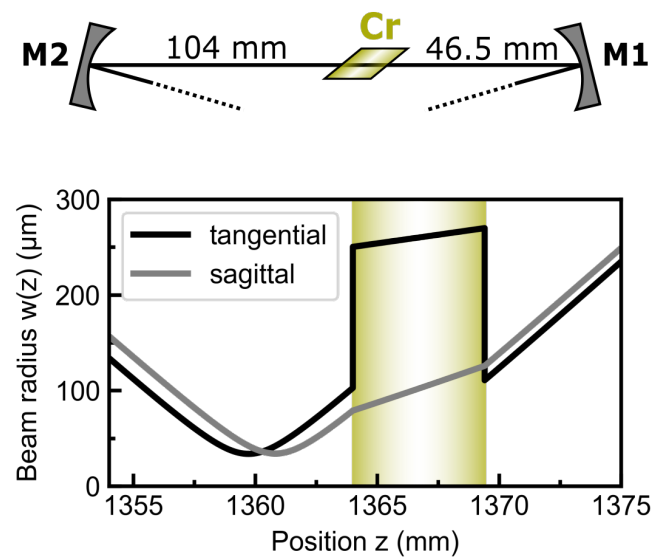

Fig. 3.10: Illustration of the difference in beam radii for (a) an AR-coated crystal and (b) a crystal placed at Brewster's angle. For the ABCD-matrix calculations, similar parameters were used, however, an additional $3 \mathrm{~mm}$ YAG-plate was placed inside the cavity of figure (a) to compensate for astigmatic distortions caused by an oblique angle of incidence on the curved mirrors.

As illustrated in figure 3.9, the Cr:ZnSe crystal was placed between two curved mirrors M1 and M2 inside an asymmetric X-fold cavity with an arm length ratio of $\overline{\mathrm{M} 1-\mathrm{OC}} / \overline{\mathrm{M} 2-\mathrm{EM}}=0.7$. This is in accordance with the KLM design procedure suggested in reference [215], in which a reasonable trade-off between stable laser operation and high modulation efficiency can be achieved for arm ratios between $2 / 3$ and $3 / 4$. Since the selection of suitable optics was limited at the time of the oscillator construction, the radii of curvature (ROC) for M1 and M2 were different,

\footnotetext{
8 Note that the pronounced jump in the tangential beam size - as it is depicted in figure 3.10 (b) - is not an exact representation of the real beam distortions at the crystal's surfaces.
} 
but chosen to increase the focal spot diameter in the Cr:ZnSe crystal. With this, the nonlinear effects in the gain crystal are reduced and the onset of multi-pulsing for increasing pump powers can be prevented [216]. In addition, astigmatic distortions are introduced due to the oblique angle of incidence on the curved mirrors, and a $3 \mathrm{~mm}$-thick Brewster plate (YAG) is placed beside the gain medium to avoid a corresponding degradation of the laser performance. Using multiple highly-reflective 45-degree mirrors inside the laser oscillator allowed to assemble the whole cavity - excluding the pump setup — inside a monolithic housing with a footprint of only $30 \mathrm{~cm}$ by $70 \mathrm{~cm}$.

\subsubsection{The Importance of Intra-cavity Dispersion Management}

Even though the ultrabroad emission cross-section of $\mathrm{Cr}^{2+}$-doped II-VI gain crystals provides an ideal condition for the direct generation of few-cycle laser light, the pulse duration of KLM laser systems will ultimately be limited by the degree of higher-order dispersion control inside the oscillator. As explained in the theoretical section 2.2, the self-phase modulation arising from the Kerr effect in the gain crystal needs to be balanced by an appropriate amount of negative GDD, such that solitonic pulse formation can appear in ultrafast solid-state laser systems. In the first diode-pumped Cr:ZnSe laser, material plates made of sapphire were incorporated into the cavity. As listed in table 2.2 of section 2.2.1, this material is able to provide suitable negative GVD, which can also account for the large positive second-order dispersion of the gain crystal. For generating the shortest pulses, however, a small but constant total GDD per round-trip is required over the entire spectral range [217], with the pulse duration of the soliton scaling as $\tau_{0} \propto \sqrt{\left|D_{2}\right|}$ for a given value $D_{2}<0$ of the intra-cavity GDD (see theory chapter 2.2.3). Therefore, the additional third-order dispersion accumulated by the gain crystal and added material plates needs to be addressed as well when operation close to zero dispersion is desired. In this respect, the design and usage of tailored dispersive mirrors (DM) has become quite popular.

Introduced in 1994 [218], laser mirrors based on chirped multilayer coatings turned out to be indispensible for the few-cycle pulse generation and octave-spanning spectra demonstrated with Ti:sapphire laser systems [61, 219]. When designed carefully, these mirrors uniquely combine ultra-high reflectivity (typically $>99.5 \%$ ) with well controlled dispersion characteristics over a broad spectral operation range [220]. The frequency-dependent chirp caused upon mirror reflection arises from the varying thickness of the multiple layers, such that different wavelengths penetrate different path lengths into the stack (see inset of figure 3.11 (b)). Beside this chirped mirror $(\mathrm{CM})$ technology, there is also the principle of resonant storage, in which nanoscale 
Fabry-Pérot-like cavities are embedded into the multilayer structure to resonantly trap selected wavelengths $[221,222]$. The combination of wavelength-dependent penetration and resonance effects is realized in modern dispersive mirrors to provide high reflectivity and GDD over a broad wavelength range without increasing the thickness of the coating. Recently, this technology has also become available for ultrafast 2-3 $\mathrm{mm} \mathrm{Cr}: \mathrm{ZnS} / \mathrm{ZnSe}$-laser systems [223] and was directly applied to the laser systems presented in this thesis.

(a)

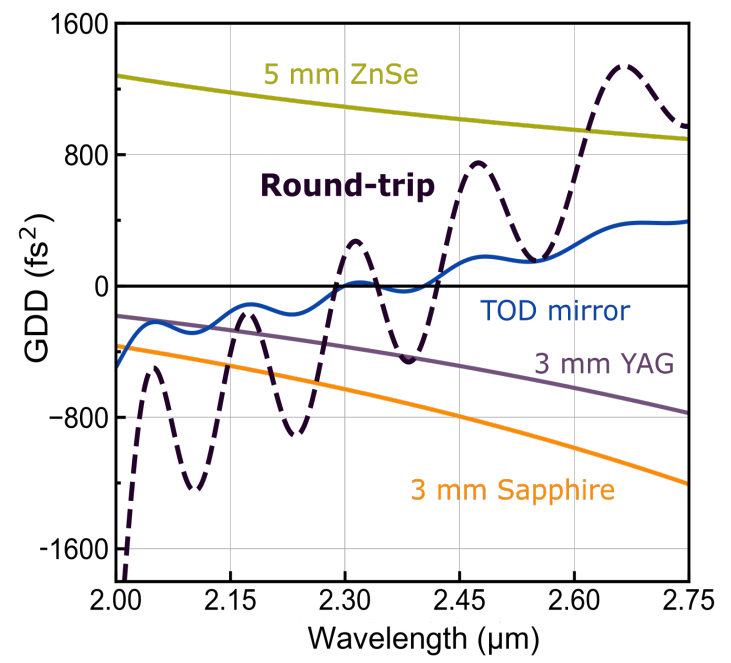

(b)

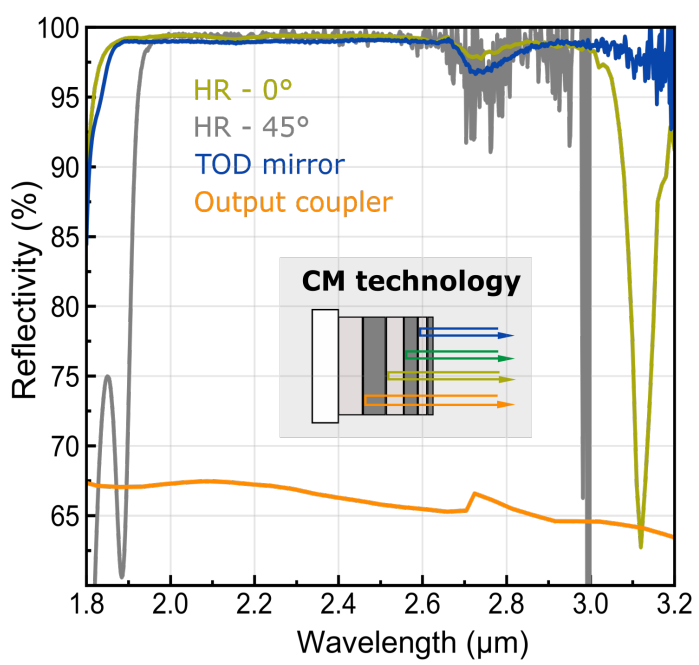

Fig. 3.11: (a) Theoretical dispersion values of intra-cavity elements including the single-pass GDD of incorporated material plates as well as the dispersion introduced by a single TOD mirror. The calculated theoretical round-trip dispersion for the case of eight total bounces on the TOD mirror is shown as a dashed line. (b) Measured reflectivity curves (p-polarized) of the oscillator mirrors that were designed to operate in a broadband spectral range $(2.0-2.6 \mu \mathrm{m})$.

In figure 3.11, the reflectivity and second-order dispersion of various intra-cavity elements, including ZnSe, YAG, sapphire and dispersive mirrors, are depicted. The latter was designed to only compensate for the materials' third-order dispersion (TOD) without adding additional GDD at the central wavelength $(\lambda \sim 2.3 \mu \mathrm{m})$. Also plotted is the calculated theoretical round-trip dispersion for the combination of mirrors and material plates that experimentally resulted in the shortest pulse duration. However, it is important to point out that the exact dispersion values were not experimentally measured, and each individual dispersive mirror may exhibit a slightly different oscillation pattern.

For efficient Kerr-lens mode-locking, laser operation close to the limits of one of the oscillator's stability ranges is required [101, 215, 224]. The stability zones for MIRanda1 are shown in figure 3.12 (b), and were calculated by evaluating the 
stability parameter of the ABCD round-trip cavity matrix [88, 225] for a varying distance $L$ between the curved mirror $M 2$ and the YAG plate. Following the guidelines on hard-aperture KLM presented in [215], the laser was adjusted to operate closer to the 2 nd edge of stability zone I $(L 1=78 \mathrm{~mm})$ with the Cr:ZnSe crystal being slightly shifted towards mirror $M 1$ of the shorter resonator arm (see figure 3.12 (a)). Stable soft-aperture KLM operation could then be initiated by slightly mis-aligning and rapidly moving ${ }^{9}$ the end-mirror (EM) that was mounted on a mechanical translation stage. Because of the polycrystalline nature of the gain medium and the effect of random quasi-phase-matching (see section 3.1), the onset of second-harmonic light was a clear indicator for mode-locked operation, when the gain crystal's surface was monitored by an infrared-sensitive camera. In addition, the theoretical beam radii for $\mathrm{CW}$ and pulsed operation have been calculated and are depicted in figure 3.12 (b).

(a)
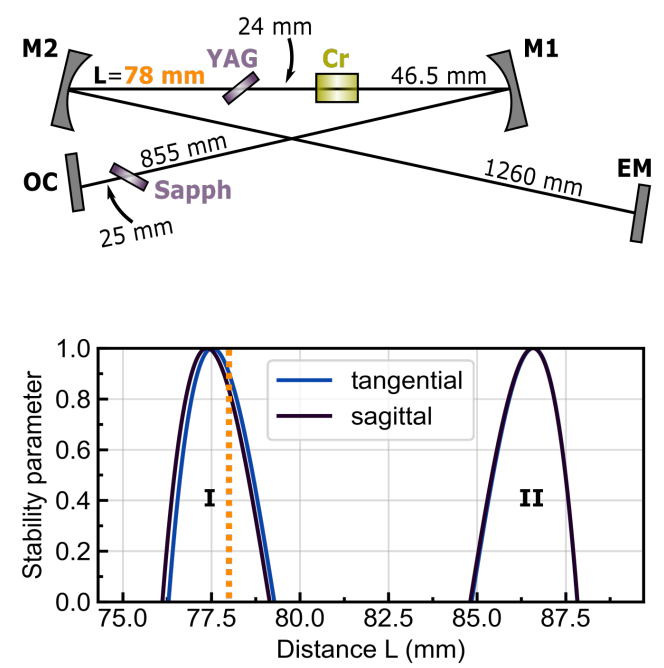

(b)

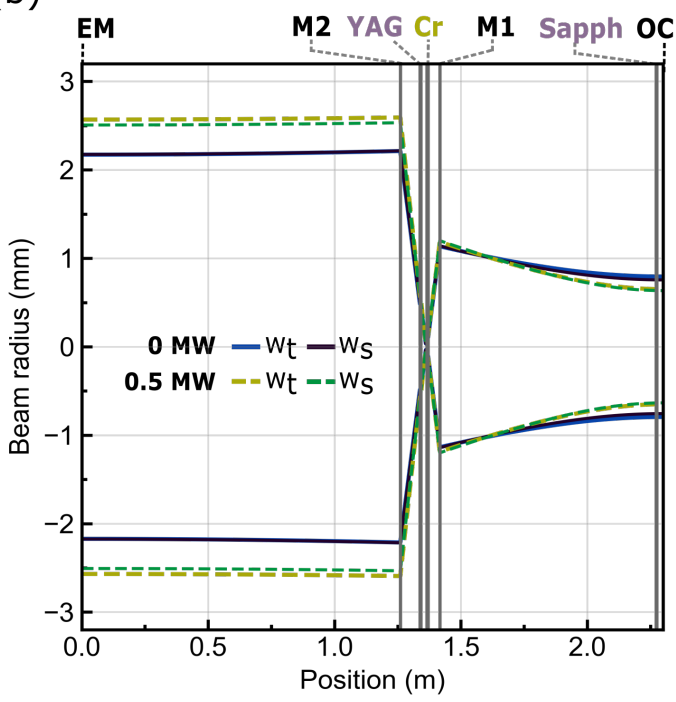

Fig. 3.12: (a) Schematic of the laser cavity and the experimental length scales. To determine the location of the oscillator's stability zones, the distance $L$ between mirror $M 2$ and the YAG plate, and also the distance between $M 2$ and $E M$ was varied, with the final position for KLM marked in orange. (b) Theoretically calculated beam radii for CW $\left(P_{0}=0 \mathrm{MW}\right)$ and mode-locked $\left(P_{0}=0.5 \mathrm{MW}\right)$ laser performance based on nonlinear ABCD-matrix analysis.

In MIRanda1, a first optimization of the intra-cavity dispersion was performed when inserting sapphire plates into the shorter resonator arm. Varying the substrate thickness from $2 \mathrm{~mm}$ to $6 \mathrm{~mm}$ in small steps $(0.5 \mathrm{~mm})$, the corresponding modelocked output spectra were analyzed with a grating-based $\mathrm{PbS}$ array spectrometer

9 This is a commonly used technique to introduce an intensity spike and initiate mode-locked operation (see simulated spiking behaviour in figure 2.2). 
(BEWW Tek, $\lambda=1500-2750 \mathrm{~nm}$ ). As illustrated in figure 3.13 (a), the slight changes in round-trip dispersion manifest themselves in varying output spectral shapes and coverage, with their full width at half maxima (FWHM) being inversely proportional to the generated pulse duration $\left(\tau_{0} \propto 1 / \Delta \lambda\right)$. Therefore, the measured FWHM ${ }^{10}$ is plotted for different sapphire thicknesses, which reveals that the broadest spectral bandwidth is generated with a $3.5 \mathrm{~mm}$ thick plate.

(a)
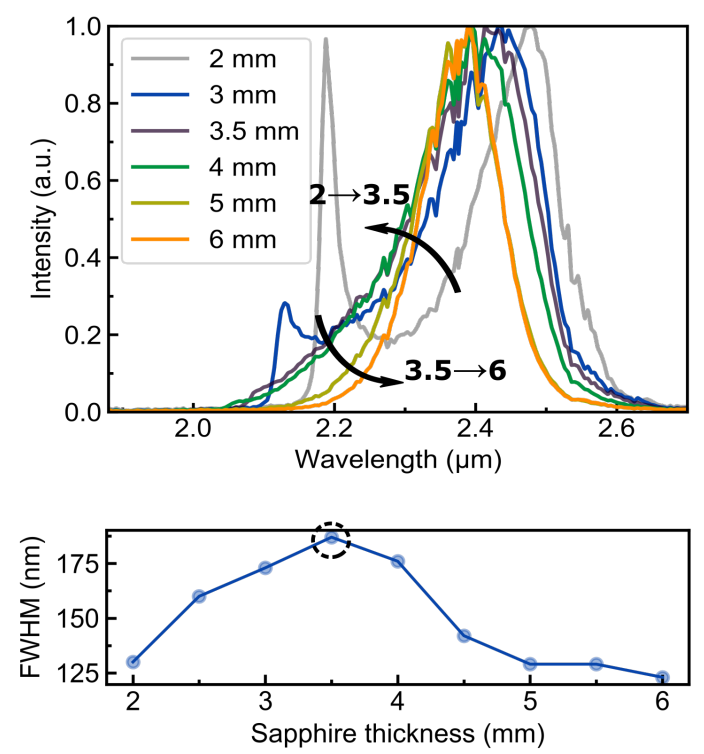

(b)
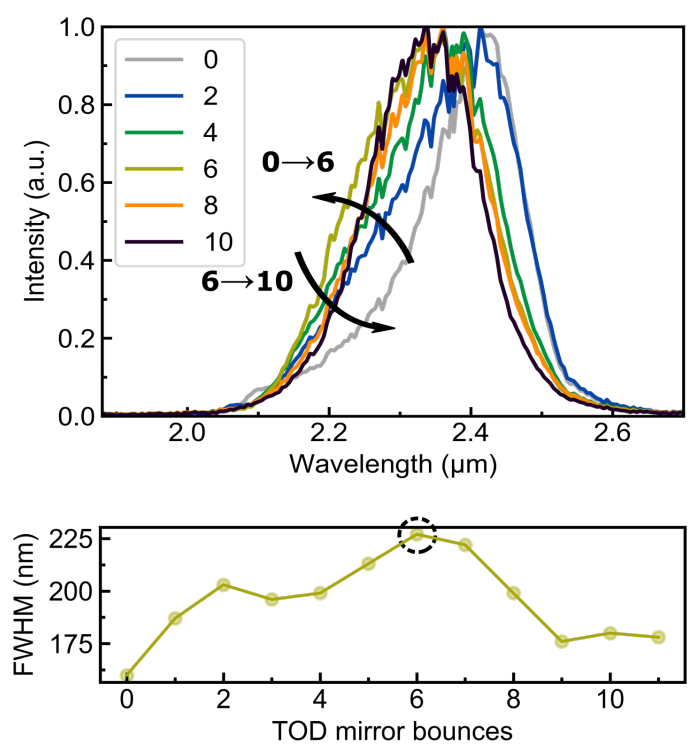

Fig. 3.13: Illustration of the intra-cavity dispersion tuning and its effect on the mode-locked spectral shape and FWHM. (a) Selected mode-locked output spectra for different thicknesses of sapphire placed into the oscillator. The pronounced sidebands for $2 \mathrm{~mm}$ and $3 \mathrm{~mm}$ are a clear indication of dispersive wave formation and a domination of strong TOD in the cavity. Also plotted are the spectral FWHM for each measurement to identify the most suitable configuration. (b) Variation of the number of TOD mirror bounces per cavity round-trip for a fixed sapphire plate thickness of $3.5 \mathrm{~mm}$. An odd number of bounces could be achieved when one of the TOD mirrors was used as an end-mirror. Similar to (a), the FWHM of the generated spectra is displayed as well.

To achieve an even broader spectral coverage, however, further dispersion tuning was needed. This became apparent when analyzing the spectral shapes for $2 \mathrm{~mm}$ and $3 \mathrm{~mm}$ thick sapphire plates more closely: here, the round-trip GDD is decreased by so much that it eventually crosses the zero dispersion line at certain wavelengths, resulting in the appearance of a resonant spectral sideband - a so-called "dispersive wave". Owing to the presence of relatively strong third-order dispersion, the soliton undergoes dispersive and periodic perturbation, and energy can be transferred from

10 The optical resolution of the fiber-coupled spectrometer was rather limited and specified as 2-10 nm. Hence, the measured FWHM spectra were only used for a qualitative comparison. 
the soliton to a narrowband resonance in the normal dispersion regime [107]. The resulting emergence of a dispersive wave has already been reported for a number of ultrafast laser systems, including KLM bulk solid-state lasers [144, 160, 226, 227] and mode-locked fiber lasers [107, 228]. In MIRanda1, the generation of dispersive waves at around 2.1-2.2 $\mathrm{mm}$ is attributed to the strong uncompensated TOD inside the cavity, which causes the round-trip dispersion to become positive at the shorter wavelengths.

To overcome this limitation, specific dispersive mirrors manufactured in-house were used. These multi-layer optics had been designed to compensate for the material dispersion of $2 \mathrm{~mm}$ sapphire and $2 \mathrm{~mm}$ ZnSe, which theoretically results in net zero dispersion at the central wavelength $\left(\mathrm{GDD}_{2.3 \mu m} \approx 0 \mathrm{fs}^{2}\right)$. Thus, they were referred to as "TOD mirrors" since they mainly addressed the higher-order dispersion of the laser resonator. To identify the amount of mirrors needed for the given cavity configuration, the mode-locked spectral FWHM was measured for a varying number of TOD mirror bounces per round-trip. Finally, even further optimization was feasible when the thickness of the sapphire plates and the number of TOD mirrors was successively adjusted until convergence was reached. Also, additional fluoride-based substrates $\left(\mathrm{CaF}_{2}, \mathrm{BaF}_{2}\right)$ were tried for finer steps in dispersion, but no further improvement was observed.

In this section, all presented spectra were taken at the same output coupling ratio of $14 \%$ with measured average output powers in the range between $200-250 \mathrm{~mW}$. Therefore, slight fine-tuning was needed when going towards ever higher output coupling rates, most likely because of the additional dispersion introduced by the different OC mirror coatings. In the end, the laser was operated at an $\mathrm{OC}$ ratio as high as $34 \%$, and delivered the shortest output pulse durations when $3 \mathrm{~mm}$ sapphire and 8 bounces on the TOD mirrors were used.

\subsubsection{Characterization of the Mode-Locked Output}

\section{Pulse duration and average power}

At a repetition rate of $64.7 \mathrm{MHz}$, the diode-pumped system delivered average output powers of over $700 \mathrm{~mW}(\mathrm{CW})$ and $500 \mathrm{~mW}$ (ML) in a fundamental single-mode operation $^{11}$. At the latter, mode-locked root mean square (RMS) power fluctuations of less than $0.12 \%$ were recorded over 3 hours when measured with a thermal

11 The maximum CW output power corresponds to laser operation at the center of stability zone I, whereas mode-locking was only obtained when moving towards the stability zone edge at decreased CW power levels of $\sim 450 \mathrm{~mW}$. 
power meter (Fig. 3.16 (a)). To specify the generated FWHM pulse duration, the dispersion of the $6.35 \mathrm{~mm}$-thick fused-silica output coupler substrate was compensated by a $5 \mathrm{~mm}$ thick ZnSe plate placed into the optical beam path. With a self-built second-harmonic frequency-resolved optical gating device (SHG-FROG, Figure 3.14) and a $10 \mu \mathrm{m}$ thick BBO crystal used for frequency doubling, the pulses were determined to be as short as 45 fs with a Fourier-transform limited (FTL) duration of $41 \mathrm{fs}$. The respective FROG error amounted to $0.7 \cdot 10^{-3}$ when retrieving on a grid size of 512.512 points. Correspondingly, this first KLM diode-pumped system is able to deliver pulse energies of up to $8 \mathrm{~nJ}$ and peak powers as high as $151 \mathrm{~kW}$ at pulse durations shorter than six optical cycles. Compared to the previous demonstration of a SESAM mode-locked diode-pumped Cr:ZnSe laser [182], it represents a ten-fold increase in average power as well as a four-fold reduction in pulse duration. Moreover, it constitutes an over 2.5-times increase in peak power over more mature fiber-laser-pumped Cr:ZnSe oscillators [163, 193].

(a)

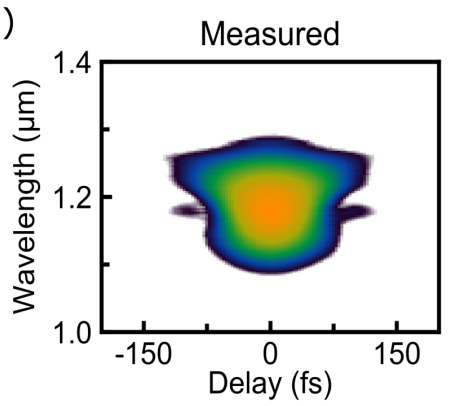

(c)

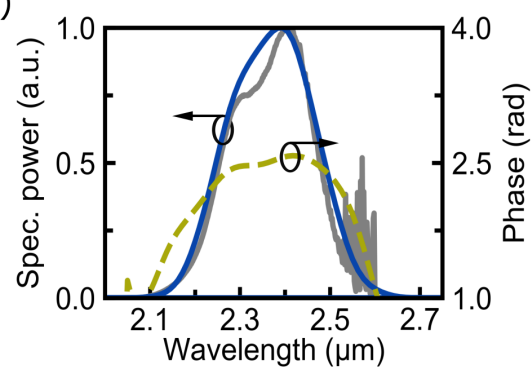

(b)

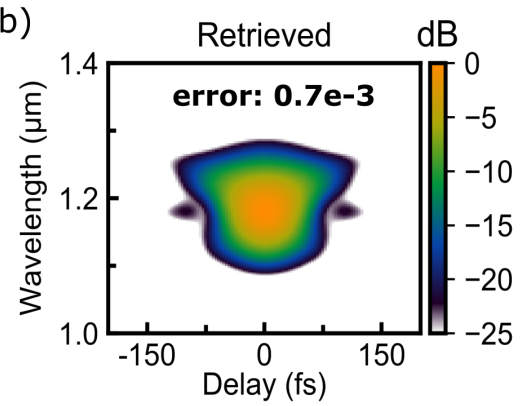

(d)

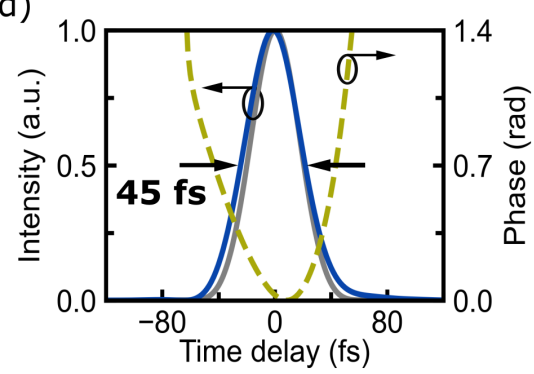

Fig. 3.14: SHG-FROG measurement of the mode-locked laser output. (a)-(b) Measured and retrieved FROG traces plotted on a normalized logarithmic intensity scale. (c) Retrieved FROG results in the spectral domain, being in good agreement with the measured output spectrum shown in grey. The latter was recorded with a high-resolution Fourier-transform-based laser spectrum analyzer (Bristol Instruments, 771B-IR) and the SHG spectra were recorded with a fiber-coupled grating-based spectrometer (Ocean Optics, NIR-512). (d) Temporal profile of the retrieved output pulse, indicating a measured FWHM pulse duration of $45 \mathrm{fs}$ (blue) and a FTL of $41 \mathrm{fs}$ (grey). 


\section{THE FIRST Directly Diode-Pumped Few-Cycle Cr-Doped II-VI Laser}

\section{Slope efficiency and output beam quality}

Similar to section 3.3.1, the mode-locked output beam quality $\left(M^{2}\right)$ was evaluated, and the horizontal and vertical $M^{2}$-factors were characterized to be nearly diffraction limited with $M^{2}<1.1$ (Figure $3.16(\mathrm{~b})$ ). By means of a wideband IR polarizer (Innovation Photonics, PGC-5), which consists of two precision polished Germanium plates at Brewster's angle and ensures a high extinction ratio of $2 \cdot 10^{5}$, the beam was measured to be polarized in the horizontal direction with an extinction ratio of at least 300. Since the oscillator's polarization cleanness is enhanced by the two intra-cavity Brewster plates, this ratio was most likely higher but hampered by the limited sensitivity at low powers for the power meter used.

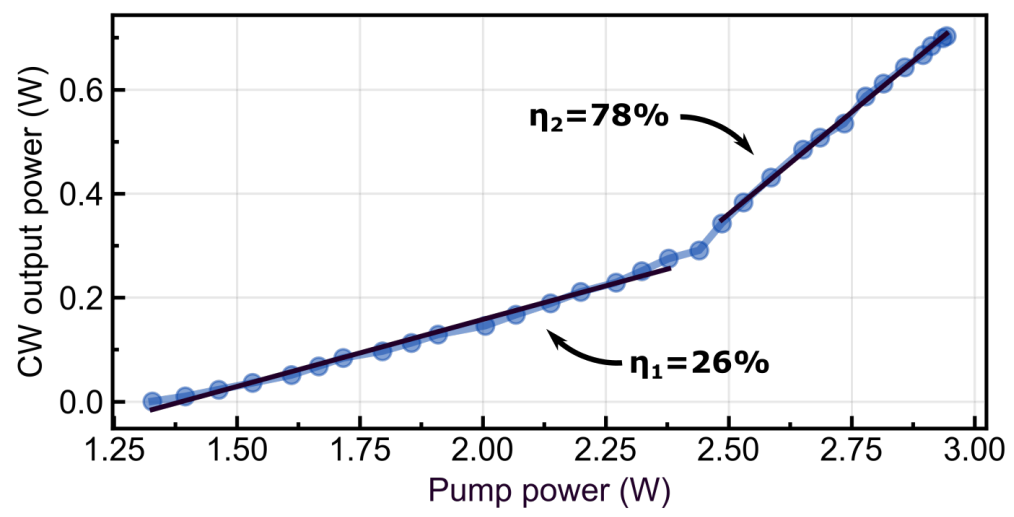

Fig. 3.15: Recorded CW slope efficiency when different pump currents were applied to increase the pump power. The break in the measured slope at around $2.5 \mathrm{~W}$ is attributed to the changing output beam characteristics of the laser diode.

When plotting the output power $P_{\text {out }}$ of a laser for different pump powers $P_{p}$, the resulting curve is typically close to a straight line above the lasing threshold $P_{t h}$. The slope efficiency $\eta$ can then be extracted as the slope of this line: $P_{\text {out }}=\eta\left(P_{p}-P_{t h}\right)$. Varying the pump power through simply adjusting the laser diode current, however, turned out to be not practical in this case. As shown in figure 3.15, the CW slope efficiency curve reveals a pronounced break at around $2.5 \mathrm{~W}$ of pump power. At this occasion, the diode output beam characteristics were examined in more detail and a changing behaviour was found for different driving currents (see section 3.3.1). Therefore, the observed break is most likely attributed to the changing mode-matching efficiency between the pump and cavity mode. To varify this assumption and to ensure a constant pump beam quality, a ND filter wheel was placed in front of the pump focusing lens. The CW slope efficiency was then measured at a fixed diode current of $10.2 \mathrm{~A}$. With the incident pump power being 
varied through filter wheel rotation, a clean slope efficiency of $\eta=50 \%$ was measured together with a slight change in the oscillator output wavelength (see figure 3.16 (c)).

(a)

(b)
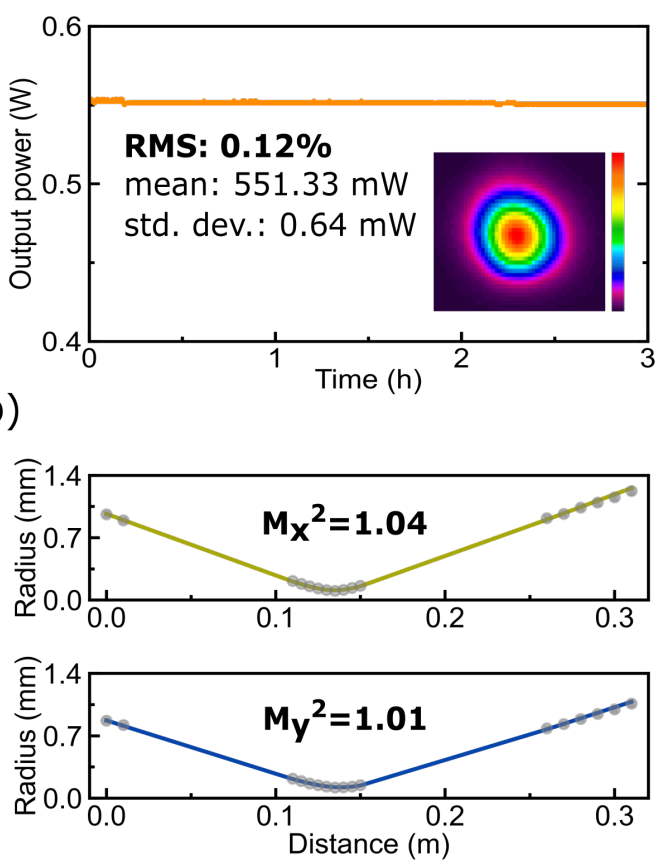

(c)
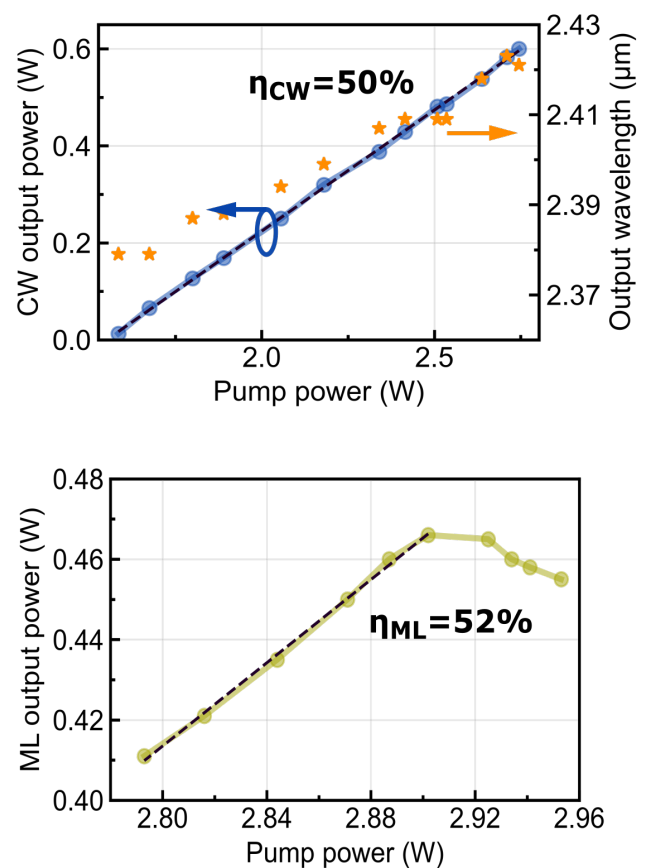

Fig. 3.16: Measured output characteristics and slope efficiencies of MIRanda1. (a) Modelocked output power stability recorded with a thermal power meter (Coherent, PS19) and corresponding beam profile measured with a pyroelectric camera (Ophir Optronics, Pyrocam III). (b) $M^{2}$-measurement performed for the horizontal and vertical axis of the mode-locked output. (c) CW and ML slope efficiency curves. For the CW slope efficiency, a ND filter wheel had to be used in the beam path to ensure constant pump beam quality (see main text).

Finally, to record a slope in mode-locked operation, the filter wheel had to be removed since it caused undesirably high losses. Only for incident pump powers $>2.8 \mathrm{~W}$, mode-locking could be initiated, and a slope efficiency of $\eta=52 \%$ was found. Beyond 2.9 watts of pump power, however, the ML output power started to drop. Besides a degradation in mode-matching efficiency, it was attributed to the excessive heating of the gain medium, which was cooled mainly from one side only. Moreover, Cr:ZnSe shares similar spectroscopic properties with Cr:ZnS but has an about 1.5-times higher thermo-optic coefficient (see table 3.1). Therefore, an improved cooling architecture as well as a change to $\mathrm{Cr}: \mathrm{ZnS}$ as gain medium was implemented in the subsequent two laser systems MIRanda2 and MIRanda3. 


\section{Amplitude noise measurements}

As highlighted in the introductory part 1.2, various applications of femtosecond MIR radiation greatly benefit from a low-noise laser performance. The output of real laser systems exhibits power (and thus intensity) fluctuations, which may be categorized as short-term or long-term [87, 229]. A degrading pump laser source, contaminated optical surfaces, or mechanical misalignment due to thermal effects are potential sources of long-term changes. In this section, however, the focus is placed on short-term fluctuations that happen within a period of less than a second.

At the most fundamental level, the discrete quantum nature of the photons results in a contribution from shot noise [230], and arises from the fact that photons are spontaneously emitted from excited atoms or ions. Consequently, these photons arrive at irregular intervals at a photodetector, and some of which are absorbed to generate photocarriers. The resulting photocurrent can then be seen as the sum of all carriers, being characterized by fluctuations in the average detected current level. Other sources of short-term fluctuations include variations in the pump laser intensity, cavity mode beating and hopping, thermal instabilities in the gain medium, mechanical vibrations of optical components, and relaxation oscillations (RO). The RO noise is characteristic for most solid-state and semiconductor lasers since small disturbances can result in large output power oscillations when the perturbing frequency components are located at the vicinity of the RO's resonance frequency $\nu_{r o}$. Therefore, excess RO noise can dominate other sources of noise, and signals of interest in spectroscopic applications get easily overwhelmed. Below $\nu_{\text {ro }}$, pump laser noise can also severely add to the noise of the oscillator. As found in section 3.2.2, rare-earth doped fiber lasers or solid-state lasers, such as those based on Cr:ZnS/ZnSe, have RO frequencies in the few $10 \mathrm{kHz}$ to a few $100 \mathrm{kHz}$ range. By contrast, semiconductor laser diodes exhibit relaxation oscillations in the GHz-domain. Using laser diodes rather than a fiber laser to pump the Cr:ZnS/ZnSe gain medium is therefore highly advantageous.

For quantifying the short-term noise of a laser system, let us consider its optical output $P(t)$ as a function of time: $P(t)=P_{a v}+\Delta P(t)$, with $P_{a v}=\langle P\rangle$ being the average output power and $\Delta P(t)$ denoting the output power fluctuations. According to the ISO Standard 11554 [231], a so-called relative intensity noise (RIN) can then be defined in the frequency domain:

$$
\operatorname{RIN}(\nu)=\frac{\left\langle\Delta P(\nu)^{2}\right\rangle}{\left\langle P(\nu)^{2}\right\rangle} \cdot \frac{1}{\Delta \nu}
$$


where the mean square power fluctuations are normalized to the mean square power as well as a noise bandwidth $\Delta \nu$. The laser RIN is fundamentally limited by the shot noise RIN, which can be formulated as [232, 233]:

$$
\mathrm{RIN}_{\mathrm{sn}}=\frac{2 h \nu_{0}}{\eta P_{a v}}=\frac{2 q}{I},
$$

where $h \nu_{0}$ is the photon energy, $P_{a v}$ denotes the average optical power, and $\eta$ is the quantum efficiency of the detector. In terms of electrical units, the generated photocurrent is $I=P_{a v} R\left(\nu_{0}\right)=P_{a v} \eta q / h \nu_{0}$ including the detector's responsitivity $R\left(\nu_{0}\right)$. Since shot noise is constant with frequency, it may be considered as a white noise source.

Typically, amplitude noise (also referred to as intensity noise) is plotted in relative terms to allow measurements taken unter different operation conditions to be compared in a straightforward manner. The noise measurements presented in this thesis are therefore plotted in logarithmic units ${ }^{12}$, and normalized to the carrier (DC level) [234, 235]:

$$
\operatorname{RIN}(\nu)[\mathrm{dBc} / \mathrm{Hz}]=\mathrm{RIN}(\nu)[\mathrm{dBm}]-10 \cdot \log (\Delta \nu)-10 \cdot \log \left(\frac{U_{D C}^{2}}{R_{\text {load }}} \frac{1}{1 \mathrm{~mW}}\right) .
$$

In the experiments, $\operatorname{RIN}(\nu)[\mathrm{dBm}]$ is the noise being measured by a radio-frequency spectrum analyzer at a certain resolution bandwidth (RBW) $\Delta \nu$. Since the power fluctuations are detected with a fast photodetector, and optical power is transferred into electrical power $\left(P_{e l} \propto P_{o p t}^{2}\right)$, the measured RMS electrical power fluctuations were normalized to the $\mathrm{DC}$ voltage $U_{D C}$. The latter is proportional to the average optical power incident on the photodetector [234], and was determined with the aid of a DC-coupled oscilloscope. Integrating the relative intensity noise over a stated frequency interval, a root mean square (RMS) value can be obtained [234, 235]

$$
\operatorname{RIN}[\% \mathrm{RMS}]=\sqrt{\int_{\nu_{1}}^{\nu_{2}} \operatorname{RIN}(\nu)[\mathrm{dBc} / \mathrm{Hz}] d \nu} .
$$

For the measurements, the amplitude noise characteristics of the mode-locked pulse trains were carefully analyzed with a photodetector attached to a high dynamicrange radio-frequency (RF) spectrum analyzer (see figure 3.17 (a)). Even though the selection of appropriate photodetectors was rather limited to record the laser output centered at 2.3-2.4 $\mathrm{mm}$, a biased InGaAs detector was found, being sensitive at the 0.9-2.6 $\mathrm{mm}$ wavelengths. Also, a high signal-to-noise ratio of $\sim 90 \mathrm{~dB}$ could be achieved for all measurements using a $50 \Omega$ termination for impedance matching. To avoid cross-coupling of beam-pointing fluctuations into the relative intensity

$\overline{12}$ Conversion formula: RIN $[\mathrm{dB}]=10 \cdot \log _{10}(\mathrm{RIN})$ 
noise (RIN) measurements [236], the beam was focused onto the detector area $(\varnothing 1 \mathrm{~mm})$. Mixing artefacts generated by the RF spectrum analyzer were reduced by filtering the electronic DC signal at the analyzer input. Also, a neutral density (ND) filter wheel was placed into the beam path to keep the optical signal just below the detector's saturation level ${ }^{13}$. By setting the measurement step size to $0.5 \cdot \mathrm{RBW}$, a good overlap of the data points was ensured, and a better estimate of the mean-squared quantities was obtained when averging over 20 traces taken within a certain RBW.

(a)

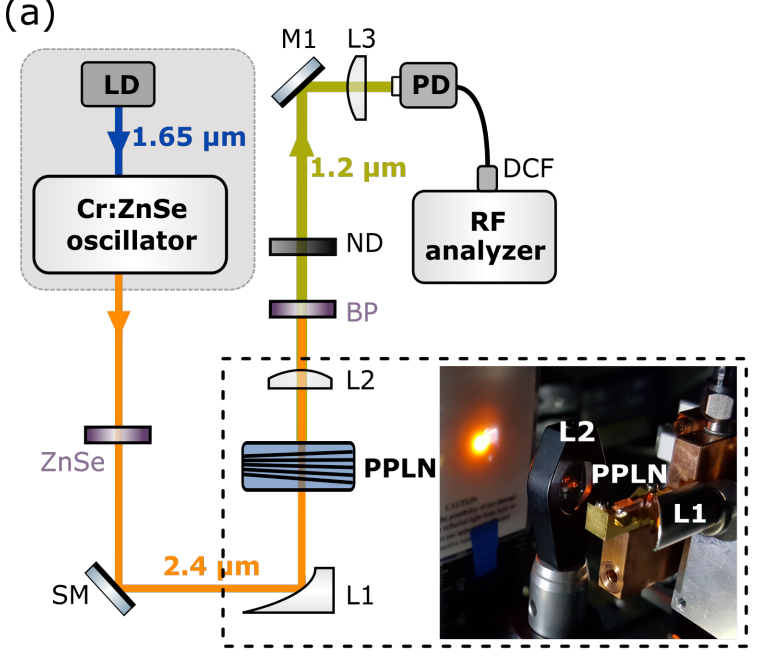

(b)

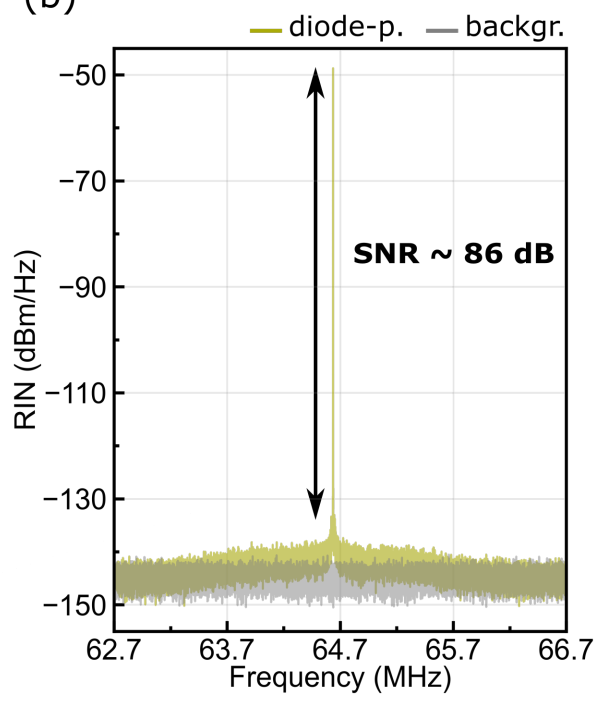

Fig. 3.17: (a) Setup for measuring the mode-locked amplitude noise of the frequency-doubled oscillator output. LD: laser diode, ZnSe: $5 \mathrm{~mm}$ AR-coated substrated for temporal pulse recompression, SM: silver mirror, L1: parabolic silver mirror $(\mathrm{f}=7 \mathrm{~mm})$, PPLN: periodically-poled lithium niobate crystal for second-harmonic generation (HC Photonics, SC1705100107, fan-out grating $\Lambda=21-35 \mu \mathrm{m}), \mathrm{L} 2$ : N-BK7 lens $(\mathrm{f}=15 \mathrm{~mm})$, BP: band-pass filter at $1.2 \mu \mathrm{m}$ (Thorlabs, FB1200-10), ND: neutral density filter wheel, M1: dielectric mirror (Thorlabs, BB1-E03), L3: AR-coated UVFS lens ( $\mathrm{f}=50 \mathrm{~mm}$ ), PD: biased InGaAs photodetector (Thorlabs, DET10D2), DCF: DC block filter (Thorlabs, EF500), RF analyzer: radio-frequency spectrum analyzer (Agilent, E4447A). The SHG generation in the PPLN crystal was captured with a camera. (b) RF spectrum of the carrier region, where the grey curve indicates the background noise floor of the measurement. High signal-to-noise ratios close to $90 \mathrm{~dB}$ were obtained for the frequency-doubled output, comparable to the measurements at the fundamental wavelengths.

The laser amplitude noise was then analyzed in the low-frequency range between $20 \mathrm{~Hz}$ to $1 \mathrm{MHz}$, also referred to as the baseband [235]. Here, an average optical power of less than $1 \mathrm{~mW}$ and a corresponding DC voltage of $47 \mathrm{mV}$ were sufficient

13 When the photodetector signal was monitored with a DC-coupled oscilloscope (Teledyne LeCroy, HDO4034), a saturation of the detector manifested itself in a deformation of the resolved pulse train shape. 
to obtain high signal strengths without saturation. As defined in formula 3.6, the amplitude noise of the direct oscillator output was measured first and plotted in relative units of $\mathrm{dBc} / \mathrm{Hz}$. Similarly, the background noise of the measurement devices was obtained when blocking the optical signal into the detector. Even though the photodetector was already operating at the limit of the linear regime, the laser amplitude noise was surprisingly close to the background noise floor. Therefore, a periodically poled lithium niobate (PPLN) crystal was used to take additional data on the frequency-doubled output after second-harmonic generation (see figure 3.17). The quadratic dependence of the SHG frequency conversion process on the optical input power should enhance the RIN by a factor of two [234, 237], corresponding to an increase of $6 \mathrm{~dB}$ in electrical power. Moreover, to only measure the noise centered at $1.2 \mu \mathrm{m}$ and block contributions from the fundamental and other wavelengths, a suitable bandpass filter was placed right behind the crystal. Similar to the measurements at the fundamental wavelength, it resulted in a DC-voltage of about $47 \mathrm{mV}$, which corresponds to an optical power of $P_{a v}=U_{D C} /\left(R(\lambda) R_{\text {load }}\right)=47 \mathrm{mV} /(0.39 \mathrm{~A} / \mathrm{W} \cdot 50 \Omega) \approx 2.4 \mathrm{~mW}$ at $1.2 \mu \mathrm{m}$. In figure 3.17 (b), the RF spectrum of the carrier region is depicted, revealing a correspondingly high signal-to-noise ratio of close to $90 \mathrm{~dB}$ similar to the measurements at the fundamental output.

The measurement results are shown in figure 3.18 (green curves) and were obtained for two different resolution bandwidths and frequency spans. At the very low frequencies up to about $200 \mathrm{~Hz}$, the noise spectrum exhibits a $f^{-1}$-behaviour, as it has been observed as resistance fluctuations of metals or semiconductors [238-241]. Beyond $1 \mathrm{kHz}$, a more "plateau"-like noise structure without clear noise peak is seen instead, which falls to the background noise near $2 \mathrm{MHz}$. This can be attributed to the short fluorescence lifetime of $\mathrm{Cr}: \mathrm{ZnSe}$, which is on the order of a few microseconds (see table 3.1). As calculated in section 3.2.2, the relaxation oscillation frequency of $\mathrm{Cr}: \mathrm{ZnS} / \mathrm{ZnSe}$ is located at hundreds of $\mathrm{kHz}$, and any noise at higher frequencies will be damped because of the filtering effect of the gain medium. Pump laser noise at lower frequencies, however, can be transferred to oscillator noise, and it is worthwile to note that the usual noise peak originating from relaxation oscillations in the rare-earth doped (erbium/thulium) pump fiber lasers at hundreds of $\mathrm{kHz}$ [193] is absent in this directly diode-pumped system. In fact, similar plateau-like structure has also been observed for diode-pumped Ti:sapphire oscillators, and was attributed to the relaxation oscillations of the Ti:sapphire laser itself [234, 235].

To further investigate the transfer of pump noise, the pump source of MIRanda1 was changed to a commercial Erbium fiber laser (Bktel, HPFL-370-1567-FCAPC) that has been connected to the same power supply as the InP pump diode. The direct noise output of this fiber laser has already been shown in figure 3.4 when comparing the amplitude noise of different available pump laser sources. It should 
be noted, however, that this laser is not meant to represent state-of-the-art lownoise fiber lasers, and it was also not optimized for low-optical-noise performance. Nevertheless, it serves to illustrate the effect of a noisy pump source on the output noise characteristics of a $\mathrm{Cr}: \mathrm{ZnS} / \mathrm{ZnSe}$ laser. The respective measurements (blue curves, Fig. 3.18) were now taken at the fundamental wavelength without further noise-enhancing frequency doubling. The resulting amplitude noise of the fiberlaser-pumped Cr:ZnSe laser is now notably higher, with spectral features such as the noise peak at $40 \mathrm{kHz}$ clearly originating from fiber laser noise being transferred to oscillator noise. A similar observation is also presented in reference [193].
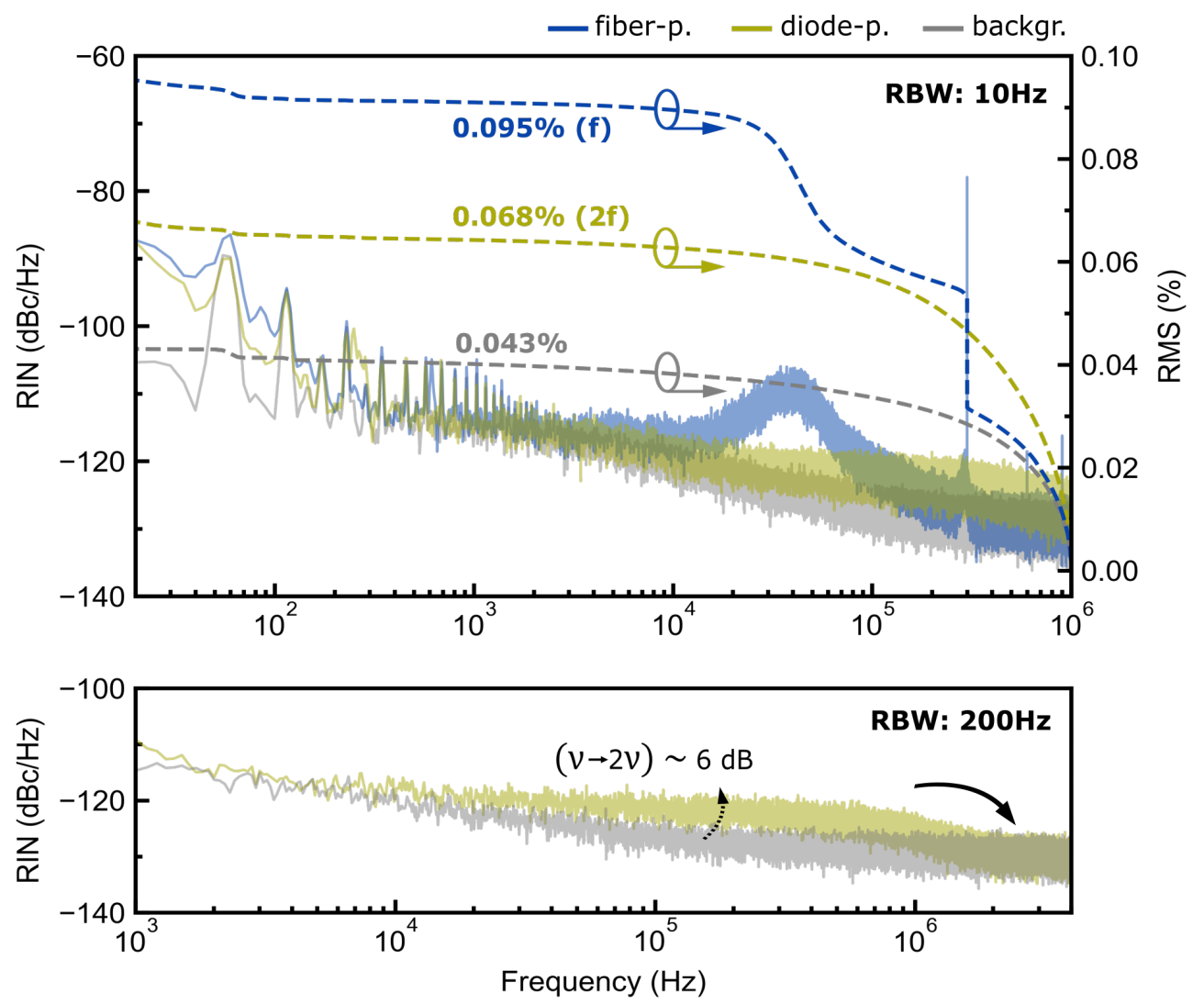

Fig. 3.18: Amplitude noise measurements and integrated RMS noise of the frequencydoubled (2f) output of MIRanda1 (green, diode-pumped). For comparison, the data of the fundamental (f) is added when the oscillator was pumped by a commercial fiber laser instead (blue). Both the fiber laser and laser diode were driven by the same low-noise current source (Delta Elektronika, SM 70-AR-24). The RIN was also measured in a broader span up to $4 \mathrm{MHz}$, and illustrates the drop in noise signal to the background noise floor at frequencies around $2 \mathrm{MHz}$ - attributed to the filtering effect of the gain medium.

Finally, for a quantitative comparison between the diode- and fiber-pumped $\mathrm{Cr}: Z n S e$ laser, the integrated RMS noise was calculated for the measured frequency 
span from $20 \mathrm{~Hz}$ to $1 \mathrm{MHz}$ according to formula 3.7. The lower integration limit was set to $20 \mathrm{~Hz}$ since the RF spectrum analyzer itself exhibited undesirably high noise below $20 \mathrm{~Hz}$, even when the signal input was simply terminated with a $50 \Omega$ resistor. The integrated RMS noise level was then calculated to be $0.095 \%$ for the fiber-pumped system, with $0.043 \%$ being attributed to the background noise floor. By contrast, the integrated RIN of the directly diode-pumped laser system was measured to be $0.068 \%$ after frequency doubling, and should be even lower for the fundamental wavelengths. This clearly illustrates the highly promising low-noise performance and potential of directly diode-pumped Cr:ZnS/ZnSe lasers. Since the calculated shot noise level was at $-154 \mathrm{dBc} / \mathrm{Hz}$ and the background noise floor was measured to be $-130 \mathrm{dBc} / \mathrm{Hz}$, further optimization in the noise measurement setup was required for future noise studies. In the noise measurements for MIRanda2 and MIRanda3, an improved measurement setup will be presented, eventually revealing the mode-locked noise of the diode-pumped system directly at the $2-3 \mu \mathrm{m}$ wavelengths.

\subsubsection{The Success of Powerful Diode-Pumped Operation}

At first sight, it sounds remarkable that high continuous-wave average powers and soft-aperture KLM - both achieved in a clean spatial TEM $_{00}$ output mode - can be supported by a solid-state laser cavity which is pumped by the non-diffractionlimited output of a single wide stripe laser diode. According to the conventional understanding [102], the self-focusing inside the gain crystal allows the cavity mode to be smaller in size when the oscillator is generating ultrashort pulses. This results in an improved overlap between the pump and cavity mode, typically yielding higher output powers in mode-locked operation. In the case of MIRanda $1^{14}$, however, the pump beam diameter inside the Cr:ZnSe crystal roughly equals the laser mode in the fast axis direction, but is much larger in the slow axis direction. Therefore, it cannot per se provide a higher gain for a shrinking cavity mode. Experimentally, soft-aperture mode-locking was successfully implemented together with high average powers in CW and ML laser operation, and some potential reasons are listed below to help explain the observed efficiency and stability (see also references $[206,207]$ ).

\section{Mode diameters in the crystal:}

In section 3.3.1, an ABCD-matrix simulation of the experimental setup was presented for continuous-wave (0 MW) and pulsed (0.5 MW) operation, and an enlarged section of the focal region is now shown in figure 3.19 (a). Interestingly, the pulsed laser mode diameter inside the gain crystal is about $30-32 \mu \mathrm{m}(15-18 \%)$ larger than

14 This is also true for the other two laser systems that will be presented in this thesis. 
the CW mode in the sagittal and tangential direction for the given set of parameters. By a proper adjustment of the pump focus position, the pump mode is therefore able to act as a soft aperture: because the cavity mode in the Cr:ZnSe crystal increases upon mode-locked operation, it allows for a slightly improved overlap with the pump beam and thus increased laser gain. This explanation is supported by the experimental observation that the oscillator output power increased by a factor of $10-15 \%$ upon mode-locking.

(a)

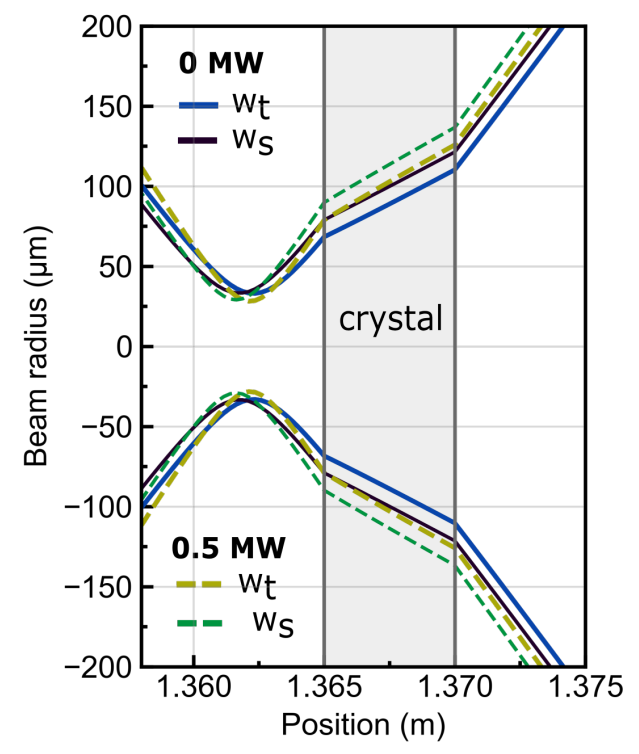

(b)
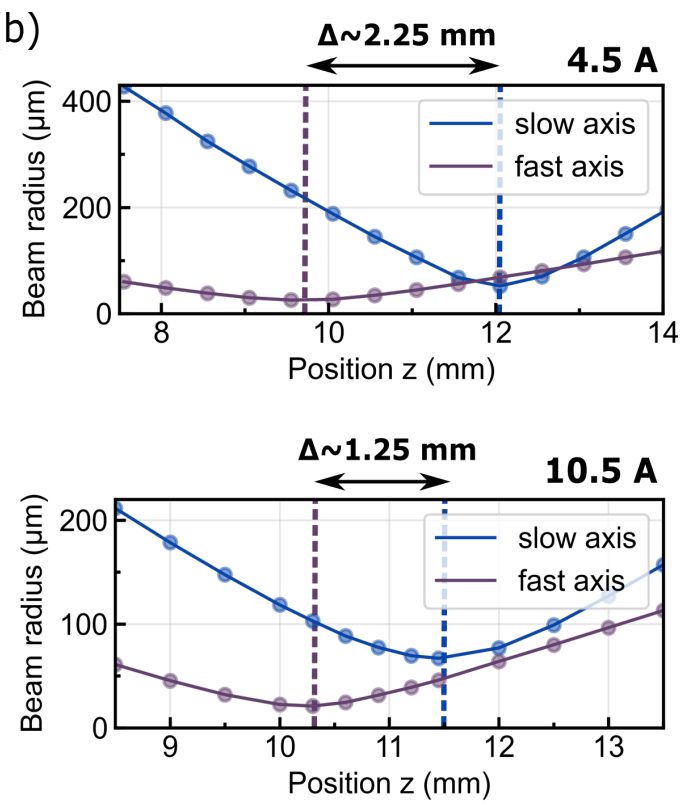

(c)

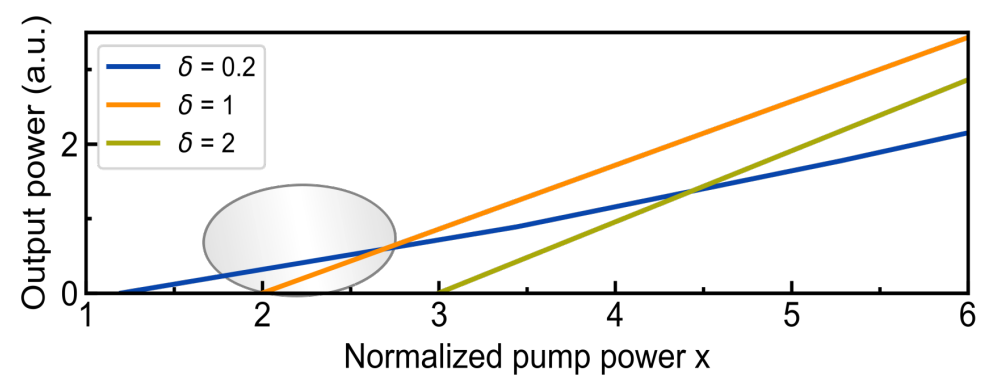

Fig. 3.19: (a) Simulated tangential and sagittal beam radii of the CW and ML cavity mode in the focusing region around the crystal position. The calculations are based on (non)linear ABCD-matrix simulations for two different intra-cavity peak powers. (b) Measured pump beam radii around the focus when the diode is pumped at low current $(4.5 \mathrm{~A})$ as well as at the current that was finally used for operation of MIRanda1 (10.5 A). (c) Theoretical laser output power versus normalized pump power for different relative cavity and pump mode sizes $\left(\delta=\left(w_{0} / w_{p}\right)^{2}\right)$. 


\section{Pump focusing characteristics:}

Another effect is depicted in figure 3.19 (b): after the cavity and pump setup parameters had been optimized for highest CW output power and best KLM performance - with the corresponding output results shown in the previous section - the actual focusing characteristics of the pump beam were analyzed in more detail. For practical reasons, the pump beam was deflected and sent into a separate setup, mimicing the beam path up to the focusing region. With the scanning slit profiler in place, the $D_{4 \sigma}$ beam diameters were measured around the focus for both beam directions. Even though a slight difference was observed when operating the diode at two different pump currents, which is attributed to the changing $M^{2}$-value and beam divergence, it reveals a clear discrepancy of $\Delta>1 \mathrm{~mm}$ in the actual focus position of the fast and slow axis. Indeed, this might be more favorable with respect to mode-matching and mode-locking since it helps to increase the effective Rayleigh length of the pump beam. Consequently, the resulting improvement in the overlap volume betweeen the fundamental cavity beam and the pump beam allows for higher average output powers ${ }^{15}$.

\section{Output power versus cavity/pump mode ratio:}

Under certain conditions, it might actually be advantageous to have a pumped area that is larger than the one spanned by the fundamental laser mode in the gain medium. In fact, chapter 7 of reference [86] presents a relationship between the continuous-wave output power $P_{\text {out }}$ and the pump power $P_{p}$ for a given Gaussian pump beam radius $w_{p}$ and cavity mode size $w_{0}$. For the sake of simplicity, it is assumed that both beam radii remain nearly constant over the crystal length. Also, when assuming a Gaussian pump distribution, a spatial dependence of the gain on radial and longitudinal coordinates is included in the mathematical calculations. For the analysis, a normalized output power $y=P_{\text {out }} / P_{S}$ and a normalized pump power $x=P_{p} / P_{m t h}$ are defined, where $P_{S}$ and $P_{m t h}$ are the saturation power and the minimum threshold power according to:

$$
P_{S}=\frac{\gamma_{2} \pi w_{0}^{2}}{4} I_{S}, \quad P_{m t h}=\left(\frac{\gamma}{\eta_{p}}\right)\left(\frac{h \nu_{p}}{\tau}\right)\left(\frac{\pi w_{p}^{2}}{2 \sigma_{e m}}\right) \quad \text { for } w_{0} \ll w_{p}
$$

These quantities depend on the single pass loss $\gamma$ of the cavity, which includes the logarithmic loss $\gamma_{2}$ due to the transmission of the output coupling mirror, as

15 Note that the position of the cylindrical pump lenses was optimized to reach the highest CW output power in single-mode laser operation, and was also found to be ideal for mode-locked operation. 
well as the pump efficiency $\eta_{p}$, the saturation intensity $I_{S}$, the upper-state lifetime $\tau$, and the emission cross-section $\sigma_{e m}$. After some mathematical calculations (see Appendix E in reference [86]), the resulting relation between $x$ and $y$ can be written as:

$$
\frac{1}{x}=\int_{0}^{1} \frac{t^{\delta}}{1+y t} d t
$$

with $\delta=\left(w_{0} / w_{p}\right)^{2}$. This equation was solved analytically for three different cases, and the results are plotted in figure 3.19 (c): (i) an equal pump and laser mode size $(\delta=1)$, (ii) a slightly larger laser mode than pump mode $(\delta=2)$, and (iii) a pump mode being almost twice as large as the cavity mode $(\delta=0.2)$. Even though lasers are typically operated at values $x \gg 1$, this does not necessarily hold for the rather low pump powers considered in this thesis, and $x \approx 2$ is a good approximation then. As indicated by the grey circle in figure 3.19 (c), the lasing threshold could not only be reduced for $\delta<1$, but also the output power is possibly larger when operating near the minimum threshold (around $x \approx 2$ ) - even though the CW and ML cavity mode are smaller than the pump mode.

\subsection{Chapter Summary}

With the non-diffraction limited output of a single-emitter laser diode, a directly diode-pumped few-cycle $\mathrm{Cr}^{2+}$-doped II-VI oscillator was demonstrated for the first time. Delivering a mode-locked output power of $500 \mathrm{~mW}$ and pulse durations of $45 \mathrm{fs}$, it constitutes a sixty-fold increase in peak power over the previous record based on SESAM mode-locking [182] (see table 3.4), and a more than 2.5-times improvement when compared to more mature fiber-laser-pumped versions [163, 193] Moreover, noise measurements confirmed an excellent amplitude noise performance, with a measured integrated RIN of less than $0.068 \%$ RMS $(20 \mathrm{~Hz}-1 \mathrm{MHz})$ for the frequency-doubled output.

In section 3.1, Cr-doped II-VI crystals were presented to be highly attractive candidates for the direct generation of ultrashort femtosecond pulses in the 2-3 $\mathrm{mm}$ spectral range. With favorable material and spectroscopic characterstics similar to Ti:sapphire — one of the most popular near-infrared laser materials - they are commonly referred to as the "Ti:sapphire of the mid-infrared" [151]. As presented in section 3.3, pulse durations shorter than six optical cycles at $2.4 \mu \mathrm{m}$ were directly generated via soft-aperture Kerr-lens mode-locking. The latter, however, relies on the action of a Kerr lens inside the gain crystal, which renders the approach of direct diode-pumping highly challenging for ultrafast and stable mode-locking. When careful considerations regarding laser diode selection, mode-matching as 
well as the overall mechanical design (section 3.2) are made, the highly divergent and elliptic output beam of a low-brightness single-emitter laser diode can be successfully implemented in an ultrafast KLM-based Cr:ZnS/ZnSe laser. MIRanda1 thus represents a new class of few-cycle, low-noise, and table-top laser systems, which can serve as a highly attractive and accessible route to generate coherent radiation in the $2-3 \mu \mathrm{m}$ range, and also in the deeper MIR (see chapter 5).

\begin{tabular}{l|cc}
\hline & Previous record [182] & This work [209] \\
\hline ML mechanism & SESAM ML & Kerr-lens ML \\
Gain medium & Cr:ZnSe $(4 \mathrm{~mm})$ & Cr:ZnSe $(5 \mathrm{~mm})$ \\
$P_{\text {pump }}$ & $3.5 \mathrm{~W}$ & $3 \mathrm{~W}$ \\
$\lambda_{\text {pump }}$ & $1.55 \mu \mathrm{m}$ & $1.65 \mathrm{\mu m}$ \\
$P_{C W}$ & $340 \mathrm{~mW}$ & $700 \mathrm{~mW}$ \\
$P_{M L}$ & $50 \mathrm{~mW}$ & $500 \mathrm{~mW}$ \\
$\tau_{0}$ & $180 \mathrm{fs}$ & $45 \mathrm{fs}$ \\
$\nu_{\text {rep }}$ & $100 \mathrm{MHz}$ & $64.7 \mathrm{MHz}$ \\
OC ratio & $5 \%$ & $34 \%$ \\
\hline$E_{0}$ & $\mathbf{0 . 5 0} \mathbf{~ n J}$ & $\mathbf{7 . 7} \mathbf{~ n J}$ \\
$P_{0}$ & $\mathbf{2 . 4 4} \mathbf{~ k W}$ & $\mathbf{1 5 1} \mathbf{~ k W}$ \\
\hline
\end{tabular}

Table 3.4: Comparison of the output characteristics of the previous record versus the first demonstration of diode-pumped few-cycle $\mathrm{Cr}^{2+}$-doped II-VI oscillators. $\tau_{0}$ : measured output pulse duration, $E_{0}$ : pulse energy, $P_{0}$ : peak power. For calculating the peak power of solitonic pulses ( $\mathrm{sech}^{2}$-shape), the following formula was used: $P_{0}=0.88 \cdot P_{M L} /\left(\tau_{0} \cdot \nu_{r e p}\right)$ [242]. 



\section{REACHING THE PERFORMANCE OF StATE-OF-THE-ART Fiber-Pumped Systems}

The advancement of femtosecond solid-state laser technology along with its downstream applications is greatly affected by the average and peak power scalability of existing laser systems. With powerful few-cycle oscillators, not only the effectiveness of laser-driven nonlinear optical processes can be dramatically enhanced but also their respective threshold gets notably reduced - being of fundamental interest for the envisaged mid-IR generation. In table 2.1 of the theoretical section 2.1.2, an overview of relevant nonlinear processes based on $\chi^{(2)}$ - and $\chi^{(3)}$-related effects was provided, which are triggered by a material's nonlinear response under intense optical excitation.

Considering state-of-the-art fiber-laser-pumped Cr:ZnS/ZnSe laser systems, their average output and peak power levels are already sufficient to drive a series of nonlinear $\chi^{(2)}$ optical processes, including recently demonstrated intra-pulse DFG [33, 167]. First reported in reference [243], this robust single-beam technique provides one of the most promising routes for generating broadband coherent mid-infrared radiation, which is passively CEP-stabilized. Moreover, a powerful few-cycle Cr:ZnS/ZnSe driving laser not only improves the MIR bandwidth and power levels that can be obtained upon IDFG, but also improves the effectiveness of nonlinear spectral broadening in fibers [73]. For example, the extent of frequency shifts for Ramaninduced soliton self-frequency shifting is inversely proportional to the 4th power of the driving pulse duration, while a simultaneous reduction in soliton order (see figure 4.1) results in compressed MIR pulses with less pedestal as well as supercontinua featuring higher spectral coherence [244]. With demonstrated conversion efficiencies around 50\% [73], fiber spectral broadening is particularly interesting for covering the shorter-wave mid-infrared around (3-5 $\mathrm{mm})$, being host to the molecular fingerprints of many hydrocarbons, or greenhouse and atmospheric gases [245].

Combining the salient advantages of powerful and ultrafast driving laser systems with the benefits of directly diode-pumped Kerr-lens mode-locked $\mathrm{Cr}^{2+}$-doped II-VI oscillators has motivated the set up of a second diode-pumped system - MIRanda2, which will be presented in this chapter. Driven by the results achieved in chapter 3 as well as by the goal to reach and surpass the performance of state-of-the-art and more mature fiber-laser-pumped systems, the following sections serve to illustrate the 
power-scalability of direct diode-pumping. Also, major challenges will be presented along with design and performance improvements achieved over the first generation.

(a)

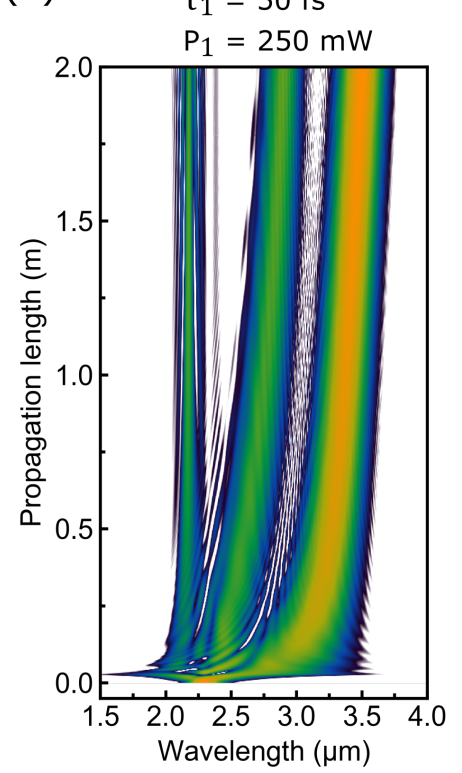

(b)

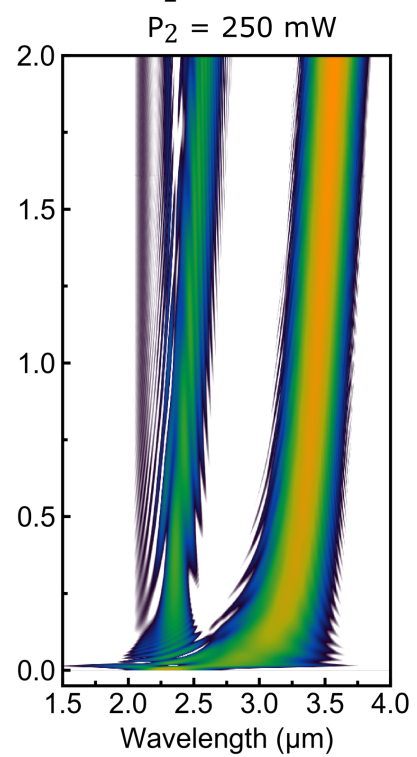

(c)

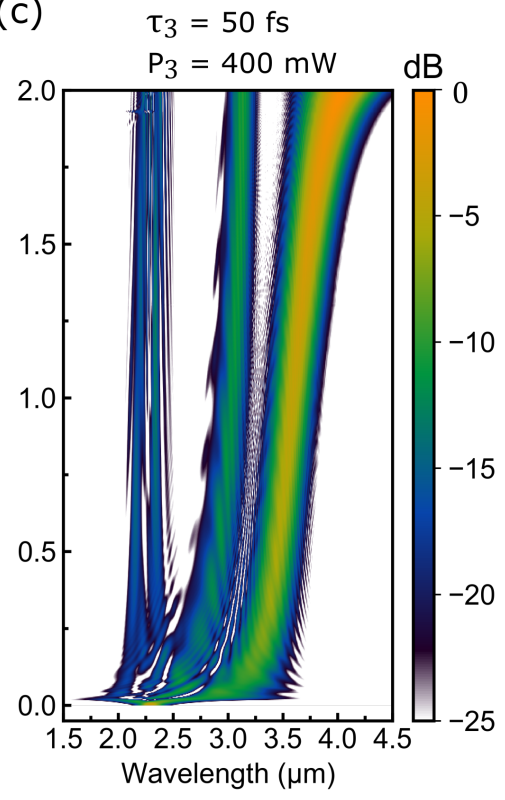

Fig. 4.1: Simulated pulse propagation in a ZBLAN fiber (6.8 $\mu \mathrm{m}$ core diameter, 2 meters), illustrating the effect of input power and pulse duration on Raman-induced soliton self-frequency shifting. The simulations were performed with the software fiberdesk, and the parameters were taken from [73]. In (a), a solitonic shift from 2.3 to $3.5 \mu \mathrm{m}$ is shown, along with a second but substantially weaker soliton as well as some dispersive wave generation around $2.1 \mu \mathrm{m}$. In (b), an input pulse with half the pulse duration is used, resulting in a slightly more red-shifted $(3.6 \mu \mathrm{m})$ soliton. Most importantly, less energy is shed into dispersive waves or secondary solitons, given the reduction in soliton order. In (c), a simulation was performed for a higher input power level, resulting in an even stronger shift up to $4.0 \mu \mathrm{m}$. This clearly illustrates the advantage of using higher average powers combined with shorter pulse durations for nonlinear optical processes such as the nonlinear spectral broadening in fibers.

\subsection{Challenges of Reaching Higher Average and Peak Powers}

The emission of a single-emitter laser diode was sufficient to demonstrate the successful combination of soft-aperture Kerr-lens mode-locking with diode-pumping, resulting in $500 \mathrm{~mW}$ of stable and low-noise output pulse trains. To approach the watt-level, however, several additional challenges arise and need to be addressed. In this section, a focus will be placed on the increase in continuous-wave average power via pump beam polarization-multiplexing, along with the effects of higher intra-cavity peak powers on the KLM performance. 


\subsubsection{Pump Setup Considerations}

Scaling the continuous-wave output power of fiber-laser-pumped Cr:ZnS/ZnSe systems is typically accomplished through an increase in the pump laser's output power. Thanks to their applicability to telecommunications and their widespread commercial availability, Erbium-doped fiber lasers $(\lambda=1.5 \mu \mathrm{m})$ have become one of the most attractive pump sources for Cr:ZnS/ZnSe lasers. With up to $10 \mathrm{~W}$ of available pump power, watt-level $\mathrm{CW}$ and mode-locked systems have already been demonstrated [64, 246]. By contrast, the power scalability of directly diode-pumped $\mathrm{Cr}: \mathrm{ZnS} / \mathrm{ZnSe}$ oscillators is strongly hampered by the relatively low output power of single-emitter laser diodes. Taking into account the decreased beam quality at higher driving currents (see section 3.3), the effective output power of one single InP diode $(\lambda \approx 1.55-1.65 \mu \mathrm{m})$ is limited to 2.5 watts to 3 watts. Therefore, the only reasonable way to increase the overall pump power is the incorporation of two single-emitter laser diodes into the pump setup.

Compared to Ti:sapphire crystals, which are known to exhibit a polarizationdependent absorption cross-section [158, 247], the absorption of Cr:ZnS/ZnSe gain media can be regarded as optically isotropic. Since no significant change is observed for varying pump polarization [214], the linearly polarized emission of two single-emitter laser diodes can be combined into a single beam geometry to pump the $\mathrm{Cr}: \mathrm{ZnS} / \mathrm{ZnSe}$ crystal. Moreover, polarization-multiplexing is a great advantage over diode-pumped Ti:sapphire systems, which typically rely on spatial multi-plexing [207] or counter-propagating pump schemes [207, 208, 248], with the latter potentially causing detrimental feedback from one diode into the other.

(a)

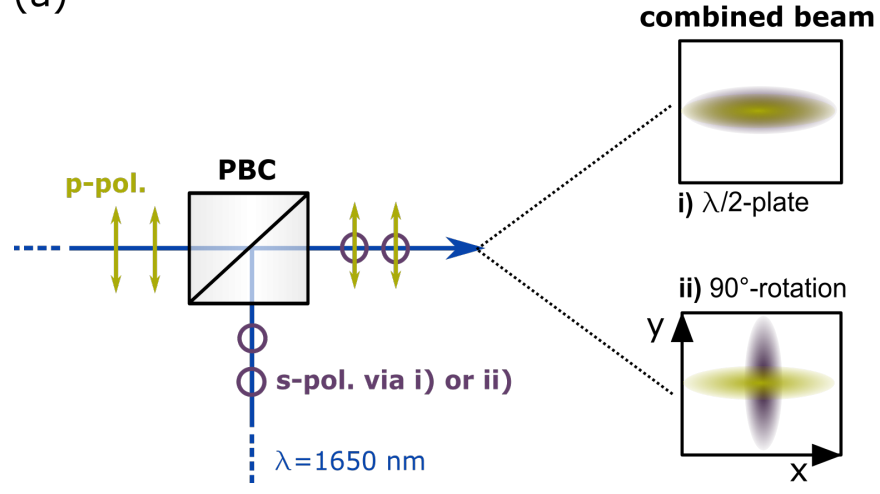

(b)

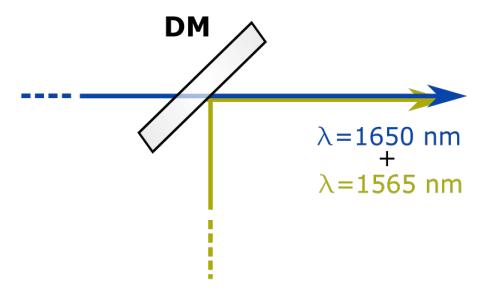

Fig. 4.2: Two ways to increase the effective pump power in a Cr:ZnS/ZnSe system pumped by the combined emission of single-emitter laser diodes: (a) polarization-multiplexing with the aid of a polarization beam combiner $(\mathrm{PBC})$ or (b) wavelength-multiplexing with a dichroic mirror (DM). To rotate the polarization of one diode with respect to the other, either a $\lambda / 2$-wave plate can be used or one diode mount is turned by $90^{\circ}$. These two methods, however, result in completely different combined pump beam shapes, as illustrated in (a). 
To experimentally realize a polarization-multiplexed setup, the horizontally and vertically $(\mathrm{p}-/ \mathrm{s}-)$ polarized beams of two single-emitter diodes can be united via a polarizing beam combiner (PBC). Because single-emitter diodes feature intrinsic polarization along the slow axis (horizontal) direction, the polarization of the second diode needs to be rotated via a $\lambda / 2$-wave plate. In principle, the same effect could also be achieved when rotating the diode base by $90^{\circ}$ [249]. But, as indicated in figure 4.2 (a), the combined emission of two elliptical pump beams would then form a cross rather than a line. Even though this configuration was not experimentally tested, it might be less ideal for soft-aperture Kerr-lens mode locking, which requires a tight focus in at least one direction for a better mode-matching in pulsed operation [206]. Beside the effect of polarization, also wavelength-multiplexing [248] of commercially available InP diodes lasing at $\lambda=1650 \mathrm{~nm}$ and $\lambda=1565 \mathrm{~nm}$ would be possible (see figure 4.2 (b)). However, this approach was not considered for the second-generation system, since the absorption cross-section and thus the total absorbed pump power inside the gain medium is higher when two laser diodes at $1.65 \mathrm{\mu m}$ are combined. Nevertheless, it might be of particular interest for future diode-pumped laser amplifiers, in which simultaneous polarization- and wavelengthmultiplexing can boost the average power levels of diode-pumped Cr:ZnS/ZnSe systems to ever higher levels, while retaining the overall low-noise performance.

Once set up, the beam divergences of both diodes and both axis directions need to be matched to focus to the same plane inside the gain crystal. This can be achieved by slight variations of the cylindrical and aspheric lens positions of the two laser diodes. Also, one pump beam path needs to be equipped with two additional steering mirrors to overlap the focal spot positions. Otherwise, the effective pump area is increased and the excitation of unwanted higher-order modes inside the cavity is highly likely.

\subsubsection{Effects on the Mode-Locking Performance}

From a very basic point of view and without thermal issues, one might expect that the intra-cavity pulse energy and peak power in a single-pulse and fundamental $\mathrm{TEM}_{00}$ laser operation depend linearly on the incident pump power. While this is certainly true for moderate power levels or a large net-negative intra-cavity dispersion with $\tau_{0} \propto \sqrt{\left|D_{2}\right|}$ (see section 2.2.3), excessive Kerr nonlinearities and decreased KLM action at higher power and shorter pulse durations can drive soliton mode-locking unstable. Once this limit is reached, any further increase in peak power might either result in a breakthrough of a continuous-wave component and its further enhancement or the appearance of multiple pulses - setting a limit to the shortest achievable pulse duration. 
In the theory chapter 2.3, the action of gain and loss as well as the spectral filtering (gain dispersion) were presented as perturbations to the nonlinear Schrödinger equation 2.14. In fact, they cause the soliton pulse to loose and shed energy into a socalled "continuum" [250, 251], which manifests itself in a small contribution to the solution of the master equation 2.22 and is not directly coupled to the soliton [252]. As indicated in figure 4.3 (a), this lost energy is initially confined to a background pulse of low intensity, which is too weak to experience significant nonlinear effects like SPM. While the soliton pulse itself is shaped by a balance between negative GDD and SPM (see section 2.2), the dispersion is not simulatenously balanced for the continuum. Therefore, it is temporally spread into regions where the loss already slowly recovers. The resulting "cleanup" of the continuum radiation due to the group delay mismatch will eventually stabilize the soliton as long as its losses are smaller than the continuum loss.

(a)

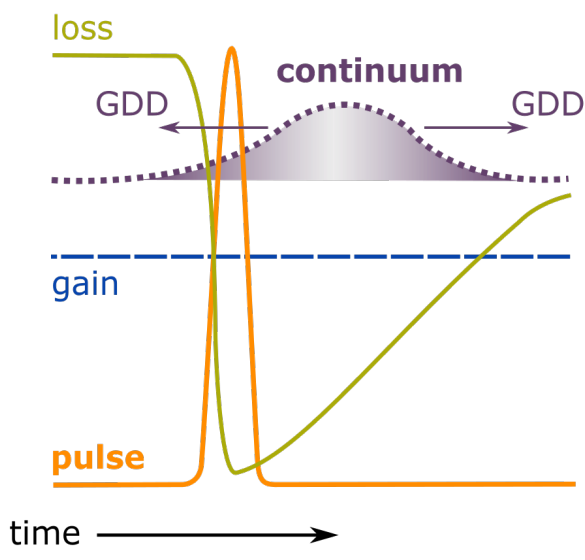

(b)

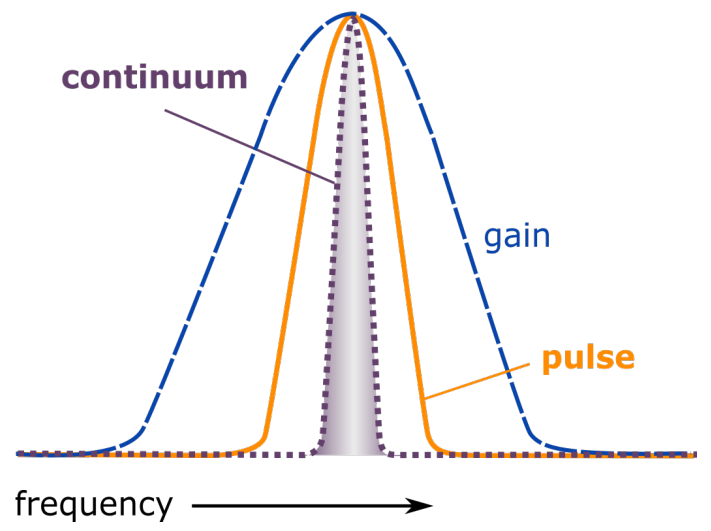

Fig. 4.3: Soliton mode-locking in (a) the temporal and (b) the spectral domain accompanied by the appearance of a background radiation. As illustrated in (a), a mode-locked laser with strong soliton-like pulse shaping is characterized by a slow absorber action that leaves a long net gain window open behind the main pulse. Even though it might allow a background radiation to grow, the intra-cavity GDD causes the initially weak continuum to spread in time, thus experiencing increasing losses. (b) On the other hand, the spectrum of the continuum is significantly narrower than that of the ultrashort soliton pulse. Hence, the spectral filtering action of the gain medium results in a gain advantage for the narrowband continuum. The figure is adapted from [250].

When evaluating the continuum formation in the frequency domain, however, it reveals a gain advantage of the narrower background radiation when compared to a broadband soliton pulse (see figure $4.3(\mathrm{~b})$ ). This is related to the limited gain bandwidth and the spectral filtering action of the gain medium. The same is true for pulses with a longer pulse duration and smaller spectrum. Therefore, when a laser cavity is operated at very high pulse energies or decreased amount of 
negative dispersion, a single pulse can split its energy into two or even more pulses, having individually much longer pulse durations than the original one $\left(E_{p} \propto 1 / \tau_{0}\right.$, see section 2.2.3) [253, 254]. To prevent the continuum from reaching threshold and avoid the onset of multi-pulsing, as was recorded in figure 4.4 (b), a large modulation depth of the saturable absorber can be helpful to maintain stable soliton mode-locking. Also, it can add additional loss for the growing continuum [250]. In this respect, large values of the Kerr strength are seeked after for KLM-based oscillators.

(a)

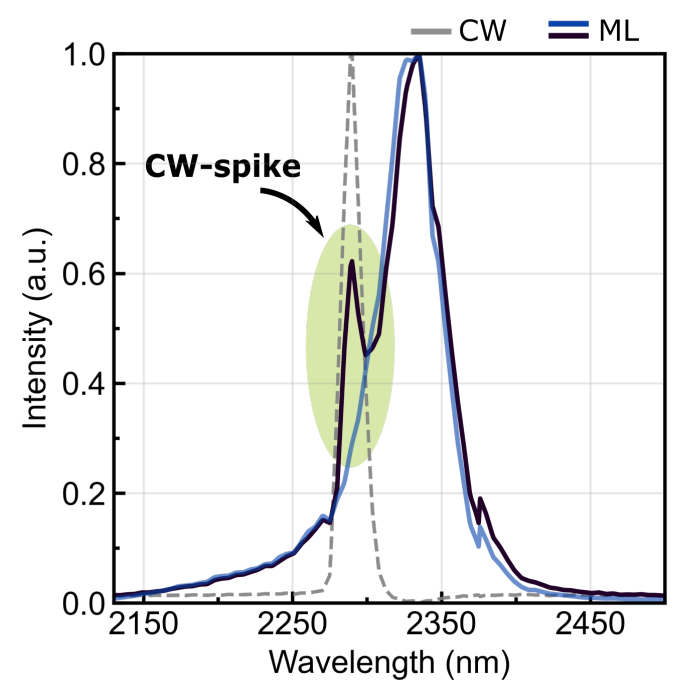

(b)
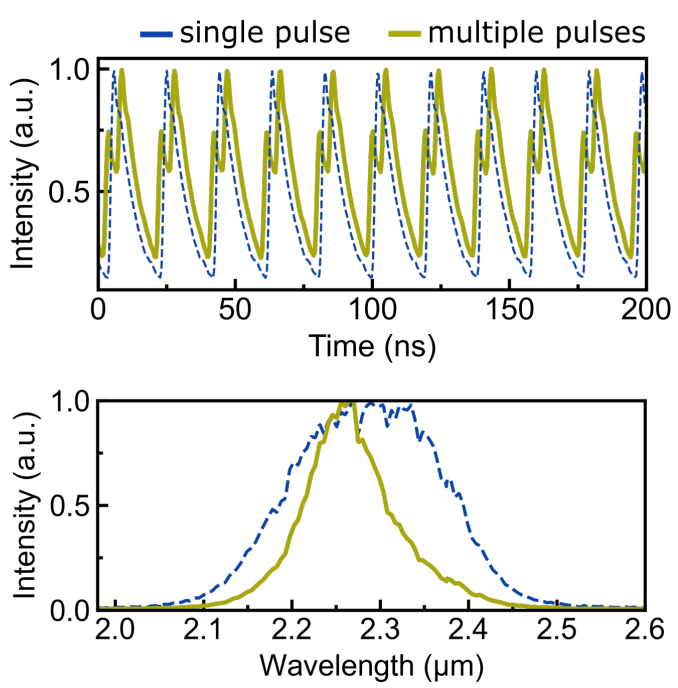

Fig. 4.4: Recorded mode-locking instabilities in the form of (a) a continuous-wave background and (b) multi-pulsing. The data was taken during the buildup and subsequent optimization phase of MIRanda2. As indicated in (a), a CW-breakthrough (dark blue) was observed in mode-locked operation, and could be removed by a decrease in pump power or a shift in gain medium position (light blue). Moreover, the $\mathrm{CW}$-spike position coincides with the spectrum in continuous-wave operation (dashed grey line), which is attributed to the wavelength at the maximum absorption cross section of the Cr:ZnS crystal. In figure (b), multi-pulsing was captured with a fast photodetector (Thorlabs, DET10D2), while a simultaneous reduction in pulse bandwidth was observed in the spectral domain - attributed to the longer pulse durations. It should be noted that the spectra were recorded with the same grating-based and fiber-coupled spectrometer as in figure 3.13, being only of limited spectral resolution. Hence, the fringe-like interference pattern in the mode-locked spectrum, which is typically observed for multi-pulsing, could not be resolved here.

The second diode-pumped oscillator presented in this chapter is meant to operate at higher power levels and shorter pulse durations than before, rendering the cavity more susceptible to the aforementioned instabilities. Hence, a closer investigation of the $\gamma_{K e r r}$-parameter was performed via nonlinear ABCD-matrix simulations 
to identify potentially favorable operation conditions and guidelines for the experimental fine adjustment of the new cavity.

As defined in equation 2.8, the Kerr strength is a measure of the small signal relative spot size variation and denotes the change in cavity mode size due to a small increase in the intra-cavity peak power. For example, if the Kerr strength is positive/negative, the mode diameter increases/decreases in mode-locked laser operation. Moreover, the magnitude of $\gamma_{K e r r}$ can be evaluated as a function of the distance $z$ between the two focusing mirrors as well as for varying crystal positions. Because Gaussian laser beams of non-vanishing peak power experience a lensing effect inside nonlinear media such as the gain crystal, one needs to derive nonlinear ABCD-matrices that account for the peak-power-dependent self-focusing. In this respect, a useful approach is presented in [104, 255], in which the crystal is divided into many thin Kerr-lens slices, whose thickness $l_{c r}$ is chosen small enough to ensure a negligible change in beam size per plate. The crystal can then be written as a stack of thin-lens matrices, where each matrix $M_{\text {slice }}$ can be represented as a superposition of a thin-lens effect with nonlinear focal length $f_{K e r r}$ (equation 2.7) and propagation through a single slice of thickness $l_{c r}$ with refractive index $n_{c r}$ [104]:

$$
M_{\text {slice }}=\left(\begin{array}{cc}
1 & 0 \\
-\frac{1}{f_{K e r r}(\omega)} & 1
\end{array}\right)\left(\begin{array}{cc}
1 & l_{c r} / n_{c r} \\
0 & 1
\end{array}\right) .
$$

This definition is similar to the one presented by Magni et al. [103, 256], however, with the difference that they split the crystal into two blocks of equal length. The Kerr nonlinearity is then grouped into one single matrix, which is surrounded by the linear propagation segments inside the crystal blocks.

To numerically evaluate the Kerr strength over the cavities' two stability zones, the following procedure is applied: first, after setting up the system's ABCD round-trip matrix, the beam size $w$ at each Kerr-lens plate is calculated for zero intra-cavity peak power $\left(P_{0}=0\right)$ according to $[88,225]$ :

$$
q=\frac{A q+B}{C q+D}
$$

Here, $A, B, C$ and $D$ denote the ABCD matrix elements for a complete cavity round-trip and $q$ refers to the beam parameter defined as $q^{-1}=R^{-1}-i \lambda /\left(\pi w^{2}\right)$ with the $\left(1 / \mathrm{e}^{2}\right)$ Gaussian beam radius $w$ and the wavefront curvature $R$. From this intitial set of beam sizes, the ABCD-matrices for the Kerr slices can be determined at a certain intra-cavity power level $\left(P_{0}>0\right)$, resulting in a new round-trip matrix for the system. With equation 4.2, the new set of mode sizes is then derived at each Kerr-lens plate again. Next, the ABCD-matrices for the Kerr slices are calculated 
again for $P_{0}>0$, with the new mode diameters arising from the corresponding system matrix. This step is repeated in an iterative manner for the same power $P_{0}>0$ until a steady state is reached; at this point, the beam sizes at the Kerr-lens plates effectively do not change anymore, and equation 2.8 can be written as a difference equation to extract the Kerr strengths for a certain normalized power $p=P_{0} / P_{c r}[257]:$

$$
\gamma_{\text {Kerr }}=\frac{1}{p} \frac{\omega(p)-\omega(0)}{\omega(0)}
$$

For soft-aperture KLM, the $\gamma_{K e r r}$-parameter was averaged over the crystal to include contributions from the whole crystal region [255]. By contrast, the Kerr strength analysis in hard-aperture KLM would rather focus on a certain position inside the cavity, which is typically chosen to be the position of the mechanical slit close to one of the end-mirrors [258, 259]. The same was also true for the simulation presented in the theoretical section 2.1.2.

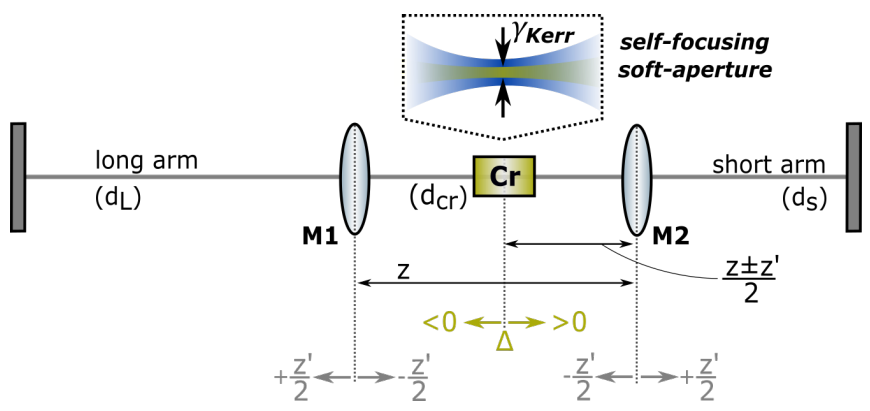

Fig. 4.5: Representative model for the nonlinear ABCDmatrix calculations, with $z$ : fixed distance between the focusing optics, $z^{\prime}$ : variation of $\mathrm{z}, d_{c r}$ : crystal length, $d_{l / s}$ : cavity arm lengths, $\Delta$ : deviation of the crystal location from the center position.

\begin{tabular}{l|c} 
& MIRanda2 \\
\hline $\mathrm{Cr}$ & Cr:ZnS \\
$d_{c r}$ & $5 \mathrm{~mm}$ \\
$n$ & 2.26 \\
$n_{2}$ & $100 \cdot 10^{-16} \mathrm{~cm}^{2} / \mathrm{W}$ \\
$\Delta$ & $\pm 10 \mathrm{~mm}$ \\
\hline$f_{M 1 / 2}$ & $100 \mathrm{~mm}$ \\
$\nu_{r e p}$ & $50 \mathrm{MHz}$ \\
$d_{s} / d_{l}$ & 0.5 \\
$z$ & $212.5 \mathrm{~mm}$ \\
$z^{\prime}$ & $\pm 10 \mathrm{~mm}$
\end{tabular}

Table 4.1: Simulation parameters, with $f_{M 1 / 2}$ : focal length of mirror $\mathrm{M} 1 / 2$.

For the simulations performed in this chapter, beam astigmatism was neglected and the curved cavity mirrors were modeled as thin lenses $\left(\mathrm{AOI}=0^{\circ}\right)$, resulting in equal Kerr strengths for the tangential and sagittal plane. A schematic representation of the simulation parameters as well as an overview of the respective values can be found in figure 4.5 and table 4.1. Following the procedure described above, a 2D-plot of the Kerr strength behaviour over the two stability zones was simulated for $p=0.0001$ and plotted in figure 4.6. Here, negative/positive values indicate a decrease/increase in beam size for $P_{0}>0$, while zero values refer to either unstable cavity configurations or positions of insignificant change in mode diameter. 
Also, two additional simulation runs were performed to further investigate the effect of different arm length ratios $d_{s} / d_{l}$ on the Kerr strength. The simulations were conducted for $d_{s} / d_{l}=0.3$ and 0.8 and are compared with the results for $d_{s} / d_{l}=0.5$ in figure 4.7, where all other parameters, including the repetition rate and step size, were kept constant for better comparability. Moreover, the spot size in the crystal was confirmed to stay almost unaffected at $\varnothing \approx 85-90 \mu \mathrm{m}$ for $P_{0}=0$ and different arm ratios.

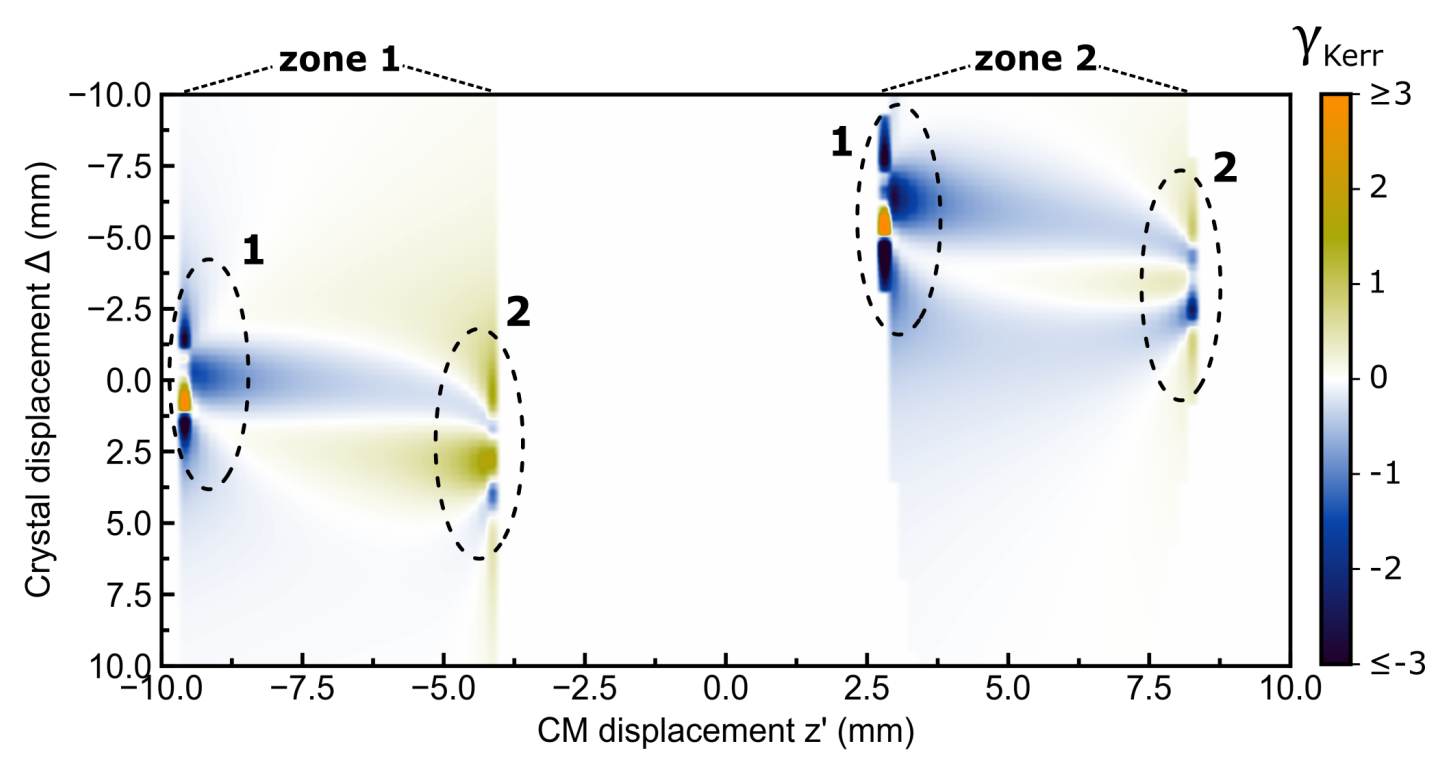

Fig. 4.6: Two-dimensional representation of the Kerr strength for varying crystal and curved mirror (CM) displacements. In the plot, two stable regions can be identified, which represent the two stability zones of the oscillator. Also, a noticeable enhancement of $\gamma_{K e r r}$ is apparent at the zone boundaries, with the respective edge positions being marked with dashed lines. When the crystal is exactly centered between the two focusing lenses, the displacement is $\Delta=0$.

Having a closer look at the 2D-maps, some general conclusions can be drawn:

- To achieve large variations in spot size and obtain strong nonlinear loss modulations, the cavity needs to be operated close to one of the stability zone boundaries (indicated with dashed circles). However, a large magnitude of the Kerr strength needs to be traded off against reduced cavity stability and laser gain. This observation is similar to the findings presented in [102-104, 255].

- For soft-aperture KLM, the simulation predicts operation at the first edge of stability zone 1 and zone 2 to be most favorable - assuming that the beam size for $P_{0}>0$ needs to decrease for stable mode-locking (see blue-shaded areas with $\left.\gamma_{\text {Kerr }}<0\right)$. The corresponding enhancement of $\gamma_{K e r r}$, however, seems to 
be more confined to the very edge in the first zone, while the conditions in the second zone appear to be slightly more relaxed. This renders the second stability zone more promising for efficient KLM, as also found in [102, 255].

- Investigating the Kerr strength with respect to crystal displacement reveals a strong positional dependence. Also, the direction of crystal movement seems to matter since $\Delta<0$ implies the crystal to be moved towards the longer arm, while $\Delta>0$ indicates a position more closely located to the shorter arm of the cavity.

- When varying the arm length ratio $d_{s} / d_{l}$ at a fixed repetition rate, the simulations predict a slightly increased nonlinear loss modulation for the higher ratios. Similar conclusions were also drawn in [260] for nearly symmetric resonators, which might even allow for self-starting operation [261].

(a)

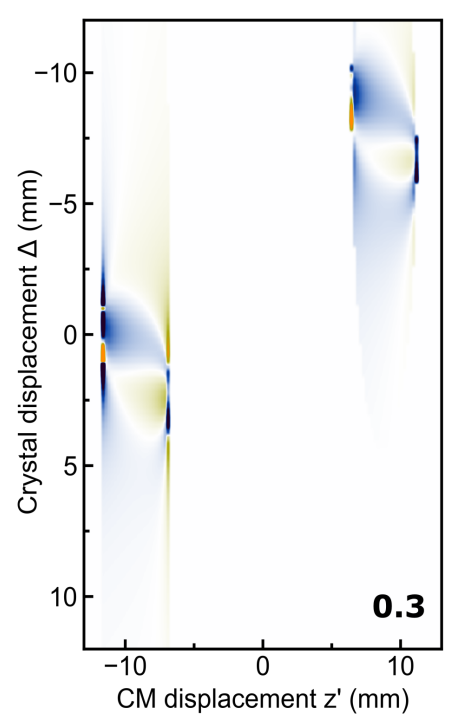

(b)

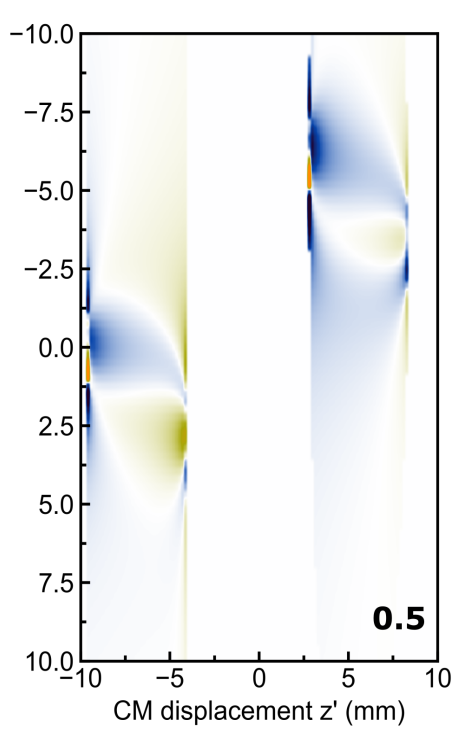

(c)

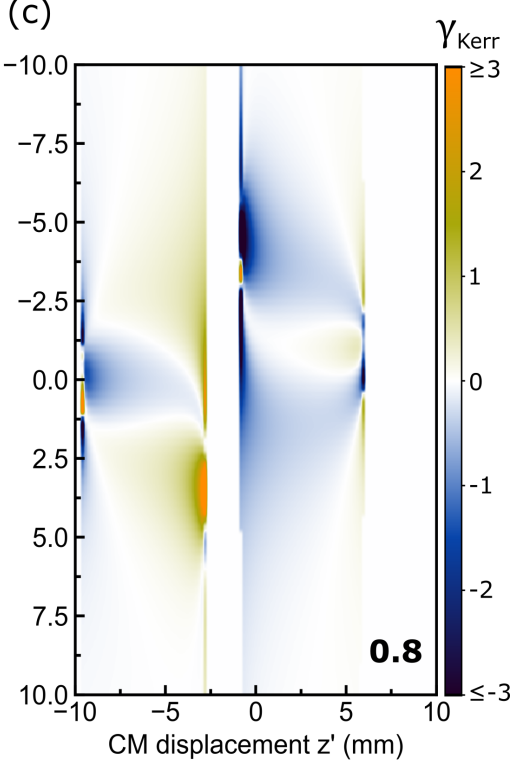

Fig. 4.7: Kerr strength simulations for varying arm length ratios, with $d_{s} / d_{l}=0.3$ (a), 0.5 (b), and 0.8 (c). The step size was kept constant for all calculations, indicating a slight increase in nonlinear loss modulation for converging arm lengths.

The small signal simulations performed here serve to illustrate some general laser characteristics in pulsed operation when operating within different stability zone regions. In reality, however, additional factors will influence the lasing behaviour of the cavity, making it significantly harder to give accurate predictions and run simulations. As shown in [262], for example, the stable regions will get continuously deformed when increasing the intra-cavity peak power, while thermal distortions arising from the comparably large quantum defect in $\mathrm{Cr}: \mathrm{ZnS} / \mathrm{ZnSe}$ gain media are likely to appear at the higher pump powers. Therefore, strong nonlinear effects in 
the gain crystal as well as thermal lensing effects might require the crystal to be shifted further away from the focal region than predicted by simulations. Moreover, the saturable absorber action for soft-aperture KLM might get less efficient beyond a certain power level, as was shown in figure 2.9, and is greatly affected by the mode-matching between the pump and cavity mode. If a crystal with a non-uniform transverse gain profile is used as a gain medium, also gain guiding effects would need to be taken into account [263].

\subsection{Comparison and Improvements over MIRanda1}

In this section, the experimental realization of a more powerful diode-pumped $\mathrm{Cr}: Z n S$ system will be presented, starting with an overview of the new pump setup and oscillator design. For KLM operation and its subsequent optimization, a clear strategy will be reported in section 4.2 .2 , based on the results achieved with MIRanda1 and the conclusions drawn from Kerr strength simulations.

\subsubsection{Pump and Cavity Design}

As introduced in section 4.1.1, polarization-multiplexing of two single-emitter laser diodes was applied. While this scheme appears straightforward to be implemented, the combination of two separate pump units adds complexity, while increasing the demand on mechanical stability and compactness. Therefore, a new pump assembly was realized, uniting all collimation lenses and the pump diode in a single optical cage system (see picture 4.8 (a)). Also, a new cooling architecture based on direct water-cooling was successfully adopted, rendering thermoelectric cooling of the diode base obsolete. To ensure a constant cooling temperature of $12^{\circ} \mathrm{C}$, the setup was equipped with an external circulating bath unit (Thermo Fisher Scientific, NESLAB RTE-7), which is specified for temperature stabilities of $\pm 0.01^{\circ} \mathrm{C}$ at a maximum cooling capacity of $500 \mathrm{~W}$ at $20^{\circ} \mathrm{C}$. As a result, a long term measurement of the direct laser diode output revealed a stable performance with an emitted power of 4 watts (figure 4.8 (a)).

Similar to MIRanda1, the slow and fast axis emission of the diodes was manipulated with a set of aspheric and cylindrical lenses to achieve a focal spot diameter of around 90-95 $\mathrm{\mu m}$ for the fast axis. This number was obtained through linear ABCD-matrix analysis, when simulating a laser cavity that was envisaged to operate at a repetition rate of $50 \mathrm{MHz}$ and be equipped with curved mirrors of $200 \mathrm{~mm}$ focal length. For the first diode assembly, the same C-Mount type was used as before (SemiNex Corp., C-132), however, with the $400 \mathrm{~mm}$ cylindrical lens being replaced by a pair of lenses $\left(\mathrm{C} 1^{*}, \mathrm{C} 2^{*}\right)$. This allowed to expand and re-collimate the diode's 
slow axis emission in a more compact fashion (see figure 4.8 (b)). For the second pump unit, a lensed version of the diode (SemiNex Corp., C-132-118) was tested, whose highly-divergent fast axis was already collimated to $10 \mathrm{mrad}$ with a $\mathrm{f}=274 \mathrm{\mu m}$ lens. Hence, a single cylindrical lens $\left(\mathrm{C} 4^{*}\right)$ was needed to collimate the slow axis, while a cylindrical lens telescope (C3, C5) expanded the collimated beam diameter of the fast axis. Both diodes were operated at supply currents around $12 \mathrm{~A}$, resulting in $2 \cdot 2.5 \mathrm{~W}$ of average power when measured in front of the pump lens (L). Despite all optics being AR-coated for the pump wavelength, about $10 \%$ of total power were lost in the lens collimation systems, while an additional $6 \%$ of the s-polarized diode beam were reflected at the $45^{\circ}$-input coupler mirror. Finally, a diameter of 85-90 $\mathrm{mm}$ and 170-190 $\mathrm{\mu m}$ was determined for the fast and slow axis in the focus, being in good agreement with the envisaged spot sizes and the spot size ratio of 1:2 (fast:slow axis).

(a)
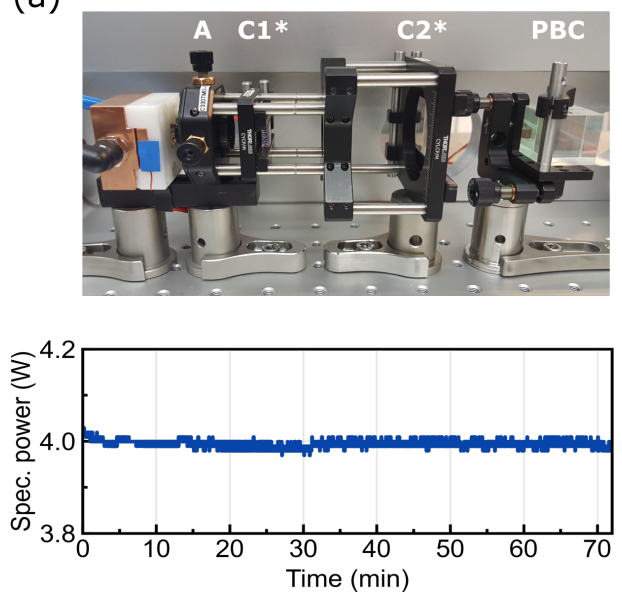

(b)
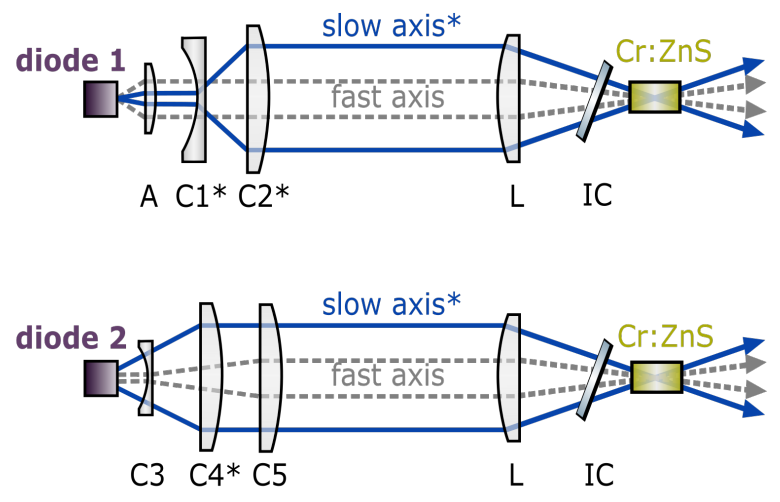

Fig. 4.8: Performance of the new diode mount and schematic of the two pump collimation assemblies. (a) Picture of the pump unit, with the diode mount being attached to a lens cage system. Also, direct water-cooling of the diode base was implemented, resulting in stable output power operation as shown in the figure below. PBC: polarizing beam combiner. (b) Schematic of the lens combinations used to address the slow and fast axis emission of diode 1 and 2, respectively. A: aspheric lens $(\mathrm{f}=3.10 \mathrm{~mm}), \mathrm{C}$ : cylindrical lenses $\left(\mathrm{f}_{\mathrm{C} 1^{*}}=-15 \mathrm{~mm}, \mathrm{f}_{\mathrm{C} 2^{*}}=100 \mathrm{~mm}\right.$, $\left.\mathrm{f}_{\mathrm{C} 3}=-25 \mathrm{~mm}, \mathrm{f}_{\mathrm{C} 4^{*}}=70 \mathrm{~mm}, \mathrm{f}_{\mathrm{C} 5}=150 \mathrm{~mm}\right), \mathrm{L}$ : achromatic pump lens $(\mathrm{f}=100 \mathrm{~mm})$, IC: input coupler. Asterisks $\left(^{*}\right)$ denote lenses that were used to address the slow axis only.

For the laser oscillator, a $5 \mathrm{~mm}$ long AR-coated polycrystalline gain medium (IPG Photonics) with a clear aperture of $1.9 \mathrm{~mm} \cdot 4 \mathrm{~mm}$ was used and placed inside a $\mathrm{X}$-fold cavity at normal-incidence angle. To avoid thermal instabilities arising from the increased heat load at higher pump powers, Cr:ZnS was preferred over Cr:ZnSe, which has an about 1.5 times higher thermo-optic coefficient (see table 3.1). At a doping concentration of $3.5 \cdot 10^{18} \mathrm{~cm}^{-3}$, the single-pass absorption was measured to be $\sim 81 \%$, with the water-cooled copper heat sink being connected to the same 
circulating chiller as the diodes. To further improve the system's performance and minimize the pump beam astigmatism arising from the $45^{\circ}$-input coupler mirror, the thickness of the latter was reduced from $6 \mathrm{~mm}$ to $3 \mathrm{~mm}$, while the incident angle on the curved mirrors was kept low at $\sim 2-3^{\circ}$. In contrast to MIRanda1, also the pump setup could be incorporated into the monolithic chamber, resulting in a higher stability and a footprint of only $60 \mathrm{~cm}$ x $60 \mathrm{~cm}$ for the entire system.

(a)

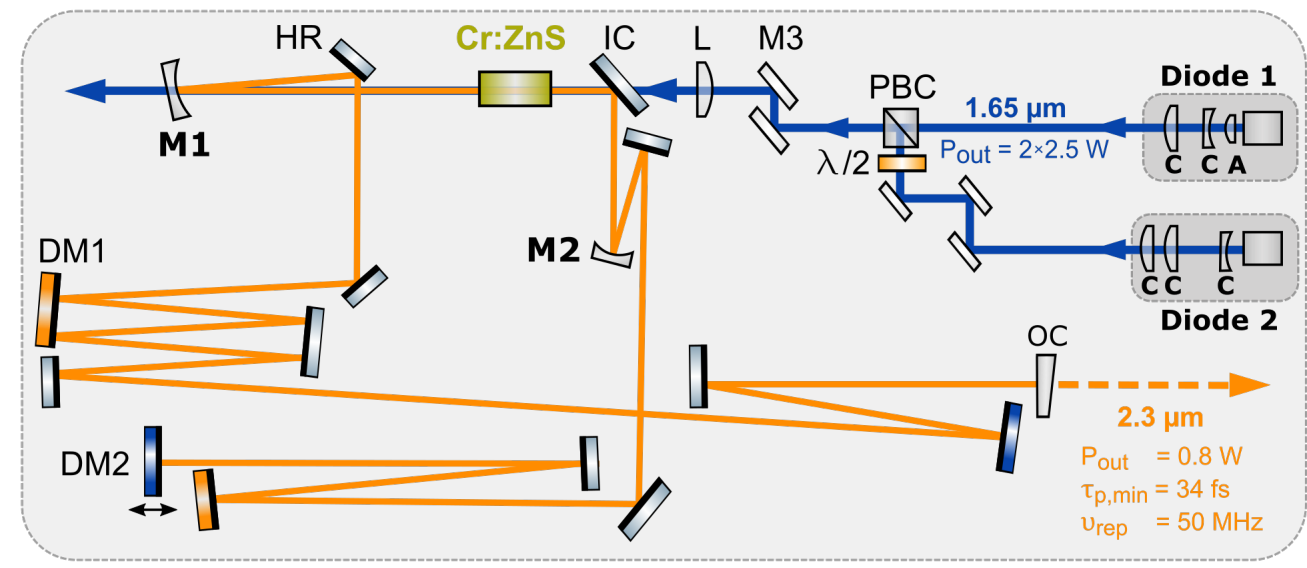

(b)

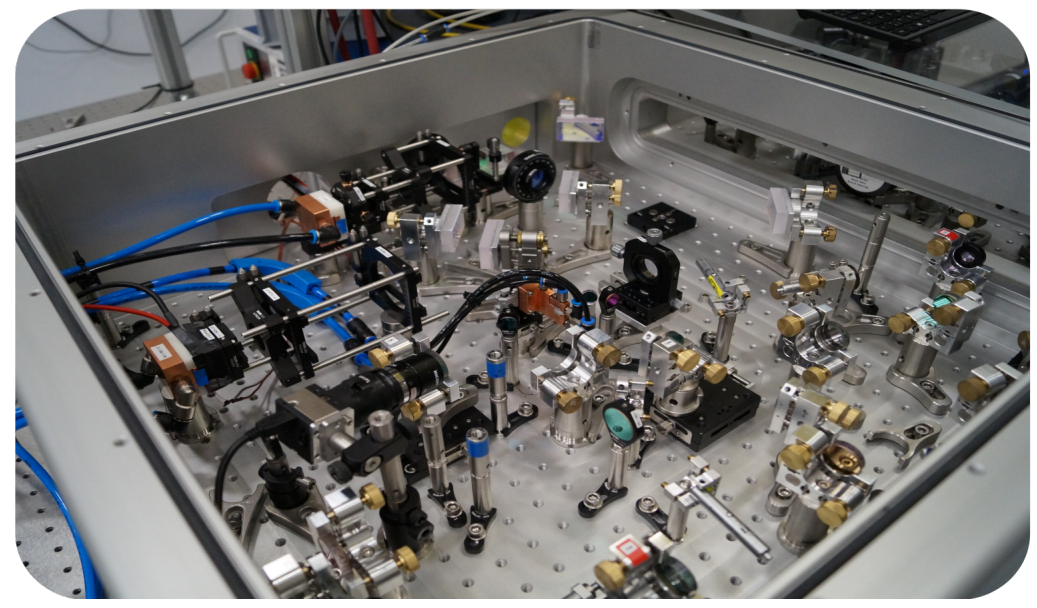

Fig. 4.9: (a) Experimental setup of MIRanda2 with (b) a picture of the laser system. Diode 1: Type C-132, Diode 2: Type C-132-118, C: cylindrical lens, A: aspheric lens, PBC: polarizing beam combiner, $\lambda / 2$ : half-wave plate, M3: highly-reflective rectangularshaped mirrors (1.4-1.7 $\mu \mathrm{m})$, L: achromatic pump lens $(\mathrm{f}=100 \mathrm{~mm})$, IC: input coupler, $\mathrm{M} 1$ and $\mathrm{M} 2: \mathrm{ROC}=-200 \mathrm{~mm}, \mathrm{DM} 1$ and DM2 (orange/blue): dispersive mirrors, HR (grey): highly-reflective mirrors optimized for 2.0-2.6 $\mu \mathrm{m}, \mathrm{Cr}$ :ZnS: gain medium $(5 \mathrm{~mm})$, OC: $25 \%$ output coupler. Mode-locked operation was started by a fast translation of the end-mirror. 


\subsubsection{Mode-Locked Performance}

To initiate and further optimize Kerr-lens mode-locked operation, a clear strategy was pursued: First, the cavity parameters were kept similar to the ones of $M I$ Randa1, given its successful realization of few-cycle pulse generation. Therefore, only one laser diode (C-132) was operated initially, and an arm length ratio of 0.7 was used for the cavity design. Also, to start soft-aperture KLM, the resonator was adjusted to operate at the 2 nd edge of stability zone 1, with the crystal being shifted towards the shorter arm. This agrees well with the simulation results shown in figures 4.6 and 4.7, indicating high relative magnitudes of the Kerr strength for crystal displacements $\Delta>0$, very similar to the design procedure described in [215].

Once pulsed operation was initiated by a slight detuning and rapid movement of the end miror, the crystal and curved mirror positions were fine-adjusted to enable reliable and stable mode-locked operation. The latter could be further improved through an optimization of the pump focus location, with the pump lens being positioned on a mechanical translation stage. As a next step, the pump and intra-cavity power was increased with the aid of the second laser diode (C-132-118) However, this resulted in mode-locked spectra being accompanied by a pronounced CW-background or being affected by the onset of multi-pulsing (see figures 4.4). Ways to cope with these kind of instabilities are to shift the gain medium out of focus or to reduce the pump power. While a stronger misalignment of the cavities' end-mirror can also help to maintain clean single-pulse operation, they inevitably reduce, at fixed output coupling (OC) ratio, the intra-cavity peak power and the average output power of the oscillator.

In the experiment, the OC ratio was increased step-wise starting at $11 \%$, with the achieved mode-locked output power and corresponding intra-cavity power being depicted in figure 4.10. Even though the output power was improved by a factor of three, the second pump diode was at no time operated at its full capacity due to the appearance of a CW-spike at higher powers. This was rather suprising, given the very high output coupling ratios of up to $64 \%$, which is - to the best of the authors knowledge - the largest reported for Cr:ZnS/ZnSe lasers so far [64]. Also, one would expect that the output coupling rate attains an optimum value, beyond which the output power decreases for a given pump rate [264, 265]. This optimum can be explained by the fact that the output power tends to increase for higher OC transmission, but also tends to decrease beyond a certain value due to the reduced number of cavity photons at increasing cavity losses. Experimentally, however, it was not possible to clearly identify the point of alteration at which the highest mode-locked output power can be achieved. Instead, when using OC ratios larger than $64 \%$, the oscillator was not mode-lockable anymore. 


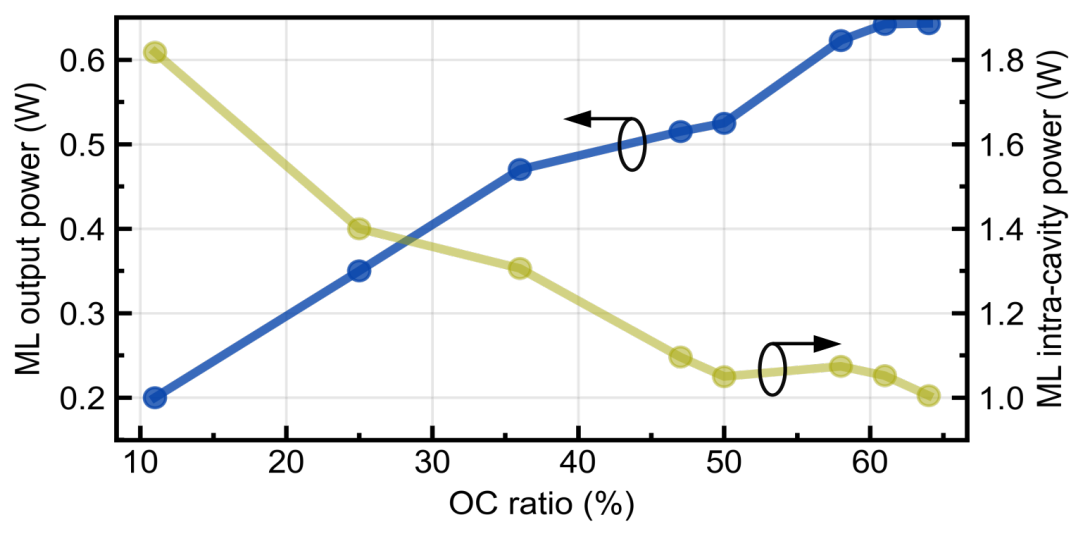

Fig. 4.10: Increase of the output coupling ratio in mode-locked operation. By combining several output couplers $(3 \%, 11 \%, 14 \%, 25 \%)$, very high rates of up to $64 \%$ could be achieved. The intra-cavity power levels were deduced from the measured output powers in mode-locked operation. It should be noted that the intra-cavity dispersion and thus the generated pulse durations and peak power levels slightly changed when different output couplers were inserted. Also, the power values were recorded for clean single-pulse operation without $\mathrm{CW}$-spike.

To investigate whether the strong tendency for CW-spiking and multi-pulsing is caused by the low brightness of the pump source, an Erbium fiber laser (Bktel, HPFL-370-1567-FCAPC) was installed to provide a diffraction-limited pump beam quality $\left(M^{2} \approx 1\right)$. Interestingly, no change in behaviour was observed upon modelocking. Hence, further strengthening of the self-amplitude modulation action in the gain medium was expected to be necessary for maintaining single-pulse operation at the higher pump levels. Since simulations predict soft-aperture KLM to be more favorable at the inner boundary of stability zone 2 (see figure 4.6), the cavitie's stability behaviour had to be characterized first. When measuring the $\mathrm{CW}$ ouput power at varying curved mirror distances, as plotted in figure 4.11 (a), a suitable position for KLM was identified in the second zone. Also, it was experimentally confirmed to result in highly reliable mode-locked operation - even without the need for a shift in crystal position or misalignment of the cavity. The latter might be due to the increased SAM action inside the Cr:ZnS crystal, while the former result was already predicted by the simulations (see figure 4.6): when increasing the distance between the curved mirrors by about $7-8 \mathrm{~mm}$ to move the cavity operation away from the second edge in stability zone 1 to the first edge in stability zone 2, the crystal displacement $\Delta$ amounts to a very similar value of around $7 \mathrm{~mm}$. Therefore, no strong change in crystal position is expected, which was also confirmed experimentally.

Though the CW-spiking turned out to be much weaker in the 2nd stability zone, further improvement was needed. Because the arm length ratio was shown to 
have some impact on the Kerr strength (see figure 4.7 ), different $d_{s} / d_{l}$-values were tested. The results are plotted in graph 4.11 (b) and reveal an ideal ratio of 0.5 for extracting the highest power in single-pulse operation. Mode-locked output powers of up to $1 \mathrm{~W}$ were finally measured at the full pump power and without the onset of pulse instabilities.

(a)

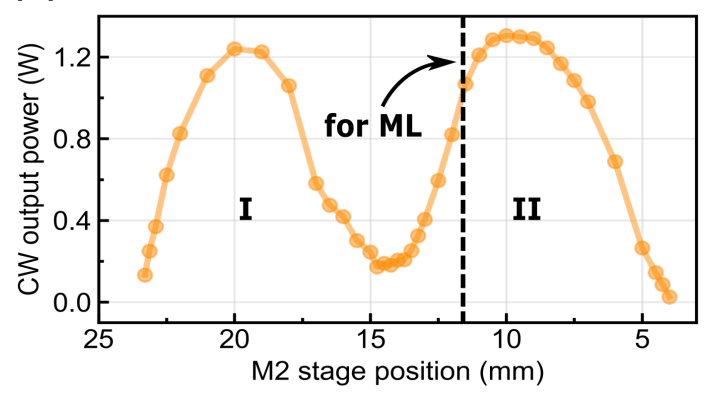

(b)

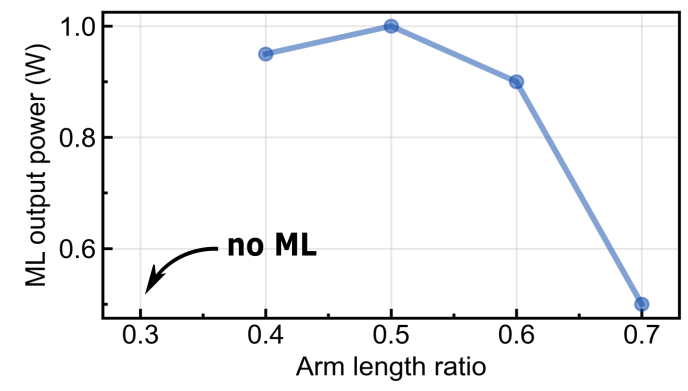

Fig. 4.11: (a) Stability zone scan and (b) KLM performance at varying arm ratios $d_{s} / d_{l}$ for an output coupling rate of $25 \%$ and a repetition rate of $50 \mathrm{MHz}$. In (a) decreasing values on the x-axis correspond to a shift of the curved mirror M2 away from the crystal.

(a)

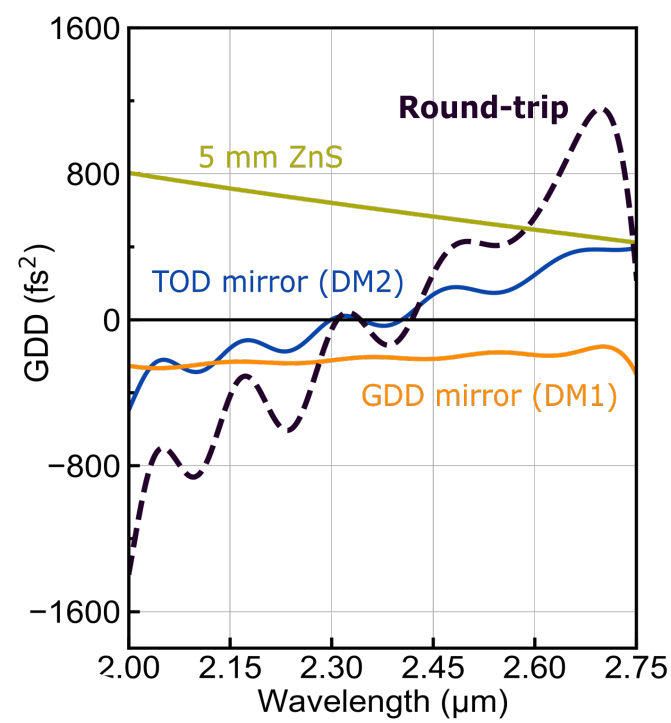

(b)
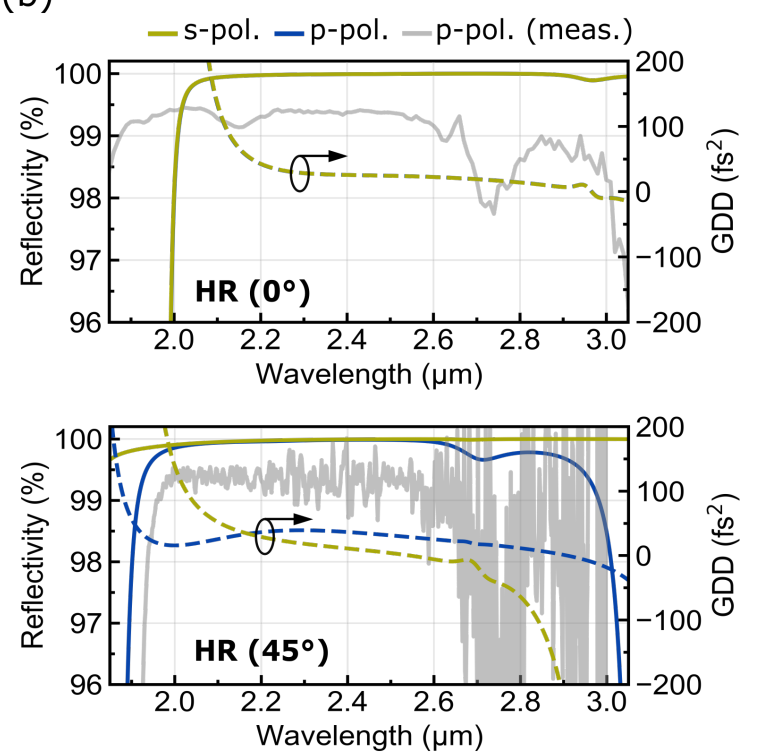

Fig. 4.12: (a) Theoretical dispersion values of the $5 \mathrm{~mm} \mathrm{Cr:ZnS} \mathrm{gain} \mathrm{medium} \mathrm{as} \mathrm{well} \mathrm{as} \mathrm{the}$ dispersion arising from a single GDD (DM1) and TOD (DM2) mirror, which was also used for MIRanda1. Also, the total round-trip dispersion is shown for the combination of mirrors that experimentally resulted in the shortes pulse duration. (b) Theoretical reflectivity and dispersion curves for the two sets of highly-reflective (HR) mirrors, being designed for $\mathrm{AOI}=0^{\circ}$ or $\mathrm{AOI}=45^{\circ}$. For the $\mathrm{AOI}=0^{\circ}$ case, the s-polarized and p-polarized curves are equivalent. Also, the measured reflectivity for p-polarized light is added. 
After having optimized the mode-locked output power, the intra-cavity dispersion was fine-tuned to further improve the output pulse duration. For the secondgeneration system, a new set of dispersive mirrors (DM1) was tested, being designed to compensate for the GDD and TOD of the $5 \mathrm{~mm}$ long Cr:ZnS crystal. In theory, 4 bounces on this mirror fully balance the gain medium's dispersion while adding slight negative dispersion of GDD $\approx-250 \mathrm{fs}^{2}$ for soliton mode-locking. Similar to the optimization steps performed for MIRanda1, different combinations of mirrors were tested in a systematic manner, revealing the optimal configuration to be 6 bounces on DM1 and 3 bounces on the TOD mirror DM2 per round-trip. This resulted in the shortest output pulse durations for an output coupling ratio of $25 \%$, with the corresponding round-trip dispersion being calculated and plotted in figure 4.12. Also, it seems that other cavity optics like the output coupler or HR mirrors have caused extra dispersion, explaining the need for additional TOD mirrors (DM2) and the discrepancy with the theoretical design.

(a)

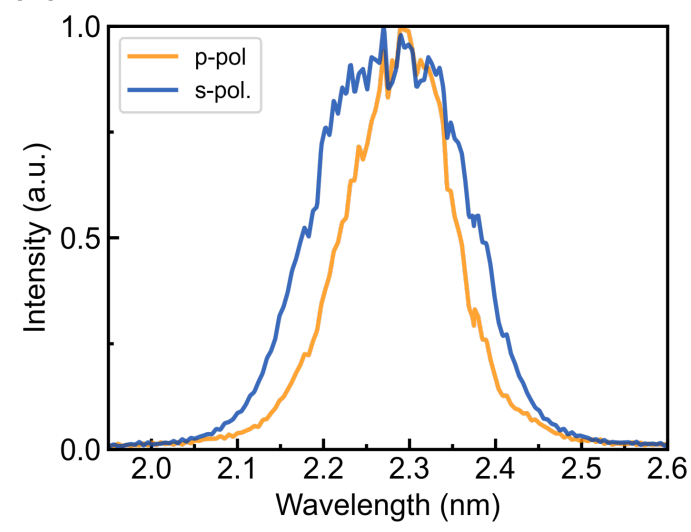

(b)

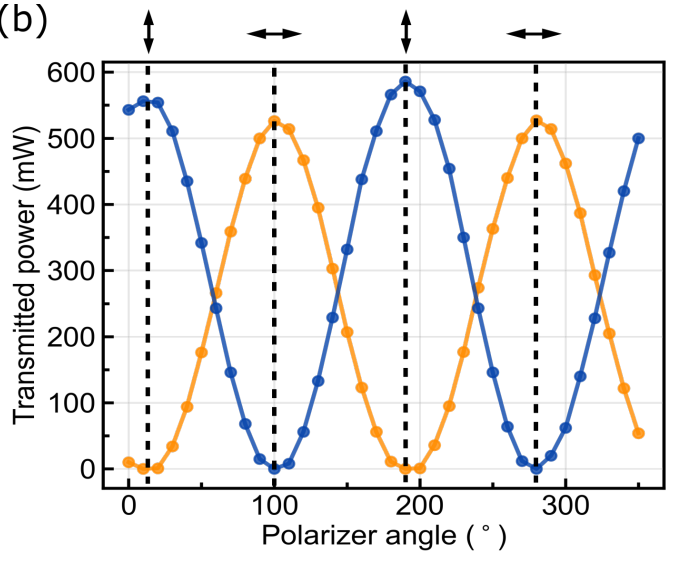

Fig. 4.13: Observed jump in the ML output polarization when measured at constant cavity parameters. Corresponding measurements of the output spectrum and polarization are shown in (a) and (b), respectively. The polarization was determined via measuring the transmitted power through a wideband IR polarizer (Innovation Photonics, PGC-5).

Since no intra-cavity element was placed at Brewster's angle in order to avoid further addition of material dispersion, the oscillator's polarization was not fixed. Therefore, for a given combination of cavity mirrors, the output pulses were measured to be either purely p- or s-polarized upon ML operation. Respective measurements are depicted in figure 4.13, which reveal a slight change in output spectral bandwidth for different polarization states. This behaviour is attributed to the highly-reflective coating of the $45^{\circ}$-mirrors, revealing very different dispersion characteristics and an improved reflectivity for s-polarized light (figure 4.12 (b)). As will be seen in chapter 5 , these optics were therefore not considered for the third-generation system. 
(a)
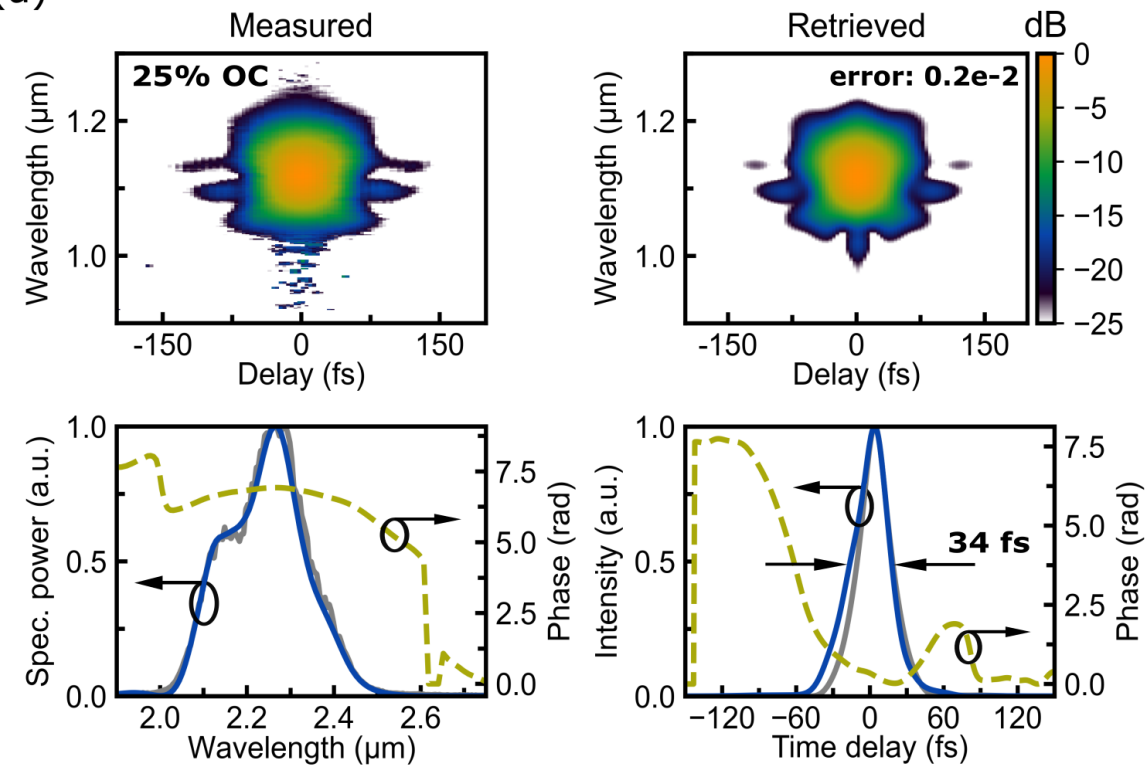

(b)
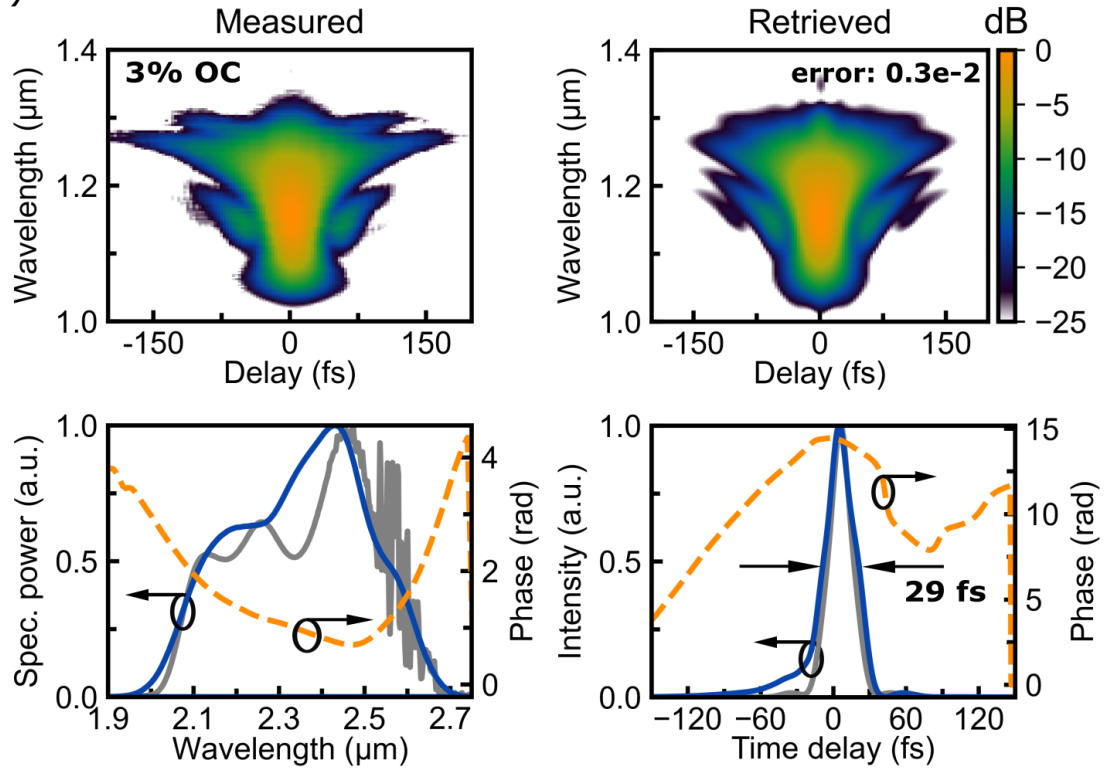

Fig. 4.14: (a) SHG-FROG measurement of the output pulses at a $25 \%$ output coupling ratio. (b) For comparison, another FROG trace was taken at a very low OC rate of $3 \%$ to illustrate the effect of higher intra-cavity power on the generated pulse duration. Both FROG traces were retrieved on a grid size of 512.512 points, with the measured spectra and calculated FTL intensity profiles indicated as grey lines. 
In its final state, the oscillator was providing stable s-polarized output pulses with pulse durations as short as 34 fs (Fourier-transform limit: $32 \mathrm{fs}$ ), corresponding to nearly four optical cycles. Combined with mode-locked output powers of over $800 \mathrm{~mW}$ at a $25 \%$ OC ratio and at a repetition rate of $49.9 \mathrm{MHz}$, this laser system delivered almost three-times higher peak powers compared to the first KLM diode-pumped $\mathrm{Cr}^{2+}$-doped II-VI laser presented in chapter 3 . In the continuous-wave regime, about $1.2 \mathrm{~W}$ of average power were achieved when operating at the center of the stability zones. Interestingly, a $5-10 \%$ drop in power was observed when the laser switched to pulsed operation. According to numerical simulations presented in reference [102], this drop might occur when the cavity is operated at higher Kerr nonlinearities. In this case, $\mathrm{CW}$ oscillations experience increased residual gain.

A respective SHG-FROG measurement is depicted in figure 4.14 (a), and was taken without further post-compression of the output pulses. Since the $25 \%$ output coupling mirror was made of $3 \mathrm{~mm} \mathrm{BaF}_{2}$, it caused only very little dispersion when compared to other commonly used materials (see table 2.2). For frequency doubling, a $20 \mu \mathrm{m}$ thick GaSe crystal was used, since the transmission of BBO starts to drop in an unsmooth fashion beyond $2 \mu \mathrm{m}$, which will likely cause artifacts in few-cycle pulse measurements. To also illustrate the effect of higher intra-cavity power on the pulse spectral bandwidth, another FROG measurement was performed at a very low output coupling rate of only $3 \%$ (see figure $4.14(\mathrm{~b})$ ). After accounting for the additional dispersion of the OC mirror (fused silica, $6 \mathrm{~mm}$ ), a very broad spectrum could be measured at an average power of about $150 \mathrm{~mW}$. It resulted in a retrieved pulse duration of $29 \mathrm{fs}$ with a Fourier-transform limit of $27 \mathrm{fs}$. The shape of the FROG trace, however, displays a change in phase in the longer wavelength range. This is potentially due to the cavity being operated at very low negative dispersion. In this regime, spectral oscillations in the round-trip dispersion, which are caused by mirror dispersion or air absorption lines around 2.5-2.7 $\mu \mathrm{m}$, can result in slight crossings of the zero-dispersion line, hence disturbing clean soliton mode-locking in the negative GDD regime.

To fully characterize the performance of the laser system, similar to MIRanda1, the mode-locked output power stability was measured and depicted for a 3.5 hours snapshot in figure 4.15 (a). With corresponding RMS power fluctuations of less than $0.24 \%$, the oscillator was operating reliably for the whole working day and with a clean single-mode output beam profile. In addition, the horizontal and vertical beam quality factor $M^{2}$ of the linearly polarized mode-locked output pulse trains were proven to be nearly diffraction limited with $M^{2}<1.1$ (figure 4.15 (b)). Since the oscillator was pumped by two laser diodes simultaneously, the slope efficiency for CW and ML operation was measured for decreasing pump powers of the second diode (C-132-118), revealing high efficiencies of $62 \%$ and 70\%, respectively (figure 4.15 (c)). 
(a)

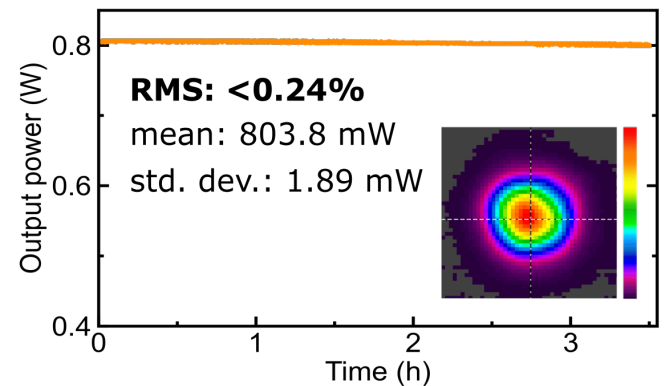

(b)
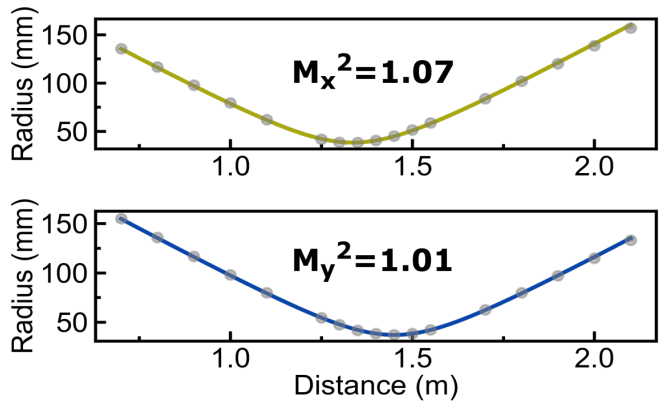

(c)
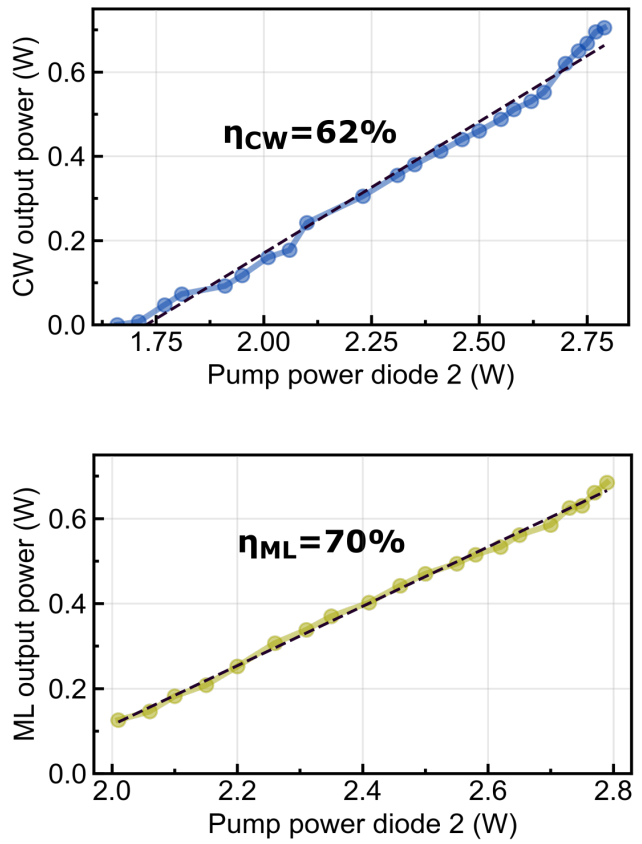

Fig. 4.15: Output performance of MIRanda2. (a) Mode-locked output power stability recorded with the thermal power meter (Coherent, PS19) and corresponding single-mode beam profile. (b) $M^{2}$-measurement of the horizontal and vertical beam axis of the mode-locked output. (c) Slope efficiency curves measured for a decrease in pump current of the second diode.

\section{Amplitude noise measurements}

In the measurements presented in the previous chapter, the directly diode-pumped oscillator exhibited a surprisingly low noise peformance, which was hardly detectable when measured at the fundamental output wavelengths. Instead, a workaround via second-harmonic generation in a PPLN crystal was needed to enhance the oscillator's output noise. To determine the noise characteristics of the second-generation Cr:ZnS system, however, the measurement setup was equipped with a new wideband voltage amplifier (VA), rendering analysis of the direct laser output feasible.

The setup is sketched in figure 4.16 (a), and includes the same RF spectrum analyzer and photodetector (PD) as used in section 3.3.3. To enlarge the signal-to-noise ratio (SNR) between the oscillator's low-frequency noise and the background noise of the measurement devices, the detector signal was sent through a low-noise and AC-coupled variable gain VA, which was operated at the highest gain level of $60 \mathrm{~dB}$. Though its respective cut-off frequency was set to $10 \mathrm{MHz}$, the strong carrier frequency around $50 \mathrm{MHz}$ was filtered by an additional low-pass filter (LPF) placed in front. To plot the measured noise levels in logarithmic units of $\mathrm{dBc} / \mathrm{Hz}$ and evaluate 
the integrated RMS noise, the same formulae were applied as in the previous noise analysis, with the exception that the theoretically generated DC-voltage level ${ }^{1}$ of $U_{D C, 60 \mathrm{~dB}}=44 \mathrm{mV} \cdot 10^{60 / 20}=44 \mathrm{~V}$ was now taken as reference level in equation 3.6.

(a)

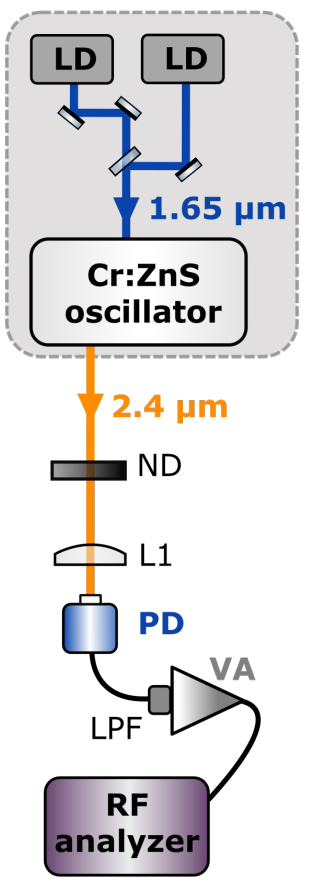

(b)
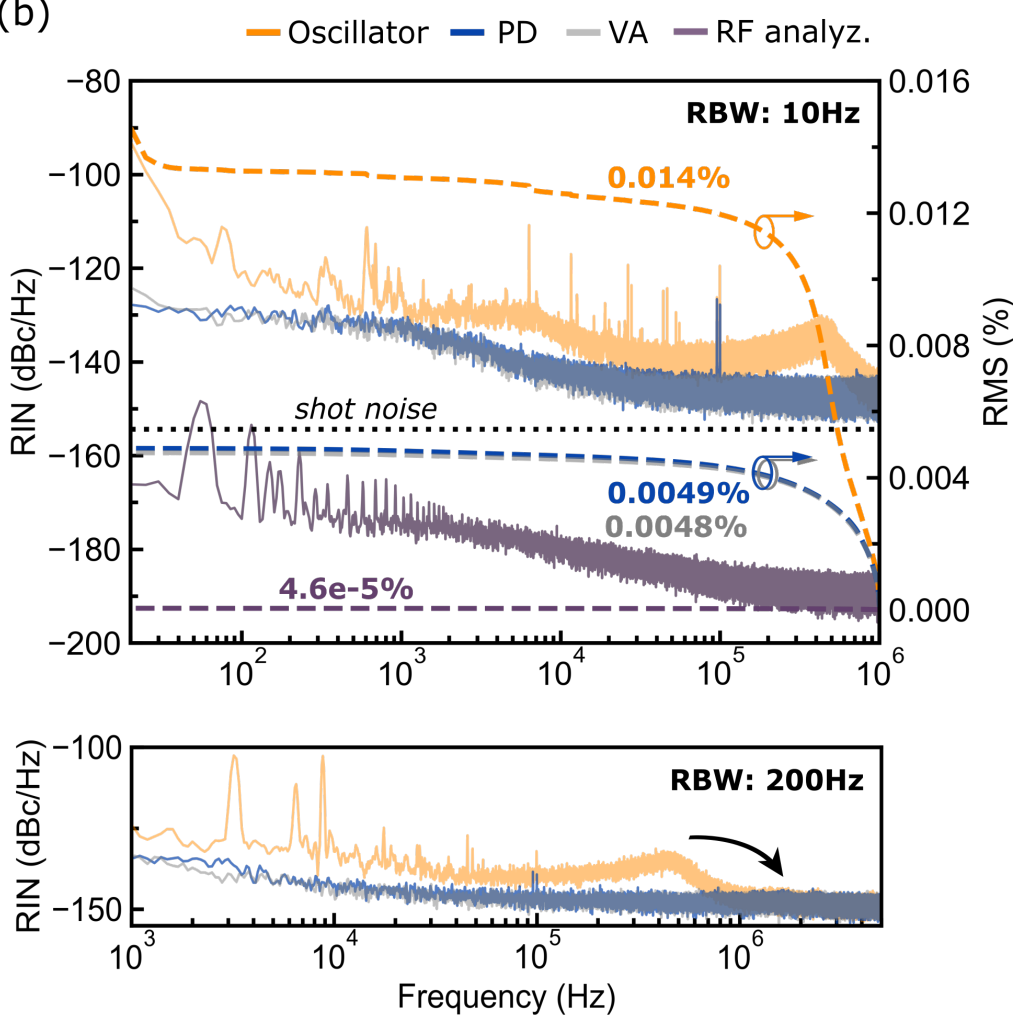

Fig. 4.16: Amplitude noise measurements and integrated RMS noise of the mode-locked output of MIRanda2. (a) Setup. LD: InP laser diode, ND: neutral density filter wheel, L1: N-BK7 lens ( $\mathrm{f}=25 \mathrm{~mm}$ ), PD: biased InGaAs photodetector (Thorlabs, DET10D2), LPF:

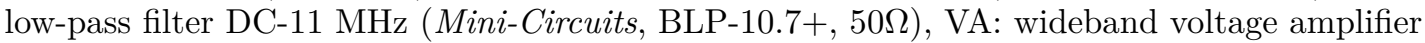
(FEMTO, DHPVA-101), RF analyzer: radio-frequency spectrum analyzer (Agilent, E4447A). (b) Measurements and integrated RMS noise of the direct mode-locked output (orange) along with data on instrument noise. Below, the RIN was measured in a broader span up to $5 \mathrm{MHz}$, and illustrates the drop in noise signal to the background noise floor at frequencies around $1 \mathrm{MHz}$.

The results are plotted in figure 4.16 (b) along with the corresponding background noise of the photodetector as well as the voltage amplifier and the spectrum analyzer. The latter two were obtained for signal inputs that had been terminated with a $50 \Omega$ resistor, while the noise floor of the PD was recorded when the optical signal to the detector was blocked. Obviously, the noise floor of the photodetector

1 To calculate the DC-voltage $U_{2}$ after amplification with a certain voltage gain factor $G_{V}$ [dB], the following formula was used: $U_{2}=U_{1} \cdot 10^{G_{V}} / 20$. 
was slightly lower than the noise floor of the VA, resulting in very similar integrated RMS noise values of $0.0049 \%$ and $0.0048 \%$. The shot noise level was also plotted and calculated according to equation 3.5, resulting in a value of $-154 \mathrm{dBc} / \mathrm{Hz}$.

Compared to the output characteristics of MIRanda1, a noise peak at frequencies around $400-600 \mathrm{kHz}$ is apparent, which is most likely related to slight relaxation oscillations of the Cr:ZnS gain crystal. The $f^{-1}$-behaviour at the very low frequencies together with the drop in noise signal around $1 \mathrm{MHz}$, however, is in accordance with the previous findings. Moreover, by using the new voltage amplifier, the SNR of the overall measurement system could be noticeably improved. With the background noise floor of the RF analyzer being located at $-190 \mathrm{dBc} / \mathrm{Hz}$ and the noise level of the VA and PD approaching $-148 \mathrm{dBc} / \mathrm{Hz}$ at the higher frequencies, the influence of instrument noise on the oscillator signal was minimized. For the frequency range between $20 \mathrm{~Hz}$ and $1 \mathrm{MHz}$, it resulted in a very low integrated RMS noise of $0.014 \%$ for the mode-locked output - comparable to reported [234] and even commercially available $^{2}$ ultrafast Ti:sapphire laser systems.

\subsection{Chapter Summary}

With MIRanda2, a powerful KLM Cr:ZnS oscillator pumped by two single-emitter laser diodes was demonstrated, delivering mode-locked average powers of over $800 \mathrm{~mW}$ combined with pulse durations down to nearly four optical cycles. Despite the highly elliptic collimated pump beam shapes and the higher intra-cavity peak powers, stable soft-aperture mode-locking performance was achieved in accordance with nonlinear ABCD-matrix simulations. Moreover, the low amplitude noise of the directly diode-pumped oscillator could be retained, revealing a measured integrated RIN $[20 \mathrm{~Hz}-1 \mathrm{MHz}$ ] of less than $0.014 \%$ RMS for the fundamental wavelengths. Compared to the first-generation system, an almost three-fold increase in peak power was achieved via polarization-multiplexing of two pump laser diodes (see table 4.2). In addition, the pulse duration was shortened further with the aid of newly designed dispersive cavity mirrors. This places the power performance of directly diode-pumped $\mathrm{Cr}^{2+}: \mathrm{ZnS} / \mathrm{ZnSe}$ oscillators on the same level as of state-of-theart fiber-laser-pumped versions [64], with the diode-pumped systems representing one of the most attractive driving sources for efficient mid-infrared generation via nonlinear $\chi^{(2)}$ optical processes.

2 The Ti:sapphire oscillator "Mai Tai" from Spectra-Physics has a specified noise of $<0.15 \%$ RMS for a $10 \mathrm{~Hz}$ to $10 \mathrm{MHz}$ bandwidth. For more information on the product, the reader is referred to the website: https://www.spectra-physics.com/products/ultrafast-lasers/mai-tai (25.10.2020). 


\begin{tabular}{l|cc}
\hline & MIRanda1 [209] & MIRanda2 [266] \\
\hline ML mechanism & Kerr-lens ML & Kerr-lens ML \\
Gain medium & Cr:ZnSe (5 mm) & Cr:ZnS (5 mm) \\
$P_{\text {pump }}$ & $3 \mathrm{~W}$ & $2 \times 2.5 \mathrm{~W}$ \\
$\lambda_{\text {pump }}$ & $1.65 \mu \mathrm{m}$ & $1.65 \mu \mathrm{m}$ \\
$P_{C W}$ & $700 \mathrm{~mW}$ & $1200 \mathrm{~mW}$ \\
$P_{M L}$ & $500 \mathrm{~mW}$ & $800 \mathrm{~mW}$ \\
$\tau_{0}$ & $45 \mathrm{fs}$ & $34 \mathrm{fs}$ \\
$\nu_{\text {rep }}$ & $64.7 \mathrm{MHz}$ & $49.9 \mathrm{MHz}$ \\
OC ratio & $34 \%$ & $25 \%$ \\
\hline$E_{0}$ & $\mathbf{7 . 7} \mathbf{~} \mathbf{~ J}$ & $\mathbf{1 6} \mathbf{~ n J}$ \\
$P_{0}$ & $\mathbf{1 5 1} \mathbf{~} \mathbf{~}$ & $\mathbf{4 1 5} \mathbf{~ W W}$ \\
\hline
\end{tabular}

Table 4.2: Comparison of the laser performance and the respective improvements achieved over the first directly diode-pumped $\mathrm{Cr}^{2+}$-doped II-VI laser system. 



\section{PUSHING THE PEAK POWER FOR DI- RECT MID-INFRARED GENERATION}

With MIRanda2, directly diode-pumped few-cycle $\mathrm{Cr}^{2+}$-doped II-VI lasers have now reached the power level of state-of-the-art fiber-laser-pumped versions, hence opening the door to nonlinear frequency conversion into the mid-IR driven by 2-3 $\mu \mathrm{m}$ lasers. Among the various techniques that can be exploited, two main categories are typically employed: nonlinear spectral broadening in waveguide structures [267-270] and parametric frequency conversion processes in nonlinear crystals [32, 35, 36, 271]. In recent years, both types have been successfully applied to Cr:ZnS/ZnSe pump systems $[30,33,34,73,167,272]$, resulting in 1.7-octave-spanning supercontinua (1.6-5.1 $\mathrm{\mu m})$ at $350 \mathrm{~mW}$ average power via step-index fluoride fibers [73]. To access even longer wavelengths, however, the technique of intra-pulse difference frequency generation (IDFG) holds great potential, and spectral components up to $18 \mu \mathrm{m}$ could already be demonstrated with the chromium-based driving lasers [33, 34, 167].

DFG is a nonlinear $\chi^{(2)}$ optical process and allows for a self-stabilization of the carrier-envelope phase (CEP) when two pulses sharing the same shot-to-shot CEP fluctuations mix together in a nonlinear medium [273, 274]. The carrierenvelope phase is an important property of ultrashort pulses ${ }^{1}$ and has a strong effect on the electric field waveform of the pulse, especially when going down to few-optical-cycle pulse durations. Moreover, emerging mid-infrared applications such as field-resolved infrared spectroscopy [39, 40] require the CEP of the laser pulse to be stable in time. Otherwise, when exciting molecular vibrations with pulsed laser light, the consecutive waveforms emitted by the sample can hardly be interpreted via electro-optic sampling (EOS), whose dynamic range increases with averaging and thus longer measurement time ( $\sim 10$ s of seconds [40]). For example, for a driving laser with a specified repetition rate of $28 \mathrm{MHz}, 28$ million electric-field waveforms are generated per second, and a multiple of them would need to be detected. If the waveform is not identical for every pulse, it is impossible to draw reliable conclusions from the measured EOS traces after averaging.

Without sophisticated feedback mechanisms acting on the pulses' phase, modelocked lasers generally fail to emit phase-stable pulses. In fact, laser intensity

1 The frequency components of a laser pulse can be written as $\nu_{m}=\nu_{C E O}+m \cdot \nu_{r e p}$, with $m$ denoting the number of the mode. A pulse is said to be CEP-stable when the carrier-envelope offset (CEO) frequency $\nu_{C E O}$ and the repetition rate $\nu_{r e p}$ are well controlled. 
variations, mechanical vibrations, temperature or air turbulences cause the CEP to fluctuate randomly from pulse to pulse [274]. For lasers generating soliton-like pulses, this time-dependent phase change $\delta \psi(t)$ can be expressed as [275]:

$$
\delta \psi(t)=\phi_{\text {Kerr }}\left(\frac{d P_{0}}{d W}\right)_{W_{0}} \cdot \delta W(t)-\omega_{0} D \delta \omega_{0}(t),
$$

with peak power $P_{0}$, pulse energy $W\left(W_{0}=W_{t=0}\right)$, center frequency $\omega_{0}$, net intracavity GDD $D$, and soliton phase shift $\phi_{\text {Kerr }}$ due to the optical Kerr effect in the gain medium. Hence, changes in the center frequency $\delta \omega_{0}(t)$ and pulse energy $\delta W(t)$ together with external noise sources perturbing the peak power $P_{0}$ govern the overall phase fluctuations. Consequently, the DFG scheme is highly sought after for delivering the phase-stabilized pulses needed in applications: when mixing two pulses with the same shot-to-shot CEP fluctuations in a nonlinear crystal, these fluctuations are automatically cancelled for the generated difference frequency components [38, 276].

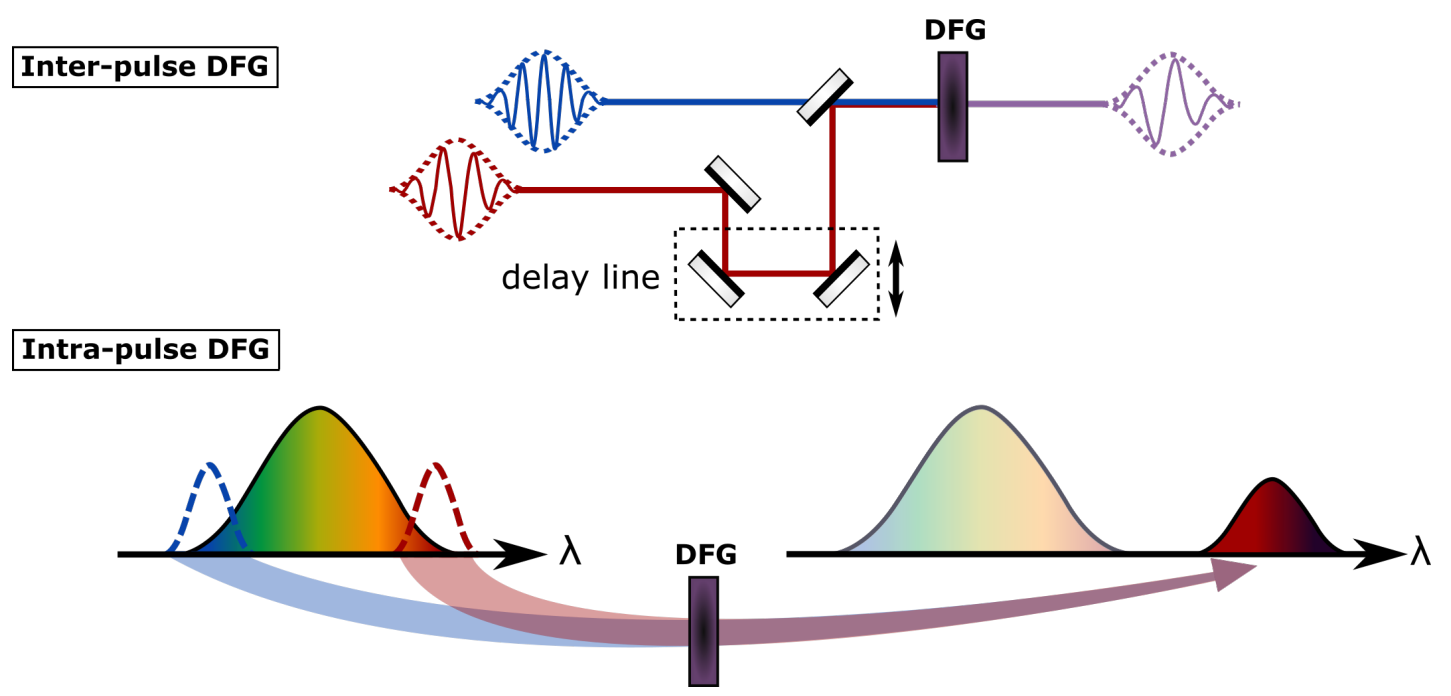

Fig. 5.1: Comparison of inter-pulse and intra-pulse difference frequency generation schemes. The figure is inspired by [276].

Compared to standard inter-pulse DFG, in which the interacting pump and seed beam originate from separate laser beams, intra-pulse DFG has become quite popular in this respect. Also referred to as optical rectification, its robust and compact single-beam geometry involves the mixing between different spectral components of the same ultrabroadband pulse (see figure 5.1). Without doubt, IDFG is experimentally much easier to set up and does not require precise spatial and temporal overlapping of two beams. Moreover, it is not affected by a timing jitter between the pump and seed pulse, which introduces additional fluctuations on the CEP. 
Regarding the nonlinear conversion efficiencies of DFG-based processes, however, they are only in the range of $10^{-3}-10^{-2}$ [277]. Even though efficiencies as high as $50 \%$ have already been demonstrated with spectral broadening in waveguides [73], the bulk materials used for DFG are far less sensitive to beam alignment and can often handle much higher power levels than waveguide structures. Thus, to also reach large signal strengths via DFG, a closer inspection of the scaling law of the generated MIR intensities $I_{3}$ is helpful [84, 277]:

$$
I_{3}(L)=\frac{1}{2} \epsilon_{0} c n_{3}\left|A_{3}(L)\right|^{2}=\frac{8 d_{e f f}^{2} \omega_{3}^{2} I_{1} I_{2}}{\epsilon_{0}^{3} c^{3} n_{1} n_{2} n_{3}} L^{2} \cdot \operatorname{sinc}^{2}\left(\frac{\Delta k L}{2}\right) .
$$

Here, $\Delta k=k_{1}-k_{2}-k_{3}$ is the momentum mismatch, which is a scalar in the case of IDFG, $L$ is the propagation distance in the crystal, $d_{e f f}=\frac{1}{2} \chi^{(2)}$ refers to the so-called effective nonlinear coefficient, $A$ denotes the amplitude of the opctical field, and $n$ is the frequency-dependent refractive index. The subscripts 1,2 and 3 relate to the pump, signal and generated DFG components with frequencies $w_{1}, w_{2}$ and $w_{3}$, respectively. When assuming perfect phase-matching conditions $(\Delta k=0)$ and solitonic pulses ( $\mathrm{sech}^{2}$-shape), the mid-IR intensity thus scales with the input pulse parameters as follows:

$$
I_{3} \propto I_{1} \cdot I_{2} \propto I_{0,1} \cdot I_{0,2} \quad \text { with } \quad I_{0}=0.88 \frac{P_{a v}}{\nu_{r e p} \tau_{p}} \frac{1}{A_{f o c}}=P_{0} \frac{1}{A_{f o c}} .
$$

Hence, to achieve large signal strengths, high peak intensities $P_{0}$ of the driving laser pulses are desired along with small focal spot sizes $A_{\text {foc }}$ in the nonlinear crystal $^{2}$. Recent demonstrations of Cr:ZnS/ZnSe-based IDFG involved further scaling of the average oscillator output power $P_{a v}$ to enhance $P_{0}$. By means of additional amplification stages, these master oscillator power amplifier (MOPA) systems can boost the average power level of the few-cycle laser pulses to several watts, albeit increasing the complexity of the overall setup [33, 34, 167].

In this chapter, a new approach is presented, in which IDFG is directly driven with the mode-locked output of a diode-pumped Cr:ZnS oscillator for the first time. As illustrated in figure 5.2, the simultaneous reduction in pulse duration $\tau_{p}$ and repetition rate $\nu_{r e p}$ also results in a peak power enhancement - potentially without the need for further amplification stages. Moreover, if the oscillator can be directly pumped with laser diodes, the low-noise characteristics of the mode-locked laser output are preserved and not affected by subsequent amplification.

\footnotetext{
2 It should be noted that the minimum focal spot size in the nonlinear optical crystal is limited by the damage threshold and the onset of multi-photon absorption at high peak intensities (see section 5.3.2).
} 


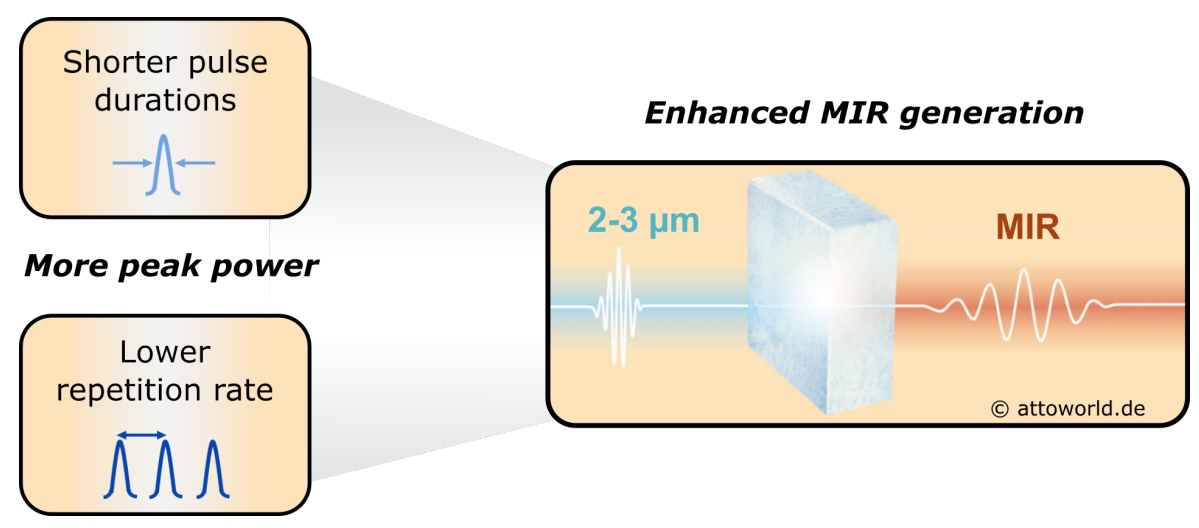

Fig. 5.2: Illustration of enhanced mid-infrared generation via shorter driving pulse durations and a lower repetition rate, denoting the operation characteristics of the third-generation laser system MIRanda3.

In addition, the shorter pump pulse duration also corresponds to a broader spectral bandwidth. Since the shortest mid-infrared wavelengths that can be generated upon IDFG are dictated by the frequency difference between the shortest $\left(\lambda_{1}\right)$ and longest $\left(\lambda_{2}\right)$ wavelength components of the driving laser beam $\left(\lambda_{3, \min }^{-1}=\lambda_{1}^{-1}-\lambda_{2}^{-1}\right)$, oscillators delivering broader output spectra and thus shorter pulse durations are highly desired. In table 5.1, four different pulse durations were considered, with the minimum achievable wavelength $\lambda_{3}$ being calculated for each case. It shows that shorter pulse durations allow to reach shorter and shorter DFG wavelengths, clearly illustrating the advantage of few-cycle laser oscillators to span ever increasing portions of the mid-infrared region.

\begin{tabular}{llc} 
Pulse duration & 20 dB-limits & $\boldsymbol{\lambda}_{\mathbf{3}, \boldsymbol{m i n}}$ \\
\hline$\tau_{p}=45 \mathrm{fs}$ & $\lambda_{1}=2100 \mathrm{~nm}$ & $12.6 \mu \mathrm{m}$ \\
& $\lambda_{2}=2520 \mathrm{~nm}$ & \\
\hline$\tau_{p}=34 \mathrm{fs}$ & $\lambda_{1}=2045 \mathrm{~nm}$ & $9.6 \mu \mathrm{m}$ \\
& $\lambda_{2}=2600 \mathrm{~nm}$ & \\
\hline$\tau_{p}=25 \mathrm{fs}$ & $\lambda_{1}=1960 \mathrm{~nm}$ & $7.0 \mu \mathrm{m}$ \\
& $\lambda_{2}=2720 \mathrm{~nm}$ & \\
\hline$\tau_{p}=15 \mathrm{fs}$ & $\lambda_{1}=1775 \mathrm{~nm}$ & $4.2 \mu \mathrm{m}$ \\
& $\lambda_{2}=3050 \mathrm{~nm}$ &
\end{tabular}

Table 5.1: Dependance of the shortest mid-IR wavelengths reachable for different driving pulse durations, with the first two $\tau_{p}$-values corresponding to MIRanda1 (45 fs) and MIRanda2 (34 fs). To obtain the shortest and longest wavelength components, the spectral limits at the $20 \mathrm{~dB}$ were considered for bandwidth-limited pulses. 
In the following, a new generation of diode-pumped few-cycle $\mathrm{Cr}^{2+}$-doped II-VI lasers is presented, operating at an unprecedented low repetition rate of $\nu_{\text {rep }}=23 \mathrm{MHz}$ combined with pulse durations measured down to three optical cycles. This unique combination significantly enhanced the output peak power of the oscillator, enabling direct generation of mid-infrared radiation via IDFG for the first time (section 5.3). However, further improvements in oscilllator and pump design (section 5.2.1) were needed to tackle challenges arising from higher intra-cavity peak power levels. Also, the effect of air dispersion on the mode-locked oscillator performance was studied by purging the chamber with dry nitrogen (see section 5.2.2).

\subsection{Challenges of Reaching Higher Peak Powers}

To operate the oscillator at higher peak powers $P_{0}$, the three laser parameters $P_{a v}, \nu_{r e p}$ and $\tau_{p}$ can be addressed (see equation 5.3). In principle, an increase in average power would be a viable approach. However, since the output power of a single-emitter InP pump laser diode is limited to 2.5 watts to 3 watts, an improvement over the second-generation system (chapter 4) would have only been achievable by incorporating additional pump diodes into the setup. Hence, to push the peak power from $400 \mathrm{~kW}$ to the $1 \mathrm{MW}$ level, the laser system would need to be operated at 2.5-times the average mode-locked power of MIRanda2, requiring at least two more pump diodes to be integrated via simultaneous polarization- and wavelength-multiplexing. Since this increases the complexity of the system, a better approach to peak power scaling is to lower the oscillator's repetition frequency. Though this approach is highly attractive, new challenges arise over the previous diode-pumped systems (chapter 3 and 4), which are briefly addressed in the following:

\section{- Enhanced nonlinearities:}

To scale the peak power of the passively mode-locked laser, one of the major challenges is attributed to the ever stronger nonlinear effects inside the gain medium. If they become excessive, multi-pulse instabilites will develop and affect the soft-aperture Kerr-lens mode-locked operation. While this was also observed for MIRanda2 (see section 4.1.2), the effect is expected to be even stronger in the third-generation system. Also, to reduce the nonlinearities, operation of the cavity in stability regimes with lower self-amplitude modulation and thus lower Kerr strength might be inevitable. However, this would come at the expense of a mode-locked operation being much more difficult to start. 
A useful measure to assess the strength of nonlinear effects is given by the so-called "B-integral" [88]:

$$
B=\frac{2 \pi}{\lambda} \int_{0}^{L} n_{2} I_{0}(z) d z
$$

with $n_{2}$ and $I_{0}(z)$ denoting the nonlinear refractive index and the optical peak intensity along the propagation direction $z$. To avoid serious distortion effects due to self-phase modulation or self-focusing in a nonlinear medium, the generally accepted criterion is to keep the cumulative B-integral below the value $\mathrm{B} \leq 3$ to 5 .

(a)

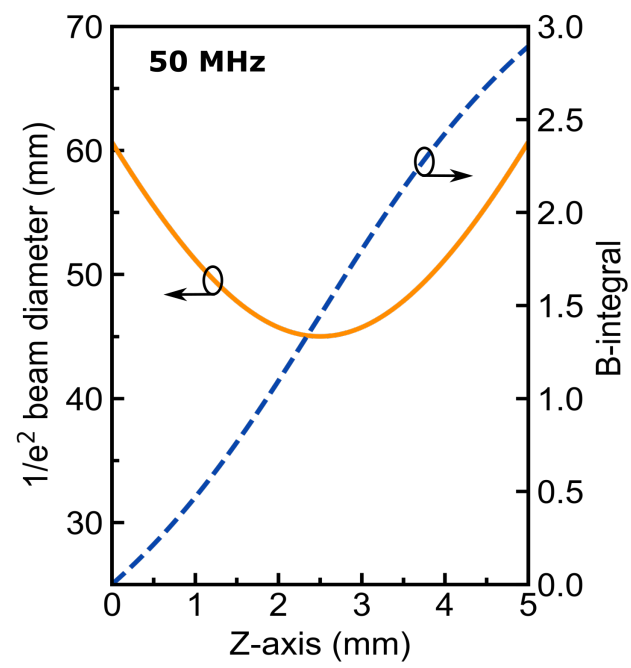

(b)

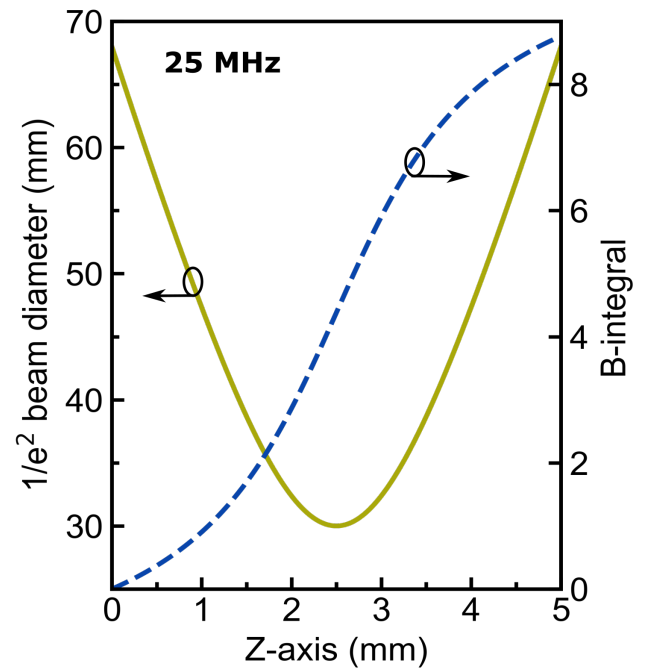

Fig. 5.3: Calculation of the single-pass B-integral inside the Cr:ZnS crystal $\left(n_{2}=1 \cdot 10^{-18} \mathrm{~m}^{2} / \mathrm{W}\right)$. The smaller the focal spot diameter, the higher the accumulated nonlinear phase shift (B-integral).

Considering the second-generation laser system presented in the previous chapter, mode-locked output parameters of $P_{a v}=800 \mathrm{~mW}$ and $\tau_{p}=34 \mathrm{fs}$ could be achieved at a repetition rate of $50 \mathrm{MHz}$ and an output coupling ratio of $25 \%$. With a focal spot diameter of $\sim 90 \mu \mathrm{m}$ and a central wavelength of $\lambda=2.3 \mathrm{\mu m}$, the B-integral for a single-pass through the $L=5 \mathrm{~mm}$ long $\mathrm{Cr}: Z n S$ gain crystal was calculated and plotted in figure 5.3 (a). The same parameters were also used for a calculation at half the laser repetition rate $(25 \mathrm{MHz})$. Assuming a simple lengthening of the cavity with the same cavity optics, and maintaining the arm length ratio of 0.5, ABCD-matrix analysis indicates the respective focal spot diameter in the crystal to be $\sim 60 \mu \mathrm{m}$. While the single-pass B-integral in MIRanda2 was still below $\mathrm{B}=3$, the decrease in 
repetition rate would result in $\mathrm{B}>8$ given the smaller focus size. Therefore, to avoid destabilization of the low repetition rate KLM oscillator, other approaches are required.

\section{- Low repetition rate design:}

The high cumulative B-integral of $>8$ at $25 \mathrm{MHz}$ repetition rate case calls for larger beam sizes in the crystal. A relatively simple way to achieve this is by selecting spherical mirrors with longer focal lengths. At the same time, however, limitations arise from mode-matching issues. As will be illustrated in section 5.2.1, the third-generation cavity design is meant to be based on a "classic" X-fold cavity rather than a "folded" resonator design (see figure 3.6 as a reminder). The classic design is much more elegant but requires the pump beam to be focused through one of the curved mirrors. Since the pump focusing lens needs to be placed in front of this spherical input coupler mirror, restrictions are imposed on the selection of the pump lens' focal length. Hence, to ensure a good mode-matching between the low-brightness pump beam and the fundamental cavity mode, the focal lengths of the spherical mirrors cannot be chosen arbitrarily long. Nevertheless, a suitable workaround could be found and will be presented in section 5.2.1.

\section{- Air dispersion:}

To enhance the peak power and improve the short-wavelength coverage upon IDFG, shorter durations of the driving laser pulses are of additional benefit besides the decreased repetition rate. However, when going towards ever shorter pulse durations, the spectral width of the laser will inevitably start to overlap with the water absorption region at around 2.6-2.8 $\mu \mathrm{m}$ (see figure 3.1). Since the laser was operated at ambient air conditions in a laboratory environment, the relative humidity $(\mathrm{RH})$ in the laser chamber amounted to about $25 \%$ in winter and $45 \%$ during summer for a room temperature of $\mathrm{T} \approx 20^{\circ} \mathrm{C}$. If the moisture content in the air cannot be reduced, soliton perturbation theory predicts strong spectral distortions of the travelling soliton due to the presence of water absorption lines [278]. This causes the refractive index and thus the dispersion of air to be modulated by sharp Fano-shaped features of varying strength and separation [279]. This additional dispersion severely affects the spectral shape of the laser pulse, and a different effect is observed for linear (a) and perturbed (b) soliton propagation (see figure 5.4). The former takes place for linear propagation, for example outside the resonator structure, resulting in spectral absorption dips as expected from the classic Lambert-Beer law ${ }^{3}$. When the soliton is already peturbed inside the laser

\footnotetext{
${ }^{3}$ It relates the intensity of the incident light $I_{0}$ to the intensity transmitted through an absorptive solution via $I=I_{0} \cdot 10^{-\epsilon c l}$, with the molar absorption coefficient $\epsilon$, the concentration $c$, and the optical path length $l[280]$.
} 
resonator, however, the pulse spectrum reveals sharp spectral bends with strongly suppressed absoprtion dips. Since the presence of absorption lines not only degrades the spatial but also the temporal pulse quality [279], the effect of water dispersion needs to be mitigated inside the laser chamber and will be analyzed in section 5.2.2.

(a)

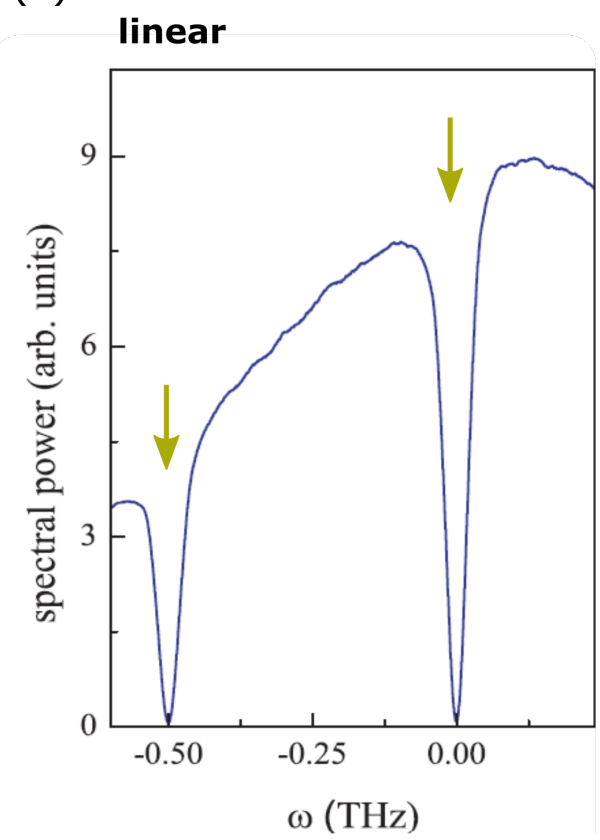

(b)

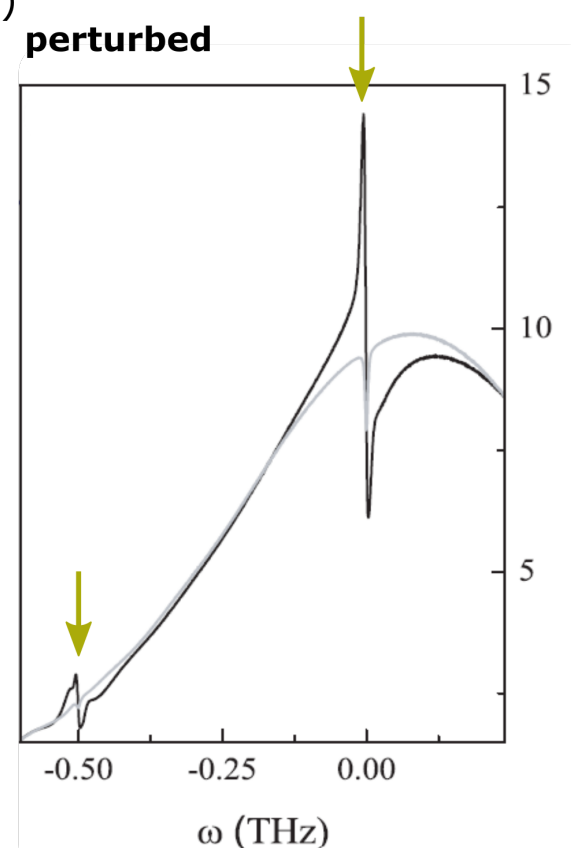

Fig. 5.4: Part of the laser pulse spectrum after linear propagation (a) and perturbed soliton propagation (b). The location of the two absorption lines is indicated with arrows. The data was taken from reference [278].

\subsection{Experimental Achievements}

To address the series of challenges listed above, the design of the laser system had to be modified. Moreover, when compared to the cavity design of the first- and second-generation system, no $45^{\circ}$-mirrors inside the oscillator were used to avoid limitations in their reflective and dispersive properties (see section 4.2.2). The resulting resonator layout was based on a classic bow-tie design, with all setup components assembled inside a single monolithic housing. The latter had a footprint of only $90 \mathrm{~cm}$ x $60 \mathrm{~cm}$ and enabled proper sealing of the laser assembly. Hence, studies on the effect of air dispersion on the mode-locked laser performance could be carried out through purging experiments (section 5.2.2). 


\subsubsection{Pump and Oscillator Setup}

To realize the classic bow-tie design but still allow for a good mode-matching between the pump and fundamental cavity beam, a workaround was needed. Certainly, one could choose the collimated pump beam diameter arbitrarily large such that any pump spot diameter can be achieved for a certain pump lens. For practical reasons, however, this is not ideal since large pump beam diameters require large pump optics, rendering the whole setup bulky and mechanically less stable. Instead, a solution was found as shown in figure 5.5: By combining two lenses with negative (L1) and positive (L2) focal lengths, the distance $d$ to the focus could be successfully increased. The reason behind is that the incident pump beam on the lens L2 was now divergent rather than collimated. To confirm that the focal spot diameters are still kept at a reasonably low level for this two-lens combination and do not get significantly distorted via spherical aberrations, measurements were performed and summarized in table 5.2. The focal lengths of the optics were chosen with regard to the new oscillator design, which should be based on a repetition rate of $25 \mathrm{MHz}$. With arm lengths of $1900 \mathrm{~mm}$ and $3800 \mathrm{~mm}$, spherical cavity mirrors with focal lengths of $\mathrm{f}=-200 \mathrm{~mm}$ and $\mathrm{f}=-300 \mathrm{~mm}$ were selected from the set of available cavity optics. According to ABCD-matrix analysis, this combination results in a focal spot diameter of $85-90 \mu \mathrm{m}$ for the fundamental cavity mode - comparable to MIRanda2.

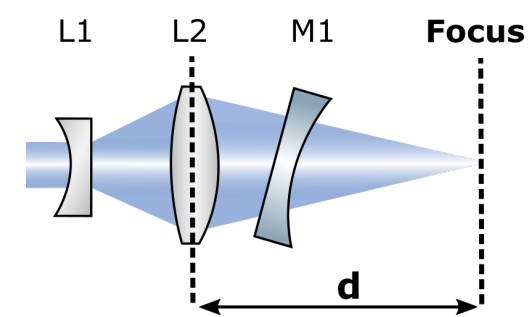

Fig. 5.5: Schematic of the twolens pump focusing unit. L1: $\mathrm{f}=-150 \mathrm{~mm}, \mathrm{~L} 2: \mathrm{f}=75 \mathrm{~mm}, \mathrm{M} 1$ : $\mathrm{ROC}=-200 \mathrm{~mm}$.

\begin{tabular}{l|cc} 
Optics & Focus $\varnothing$ & $\mathbf{d}$ \\
\hline L2 & $80 \mu \mathrm{m}(\mathrm{x})$ & $\mathbf{7 5} \mathbf{~ m m}$ \\
& $50 \mu \mathrm{m}(\mathrm{y})$ & \\
L2, M1 & $95 \mu \mathrm{m}(\mathrm{x})$ & $\mathbf{8 5} \mathbf{~ m m}$ \\
& $65 \mu \mathrm{m}(\mathrm{y})$ & \\
L2, M1, L1 & $140 \mu \mathrm{m}(\mathrm{x})$ & $\mathbf{1 3 5} \mathbf{~ m m}$ \\
& $95 \mu \mathrm{m}(\mathrm{y})$ &
\end{tabular}

Table 5.2: Measured focal spot diameters and distance $d$ for a given set of optics.

The measurement setup consisted of a meniscus lens with negative focal length as well as an achromat and a spherical mirror (see figure 5.5). This combination was experimentally found to provide the best performance regarding the minimum achievable focal spots. For the pump beam, the collimated output of a InP laser diode (SemiNex Corp., C-132-118) was used at a diode driving current of 10 A. The results are depicted in table 5.2 and reveal that the successive integration of the input coupler mirror (M1) and the defocusing lens (L1) into the measurement setup resulted in a 1.8-times increase in focal distance $(75 \mathrm{~mm} \rightarrow 135 \mathrm{~mm})$. At the same 
time, however, the focal spot diameters increased in the horizontal (x) and vertical (y) direction, which is attributed to the spherical aberrations imposed on the pump beam. Nevertheless, a tight spot diameter of $95 \mu \mathrm{m}$ was achieved for the fast axis direction, which is only marginally larger than the theoretical value for the cavity mode. Hence, a good mode-matching is still expected. Besides the tight focus, it is also the distance of $\mathrm{d}=135 \mathrm{~mm}$ that enables a successful realization of this new design. Since the spherical mirror M1 has a focal length of $100 \mathrm{~mm}$, the two-lens combination $(\mathrm{L} 1, \mathrm{~L} 2)$ can be safely placed in front of it because $d>f_{M 1}$. Finally, it should be mentioned that the $\mathrm{M}^{2}$-values of the laser diode beam were confirmed to be not seriously affected by this optics combination.

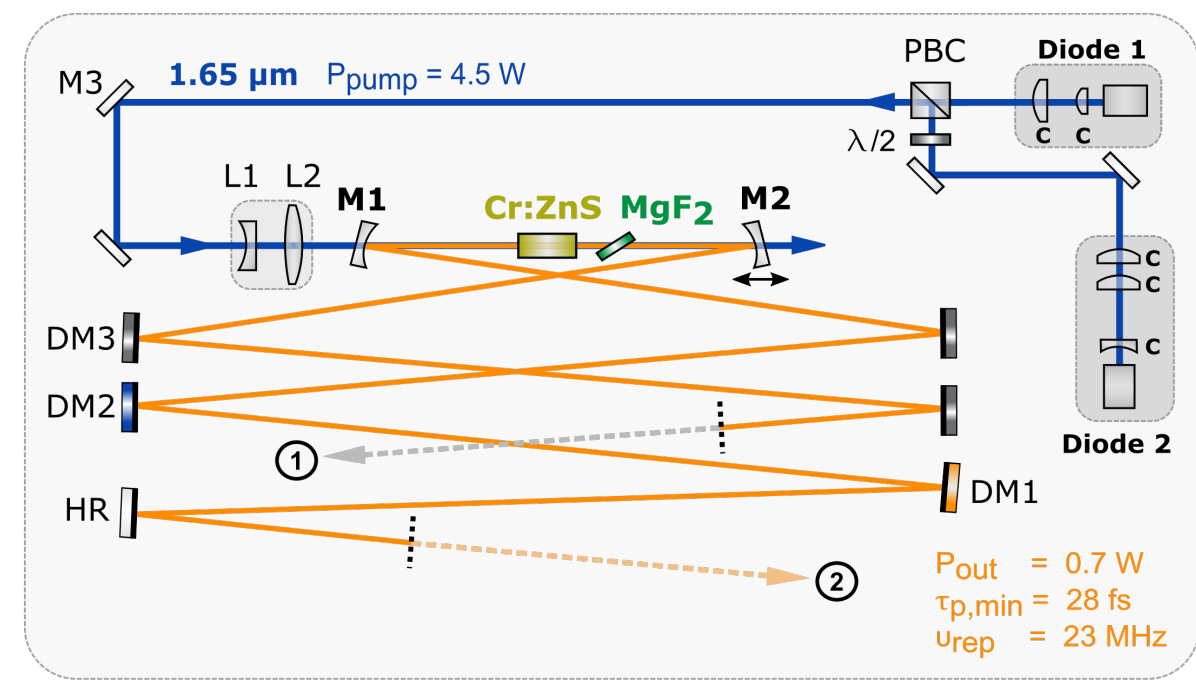

\section{(1) 4f-telescope}

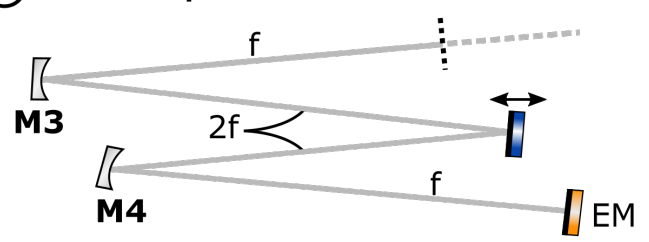

(2) 4f-telescope

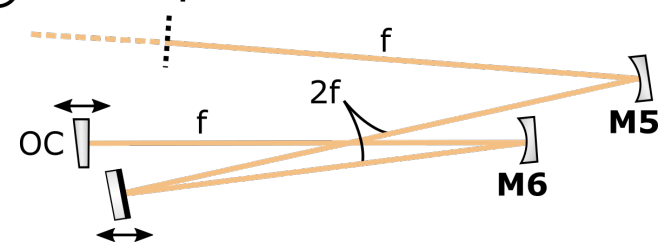

Fig. 5.6: Experimental layout of MIRanda3, including the additional $4 f$-telescope extensions. Diode 1: Type C-132-134, Diode 2: Type C-132-118, C: cylindrical lenses, PBC: polarizing beam combiner, $\lambda / 2$ : half-wave plate, M3: highly-reflective rectangular-shaped mirrors, L1: meniscus lens $(\mathrm{f}=-200 \mathrm{~mm}), \mathrm{L} 2:$ achromatic lens $(\mathrm{f}=80 \mathrm{~mm}), \mathrm{M} 1$ and $\mathrm{M} 2: \mathrm{ROC}=-200 \mathrm{~mm}$, M3-M6: ROC $=-800 \mathrm{~mm}, \mathrm{DM}$ (orange, blue, dark grey): dispersive mirrors, HR (light grey): highly-reflective mirrors, Cr:ZnS: gain medium $(5 \mathrm{~mm})$, Brewster-plate: $\mathrm{MgF}_{2}(3 \mathrm{~mm})$, OC: $25 \%$ output coupler.

A schematic of the experimental setup is depicted in figure 5.6, including two $4 f$-telescopes to extend the cavity arm lengths. However, note that this figure 
represents the oscillator in its final state, and some adjustments to the cavity design were required to achieve optimal laser performance (see subsections 5.2.1.1 and 5.2.1.2). In addition, a new type of C-Mount laser diode was tested (SemiNex Corp., C-132-134), for which the fast axis divergence was matched to the one of the slow axis. Unlike the previous laser diodes, this type required only one cylindrical lens for collimating the fast (f) and slow (s) axis, respectively, making the collimation procedure much more convenient. For diode 1 (C-132-134), cylindrical lenses with $\mathrm{f}=20(\mathrm{f})$ and $\mathrm{f}=75 \mathrm{~mm}(\mathrm{~s})$ were used, whereas a cylindrical lens combination of $\mathrm{f}=-25(\mathrm{f}), \mathrm{f}=100(\mathrm{~s})$ and $\mathrm{f}=150(\mathrm{f})$ was selected to address the output of diode 2 (C-132-118). Moreover, a rail-based assembly was chosen for the pump collimation unit, while the pump focusing lenses were mounted inside a cage system (see pictures 5.7). The gain medium consisted of a $5.1 \mathrm{~mm}$ long AR-coated polycrystalline Cr:ZnS crystal (IPG Photonics) with a clear aperture of $1.8 \mathrm{~mm} \cdot 4.1 \mathrm{~mm}$. Similar to before, a water-cooled copper mount was used as a crystal heat sink and cooled to a temperature of $13^{\circ} \mathrm{C}$, resulting in a measured single-pass absorption of about $79 \%$. The cooling temperature was identical for the pump laser diodes, which were connected to the same external circulating bath unit (termotek, P10040-19192). Compared to the first- and second-generation system, MIRanda3's beam path configuration is much simpler given the classic bow-tie style of the cavity.

(a)

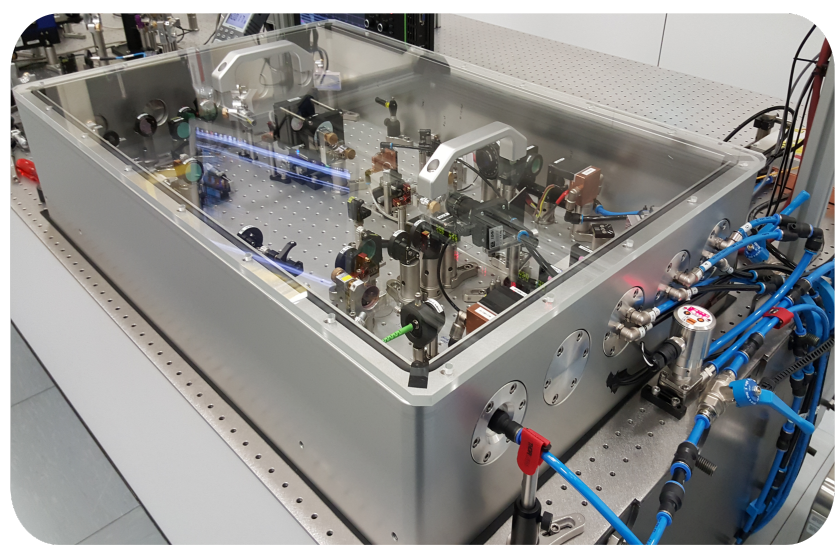

(b)

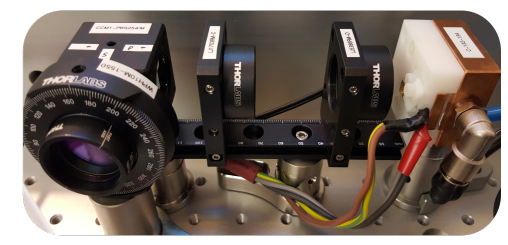

(c)

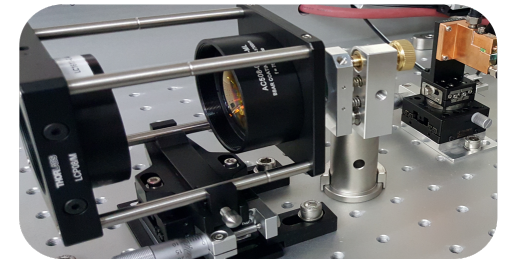

Fig. 5.7: Pictures of the actual laser assembly. (a) Laser housing. (b) Rail-based pump diode setup. (c) Modified pump focusing unit. The latter was based on a cage system and placed on a translation stage for fine-adjusting the pump focus position.

\subsubsection{Intra-Cavity 4f-Telescopes}

To realize an oscillator design with a low repetition rate of about $25 \mathrm{MHz}$, the idea was to build a cavity similar to MIRanda2, but with elongated arm lengths 
of $1900 \mathrm{~mm}$ and $3800 \mathrm{~mm}$, respectively. Also, a combination of $\mathrm{f}=200 \mathrm{~mm}$ and $\mathrm{f}=300 \mathrm{~mm}$ spherical mirrors was chosen such that a similar focal spot size of 85-90 $\mathrm{mm}$ would be obtained in the gain crystal. Experimentally, however, it turned out that the continuous-wave output beam started to significantly degrade for the longer cavity lengths. As shown in figure 5.8, the CW output was measured with the pyroelectric camera and looked clean for cavity lengths corresponding to $50 \mathrm{MHz}$ and $40 \mathrm{MHz}$ repetition rates. However, it started to become larger and slightly less circular for the $25 \mathrm{MHz}$ resonator. In particular, when the output beam was monitored for slightly off-centered positions $( \pm 1 \mathrm{~mm})$ within the cavitie's stability zones, a multi-mode behaviour could be detected. This was attributed to a degradation in mode-matching efficiency: since the pump focal spot diameter $(\sim 95 \mu \mathrm{m})$ and the theoretical cavity mode diameter $(\sim 85 \mu \mathrm{m})$ were already slightly differing at the zone center, an operation closer to the zone boundaries might have enlarged this discrepancy. Thus, the probability of exciting higher-order modes increased.

\section{Stability zone center}

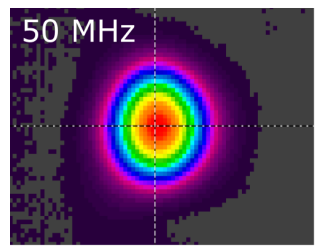

$\varnothing_{\mathrm{f}} \approx 120 \mu \mathrm{m}$

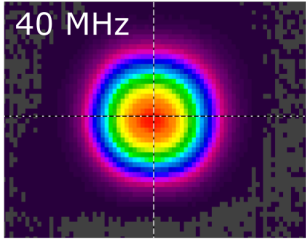

$\varnothing_{\mathrm{f}} \approx 110 \mu \mathrm{m}$

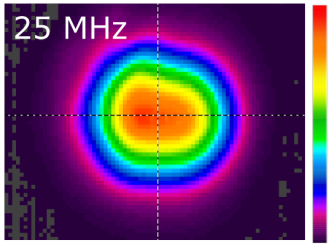

$\varnothing_{\mathrm{f}} \approx 85 \mu \mathrm{m}$
Off-centered

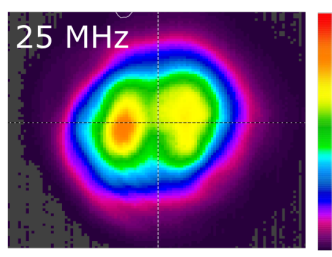

Fig. 5.8: Continuous-wave output beam corresponding to different cavity arm lengths. The theoretical repetition rate is depicted along with the theoretical cavity mode diameter $\varnothing_{f}$ in the focus. The pump spot was as large as $\varnothing_{p} \sim 95 \mu \mathrm{m}$, which explains the degradation in beam quality for the $25 \mathrm{MHz}$ cavity $\left(\varnothing_{p}>\varnothing_{f}\right)$.

Because the pump focus could not be reduced further, a solution was found by extending the cavity arms of a $45 \mathrm{MHz}$ cavity via $4 f$-telescopes (see figure 5.6). Here, the abbreviation " $4 f^{\prime \prime}$ refers to the four separate distances of length $f$ that separate the input plane of a travelling laser beam from its output plane, and a $4 f$ assembly can be thought as an imaging system with unit magnification [281]. Hence, when the ends of both resonator arms are lengthened by $4 f$-telescopes, the beam sizes will effectively not change at the end-mirror positions. Moreover, the beam diameters in the original $45 \mathrm{MHz}$ cavity will stay unaffected - including the focal spot sizes in the gain medium. In the experiment, the resonator was lengthened to a nominal repetition rate of $23 \mathrm{MHz}$ by using spherical mirrors (M3-M6, $\mathrm{f}=-400 \mathrm{~mm}$ ), resulting in a spatially Gaussian single-mode CW operation around the stability zone centers. Next, soft-aperture Kerr-lens mode-locking was tried via a rapid movement and slight misalignment of the output coupler. Since no pulsed operation was achieved, the same was done for the original cavity without the $4 f$-extensions. 
Suprisingly, the oscillator could only be mode-locked for a strong detuning of the OC mirror, resulting in a highly non-single-mode output beam profile as depicted in figure 5.9. The output beam revealed three lobes in the horizontal direction, with the central lobe being the most intense one.

This observation along with the fact that the cavity mode in the crystal is larger than for the first- and second-generation system $(\sim 110 \mu \mathrm{m}$ vs. $\sim 60 \mu \mathrm{m}$ for $M I$ Randa1 and $\sim 90 \mu \mathrm{m}$ MIRanda2) lead to the assumption that the oscillator might favor tighter focal spot sizes for soft-aperture KLM. Moreover, it is worthwhile to mention that the low brightness of the pump laser diodes cannot explain the observed behaviour because the same mode-locked output beam shape was obtained when the oscillator was pumped by a single-mode fiber laser with diffraction-limited beam quality instead (Bktel, HPFL-370-1567-FCAPC). Therefore, the impact of the focal spot size on the oscillator's mode-locking performance was analyzed further.

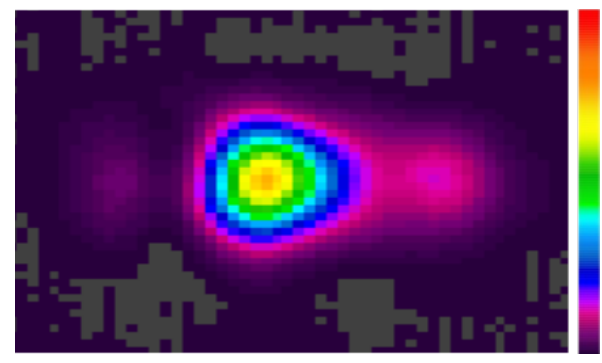

Fig. 5.9: Mode-locked output beam profile for the $45 \mathrm{MHz}$ cavity.

\subsubsection{Selection of the Curved Mirror Pair}

To illustrate the effect of different focal spot diameters on the Kerr strength parameter, two-dimensional simulations were performed on the basis of the nonlinear ABCD-matrix analysis presented in section 4.1.2. For this, the cavity arm lengths were kept constant and the radii of curvature (ROC) of the two spherical mirrors surrounding the $5 \mathrm{~mm}$ long $\mathrm{Cr}: \mathrm{ZnS}$ gain crystal were chosen to be equal. When varying the ROC from $-100 \mathrm{~mm}$ to $-300 \mathrm{~mm}$, the simulated results are shown in figure 5.10 and reveal a clear tendency: because the intensity scale for the Kerr strength parameter was kept the same for all figures, the more intense colouring at the shorter ROC indicates that KLM might be more favorable when tighter spot sizes are present in the gain medium.

As a consequence, the $\mathrm{ROC}=-300 \mathrm{~mm}$ mirror inside MIRanda3 was replaced by a $\mathrm{ROC}=-200 \mathrm{~mm}$ mirror. While this decreases the focal spot diameter from 
$\sim 110 \mu \mathrm{m}$ to $\sim 90 \mu \mathrm{m}$, it can still ensure a good mode-matching with the pump mode $(\sim 95 \mu \mathrm{m})$. Therefore, no further reduction in focus sizes was considered to avoid the onset of multi-mode lasing. Finally, the combination of tighter focusing along with the incorporation of $4 f$-telescopes was successfully implemented in the third-generation system, resulting in a stable single-mode and Kerr-lens mode-locked operation at a low repetition rate of $23 \mathrm{MHz}$.

(a)

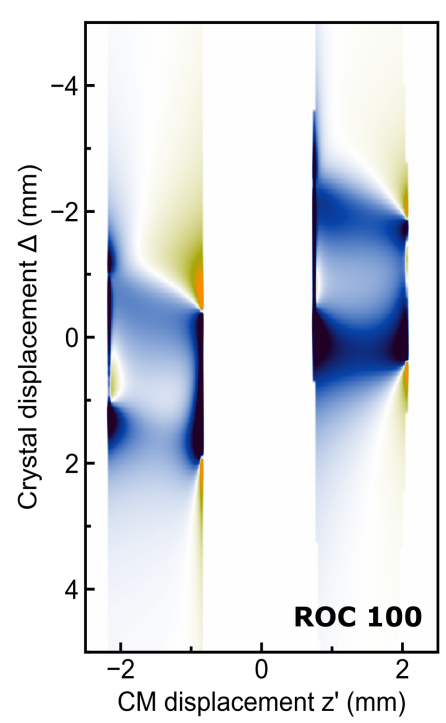

(b)

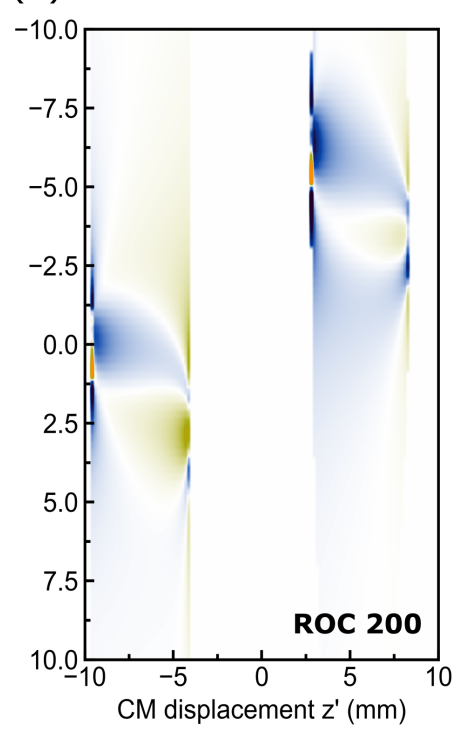

(c)

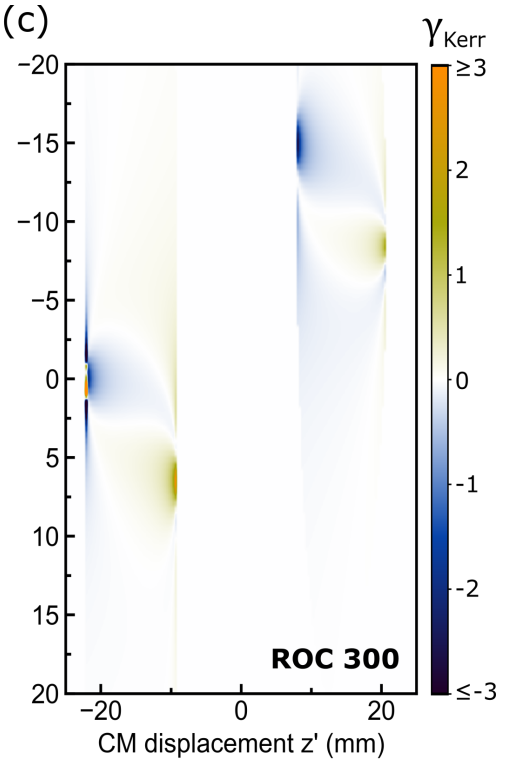

Fig. 5.10: Two-dimensional simulations of the Kerr strength parameter for different radii of curvature (ROC) of the spherical cavity mirrors. The arm lengths were chosen to be $900 \mathrm{~mm}$ and $1870 \mathrm{~mm}$, resulting in a theoretical repetition rate of about $50 \mathrm{MHz}$. For a $5 \mathrm{~mm}$ long Cr:ZnS gain crystal, the results are depicted for (a) $\mathrm{ROC}=-100 \mathrm{~mm}$, (b) $\mathrm{ROC}=-200 \mathrm{~mm}$, and (c) $\mathrm{ROC}=-300 \mathrm{~mm}$.

\subsubsection{Mode-Locked Performance and the Effect of Air Dispersion}

\section{Stability zones}

To locate the oscillator's stability zones and identify parameter ranges that are potentially favorable for soft-aperture Kerr-lens mode-locking, the CW output power of the oscillator was recorded for different mechanical stage positions of mirror M2. Since no Brewster-plate was used in the cavity, the beam astigmatism arising from a reflection on the curved mirrors was uncompensated. Hence, the measured width of the two zones was rather narrow and is plotted in figure 5.11 (a). Therefore, a 
$3 \mathrm{~mm}$ thick $\mathrm{MgF}_{2}$-plate was placed at Brewster's angle in close proximity to the gain crystal (see schematic 5.6). This resulted in enlarged stability zone widths that are in good agreement with the theoretically calculated zones. After this modification, the oscillator layout was not changed anymore, and a linear ABCD-matrix analysis was performed to illustrate the beam radii along the $23 \mathrm{MHz}$ cavity axis (see figure $5.11(\mathrm{~b})$ ). The latter clearly reveals the additional foci created by mirrors M3 to M6, which arise from the extended-cavity structure.
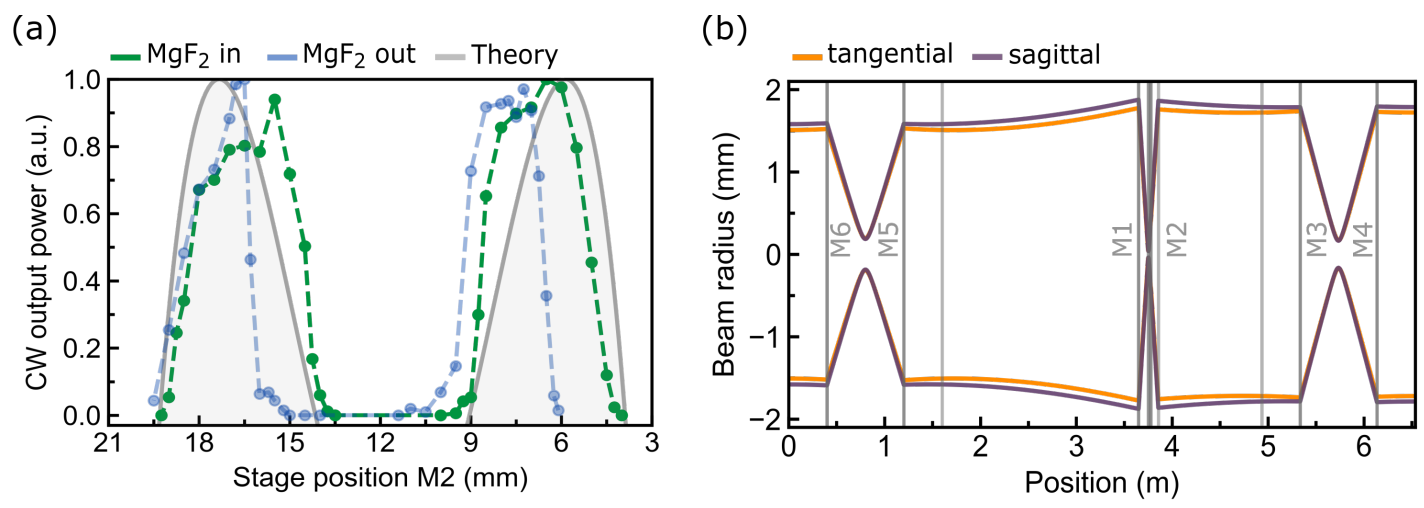

Fig. 5.11: (a) Stability zone scan performed for an output coupling ratio of $1 \%$ and normalized to the maximum CW output power. The theoretical stability zone locations were calculated via linear ABCD-matrix analysis together with (b) the beam radii along the more than 6.5 meter long cavity axis.

\section{Intra-cavity dispersion management}

For the third-generation system, three different dispersive mirror designs (DM1DM3) were used to fine-tune the cavity dispersion. The design of mirror DM1 was the same as for MIRanda2, while the TOD mirror design DM2 has also been used in both MIRanda1 and MIRanda2. To generate even shorter pulse durations, however, the reflection bandwidth of both mirror coatings was slightly improved, which is indicated by the abbreviation "-v5" in figure 5.12. In addition, another dispersive mirror design (DM3) was incorporated into the oscillator, providing a moderate but constant amount of second-order dispersion $\left(\mathrm{GDD} \approx-75 \mathrm{fs}^{2}\right)$ per mirror bounce without adding TOD. After systematically changing the dispersive mirror combinations and recording the corresponding mode-locked output spectra (see illustration in figure 5.13 (a)), it was found that a round-trip combination of 3 bounces on DM1 together with 4 bounces on DM2 and 6 bounces on DM3 was resulting in the shortest output pulse durations for the given set of cavity optics. The corresponding round-trip dispersion is plotted in figure 5.12 (a) and was calculated from the theoretical dispersion values provided by our mirror coating department. Measurements of the mirrors' and output coupler's reflectivity are shown in figure 5.12 (b) and 
reveal a promising highly-reflective behaviour in a broadband spectral range from 1.8-2.9 $\mu \mathrm{m}$. The dips at around $2.7 \mu \mathrm{m}$ are attributed to water absorption and might appear stronger than expected - most likely due to the additional effect of air humidity in the free-space reflectivity measurement setup.

(a)

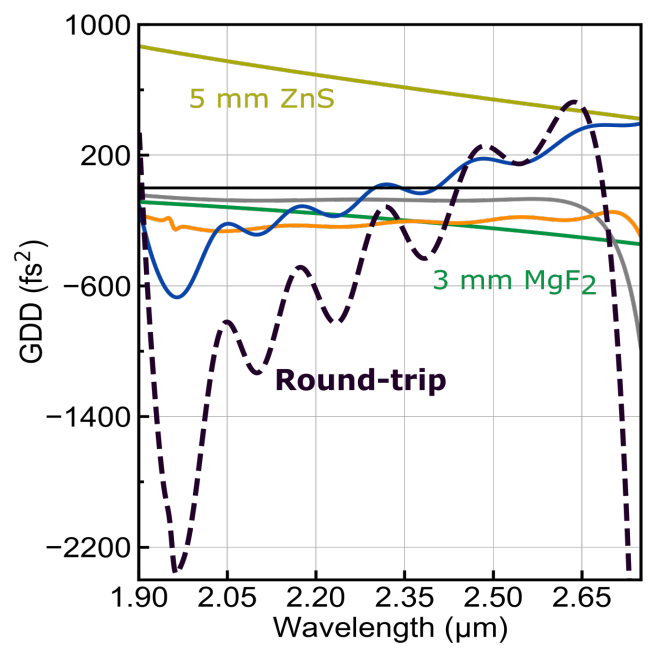

(b)
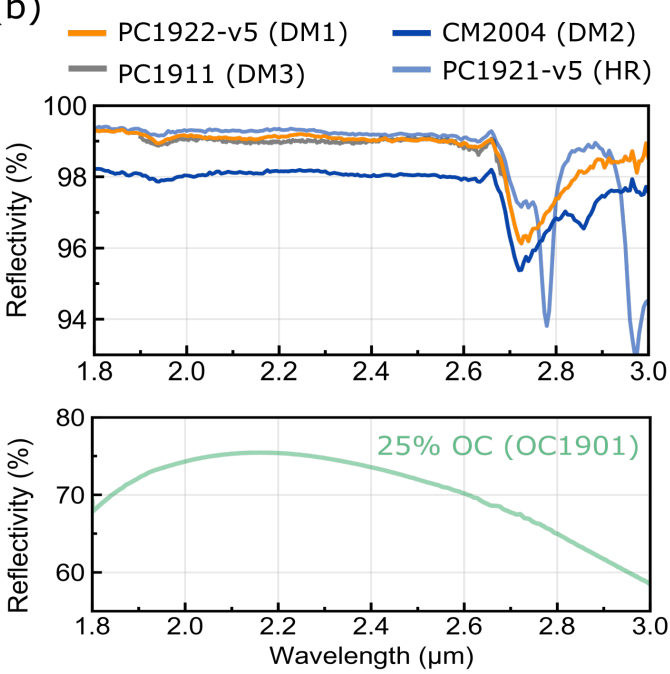

Fig. 5.12: (a) Theoretical dispersion values of intra-cavity elements including the Brewsterplate $\left(\mathrm{MgF}_{2}\right)$ and the gain crystal $(\mathrm{Cr}: \mathrm{ZnS})$ as well as the dispersive mirrors. (b) Measured reflectivity curves (p-polarized) of the highly-reflective (HR) and dispersive (DM1-DM3) mirrors along with the output coupling mirror (OC).

(a)

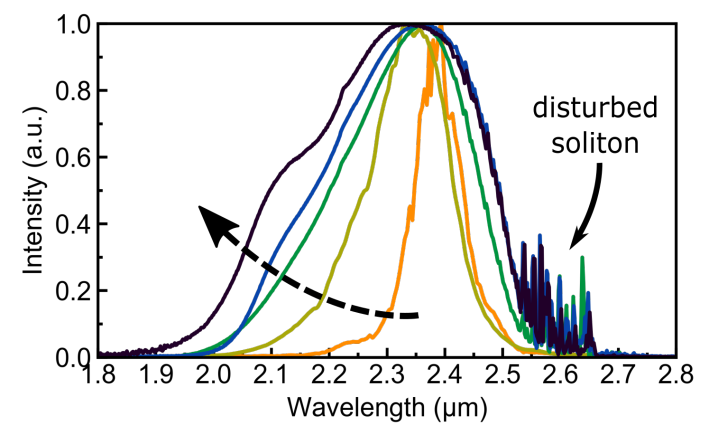

(b)

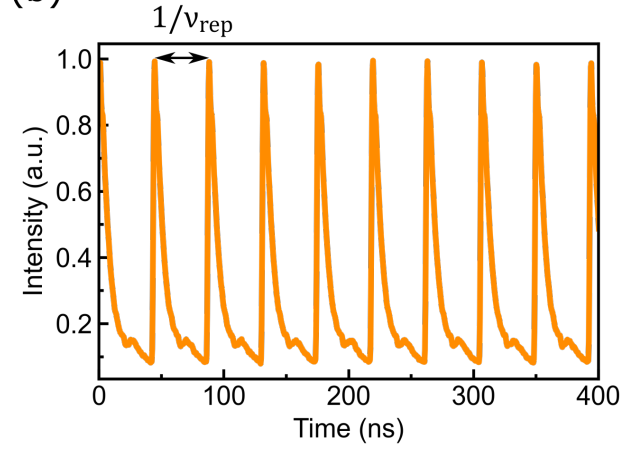

Fig. 5.13: (a) Illustration of the intra-cavity dispersion tuning and the effect on the mode-locked spectral width along with (b) the $\nu_{r e p}=23 \mathrm{MHz}$ output pulse train. The latter was recorded with a fast photodetector (Thorlabs, DET10D2) and oscilloscope (Teledyne LeCroy, HDO4034), while all measured spectra depicted in this chapter were obtained with a grating-based spectrometer ( $A P E$ waveScan 1.5-6.3 $\mu \mathrm{m}$, customized). 


\section{Output power and pulse duration}

At an output coupling ratio of $25 \%$ and a repetition rate as low as $22.9 \mathrm{MHz}$ (see figure $5.13(\mathrm{~b})$ ), the oscillator was delivering average output powers of over $1100 \mathrm{~mW}$ in $\mathrm{CW}$ and $720 \mathrm{~mW}$ in single-mode KLM operation when pumped by the combined emission of the two InP laser diodes (4.5 watts). A further increase in mode-locked power, however, was not possible and resulted in the appearance of pulse instabilites. Note that, unlike the KLM power, the maximum CW value was obtained for an operation at the stability zone center. A SHG-FROG measurement of the output pulses is shown in figure 5.14 with a retrieved FWHM pulse duration of $\tau_{p}=28 \mathrm{fs}$ (FTL: $28 \mathrm{fs}$ ), corresponding to less than four optical cycles. This time, a $100 \mu \mathrm{m}$ thick uncoated lithium iodate crystal $\left(E k s m a, \mathrm{LiIO}_{3}, \theta=20.5^{\circ}\right.$ ) was used for frequency doubling in the FROG device, given that it has intrinsically much more favorable phase-matching properties than the BBO and GaSe crystals which were used so far. According to simulations performed with the open-source software $S N L O$, the SHG acceptance bandwidth of $\mathrm{LiIO}_{3}$ amounts to $112.93 \mathrm{~nm} \cdot \mathrm{cm}$ at $2.3 \mu \mathrm{m}$, whereas it is much lower for BBO $(19.05 \mathrm{~nm} \cdot \mathrm{cm})$ and GaSe $(5.6 \mathrm{~nm} \cdot \mathrm{cm})$. Hence, for a certain crystal thickness, a much broader portion of the pulse spectrum can be phase-matched with a $\mathrm{LiIO}_{3}$ crystal, which offers a high and rather smooth transmission profile for the 2-3 $\mu \mathrm{m}$ polarized light [282].

(a)

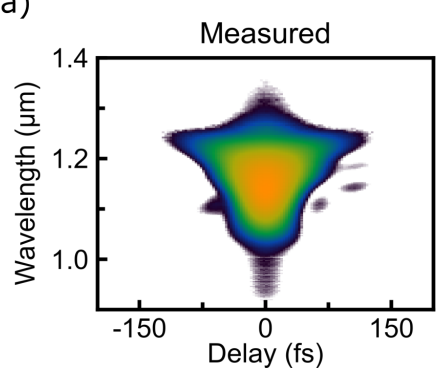

(c)

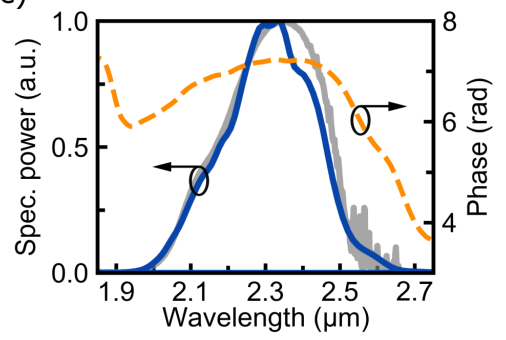

(b)

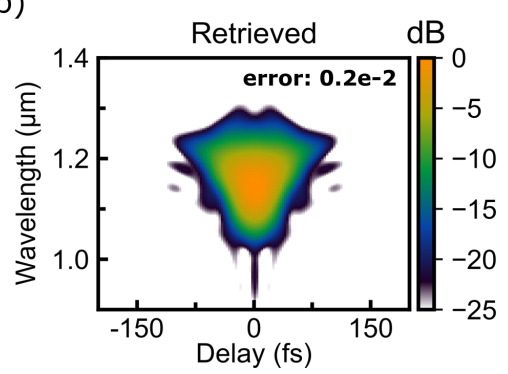

(d)

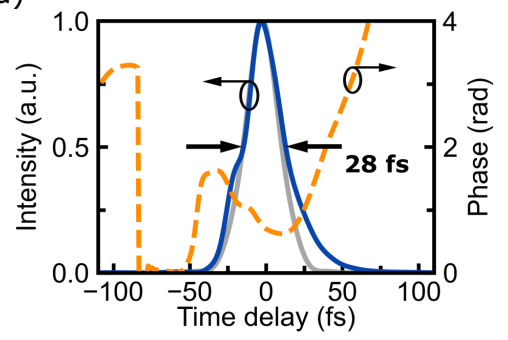

Fig. 5.14: Characterization of the generated laser pulse durations of $M I-$ Randa3 via SHG-FROG measurements. (a)+(b) Measured and retrieved FROG trace. (c) Results in the spectral domain, with the measured spectrum depicted in grey. (d) Results in the temporal domain including the Fourier-transform limited pulse shape (grey). 


\section{The effect of air dispersion}

As explained in the previous section 5.1, the soliton circulating in the laser cavity acquires strong spectral modulations when encountered by some kind of absorbing substance. In the case of $2-3 \mu \mathrm{m} \mathrm{Cr}: \mathrm{ZnS} / \mathrm{ZnSe}$ oscillators, these sharp features arise from water vapor absorption in the 2.6-2.8 $\mu \mathrm{m}$ spectral region and are detectable in the mode-locked output spectra (see figure 5.13 and $[160,278,283]$ ). So far, the performance of all three diode-pumped oscillators presented in this thesis has been optimized under ambient air conditions. To illustrate the effect of air dispersion on the mode-locked laser performance, the laser chamber of this third-generation system was closed and purged with a dry gas. In principle, an evacuation of the system would have also been a viable approach to remove water vapor absorption. However, this has the disadvantage of exerting undesirably high pressure on the $30 \mathrm{~mm}$ thick chamber walls and the $40 \mathrm{~mm}$ thick base, which might cause mechanical deformations at low pressure levels. Purging with a moderate gas flow was thus considered to be more attractive. Typically, dry air or dry nitrogen are used for purging when less humid environments need to be set up. For the results presented in the following, dry nitrogen was preferred over dry air ${ }^{4}$ to reach the lowest possible relative humidity $(\mathrm{RH})$ levels. These RH-values were recorded according to a digital humidity sensor (SENSIRION, SHT30), which is specified for an operation range between $0-100 \%$ RH. Additional efforts were undertaken to seal und purge the remaining path of the laser beam outside the chamber including the pulse characterization diagnostics. However, no significant change was observed in the magnitude of the recorded spectral distortions, and thus the following results were obtained for a purged laser chamber only.

In figure 5.15, the mode-locked output spectra are depicted for three different humidity levels: 41\% RH (starting point), 16\% RH (intermediate level), and 5\% RH (final state). These measurements repeatedly revealed a significant change in the shape of the laser pulse spectrum, which could suddenly be reversed when the gas flow was stopped and the chamber lid was removed - while the laser oscillator was continuously generating ultrashort pulses. The changing behaviour of the spectral shape can be related to a variation in the total dispersion per cavity round-trip, which represents an optical path length as long as 13 meters. Recalling that the diode-pumped laser systems are operated in the regime of dispersion-managed soliton formation (see section 2.3.4), large swings of $\pm D$ between the negative and positive dispersion sections inside the cavity can have a pronounced effect on the solitonic pulse shape. Since the spectra measured at the very low RH-levels down to $5 \%$ became increasingly more flattoped, a higher pulse stretching ratio might have

\footnotetext{
4 "Dry air" — as available in our laboratories — might still contain some rest (though minor) humidity of a few percent.
} 
been present. This ratio is defined as $S=L D / \tau_{F W H M}^{2}$ [65], with the crystal length $L$ and dispersion swing $D$. Hence, to confirm that the pulse duration $\tau_{F W H M}$ of the soliton has decreased and resulted in a larger stretching ratio, a FROG trace was recorded at the lowest relative humidity level of $5 \%$ (see figure 5.15). It resulted in a retrieved pulse duration of $25 \mathrm{fs}$ with a Fourier-transform limit of $23 \mathrm{fs}$.
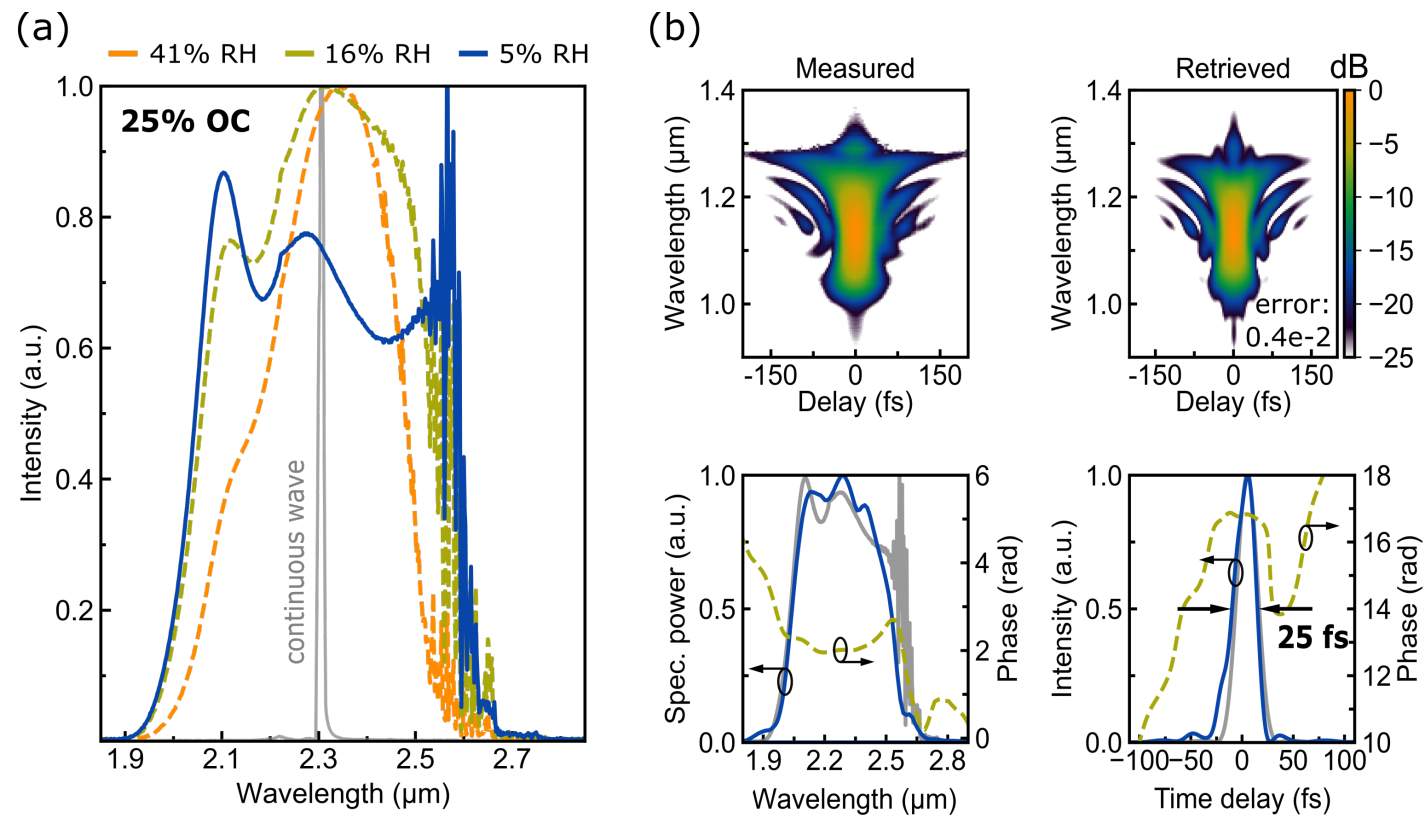

Fig. 5.15: (a) Change of the spectral shape and (b) pulse duration upon purging with dry nitrogen. The FROG trace was recorded for the lowest humidity level of $5 \% \mathrm{RH}$.

However, the Kerr-lens mode-locking performance became notably unstable for a decreasing moisture content, and a reduction in intra-cavity peak power via a decrease in pump power or a shift in gain medium position was necessary to maintain stable pulsed operation. Also, KLM could not be maintained below 5\% RH. This can also be related to the round-trip dispersion converging towards the GDD $=0$ dispersion line, at which soliton mode-locking becomes unstable (recall figure 2.10 from the theory section). Interestingly, strong and sharply pronounced spectral features at the longer wavelengths $(2.5-2.7 \mu \mathrm{m})$ were still present at the very low humidity levels. Hence, even a minor amount of water content in air can significantly perturb the circulating soliton in the resonator.

Since the measured spectral shapes started to resemble the typical output of lasers operating in the regime of chirped-pulse formation (see section 2.3.4) [145-147], a second purging experiment was performed. This time, one of the highlyreflective (HR) cavity mirrors was replaced by a dispersive mirror (DM3) to operate 
the oscillator with slightly added negative dispersion and thus further away from the $\mathrm{GDD}=0$ dispersion boundary. As a result, the oscillator performed better than before when purged, revealing less flattopped spectral shapes and a cleaner SHG-FROG trace (see figure 5.16). Moreover, a low humidity level of $0 \% \mathrm{RH}$ was obtained according to the sensor ${ }^{5}$, with the sharp spectral modulations around 2.6-2.8 $\mathrm{\mu m}$ having almost disappeared. Though a reduction in intra-cavity peak power was again necessary to maintain pulsed operation at low RH-values, a FROG measurement could be performed at the 0\%-level. With a measured temporal FWHM width as low as $22 \mathrm{fs}$ (FTL: $22 \mathrm{fs}$ ), pulses with durations of less than three optical cycles were generated upon purging.

(a)

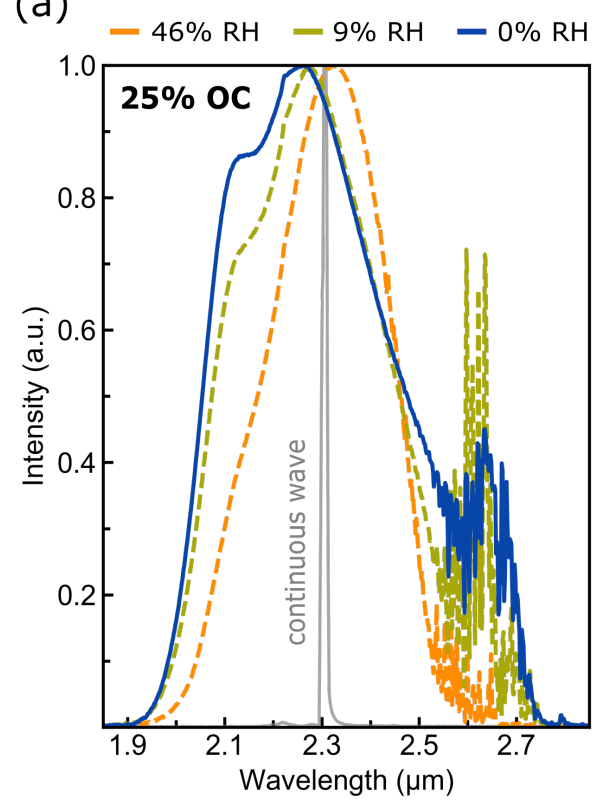

(b)
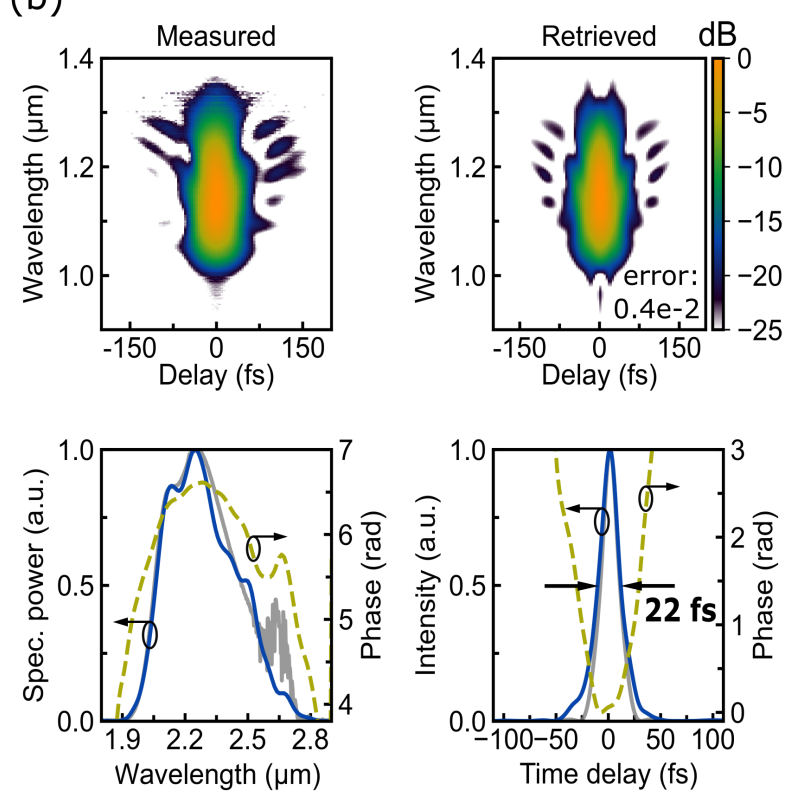

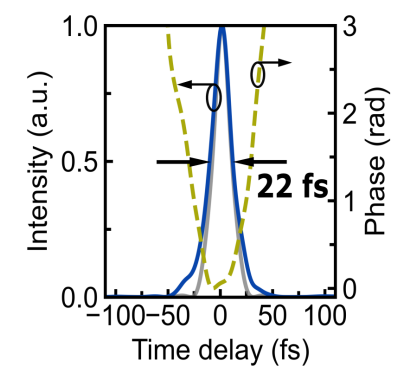

Fig. 5.16: (a) Change of the spectral shape and (b) pulse duration upon purging at slightly increased net negative intra-cavity dispersion. The FROG trace was recorded for the lowest humidity level of $0 \% \mathrm{RH}$. Note that the spectra and the FROG trace appear to be cleaner than those presented in figure 5.15.

\section{Other output characteristics and amplitude noise measurements}

To fully characterize the performance of MIRanda3 when operated at the highest (unpurged) peak power level of nearly $1 \mathrm{MW}$, further measurements were performed and depicted in figure 5.17. With RMS fluctuations of less than $0.10 \%$, the oscillator revealed a stable mode-locked output and single-mode performance over the entire

\footnotetext{
${ }^{5}$ Note that the measurement accuracy of the sensor was specified to be $\pm 4 \% \mathrm{RH}$ at relative humidity levels approaching $0 \% \mathrm{RH}$.
} 
working day ( $>7$ hours). Moreover, the beam quality factors $M^{2}$ for the horizontal (x) and vertical (y) direction were determined to be as low as 1.09 and 1.04, respectively. For recording the slope efficiency curves in CW and KLM operation, the output power of the cavity was measured for different pump power levels similar to the results shown for the second-generation system. With the actual power values of the combined pump beam being measured directly in front of the gain crystal, a continuous-wave slope efficiency of $47 \%$ was obtained, with a value of about $13 \%$ upon mode-locking.

(a)

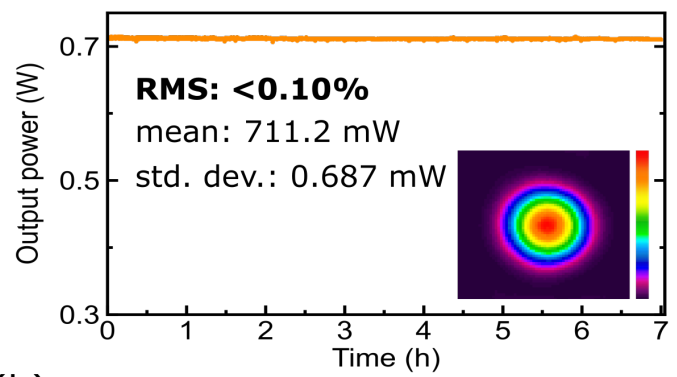

(b)
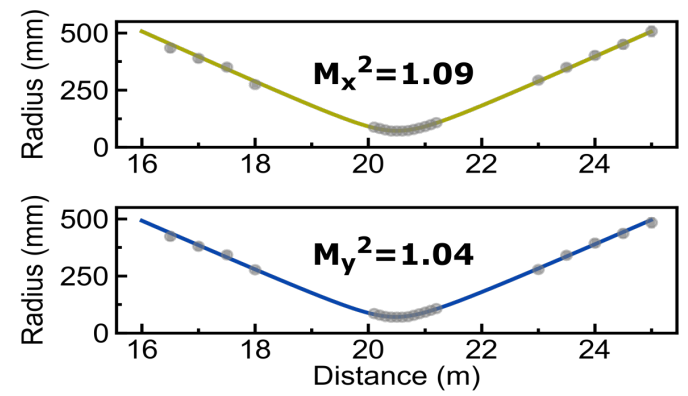

(c)
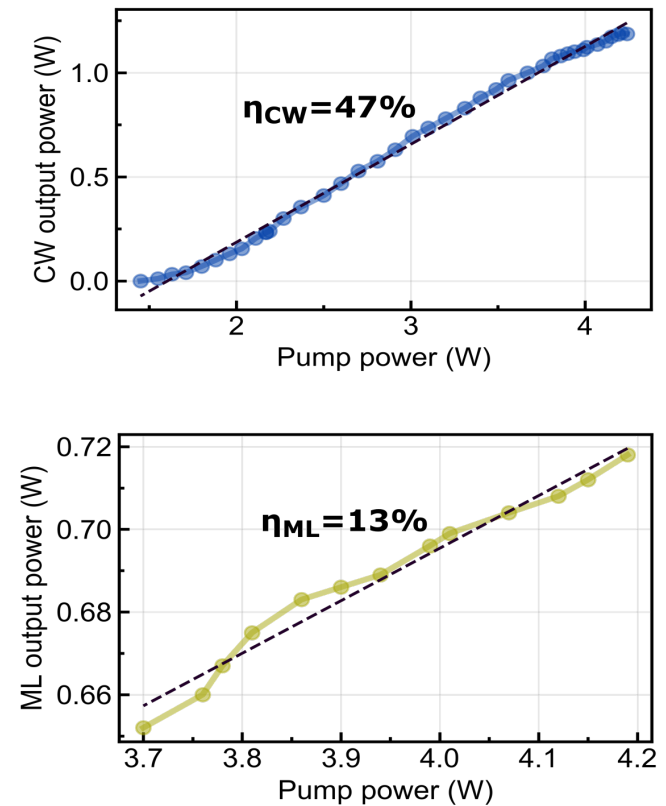

(d)

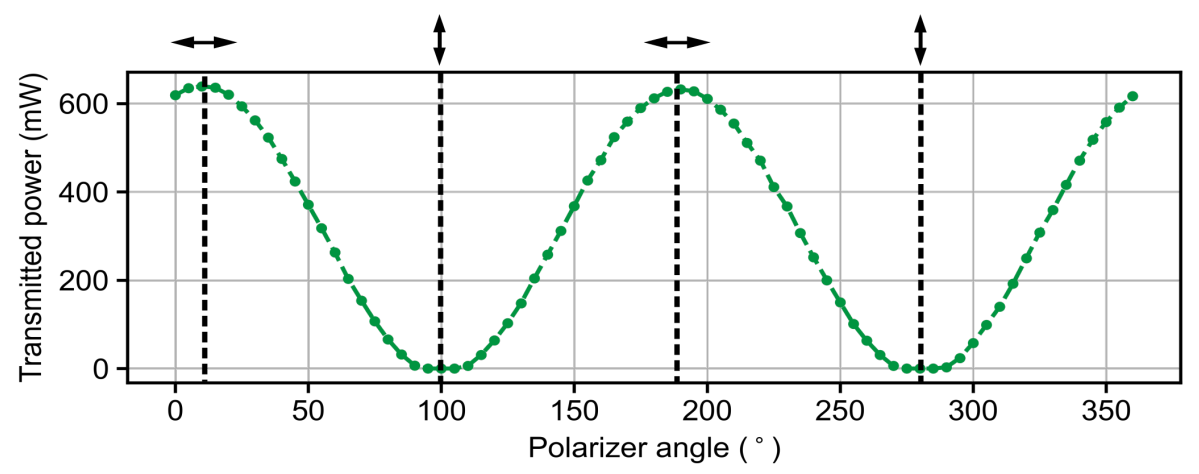

Fig. 5.17: Output characteristics of MIRanda3. (a) Mode-locked output power stability recorded with the thermal power meter (Coherent, PS19) and corresponding single-mode beam profile. (b) $M^{2}$-measurement of the horizontal and vertical beam axis of the mode-locked output. (c) Slope efficiency measured for a decrease in pump current of diode 2 and diode 1. (d) Characterization of the mode-locked output polarization via measuring the transmitted power through a wideband IR polarizer (Innovation Photonics, PGC-5). 
(a)

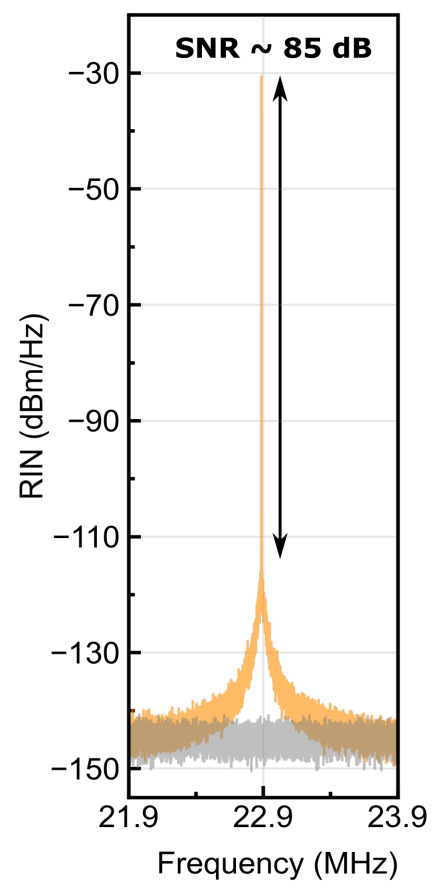

(b)
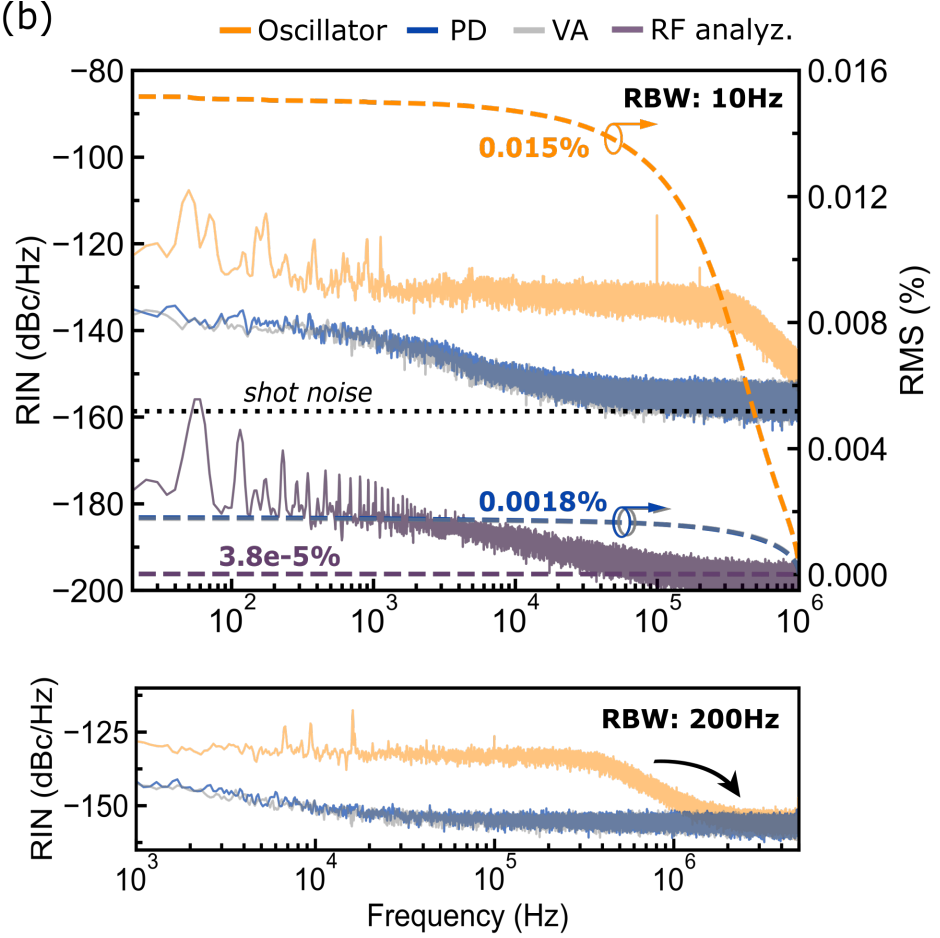

Fig. 5.18: Amplitude noise measurements and integrated RMS noise of the mode-locked output of MIRanda3. (a) RF Spectrum of the carrier region taken without the voltage amplifier. The grey line indicates the noise floor of the detection setup. (b) Amplitude noise measurements in the low-frequency region between $20 \mathrm{~Hz}$ to $1 \mathrm{MHz}$ and $1 \mathrm{kHz}$ to $5 \mathrm{MHz}$, revealing a 'plateau'-like noise without clear noise peaks.

Finally, the polarization of the laser output pulses turned out to be perfectly aligned along the horizontal (p-) direction, with the high polarization extinction ratio being attributed to the additional Brewster plate $\left(\mathrm{MgF}_{2}\right)$ incorporated into the cavity. To demonstrate the low-noise performance of the diode-pumped oscillator - even when running at almost 2.5-times higher intra-cavity peak power levels than before - the same amplitude noise measurement setup was used as presented in the previous chapter (see section 4.2.2). The voltage amplifier (VA) was again operated at the highest gain level of $60 \mathrm{~dB}$, with the theoretically generated DC-voltage ${ }^{6}$ of $U_{D C, 60 d B}=117.7 \mathrm{mV} \cdot 10^{60 / 20}=117.7 \mathrm{~V}$ taken as reference for the $\mathrm{dBc} / \mathrm{Hz}$-conversion. As depicted in figure 5.18, it resulted in integrated RMS noise values of only $0.015 \%$ for the mode-locked ouput between $20 \mathrm{~Hz}$ to $1 \mathrm{MHz}$, which is almost identical to the performance of MIRanda2 (0.014\%). Moreover, the measurement curves of the oscillator noise together with the noise floor of the photodetector (PD) and the voltage amplifier (VA) were finally close to the calculated shot noise level of - $159 \mathrm{dBc} / \mathrm{Hz}$, because higher DC voltage levels could

6 To calculate the DC-voltage $U_{2}$ after amplification with a certain voltage gain factor $G_{V}[\mathrm{~dB}]$, the following formula was used: $U_{2}=U_{1} \cdot 10^{G_{V} / 20}$. 
be obtained without notably saturating the photodiode. Moreover, as expected from the previous measurements, a drop in the 'plateau'-like noise signal to the background noise floor was revealed at frequencies around 1-2 $\mathrm{MHz}$, which arises from the filtering action of the Cr:ZnS gain medium.

\subsubsection{Towards Even Higher Peak Power Levels}

Though peak powers of up to $1 \mathrm{MW}$ were finally realized with directly diode-pumped Cr:ZnS/ZnSe oscillators, the question arises whether further improvements in pulse duration or intra-cavity pulse energies can boost it to even higher levels. Some of the potential methods are briefly addressed in the following:

\section{- Advanced dispersive mirror technology:}

The main problem of using dispersive mirrors for few-cycle laser operation is related to the mirrors' GDD behaviour, which is characterized by unavoidable and irregular oscillations [284]. In particular, when considering the net dispersion per cavity round-trip, theses oscillations might add up dramatically for several bounces on these mirrors (see the theoretical calculations in figures 3.11, 4.12 and 5.12). For a mode-locked laser resonator operating at the vicinity of the $\mathrm{GDD}=0$ dispersion line, a significant effect on the pulse spectral shape can arise, as it was seen from the flattopped and wavy spectra in figure 5.15. To suppress these undesirable oscillations, several approaches exist regarding the design of dispersive mirrors [284]. For the few-cycle pulse durations of $\mathrm{Cr}: \mathrm{ZnS} / \mathrm{ZnSe}$ oscillators and their ultrabroad emission spectrum, the use of dispersive mirror pairs is considered to be a highly promising approach. Introduced about 20 years ago [285, 286], these pairs have identical GDD oscillation amplitudes but the oscillations are shifted by a half period with respect to each other, resulting in very small average GDD oscillations per mirror pair.

Such a design has already been successfully realized for the wavelength range between 400-1200 nm [287], enabling the generation of single-cycle pulse durations from Ti:sapphire laser systems [288, 289]. Just recently, our in-house coating department also managed to fabricate dispersive mirror pairs for the 1.7-3.2 um-operation range of the Cr:ZnS/ZnSe oscillators (see figure 5.19). They are designed to have three mirror pairs being used for compensating the dispersion of the gain crystal $(5 \mathrm{~mm} \mathrm{ZnS})$ and accounting for some additional TOD-slope. The latter could be identified from theoretical round-trip dispersion calculations as presented in this thesis. Though the slope's origin could not be completely clarified and might partially be attributed to air dispersion, its magnitude was consistently of the same strength. Currently, 
first tests are undertaken to assess the quality and performance of the new mirrors as well as to identify potential downsides that need to be improved in future iterations. Once these octave-spanning ${ }^{7}$ mirror pairs proved practial, $\mathrm{Cr}: Z n S / Z n S e$ oscillators with pulse durations approaching the sub-two optical cycle regime $\left(\tau_{p}<15 \mathrm{fs}\right)$ might be feasible.

(a)
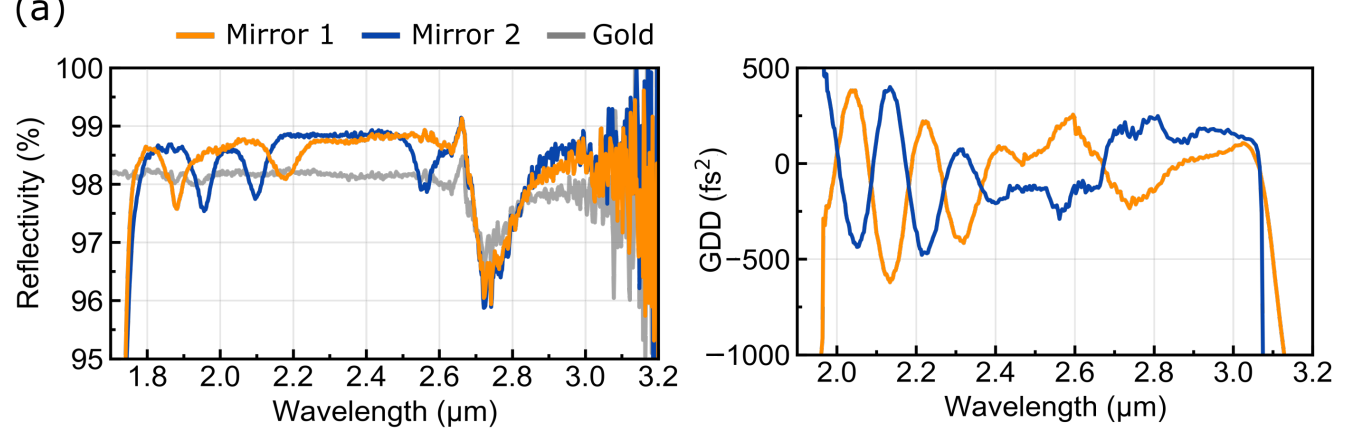

(b)

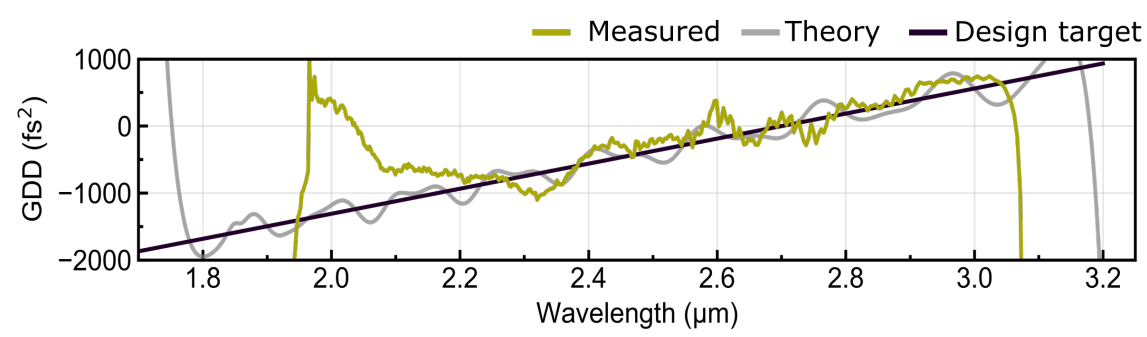

Fig. 5.19: Performance of the newly designed dispersive mirror pair for the 1.8-3.2 $\mu \mathrm{m}$ spectral range. (a) Measured reflectivity and dispersion curves of a pair consisting of mirror 1 and mirror 2. For comparison, the reflectivity curve of a gold-coated sample is shown in grey. (b) Comparison of the total dispersion provided by three dispersive mirror pairs. The black line indicates the original design target. Note that the dispersion measurements started at wavelengths around $2 \mu \mathrm{m}$, and measured GDD-values below $2 \mu \mathrm{m}$ are thus not trustworthy.

\section{- Tighter focal spot diameters:}

As shown from simulations (see figure 5.10), a tighter spot size in the crystal can signficantly increase the Kerr strength (and thus the self-amplitude modulation) and might render the oscillator more robust against the onset of pulse instabilities - especially when heading towards higher peak power levels. Experimental observations obtained with the three diode-pumped laser systems can confirm this behaviour, since no CW-spiking or multi-pulsing could be observed for MIRanda1, given its relatively small focus sizes of only $\sim 60 \mu \mathrm{m}$.

7 For a central wavelength of $2.3 \mu \mathrm{m}$, an optical octave would span the range from about $1.63 \mu \mathrm{m}$ to $3.25 \mu \mathrm{m}$. With a designed operational range of $1.7-3.2 \mu \mathrm{m}$, these mirrors can be considered as "octave-spanning". 
Moreover, reports about powerful fiber-laser-pumped KLM Cr:ZnS/ZnSe oscillators lead to the assumption that their notably higher repetition rates $\left(\nu_{\text {rep }} \geq 75 \mathrm{MHz}\right)$ combined with spherical focusing mirrors of smaller radii of curvature $(\mathrm{ROC} \leq 150 \mathrm{~mm})$ resulted in tighter focal spot diameters of about $\leq 70 \mu \mathrm{m}$ in the gain medium than implemented in MIRanda2 and MIRanda3 $[64,246,290]$. For future diode-pumped cavity designs, it might therefore be advantageous to decrease the focal spot diameters further while keeping the low repetition rate. However, special care must be taken to maintain stable few-cycle KLM operation since the tighter foci automatically call for higher peak intensities and, hence, stronger interplay between nonlinear effects like SAM and SPM in the crystal [144].

\section{- Separate gain and Kerr medium:}

Another potential option could be to completely decouple the gain and the Kerr medium. In traditional KLM-based oscillators, the Kerr-lensing effect and light amplification are both provided by the same laser crystal, imposing certain restrictions on the laser design. As has been demonstrated for KLM thin-disk lasers [184, 291] and diode-pumped KLM bulk lasers [292-294], the use of an additional nonlinear medium inside the cavity can strongly enhance the Kerr-lensing effect, while a complete decoupling between the gain and Kerr medium is also feasible. As a consequence, the gain crystal position can be chosen for maximum laser gain, since the Kerr lensing is optimized independently by choosing suitable positions, thicknesses and materials for the additional Kerr plate. Also, the respective focal spot diameters can be freely adjusted in both the gain and Kerr medium, enabling the use of highly multi-mode fiber-coupled laser diodes $\left(M_{x, y}^{2} \gg 10\right)$ combined with large pump spot diameters in the laser crystal [294]. However, note that the additional telescope increases the range of paramters that need to be adjusted for modelocked operation, and changing the distances within one telescope critically affects the beam diameters in the other telescope [295].

(a)

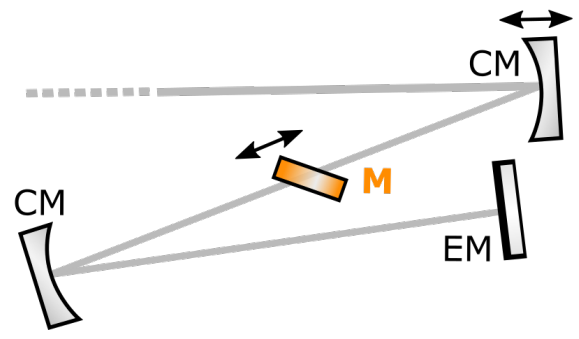

(b)

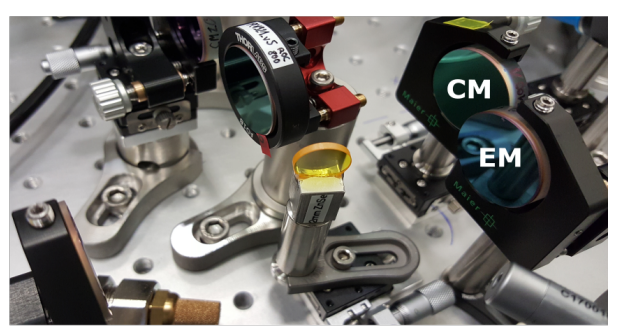

Fig. 5.20: (a) Sketch and (b) picture of the additional telescope added to the laser cavity of MIRanda3 for test purposes. M: $2 \mathrm{~mm} \mathrm{ZnSe}$, CM: curved mirror (ROC $=-100 \mathrm{~mm}$ ), EM: highly-reflective end-mirror. 
A first step into this direction was also performed with the setup of MIRanda3: by replacing the end-mirror (EM) in figure 5.6 with a spherical mirror (CM) (see figure 5.20), the short arm of the cavity was extended by an additional telescope. With short radii of curvature of only $\mathrm{ROC}=-100 \mathrm{~mm}$ and $2 \mathrm{~mm}$ ZnSe chosen as nonlinear medium (M), it was possible to mode-lock the oscillator by varying the position of the ZnSe plate and one of the curved mirrors. Even though ZnSe can provide a slightly larger nonlinear coefficient $n_{2}$ than the ZnS-based gain crystal (see table 2.2), and tighter spot sizes of about 60-70 $\mathrm{mm}$ were present in the additional plate, it was not possible to further improve the laser system's performance. Moreover, an even stronger tendency of the cavity towards multi-pulsing and CW-spiking was observed, most likely due to an increase in the accumulated nonlinear phase shift per resonator round-trip. Hence, instead of decoupling the laser gain and the Kerr effect, the plate might have introduced additional SPM similar to the double-Z cavities demonstrated with octave-spanning Ti:sapphire lasers [61]. Therefore, a reliable test in future generation systems would require a greatly enlarged spot size in the gain crystal to avoid contributions from Kerr lensing.

\section{- Further considerations:}

With the $4 f$-extensions, the repetition rate of the third diode-pumped KLM system was successfully decreased to $23 \mathrm{MHz}$. A further reduction to even lower values might be achieved via cascading of several $4 f$-telescopes or with the aid of a Herriott-style multi-pass cavity [145, 147, 296, 297]. Similar to the $4 f$-imaging method, these multi-pass telescopes are designed to leave the cavity mode invariant when inserted into the laser system, and repetition rates down to $4 \mathrm{MHz}^{8}$ were already demonstrated for KLM Ti:sapphire lasers $[147,298]$.

Furthermore, the use of gain-matched output couplers, whose transmission profile matches the gain spectrum of the laser crystal, was shown to enhance the self-amplitude modulation depth. Moreover, it reduces the need for a strong SAM effect to sustain stable pulsed operation [299, 300]. Also, to scale the peak power of diode-pumped Cr:ZnS/ZnSe lasers to ever higher values, a proper management of the thermal self-focusing in the gain medium will become increasingly important. Hence, new crystal geometries similar to the thin-disk [76] or rod design could be tried for a better heat extraction. Finally, a switch from dispersion-managed soliton formation to chirped-pulse

8 A repetition frequency of $\nu_{r e p}=4 \mathrm{MHz}$ corresponds to an impressively large optical path length of 75 meters per cavity round-trip. With the Herriott-cells, this can be achieved in a rather compact manner. 
formation in the positive dispersion regime (see section 2.3.4) might need to be considered for reaching the highest possible peak power levels with Cr:ZnS/ZnSe lasers [301, 302].

\subsection{Frequency Down-Conversion into the Mid-Infrared}

To demonstrate the power of the third-generation diode-pumped laser system, its mode-locked output was directly sent into a nonlinear crystal (ZGP) for generating broadband mid-infrared via intra-pulse difference frequency generation. Given the high peak power levels of up to $1 \mathrm{MW}$ without external amplification, initial highly promising results were obtained.

\subsubsection{Intra-Pulse Difference Frequency Generation in a ZGP Crystal}

Zinc Germanium Phosphide $\left(\mathrm{ZnGeP}_{2}\right.$, commonly referred to as ZGP) is a positive uniaxial crystal featuring a high laser damage threshold and good thermal conductivity [26]. With its large figure of merit $(\mathrm{FOM})^{9}$ of close to $200 \mathrm{pm}^{2} / \mathrm{V}^{2}$, which is larger than other well-known nonlinear crystals such as GaSe or LGS (see figure 1.3) [26], it is one of the most promising materials for the nonlinear frequency conversion aimed for in this thesis. Because its transparency window only spans from approximately $2 \mu \mathrm{m}$ to $10 \mu \mathrm{m}$ (see figure 5.21 (a)), ZGP needs to be pumped at wavelengths longer than 2 microns. Hence, a series of 2 um-pumped ZGP optical parametric oscillators and amplifiers has already been successfully realized over the past two decades [26, 304-307]. For example, a ZGP OPO was reported to deliver continuously tunable output wavelengths from 3.8-12.4 $\mathrm{mm}$ when pumped at $2.9 \mu \mathrm{m}$ [304], while a combination of ZGP OPO and subsequent ZGP OPA resulted in watt-level average powers driven by a Q-switched Ho:YAG laser $(2.097 \mu \mathrm{m})$ [307].

Just recently, ZGP was identified as a highly attractive nonlinear medium in the context of Cr:ZnS/ZnSe laser-driven intra-pulse DFG. With optical-to-optical frequency conversion efficiencies of up to a few percent $[34,167,308]$, high average powers of over $128 \mathrm{~mW}$ were recorded for the generated MIR wavelengths between 4.5-12.5 $\mathrm{m} \mathrm{[34].} \mathrm{This} \mathrm{power} \mathrm{level} \mathrm{is} \mathrm{much} \mathrm{higher} \mathrm{than} \mathrm{compared} \mathrm{to} \mathrm{similar} \mathrm{experi-}$ ments performed with GaSe [33, 167, 309]. For the IDFG-based setup presented in

\footnotetext{
9 The FOM can be calculated as $d_{\text {eff }}^{2} / n^{3}$, with the crystal's refractive index $n$ and an effective nonlinear coefficient $d_{e f f}$. It is typically used to assess the crystal's potential with regard to nonlinear frequency conversion processes and can be taken as a measure for the expected signal conversion efficiencies [26, 303].
} 
this section, which was directly pumped by the oscillator output, the large conversion efficiencies were considered to be crucial. In the experiment, a ZGP crystal (BAE Systems) with dimensions of $6 \times 7 \times 2 \mathrm{~mm}$ (height $\mathrm{x}$ width $\mathrm{x}$ length) was used, which has been cut for type I phase-matching $\left(\theta=55^{\circ}\right)$. This provides a larger nonlinear coefficient of $d_{e f f}=80.8 \mathrm{pm} / \mathrm{V}$ than type II-based difference frequency generation with $d_{e f f}=74.0 \mathrm{pm} / \mathrm{V}$, allowing for slightly higher nonlinear conversion efficiencies ${ }^{10}$.

(a)

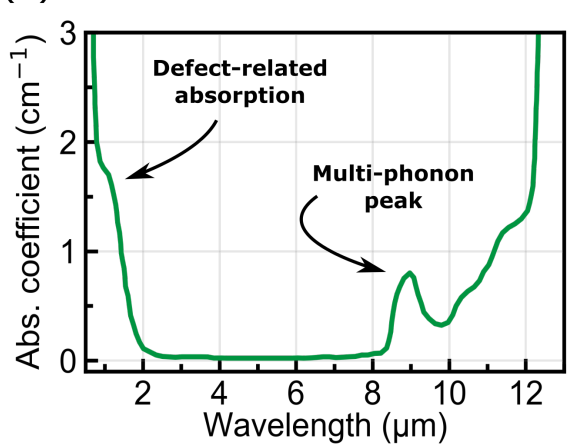

(b)

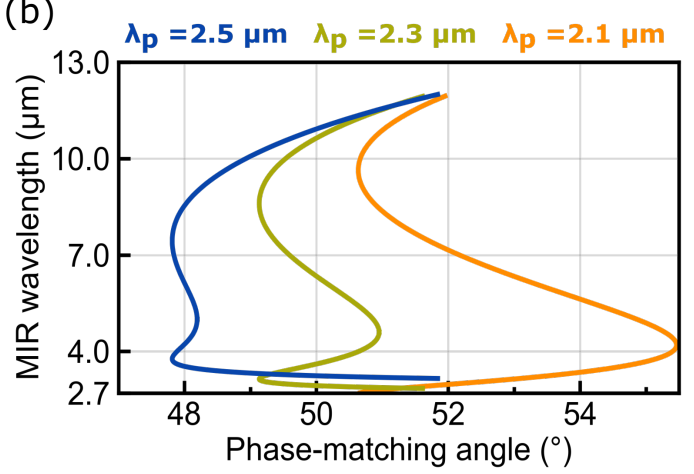

Fig. 5.21: (a) Optical absorption of ZGP (BAE Systems) and (b) simulated type I phase-matching (PM) curves for the generated signal (idler) wavelengths via frequency down-conversion. To mimic the case of intra-pulse DFG, different pump wavelengths were assumed.

To select a reasonable crystal thickness, one can consider the interaction length scale over which DFG takes place. This length is mainly limited by the group velocity mismatch (GVM) between the pump and MIR pulse [167], which causes the two pulse envelopes to separate from each other after a certain distance $L_{\text {eff }}$. Assuming a central pump wavelength of $2.4 \mathrm{\mu m}$ and mid-infrared wavelength of $10 \mu \mathrm{m}$, it can be calculated with the group velocities obtained from $S N L O$ :

$$
L_{e f f}=\frac{\tau_{p}}{G V M}=\frac{\tau_{p}}{\left(v_{g, 2.4 \mu \mathrm{m}}\right)^{-1}-\left(v_{g, 10 \mu \mathrm{m}}\right)^{-1}}=\frac{28 \mathrm{fs}}{3.22928 / c-3.21953 / c} \approx 861 \mathrm{\mu m}
$$

with $c$ denoting the speed of light. Though only a $2 \mathrm{~mm}$ thick ZGP crystal was available, even thinner substrates might be considered for future experiments. Also, the crystal was provided with an AR-coating for the wavelengths between 2.04-2.2 $\mathrm{\mu m}$ and 2.8-6 $\mathrm{mm}$, which was optimized for another experiment and not for the IDFG with the broadband output of the Cr:ZnS oscillator. Nonetheless, first measurements could be successfully performed and can serve as a useful reference for future experiments.

10 The $d_{e f f}$-values were obtained from simulations with the software $S N L O$, assuming a pump and signal frequency of $2.0 \mu \mathrm{m}$ and $2.6 \mu \mathrm{m}$, respectively. 


\subsubsection{Characterization of the Generated Radiation}

The ZGP crystal was placed into the focus of a parabolic mirror (see figure 5.22 (a)) with a corresponding focal spot diameter of about 35 microns measured with a rotating-slit beam profiler (Ophir Optronics Inc., NanoScan 2s). The transmitted beam was re-collimated by another parabolic mirror of the same focal length. In addition, the crystal was mounted on top of a mechanical translation stage and placed inside a rotational mount to move it along the pump focusing direction and to satisfy the phase matching condition ${ }^{11}$. Thus, by adjusting the orientation of the crystal, the polarization vector of the incident pump E-field was aligned to have approximately equal $e$ and $o$ components with respect to the crystal axis.

As a first step, the mid-infrared power levels $(>4.5 \mu \mathrm{m})$ as well as the total transmitted power behind the ZGP crystal were analyzed at different crystal positions along the propagation axis (Z-axis). The result is depicted in figure 5.22 (b) together with the corresponding beam profiles that were recorded with a pyroelectric camera (Ophir Optronics, Pyrocam III). Here, three different cases are distinguished: (i) the continuous-wave (CW) and (ii) mode-locked (ML) beam profiles measured at the fundamental wavelengths, and (iii) the mode-locked beam profiles that were analyzed after a $4.5 \mu \mathrm{m}$ long-pass filter. In the first case, no degradation in beam quality was observed when the crystal was moved in and out of focus, and a single-mode beam profile was detected for various different Z-axis positions. In the second case, however, a spatially Gaussian beam intensity distribution could only be recorded when the crystal was moved at least $\pm 1 \mathrm{~mm}$ away from the focus $(\mathrm{Z} \approx 5.3 \mathrm{~mm})$. As soon as the nonlinear medium entered the focal area at Z-values between $4.3-6.2 \mathrm{~mm}$, the beam profile significantly degraded, but it recovered when the ZGP crystal was placed outside the focusing region again. Also, a similarly strong distortion was observed for the generated mid-IR radiation at wavelengths greater than $4.5 \mathrm{\mu m}$. In addition to the beam profile measurements, the total transmitted power was analyzed immediately behind the crystal and resulted in a pronounced drop around Z-positions between 4.50-6.00 mm. Surprisingly, no crystal damage was observed due to the recovery of the absorption and the measured transmitted powers when moving the crystal in and out of focus. Given this strong absorption in the fundamental power, however, the appearance of mid-infared radiation was shifted to regions of reduced peak intensities, and a maximum in the generated MIR power was measured on both sides of the dip (see figure 5.22 (b)).

11 For the DFG process, the type I phase-matching in a positive birefringent crystal can be written as: $\lambda_{p}(\mathrm{o}), \lambda_{s}(\mathrm{e}) \rightarrow \lambda_{i}(\mathrm{e})$, with $e$ and $o$ denoting the extraordinary and ordinary polarization of the pump (p), signal (s) and idler (i) beams, respectively. $\lambda_{i} \geq \lambda_{s}>\lambda_{p}$ [67]. 
(a)

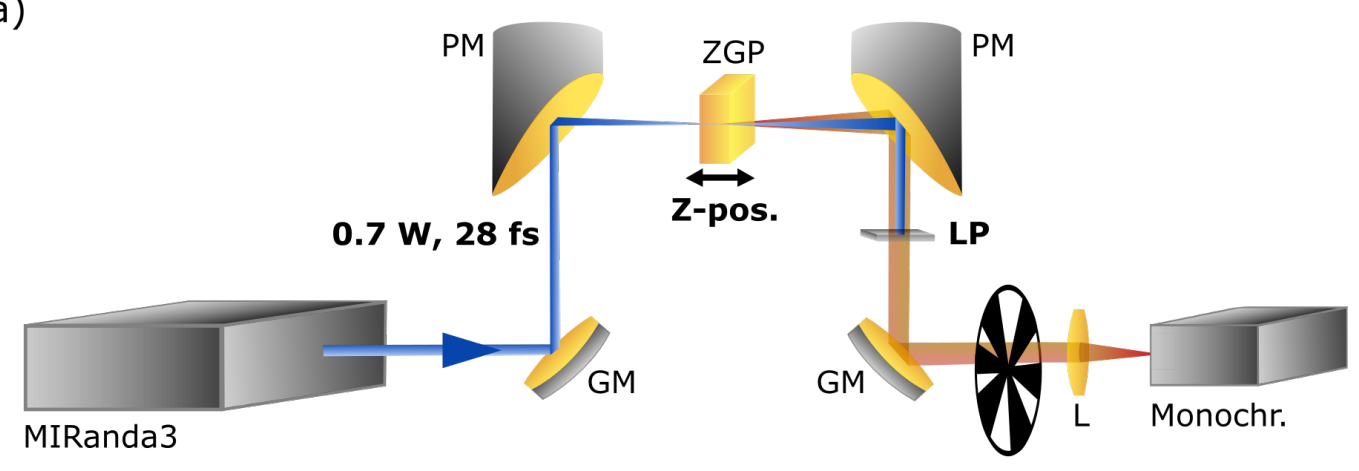

(b)

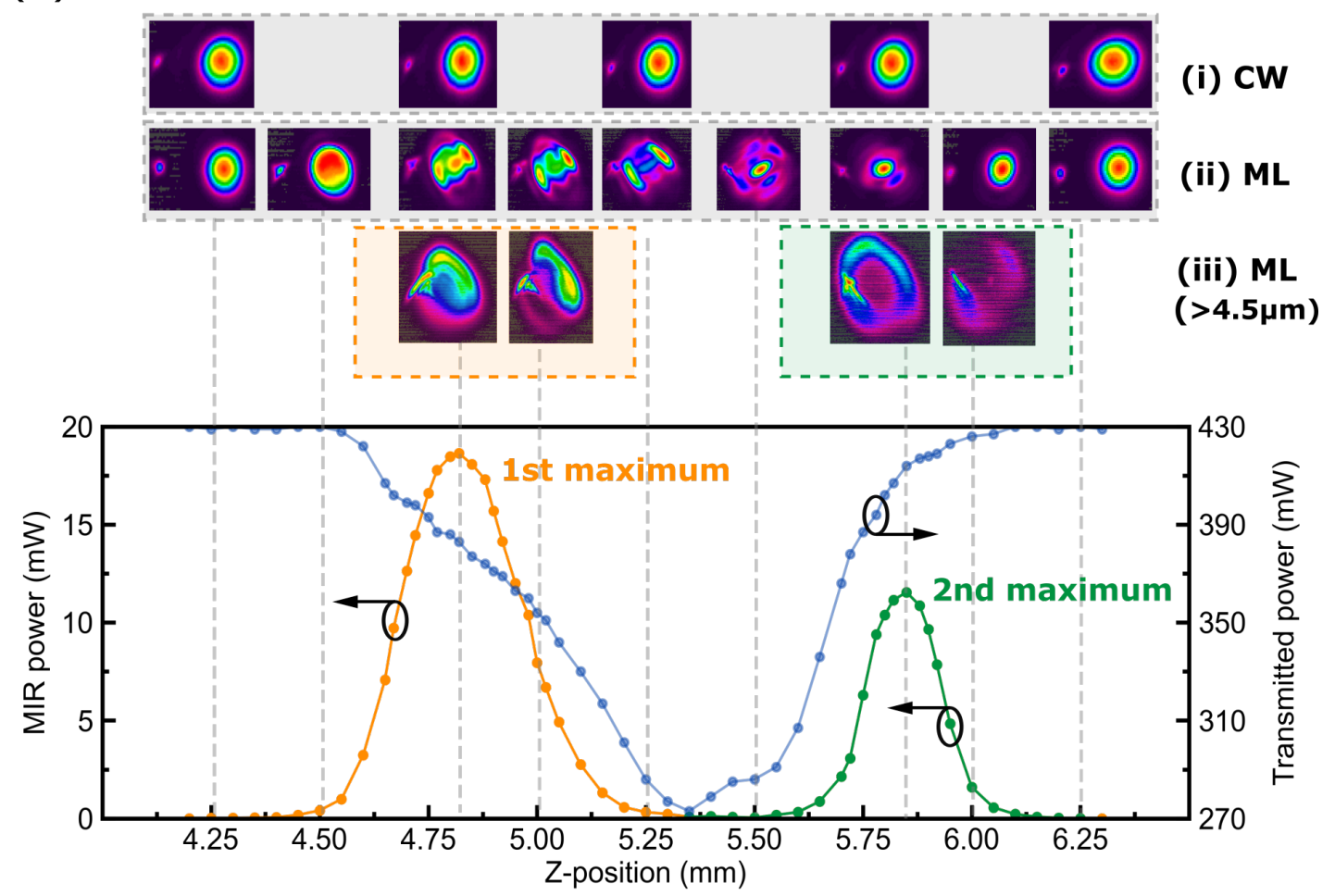

Fig. 5.22: Mid-infrared generation setup along with the measured power levels and beam profiles behind the ZGP crystal. (a) Setup, including two gold-coated $90^{\circ}$ off-axis parabolic mirrors (PM, $\mathrm{f}=25.4 \mathrm{~mm}$ ), gold-coated mirrors $(\mathrm{GM})$, a long-pass filter (LP), a monochromator, a ZGP crystal $(\mathrm{d}=2 \mathrm{~mm})$, and a ZnSe lens $(\mathrm{L}, \mathrm{f}=75 \mathrm{~mm})$. (b) Generated mid-infrared power $(>4.5 \mu \mathrm{m})$ and transmitted total power behind the $2 \mathrm{~mm}$ thick ZGP crystal, revealing two maxima (orange/green). Increasing Z-values denote a movement of the crystal towards the second parabolic mirror. Also, the corresponding beam profiles were recorded at the fundamental wavelengths for (i) continuous-wave (CW) and (ii) mode-locked (ML) operation as well as for (iii) the MIR radiation behind a $4.5 \mu \mathrm{m}$ long-pass filter $(\mathrm{ML}>4.5 \mu \mathrm{m})$. Interestingly, the shape of the distorted beam profiles reveals some kind of symmetry, similar to the cylindrical transverse mode patterns (TEM) presented in the theoretical section (figure 2.1). 
For the fundamental wavelengths, the pronounced degradation of the spatial beam profiles and the reduction in transmitted power is most likely due to the interplay between strong multi-photon absorption and self-focusing [310]. According to table 2.2, ZGP features a high refractive index of $n_{0}=3.14$ and a comparably large nonlinear coefficient of $n_{2} \approx 550 \cdot 10^{-16} \mathrm{~cm}^{2} / \mathrm{W}$, and it has a bandgap energy of $2 \mathrm{eV}$ [311]. Hence, its critical power for self-focusing amounts to only $P_{c r}=45.4 \mathrm{~kW}$ (see definition in section 2.1.2), which is much lower than the mode-locked peak power $P_{0} \approx 1 \mathrm{MW}$ delivered by MIRanda3. Indeed, it was observed that the collimating parabolic mirror needs to be adjusted for different crystal position for proper collimation. When the crystal is placed at the focus, where the spot diameter is $35 \mathrm{\mu m}$, the beam should have theoretically collapsed to an arbitrarily small size after propagation through $z_{f} \approx 573 \mu \mathrm{m}$ of the $2 \mathrm{~mm}$ thick ZGP crystal. This propagation distance $z_{f}$ was calculated according to $z_{f}=k \cdot a_{0}^{2} \cdot\left(P_{0} / P_{c r}-1\right)^{-0.5}[312]$, including the spot radius $a_{0}$ and the wave vector $k=2 \pi \cdot n_{0} / \lambda_{0}$ at a central wavelength of $\lambda_{0}=2300 \mathrm{~nm}$. However, the increasing mulit-photon absorption at high intensities might have arrested the collapse of the laser beam, and therefore no permanent damage of the ZGP crystal was observed.

The interaction between self-focusing and multi-photon absorption is highly sensitive to the initial intensity distribution of the incident beam. As multi-photon absorption scales with the laser peak intensity, the resulting beam profile will not be radially symmetric if the initial beam is slightly elliptical, as in our case. Furthermore, compared to the edges, the central portion of the original fundamental beam $\left(\mathrm{TEM}_{00}\right)$ will experience higher absorption rate and thus lower intensities after propagation through the crystal. These effects could also explain the ring or donut shaped beam profiles of the generated MIR light, which correlate with the intensity distribution of the fundamental beam within the crystal (see figure 5.22 (b), case (iii)). Note that laser beam filamentation associated with plasma formation is unlikely to play a role here, given the nanojoule-level of pulse energies used in this experiment $[81,313]$. However, it is remarkable that a degradation in beam quality has not been reported in previous experiments [34, 167, 308]. Therefore, further measurements are planned in the future to better understand the physical origin behind the observed effects.

To analyze the generated MIR spectra, a Czerny-Turner monochromator (Newport, Cornerstone 260) was combined with a lock-in amplifier (Stanford Research Systems, SR810) and a mechanical chopper to modulate the intensity of the laser beam. For detection, a lithium tantalate pyroelectric detector (Oriel Instruments, DET-L-PYK5-R-P) was attached to the monochromator and connected with the lock-in amplifier, allowing for highly sensitive detection of the weak mid-infrared radiation. This measurement system allowed to detect wavelengths between $0.725 \mu \mathrm{m}$ to $20 \mu \mathrm{m}$ since the monochromator was equipped with three gratings to cover different 
spectral portions: $0.725-3.25 \mu \mathrm{m}$ (grating 1), 2.5-11 $\mu \mathrm{m}$ (grating 2), and 4.5-20 $\mu \mathrm{m}$ (grating 3). In addition, suitable long-pass filters with cut-on wavelengths at $2.8 \mu \mathrm{m}$, $4.5 \mu \mathrm{m}, 7.3 \mu \mathrm{m}$, and $11 \mu \mathrm{m}$ were used to suppress second-order diffractions arising from the monochromator's gratings and to analyze the signals between 2.5-5.6 $\mu \mathrm{m}$,

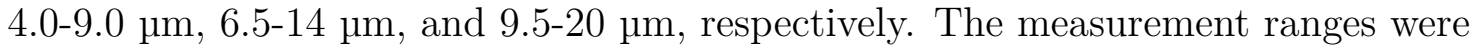
chosen slightly larger to provide sufficient data points for the subsequent stitching process.

(a)

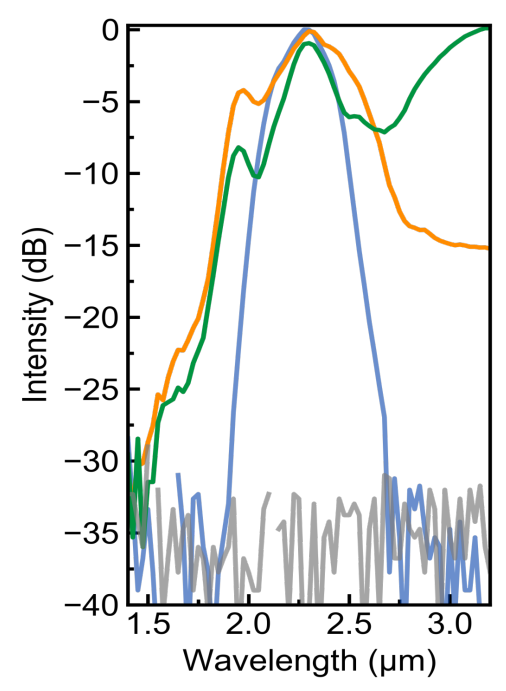

(b)

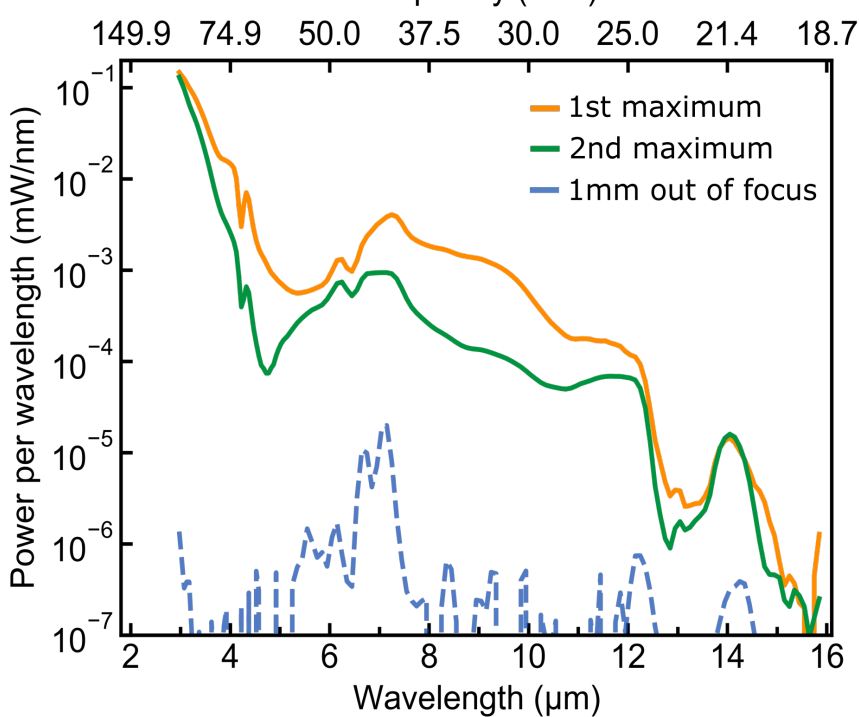

Fig. 5.23: Generated (mid-)infrared spectra with a ZGP crystal for the two observed maxima. (a) Data recorded for the fundamental wavelength region of the laser oscillator, indicating the presence of SPM-induced spectral broadening. The blue curve resembles the mode-locked spectrum of the oscillator, and the grey line denotes the detection noise floor. (b) Generated mid-infrared wavelengths via cascaded parametric processes. The measured spectra were calibrated and stitched together to be displayed at normalized units of $(\mathrm{mW} / \mathrm{nm})$.

The intensities of the measured MIR spectra beyond $2.8 \mu \mathrm{m}$ were calibrated with the aid of a silicon nitride infrared emitter (Bentham Instruments Ltd., CL2-IR) to account for the wavelength-dependent efficiency variation of the monochromator gratings and the filter losses. Also, the transmission curve of the ZnSe lens placed in front of the detection system was taken into account. When stitching the intensity-corrected measurements together and normalizing the intensity axis to the respective power levels measured behind the long-pass filters, a final spectrum was obtained (figure $5.23(\mathrm{~b})$ ). The average powers were recorded with a thermal power meter (Coherent, PS19) and are summarized in table 5.3. Note that the real values are most likely even higher, given that the transmission curve of the filters varied strongly between $70-90 \%$ for the measured MIR wavelengths. 
The generated MIR spectrum covers about two optical octaves in the wavelength range from 2.8-14 $\mathrm{m}\left(714-3571 \mathrm{~cm}^{-1}\right)$, and thus a great variety of molecular absorption lines in the gas and liquid phase could already be addressed (see figure 1.1). Interestingly, the spectral components below $7.3 \mu \mathrm{m}$ cannot be explained by simple intra-pulse DFG alone, given that the mode-locked oscillator spectrum has spectral limits of $\lambda_{1}=1.95 \mu \mathrm{m}$ and $\lambda_{2}=2.66 \mu \mathrm{m}$ at the $-20 \mathrm{~dB}$ level ${ }^{12}$. In fact, the recorded spectrum reveals a peak near $7.3 \mu \mathrm{m}$, with a substantial amount of signal being located at even shorter mid-infrared wavelengths. As explained and simulated in references [33, 243], a cascading effect takes place here: the generated fundamental DFG components are further mixed with the initial driving laser frequencies, and several cascading processes set in to generate an even broader range of spectral components. The corresponding background level in figure 5.23 (b) was obtained by shifting the nonlinear crystal $1 \mathrm{~mm}$ away from the focus to suppress any nonlinear effects. Besides the nonlinear mixing, also spectral broadening of the oscillator input spectrum down to $1.5 \mu \mathrm{m}$ was observed (see figure 5.23), being attributed to self-phase modulation at the fundamental wavelengths. When taking into account the average transmission of about $85 \%$ for the $4.5 \mu \mathrm{m}$ long-pass filter, the optical-to-optical conversion efficiencies amounted to $5.7 \%$. This demonstrates the great potential of using powerful diode-pumped $\mathrm{Cr}: \mathrm{ZnS} / \mathrm{ZnSe}$ oscillators for direct MIR generation.

\begin{tabular}{lcc} 
Filter & 1st maximum & 2nd maximum \\
\hline $2.8 \mu \mathrm{m}(2 \mathrm{x})$ & $75 \mathrm{~mW}$ & $42 \mathrm{~mW}$ \\
$4.5 \mu \mathrm{m}$ & $20.7 \mathrm{~mW}$ & $11.9 \mathrm{~mW}$ \\
$7.3 \mu \mathrm{m}$ & $15.8 \mathrm{~mW}$ & $7.5 \mathrm{~mW}$ \\
$11 \mu \mathrm{m}$ & $1.3 \mathrm{~mW}$ & $1.0 \mathrm{~mW}$
\end{tabular}

Table 5.3: Average power levels measured directly behind different long-pass filters for the 1st and 2nd maximum.

With regard to future experiments, the setup can be further improved to exploit the full potential of the IDFG-based system. For example, when analyzing the corresponding B-integral in the ZGP crystal for the measured focal spot diameter of $35 \mu \mathrm{m}$, a very large value of close to $\mathrm{B}=15$ is calculated (see figure 5.24 ), which may also contribute to the strong degradation of the spatial beam profile. By contrast, a larger focus size of $120 \mu \mathrm{m}$ would significantly reduce the B-integral to below 2.5. In addition, it improves the beam overlap between the fundamental and the generated mid-infrared beam given the decreased beam divergences. A thinner crystal with

$\overline{12}$ Recall that the wavelengths generated via DFG are calculated through $\lambda_{3}^{-1}=\lambda_{1}^{-1}-\lambda_{2}^{-1}$, which amounts to $\lambda_{3}=7.31 \mathrm{\mu m}$ for the considered minimum and maximum wavelengths. 
a slightly optimized phase-matching angle of $\theta \approx 50^{\circ}$ might also be selected (see simulations presented in figure 5.21 (b)) along with a more broadband AR-coating to push the conversion efficiencies to ever higher values.

(a)

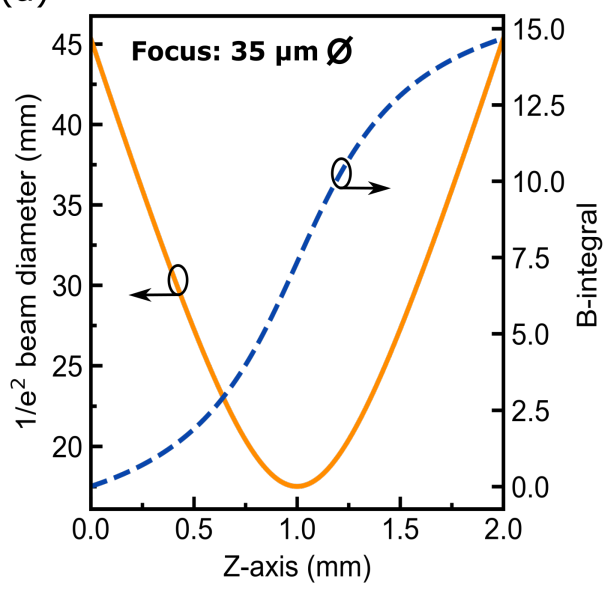

(b)

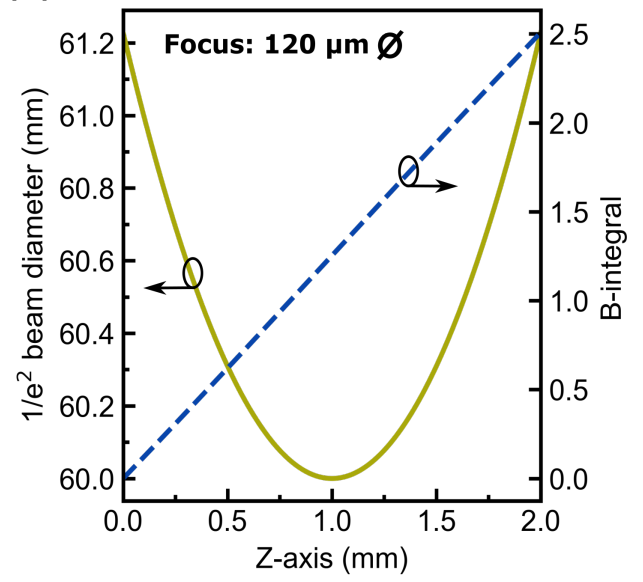

Fig. 5.24: Calculation of the single-pass B-integral inside the ZGP crystal $\left(n_{2}=5.5 \cdot 10^{-18} \mathrm{~m}^{2} / \mathrm{W}\right)$. The smaller the focal spot diameter, the higher the accumulated nonlinear phase shift (B-integral).

\subsection{Chapter Summary}

With this third-generation system, the performance of directly diode-pumped $\mathrm{Cr}^{2+}$ doped II-VI lasers has been brought to a new level. With the aid of a novel pump focusing approach and a classic bow-tie design of the cavity, a powerful oscillator with peak powers of up to $1 \mathrm{MW}$ has been realized. This constitutes an almost 2.5-fold increase in peak power over the previous diode-pumped generation, which was pumped in a similar fashion by the combined emission of two single-emitter laser diodes. Moreover, the new intra-cavity $4 f$-telescope extensions greatly reduced the repetition rate of the oscillator down to $23 \mathrm{MHz}$, while single-pulse operation in the fundamental mode was maintained. When reducing the relative humidity level in the laser chamber, a strong effect on the mode-locked spectrum was observed, with the change in intra-cavity dispersion resulting in a measured Fourier-transform limited pulse duration of less than three optical cycles $\left(\tau_{p}=22 \mathrm{fs}\right)$. To the best of our knowledge, the pulse durations presented in this chapter are among the shortest reported for Cr:ZnS/ZnSe oscillators [64], and are the shortest that have been fully characterized in both amplitude and phase. 
Also, given the high peak power levels of up to $1 \mathrm{MW}$, generation of mid-infared radiation was demonstrated for the first time with the direct output of a Cr:ZnS/ZnSe oscillator. Notably, no additional amplification stage was involved in the setup - transferring the low-noise characteristics of the diode-pumped laser system directly into the mid-infrared region. With the technique of intra-pulse difference frequency generation and a ZGP crystal used as nonlinear medium, a comparably high opticalto-optical conversion efficiency of $5.7 \%$ was obtained for the spectral range between 4.5-14 $\mathrm{\mu m}$. The corresponding average power levels amounted to more than $20 \mathrm{~mW}$ when measured directly behind the $4.5 \mu \mathrm{m}$ long-pass filter, highlighting the enormous potential of direct oscillator-driven MIR generation. 



\section{CONCLUSION AND OUTLOOK}

In this thesis, diode-pumped few-cycle Cr:ZnS/ZnSe oscillators have been realized for the first time, with the oscillators' performance matching and even exceeding that of state-of-the-art systems based on mature fiber-laser-pumped technologies. The innovative combination of direct diode-pumping with ultrafast Kerr-lens modelocked $\mathrm{Cr}: \mathrm{ZnS} / \mathrm{ZnSe}$ technology led to highly stable, low-noise, and femtosecond output at wavelengths between $2 \mu \mathrm{m}$ to $3 \mu \mathrm{m}$, facilitating the direct generation of broadband mid-infrared spectra at the milliwatt-level. This last chapter serves to highlight the main experimental findings of this thesis and strives to showcase their tremendous potential for the rapidly growing field of laser-based molecular fingerprinting.

\subsection{Summary}

Generating broadband and coherent radiation in the mid-infrared range between $2.5 \mu \mathrm{m}$ and $20 \mu \mathrm{m}$ is of utmost importance for a plethora of applications, ranging from environmental monitoring $[245,314]$ to medical diagnostics $[315,316]$. Recent advancements have markedly benefited from the continuous development of ultrafast laser sources, where great interest is devoted to materials that can provide direct lasing in the MIR region. Solid-state bulk oscillators based on $\mathrm{Cr}^{2+}$-doped II-VI materials have proven to be reliable sources for generating few-cycle pulses in the $2 \mu \mathrm{m}$ to $3 \mu \mathrm{m}$ spectral range, and rare-earth-doped fiber lasers have been the pump sources of choice for building high-power systems. This thesis has showcased the enormous potential of bringing cutting-edge laser diode technology and powerful Kerr-lens mode-locked Cr:ZnS/ZnSe lasers together for the first time to help meet the growing demand for broadband, low-noise, and accessible table-top mid-IR sources. The main findings and results are briefly summarized in the following, highlighting the major breakthroughs and continuous refinements achieved during the development of three directly diode-pumped KLM systems (see table 6.1).

In chapter 2 , the theoretical foundations behind ultrashort pulse generation have been laid, with a focus on soliton pulse propagation and soliton mode-locking. Starting with the concept of mode-locking in a simple Fabry-Pérot laser resonator, the advantageous technique of Kerr-lens mode-locking (KLM) was described and analyzed in detail. Conditions under which fundamental solitons are formed were 
also discussed. Finally presented was the master equation of mode-locking which characterizes the complex interplay between the many effects governing the pulse evolution in a laser cavity, including self-amplitude modulation and the spectral filtering action of the gain medium.

In chapter 3, the favourable material and spectroscopic properties of chromiumdoped zinc sulfide and zinc selenide $\left(\mathrm{Cr}^{2+}: \mathrm{ZnS} / \mathrm{ZnSe}\right)$ were highlighted, drawing parallels to the well-known titanium sapphire crystals used in the near-infrared region $(<1 \mu \mathrm{m})$. Also, the challenges associated with direct diode-pumping and their effects on the generation of few-cycle pulses were addressed, particularly with regard to soft-aperture KLM. A sophisticated pump setup and resonator design finally led to the first realization of a directly diode-pumped Kerr-lens mode-locked $\mathrm{Cr}^{2+}$-doped II-VI oscillator (MIRanda1), pumped by the emission of a single-emitter laser diode. With a mode-locked output power of $500 \mathrm{~mW}$ at a repetition rate of $65 \mathrm{MHz}$ and pulse durations as short as $45 \mathrm{fs}$, a sixty-fold increase in peak power could be demonstrated over the previous record based on SESAM mode-locking. Moreover, noise measurements revealed an excellent amplitude noise performance of the mode-locked output, resulting in an integrated RMS relative intensity noise of less than $0.068 \%$ RMS $(20 \mathrm{~Hz}-1 \mathrm{MHz})$ for the frequency-doubled output.

In chapter 4 , the polarization-multiplexed combination of two single-emitter pump laser diodes resulted in a powerful second-generation system (MIRanda2), placing the performance of directly diode-pumped $\mathrm{Cr}^{2+}: \mathrm{ZnS} / \mathrm{ZnSe}$ laser systems on the same level as state-of-the-art fiber-laser-pumped counterparts. By evaluating the Kerr strength of the oscillator via nonlinear ABCD-matrix simulations and overcoming the challenges of higher average and intra-cavity peak power levels, mode-locked output powers of over $800 \mathrm{~mW}$ were recorded. Combined with the improved fine-tuning of the cavities' round-trip dispersion via dedicated dispersive optics, a further reduction in pulse duration down to four optical cycles $\left(\tau_{p}=34 \mathrm{fs}\right.$ ) was achieved. At a repetition frequency of $50 \mathrm{MHz}$, this resulted in an almost three-fold increase in peak power over MIRanda1, while the low amplitude noise performance of the Kerr-lens mode-locked oscillator was retained (0.014\% RMS for $20 \mathrm{~Hz}$ to $1 \mathrm{MHz}$ ).

In chapter 5 , the performance of directly diode-pumped $\mathrm{Cr}^{2+}$-doped II-VI laser oscillators was brought to a new level. A classic bow-tie cavity was realized for direct diode-pumping by implementing a novel pump focusing approach. Moreover, the incorporation of $4 \mathrm{f}$-telescope extensions into the resonator arms and a change of the focal spot diameter in the crystal allowed to operate the soft-aperture KLM laser system at very low repetition rates of only $23 \mathrm{MHz}$. Being pumped by the emission of two single-emitter laser diodes at up to 4.5 Watts of combined power, a peak power as high as $1 \mathrm{MW}$ was reached at pulse durations of $28 \mathrm{fs}$ and an 
equally low amplitude noise level as previously achieved (0.015\% RMS for $20 \mathrm{~Hz}$ to $1 \mathrm{MHz}$ ). To demonstrate the power of this third-generation system (MIRanda3), the generation of mid-infrared radiation using the oscillator's direct output was presented for the first time. With the output focused into a nonlinear optical crystal (ZGP), a multi-octave-spanning spectrum between $4.5 \mu \mathrm{m}$ to $14 \mu \mathrm{m}$ was detected. At optical-to-optical efficiencies of up to $5.7 \%$, the utilized technique of intra-pulse difference frequency generation can directly provide few-cycle pulses at the milliwatt-level, featuring intrinsic carrier-envelope phase stabilization.

\begin{tabular}{|c|c|c|c|}
\hline & MIRanda1 [209] & MIRanda2 [266] & MIRanda3 \\
\hline ML mechanism & Kerr-lens ML & Kerr-lens ML & Kerr-lens ML \\
\hline Gain medium & $\mathrm{Cr}: \operatorname{ZnSe}(5 \mathrm{~mm})$ & Cr:ZnS (5 mm) & Cr: $\operatorname{ZnS}(5 \mathrm{~mm})$ \\
\hline Pump diodes & $\operatorname{InP}(1 \mathrm{x})$ & $\operatorname{InP}(2 \mathrm{x})$ & $\operatorname{InP}(2 \mathrm{x})$ \\
\hline$P_{\text {pump }}$ & $3 \mathrm{~W}$ & $5 \mathrm{~W}$ & $4.5 \mathrm{~W}$ \\
\hline$\lambda_{\text {pump }}$ & $1.65 \mu \mathrm{m}$ & $1.65 \mu \mathrm{m}$ & $1.65 \mu \mathrm{m}$ \\
\hline$P_{C W}$ & $700 \mathrm{~mW}$ & $1200 \mathrm{~mW}$ & $1100 \mathrm{~mW}$ \\
\hline$P_{M L}$ & $500 \mathrm{~mW}$ & $800 \mathrm{~mW}$ & $720 \mathrm{~mW}$ \\
\hline$\tau_{0}$ & $45 \mathrm{fs}$ & $34 \mathrm{fs}$ & $28 \mathrm{fs}$ \\
\hline$\nu_{r e p}$ & $64.7 \mathrm{MHz}$ & $49.9 \mathrm{MHz}$ & $22.9 \mathrm{MHz}$ \\
\hline OC ratio & $34 \%$ & $25 \%$ & $25 \%$ \\
\hline RIN & $0.068 \%^{(\star)}$ & $0.014 \%$ & $0.015 \%$ \\
\hline$E_{0}$ & $7.7 \mathrm{~nJ}$ & $16 \mathrm{~nJ}$ & $31 \mathrm{~nJ}$ \\
\hline$P_{0}$ & $151 \mathrm{~kW}$ & $415 \mathrm{~kW}$ & $988 \mathrm{~kW}$ \\
\hline
\end{tabular}

Table 6.1: Comparison of the laser performance of the three directly diode-pumped $\mathrm{Cr}^{2+}$ doped II-VI laser systems presented in this thesis. The integrated RMS relative intensity noise (RIN) was calculated between $20 \mathrm{~Hz}$ and $1 \mathrm{MHz}$ and measured in mode-locked laser operation. $\left.{ }^{(}\right)$Value corresponds to the frequency-doubled laser output.

\subsection{A New Ultrafast Workhorse for Spectroscopic Applications?}

To answer this question, it is worthwhile to examine the results achieved from an application-oriented perspective. Starting with the output characteristics provided by the three directly diode-pumped Cr:ZnS/ZnSe oscillators, a highly-promising low-noise performance of the few-cycle laser output has been shown. For applications 
such as Fourier-transform-based spectroscopy [317], it is the laser's relative intensity noise (RIN) that constitutes the main limiting factor on the overall detection sensitivity. Besides the dynamic range of the detection setup as well as the detector noise and shot noise, laser RIN sets the ultimate limit to the signal-to-noise ratio at source powers approaching the $\mathrm{mW}$-range [317]. With the technique of direct diode-pumping and the resulting low-noise laser operation, we are confident that the specificity and sensitivity achieved with electric-field resolved measurements [40] can be pushed to a new level. Moreover, when increasing the peak power of diode-pumped $\mathrm{Cr}: \mathrm{ZnS} / \mathrm{ZnSe}$ lasers to the $1 \mathrm{MW}$-level, multi-octave-spanning mid-infrared generation between $4.5 \mu \mathrm{m}$ to $14 \mu \mathrm{m}$ is feasible via oscillator-driven intra-pulse DFG in a nonlinear crystal. Not only are the low-noise properties of the driving laser directly transferred into the MIR region, this alignment-insensitive single-beam geometry also allows to generate few-cycle MIR pulses with intrinsic carrier-envelope phase (CEP) stability [39].

The presented results already lay the foundation for advancing the field of infrared spectroscopy, but there are aspects that can be further improved in the future. In particular, the technique of electro-optic sampling greatly benefits from shorter sampling pulses. EOS probes the birefringence of the crystal that has been modulated by the electric field of the mid-infrared pulse, with the measurements being performed as a function of the delay between both pulses. However, the recorded EOS signal on the short-wavelength side is limited by the duration of the probe pulse [318], and broader frequencies spans are only detectable for decreasing sampling pulse durations. Typically, it is said that the probe pulse has to be shorter than a half-cycle of the oscillating field to be observed [49], and a common way to achieve this is to split off part of the oscillator output for further nonlinear spectral broadening and compression [315, 318]. In nonlinear media, the propagation of few-cycle pulses is influenced by the $\chi^{(3)}$-effects of self-focusing and self-phase modulation, and a proper control of the material parameters can result in a significant reduction in pulse duration. Over the past few years, fiber-based [73] approaches as well as bulk compression schemes have already been successfully demonstrated in our group [85, 319, 320] and more experimental research is currently undertaken with $\mathrm{Cr}: \mathrm{ZnS} / \mathrm{ZnSe-based} \mathrm{systems.}$

In addition to shorter pulses, the carrier-envelope phase of the oscillator can be actively stabilized, such that new MIR frequencies - converted using methods other than non-cascaded IDFG [33] — will also share this important property. With the aforementioned shortening of pulse durations and the corresponding spectral broadening, traditional carrier-envelope-offset detection schemes based on interference beatings between the fundamental spectrum and its second-harmonic signal can be employed [274]. Alternatively, a recently reported scheme for Cr:ZnS/ZnSe-based master oscillator power amplifier (MOPA) systems [37] utilizing the harmonics- 
enhanced supercontinuum generation observed in polycrystalline $\mathrm{ZnS}$ and $\mathrm{ZnSe}$ [321] could also be implemented. By detecting these beatings between adjacent harmonics and incorporating phase-locked loop controllers, ultrabroadband and CEP-stabilized mid-infrared waveforms can finally be realized.

Therefore, one can confidently say that diode-pumped few-cycle $\mathrm{Cr}^{2+}$-doped II-VI technology has enormous potential to bring us closer towards the ultimate limits of mid-IR spectroscopy. The dramatically simplified design architecture for mid-infrared generation, combined with high efficiencies, intrinsic low-noise, and direct few-cycle pulse generation, is already competitive with currently used systems, and will potentially render the complex $1 \mu \mathrm{m}$-based laser frontends obsolete.

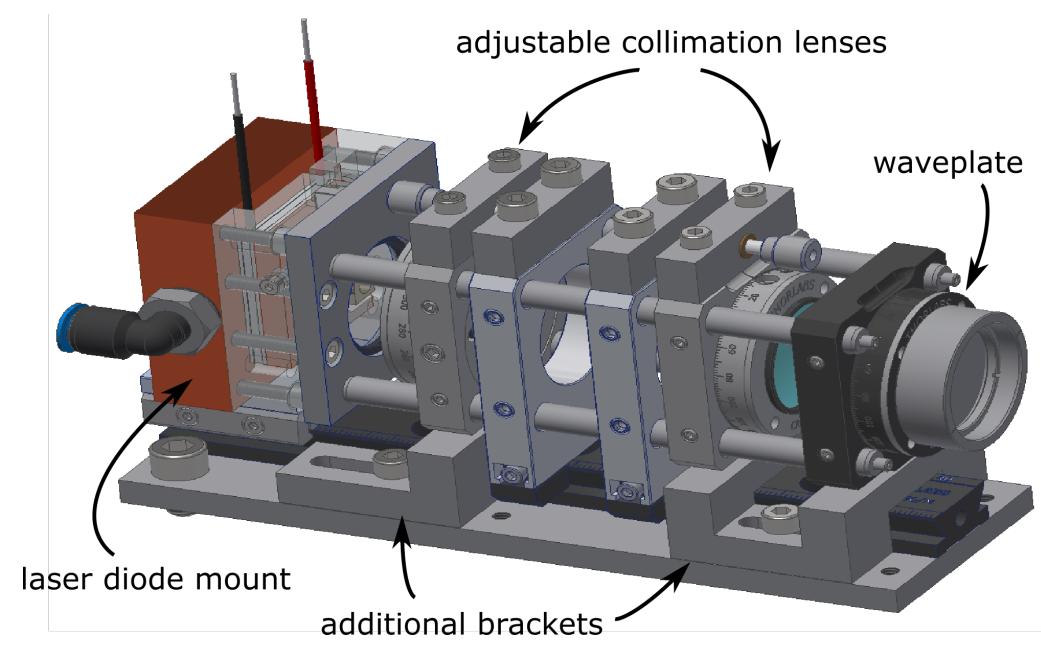

Fig. 6.1: $3 \mathrm{D}$ mechanical design of the new laser diode collimation unit. The picture was kindly provided by our laser engineer Aleksandar Sebesta.

\section{What's Next? — Prototype Development for Mid-IR Applications}

Based on the experimental findings and achievements presented in this thesis, a laser system highly optimized for stability, compactness and low-maintenance operation (see 3D designs in figures 6.1 and 6.2) is currently being developed at our research laboratory for upcoming spectroscopic measurements [40]. It will incorporate two pump laser diodes and a $50 \mathrm{MHz}$-oscillator structure - very similar to MIRanda2 - and is meant to deliver more than $500 \mathrm{~mW}$ of average power at pulse durations shorter than five optical cycles $\left(\tau_{p}<40 \mathrm{fs}\right)$. Moreover, the airtight and vacuumcompatible chamber environment eliminates effects such as air turbulences, dust particles and water absorption to affect the laser performance, and allows for its 
flexible deployment in different laboratories. Features such as live monitoring of laser parameters further help to identify potential problems before they aggravate. A first prototype is currently set up by our laser engineer, with a picture of the real laser system shown in figure 6.2. After all those months and years of work, it is gratifying to see how the basic experimental research performed in this thesis starts to enter a new phase, heading to explore the weakest molecular signatures in the entire fingerprint region via field-resolved spectroscopy.

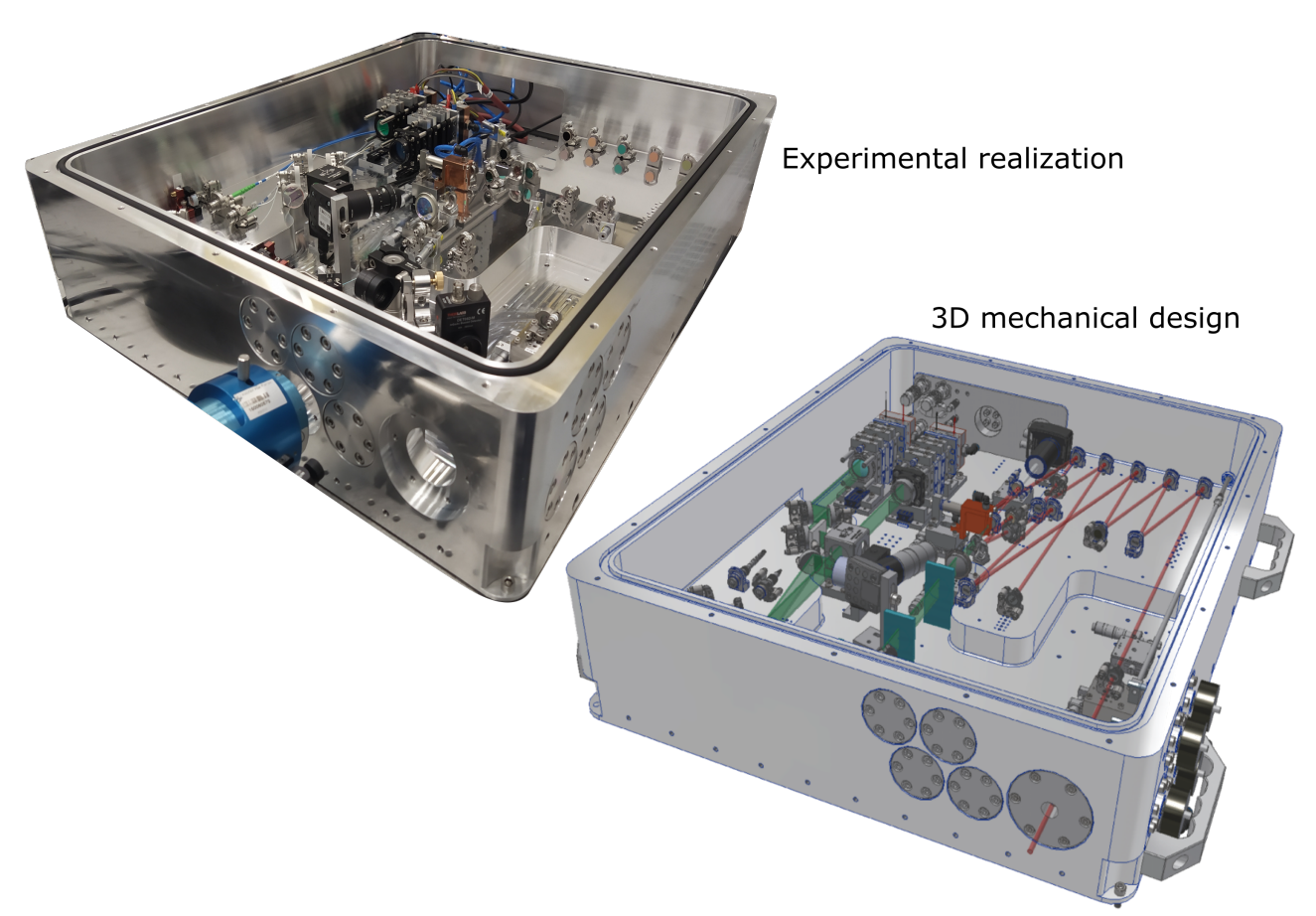

Fig. 6.2: 3D mechanical design of the laser prototype and its experimental realization. The laser design is based on the second diode-pumped system MIRanda2. The pictures were kindly provided by our laser engineer Aleksandar Sebesta.

\subsection{Closing Remarks}

The presented few-cycle $\mathrm{Cr}^{2+}$-doped II-VI laser oscillators - combined with the intra-pulse difference frequency generation scheme - herald a new era of ultrafast mid-infrared coherent light sources and render directly diode-pumped Cr:ZnS/ZnSe oscillators as one of the most promising driving sources for the emerging fields of nano-imaging [13-15] as well as time-domain [14, 16] and frequency comb spectroscopy [17-19]. Moreover, with their unique capability to provide low-noise and ultrabroadband mid-infrared radiation already at the milliwatt level, the directly 
diode-pumped Cr:ZnS/ZnSe systems open up new avenues into the study of complex biological systems in the entire molecular fingerprint region [40]. The exploration phase has certainly just begun, and there are many more specimens that are waiting to be examined by ultrafast laser technology.

Pushing the frontiers of state-of-the-art solid-state laser systems for advancements in MIR applications was the key focus and main driver of this $\mathrm{PhD}$ thesis. From the knowledge gained and the breakthroughs achieved, the realization of a compact and low-noise coherent mid-infrared source is now one step closer. Nonetheless, the philosophy behind this work will remain unchanged:

Advancing a Just Cause. 



\section{A. APPENDIX}

\section{A.1 List of Publications}

\section{Peer-Reviewed Journal Articles (First Author):}

- Nathalie Nagl, Ka Fai Mak, Qing Wang, Vladimir Pervak, Ferenc Krausz, and Oleg Pronin, "Efficient femtosecond mid-infrared generation based on a Cr:ZnS oscillator and step-index fluoride fibers," Opt. Lett. 44, 2390-2393 (2019)

The author designed and set up the fiber-based nonlinear spectral broadening unit, prepared the fibers, performed the measurements, analyzed the experimental data, and prepared the manuscript together with K. F. Mak.

- Nathalie Nagl, Sebastian Gröbmeyer, Vladimir Pervak, Ferenc Krausz, Oleg Pronin, and Ka Fai Mak, "Directly diode-pumped, Kerr-lens mode-locked, few-cycle Cr:ZnSe oscillator," Opt. Express 27, 24445-24454 (2019)

The author designed and built the laser system, performed the measurements, analyzed the experimental data, and prepared the manuscript together with $K$. F. Mak.

- Nathalie Nagl, Ka Fai Mak et al., "Broadband mid-infrared generation driven by a directly diode-pumped ultrafast Cr:ZnS oscillator at high peak power" (in preparation)

The author designed and built the laser system, set up the MIR generation unit, performed the measurements, and analyzed the experimental data. The manuscript is prepared together with K. F. Mak.

\section{Peer-Reviewed Journal Articles (Co-Author):}

- Jinwei Zhang, Ka Fai Mak, Nathalie Nagl, Marcus Seidel, Dominik Bauer, Dirk Sutter, Vladimir Pervak, Ferenc Krausz, and Oleg Pronin, "Multi-mW, few-cycle mid-infrared continuum spanning from 500 to 2250 cm-1," Light Sci. Appl. 7, 17180 (2017) 
The author built the pulse characterization unit (SHG-FROG), assisted in the pulse duration measurements, revised and discussed the manuscript.

- Qing Wang, Jinwei Zhang, Alexander Kessel, Nathalie Nagl, Vladimir Pervak, Oleg Pronin, and Ka Fai Mak, "Broadband mid-infrared coverage (2-17 $\mu \mathrm{m})$ with few-cycle pulses via cascaded parametric processes," Opt. Lett. 44, 2566-2569 (2019)

The author built the pulse characterization unit (SHG-FROG), assisted in the pulse duration measurements, revised and discussed the manuscript.

- Thomas P. Butler, Nikolai Lilienfein, Jia Xu, Nathalie Nagl, Christina Hofer, Daniel Gerz, Ka Fai Mak, Christian Gaida, Tobias Heuermann, Martin Gebhardt, Jens Limpert, Ferenc Krausz and Ioachim Pupeza, "Multi-octave spanning, Watt-level ultrafast mid-infrared source," JPhys Photonics 1, 044006 (2019)

The author performed preliminary proof of concept experiments with the $Z B L A N$ fibers, assisted in the fiber parameter selection as well as the fiber preparation, revised and discussed the manuscript.

\section{Conference Talks:}

- Nathalie Nagl, Ka Fai Mak, Vladimir Pervak, Ferenc Krausz, and Oleg Pronin, "200 mW-level, few-opticle-cycle tunable femtosecond mid-infrared source based on a Cr:ZnS oscillator and step-index fluoride fibers," in 2018 8th EPS-QEOD Europhoton Conference, paper FrM1.3 (2018)

- Nathalie Nagl, Sebastian Gröbmeyer, Vladimir Pervak, Oleg Pronin, Ka Fai Mak, and Ferenc Krausz, "Directly Diode-Pumped, Kerr-Lens Mode-Locked Cr:ZnSe Oscillator," in 2019 Conference on Lasers and Electro-Optics Europe and European Quantum Electronics Conference, OSA Technical Digest (Optical Society of America, 2019), paper CF-2.2 (2019)

- Nathalie Nagl, Sebastian Gröbmeyer, Markus Pötzlberger, Vladimir Pervak, Ferenc Krausz, and Ka Fai Mak, "Directly Diode-Pumped Few-Optical-Cycle $\mathrm{Cr}: Z n S$ Laser at $800 \mathrm{~mW}$ of Average Power," in 2020 Conference on Lasers and Electro-Optics Europe and European Quantum Electronics Conference, OSA Technical Digest (Optical Society of America, 2020), paper SF3H.5 (2020) 


\section{A.2 Data Archiving}

The experimental raw data, evaluation files, and original figures can be found on the Data Archive Server of the Laboratory for Attosecond Physics at the Max Planck Institute of Quantum Optics:

//AFS/ipp-garching.mpg.de/mpq/lap/publication_archive.

All files are organized in a folder structure that is in accordance with the numbering and the structure of the thesis. Each folder contains figures in .png format, raw data and Python scripts if needed. Also, a ReadMe.txt file is added to every folder, providing further explanations and information on the presented data. 



\section{REFERENCES}

[1] A. Einstein. "Zur Quantentheorie der Strahlung". In: Physikalische Zeitschrift 18 (1917), pages 121-128.

[2] T. H. Maiman. "Stimulated Optical Radiation in Ruby". In: Nature 187 (1960), pages 493-494.

[3] P. P. Sorokin and M. J. Stevenson. "Stimulated Infrared Emission from Trivalent Uranium". In: Physical Review Letters 5.12 (1960), pages 557-559.

[4] K. C. Cossel, E. M. Waxman, I. A. Finneran, G. A. Blake, J. Ye, and N. R. Newbury. "Gas-phase broadband spectroscopy using active sources: progress, status, and applications". In: Journal of the Optical Society of America B 34.1 (2017), pages 104-129.

[5] J. Li, W. Chen, and H. Fischer. "Quantum Cascade Laser Spectrometry Techniques: A New Trend in Atmospheric Chemistry". In: Applied Spectroscopy Reviews 48.7 (2013), pages 523-559.

[6] P. R. Griffiths and J. A. de Haseth. Fourier Transform Infrared Spectrometry. 2nd edition. John Wiley \& Sons, Inc., 2006.

[7] Z. Movasaghi, S. Rehman, and I. Rehman. "Fourier Transform Infrared (FTIR) Spectroscopy of Biological Tissues". In: Applied Spectroscopy Reviews 43.2 (2008), pages 134-179.

[8] A. B. Eldin. "Wide Spectra of Quality Control". In: edited by I. Akyar. IntechOpen, 2011. Chapter Near Infra Red Spectroscopy, pages 237-248.

[9] E. Gray et al. "Health economic evaluation of a serum-based blood test for brain tumour diagnosis: exploration of two clinical scenarios". In: BMJ Open 8.5 (2018), pages $1-9$.

[10] C. Petibois, G. Deleris, M. Piccinini, M. Cestelli-Guidi, and A. Marcelli. "A bright future for synchrotron imaging". In: Nature Photonics 3 (2009), page 179 .

[11] M. C. Martin, U. Schade, P. Lerch, and P. Dumas. "Recent applications and current trends in analytical chemistry using synchrotron-based Fouriertransform infrared microspectroscopy". In: Trends in Analytical Chemistry 29.6 (2010), pages 453-463.

[12] A. Marcelli and G. Cinque. "Infrared synchrotron radiation beamlines: high brilliance tools for IR spectromicroscopy". In: RSC Analytical Spectroscopy Series (2011), pages 67-104. 
[13] I. Amenabar, S. Poly, M. Goikoetxea, W. Nuansing, P. Lasch, and R. Hillenbrand. "Hyperspectral infrared nanoimaging of organic samples based on Fourier transform infrared nanospectroscopy". In: Nature Communications 8.14402 (2017).

[14] M. Eisele et al. "Ultrafast multi-terahertz nano-spectroscopy with sub-cycle temporal resolution". In: Nature Photonics 8 (2014), pages 841-845.

[15] L. Wang and X. G. Xu. "Scattering-type scanning near-field optical microscopy with reconstruction of vertical interaction". In: Nature Communications 6 (2015), page 8973.

[16] A. A. Lanin, A. A. Voronin, A. B. Fedotov, and A. M. Zheltikov. "Timedomain spectroscopy in the mid-infrared". In: Scientific Reports 4 (2014), page 6670 .

[17] N. Piqué and T. W. Hänsch. "Frequency comb spectroscopy". In: Nature Photonics 13 (2019), pages 146-157.

[18] A. Schliesser, N. Picqué, and T. W. Hänsch. "Mid-infrared frequency combs". In: Nature Photonics 6 (2012), pages 440-449.

[19] I. Coddington, N. Newbury, and W. Swann. "Dual-comb spectroscopy". In: Optica 3.4 (2016), pages 414-426.

[20] R. P. S. M. Lobo et al. "Photoinduced time-resolved electrodynamics of superconducting metals and alloys". In: Physical Review B 72.024510 (2005).

[21] R. P. S. M. Lobo, J. D. LaVeigne, D. H. Reitze, D. B. Tanner, and G. L. Carr. "Subnanosecond, time-resolved, broadband infrared spectroscopy using synchrotron radiation". In: Review of Scientific Instruments 73.1 (2002).

[22] C. A. D. Roeser and E. Mazur. "Frontiers of Optical Spectroscopy". In: edited by B. Di Bartolo and O. Forte. Springer, 2005. Chapter Light-Matter Interactions on the Femtosecond Time Scale, pages 29-54.

[23] P. Dumas, F. Polack, B. Lagarde, O. Chubar, J. L. Giorgetta, and S. Lefrançois. "Synchrotron infrared microscopy at the French Synchrotron Facility SOLEIL". In: Infrared Physics \&s Technology 49 (2006), pages 152160.

[24] M. S. Vitiello, G. Scalari, B. Williams, and P. De Natale. "Quantum cascade lasers: 20 years of challenges". In: Optics Express 23.4 (2015), pages 51675182.

[25] B. G. Lee et al. "Widely tunable single-mode quantum cascade laser source for mid-infrared spectroscopy". In: Applied Physics Letters 91.23 (2007), page 231101. 
[26] V. Petrov. "Parametric down-conversion devices: The coverage of the midinfrared spectral range by soild-state laser sources". In: Optical Materials 34.3 (2012), pages 536-554.

[27] J. Peng. "Developments of mid-infrared optical parameteric oscillators for spectroscopic sensing: a review". In: Optical Engineering 53.6 (2014), page 061613.

[28] F. Keilmann and S. Amarie. "Mid-infrared frequency comb spanning an octave based on an Er fiber laser and difference-frequency generation". In: 2013 Conference on Lasers and Electro-Optics - International Quantum Electronics Conference. Optical Society of America, 2013, CC.P.2.

[29] T. Steinle, F. Mörz, A. Steinmann, and H. Giessen. "Ultra-stable high average power femtosecond laser system tunable from 1.33 to $20 \mu \mathrm{m}$ ". In: Optics Letters 41.21 (2016), pages 4863-4866.

[30] V. O. Smolski, S. Vasilyev, P. G. Schunemann, S. B. Mirov, and K. L. Vodopyanov. "Cr:ZnS laser-pumped subharmonic GaAs optical parameteric oscillator with the spectrum spanning 3.6-5.6 $\mu \mathrm{m}$ ". In: Optics Letters 40.12 (2015), pages 2906-2908.

[31] A. Fix et al. "Optical parametric oscillators and amplifiers for airborne and spaceborne active remote sensing of CO2 and CH4". In: Lidar Technologies, Techniques, and Measurements for Atmospheric Remote Sensing VII. Edited by U. N. Singh and G. Pappalardo. Volume 8182. SPIE, 2011, pages 28-37.

[32] C. Gaida et al. "Watt-scale super-octave mid-infrared intrapulse difference frequency generation". In: Light:Science 8 Applications 7.1 (2018), page 94.

[33] Q. Wang et al. "Broadband mid-infrared coverage (2-17 $\mu \mathrm{m})$ with few-cycle pulses via cascaded parametric processes". In: Optics Letters 44.10 (2019), pages 2566-2569.

[34] Q. Wang et al. "Highly efficient broadband mid-infrared generation (2.8$12.5 \mu \mathrm{m}$ ) based on a compact Cr:ZnS laser". In: 2019 Conference on Lasers and Electro-Optics Europe and European Quantum Electronics Conference. CF-7.5. IEEE, 2019.

[35] J. Zhang et al. "Multi-mW, few-cycle mid-infrared continuum spanning from 500 to $2250 \mathrm{~cm}^{-1}$ ". In: Light: Science and Applications 7 (2018), page 17180.

[36] J. Zhang, K. Fritsch, Q. Wang, F. Krausz, K. Mak, and O. Pronin. "Intrapulse difference-frequency generation of mid-infrared (2.7 -20 $\mu \mathrm{m})$ by random quasi-phase-matching". In: Optics Letters 44.12 (2019), pages 2986-2989.

[37] S. Vasilyev, I. Moskalev, M. Mirov, S. Mirov, and V. Gapontsev. "MultiWatt Mid-Ir Femtosecond Polycrystalline $\mathrm{Cr}^{2+}: \mathrm{ZnS}$ and $\mathrm{Cr}^{2+}: \mathrm{ZnSe}$ Laser Amplifiers With the Spectrum Spanning 2.0-2.6 um”. In: Optical Society of America 24.2 (2016), pages 1616-1623. 
[38] A. Baltuška, T. Fuji, and T. Kobayashi. "Controlling the Carrier-Envelope Phase of Ultrashort Light Pulses with Optical Parametric Amplifiers". In: Physical Review Letters 88.13 (2002), page 133901.

[39] I. Pupeza et al. "High-power sub-two-cycle mid-infrared pulses at $100 \mathrm{MHz}$ repetition rate". In: Nature Photonics 9 (2015), pages 721-724.

[40] I. Pupeza et al. "Field-resolved infrared spectroscopy of biological systems". In: Nature 577.7788 (2020), pages 52-59.

[41] Z. Bacsik, J. Mink, and G. Keresztury. "FTIR Spectroscopy of the Atmosphere. I. Principles and Methods". In: Applied Spectroscopy Reviews 39.3 (2004), pages 295-363.

[42] A. Barth. "Infrared spectroscopy of proteins". In: Biochimica et Biophysica Acta (BBA) - Bioenergetics 1767.9 (2007), pages 1073-1101.

[43] C. Petibois and G. Deleris. "Chemical mapping of tumor progression by FTIR imaging: towards molecular histopathology". In: Trends in Biotechnology 24.10 (2006), pages 455-462.

[44] A. Sala et al. "Biofluid diagnostics by FTIR spectroscopy: A platform technology for cancer detection". In: Cancer Letters 477 (2020), pages 122-130.

[45] P. Jacquinot. "New developments in interference spectroscopy". In: Reports on Progress in Physics 23.1 (1960), pages 267-312.

[46] P. Connes. "Astronomical Fourier Spectroscopy". In: Annual Review of Astronomy and Astrophysics 8 (1970), pages 209-230.

[47] F. K. Tittel, D. Richter, and A. Fried. "Solid-State Mid-Infrared Laser Sources". In: edited by I. T. Sorokina and K. Vodopyanov. Springer, 2003. Chapter 11, pages 445-497.

[48] T. W. Hänsch. "Nobel Lecture: Passion for precision". In: Reviews of Modern Physics 78.4 (2006), pages 1297-1309.

[49] S. Keiber et al. "Electro-optic sampling of near-infrared waveforms". In: Nature Photonics 10 (2016), pages 159-162.

[50] F. Mörz et al. "Nearly diffraction limited FTIR mapping using an ultrastable broadband femtosecond laser tunable from 1.33 to 8 um". In: Optics Express 25.26 (2017), pages 32355-32363.

[51] S. A. Diddams, L. Hollberg, and V. Mbele. "Molecular fingerprinting with the resolved modes of a femtosecond laser frequency comb". In: Nature 445 (2007), pages 627-630.

[52] W. Sibbett, A. A. Lagatsky, and C. T. A. Brown. "The development and application of femtosecond laser systems". In: Optics Express 20.7 (2012), pages 6989-7001. 
[53] E. Sorokin, I. T. Sorokina, J. Mandon, G. Guelachvili, and N. Picqué. "Sensitive multiplex spectroscopy in the molecular fingerprint $2.4 \mathrm{\mu m}$ region with a $\mathrm{Cr}^{2+}:$ ZnSe femtosecond laser". In: Optics Express 15.25 (2007), pages 1654016545.

[54] F. Krausz and M. Ivanov. "Attosecond physics". In: Reviews of Modern Physics 81.1 (2009), pages 163-234.

[55] M. DiDomenico. "Small-Signal Analysis of Internal (Coupling-Type) Modulation of Lasers". In: Journal of Applied Physics 35.10 (1964), pages 28702876.

[56] L. E. Hargrove, R. L. Fork, and M. A. Pollack. "Locking of He-Ne Laser Modes Induced by Synchronous Intracavity Modulation". In: Applied Physics Letters 5.1 (1964), pages 4-5.

[57] U. Keller, D. A. B. Miller, G. D. Boyd, T. H. Chiu, J. F. Ferguson, and M. T. Asom. "Solid-state low-loss intracavity saturable absorber for Nd:YLF lasers: an antiresonant semiconductor Fabry-Perot saturable absorber". In: Optics Letters 17.7 (1992), pages 505-507.

[58] D. E. Spence, P. N. Kean, and W. Sibbett. "60-fsec pulse generation from a self-mode-locked Ti:sapphire laser". In: Optics Letters 16.1 (1991), pages 4244 .

[59] F. Krausz et al. "Femtosecond Solid-State Lasers". In: IEEE Journal of Quantum Electronics 28.10 (1992), pages 2097-2122.

[60] U. Keller. "Recent developments in compact ultrafast lasers". In: Nature 424 (2003), pages 831-838.

[61] R. Ell et al. "Generation of 5-fs pulses and octave-spanning spectra directly from a Ti:sapphire laser". In: Optics Letters 26.6 (2001), pages 373-375.

[62] P. C. Wagenblast et al. "Generation of sub-10-fs pulses from a Kerr-lens mode-locked $\mathrm{Cr}^{3+}$ :LiCAF laser oscillator by use of third-order dispersioncompensating double-chirped mirrors". In: Optics Letters 27.19 (2002), pages $1726-1728$.

[63] C. Chudoba et al. "All-solid-state Cr:forsterite laser generating 14-fs pulses at 1.3 um". In: Optics Letters 26.5 (2001), pages 292-294.

[64] S. Mirov et al. "Frontiers of mid-IR lasers based on transition metal doped chalcogenides". In: IEEE Journal of Selected Topics in Quantum Electronics 24.5 (2018), pages 1-29.

[65] F. X. Kärtner, editor. Few-cycle laser pulse generation and its applications. 95. Springer, 2004.

[66] Simon Sinek. The Infinite Game. Portfolio/Penguin, 2019. 
[67] I. T. Sorokina and K. L. Vodopyanov. Solid-State Mid-Infrared Laser Sources. Volume 89. Topics in Applied Physics. Springer-Verlag, 2003.

[68] A. Godard. "Infrared (2-12 $\mu \mathrm{m})$ Solid-State Laser Sources: A Review". In: Comptes Rendus Physique 8.10 (2007), pages 1100-1128.

[69] J. Zhang et al. "High-Power, High-Efficiency Tm:YAG and Ho:YAG ThinDisk Lasers". In: Laser \& Photonics Reviews 12.3 (2018), page 1700273.

[70] C. Gaida, M. Gebhardt, T. Heuermann, F. Stutzki, C. Jauregui, and J. Limpert. "Ultrafast thulium fiber laser system emitting more than $1 \mathrm{~kW}$ of average power". In: Optics Letters 43.23 (2018), pages 5853-5856.

[71] J. Zhang, K. Mak, and O. Pronin. "Kerr-lens mode-locked $2 \mu \mathrm{m}$ thin-disk lasers". In: IEEE Journal of Selected Topics in Quantum Electronics 24.5 (2018).

[72] P. Li, A. Ruehl, U. Grosse-Wortmann, and I. Hartl. "Sub-100 fs passively mode-locked holmium-doped fiber oscillator operating at $2.06 \mu \mathrm{m}$ ". In: Optics Letters 39.24 (2014), pages 6859-6862.

[73] N. Nagl, K. Mak, Q. Wang, V. Pervak, F. Krausz, and O. Pronin. "Efficient femtosecond mid-infrared generation based on a Cr:ZnS oscillator and stepindex fluoride fibers". In: Optics Letters 44.10 (2019), pages 2390-2393.

[74] A. Giesen, H. Hügel, A. Voss, K. Wittig, U. Brauch, and H. Opower. "Scalable concept for diode-pumped high-power solid-state lasers". In: Applied Physics B 58 (1994), pages 365-372.

[75] C. J. Saraceno, D. Sutter, T. Metzger, and M. A. Ahmed. "The amazing progress of high-power ultrafast thin-disk lasers". In: Journal of the European Optical Society-Rapid Publications 15.15 (2019).

[76] G. Renz, J. Speiser, A. Giesen, I. T. Sorokina, and E. Sorokin. "Cr:ZnSe thin disk cw laser". In: Solid State Lasers XXII: Technology and Devices. Volume 8599. SPIE, 2013, pages 310-316.

[77] I. Moskalev et al. "140 W Cr:ZnSe laser system". In: Optics Express 24.18 (2016), pages 21090-21104.

[78] A. Hildenbrand et al. "Laser damage of the nonlinear crystals $\mathrm{CdSiP}_{2}$ and $\mathrm{ZnGeP}_{2}$ studied with nanosecond pulses at 1064 and $2090 \mathrm{~nm}$ ". In: Optical engineering 53.12 (2014), page 122511.

[79] M. Seidel et al. "Multi-watt, multi-octave, mid-infrared femtosecond source". In: Science Advances 4.4 (2018).

[80] B.-H. Chen, T. Nagy, and P. Baum. "Efficient middle-infrared generation in $\mathrm{LiGaS}_{2}$ by simultaneous spectral broadening and difference-frequency generation". In: Optics Letters 43.8 (2018), pages 1742-1745. 
[81] R. Boyd. Nonlinear Optics. 3rd edition. Academic Press, 2008.

[82] L. A. Vazquez-Zuniga and Y. Jeong. "Wavelength-Tunable, PaPassive ModeLocked Erbium Doped Fiber Master-Oscillator Incorporating a Semiconductor Saturable Absorber Mirror". In: Journal of the Optical Society of Korea 17.2 (2013), pages 117-129.

[83] I. T. Sorokina, V. V. Dvoyrin, N. Tolstik, and E. Sorokin. "Mid-IR Ultrashort Pulsed Fiber-Based Lasers". In: IEEE Journal of Selected Topics in Quantum Electronics 20.5 (2014).

[84] V. Petrov. "Frequency down-conversion of solid-state laser sources to the mid-infrared spectral range using non-oxide nonlinear crystals". In: Progress in Quantum Electronics 42 (2015), pages 1-106.

[85] K. Fritsch, M. Pötzlberger, V. Pervak, J. Brons, and O. Pronin. "All-solidstate multipass spectral broadening to sub-20 fs". In: Optics Letters 43.19 (2018), pages 4643-4646.

[86] Orazio Svelto. Principles of Lasers. 5th edition. Springer, 2009.

[87] W. Koechner. Solid-State Laser Engineering. Springer, 2006.

[88] Anthony E. Siegman. Lasers. University Science Books, 1986.

[89] H. J. Eichler, J. Eichler, and O. Lux. Lasers - Basics, Advances and Applications. 1st edition. Springer, 2018.

[90] A. M. Weiner. Ultrafast Optics. 1st edition. John Wiley \& Sons, 2009.

[91] W. Koechner and M. Bass. "Solid-State Lasers". In: Advanced Texts in Physics. Springer New York, 2003. Chapter Optical Resonator, pages 149186.

[92] D. J. Kuizenga and A. E. Siegman. "FM and AM mode locking of the homogeneous laser - Part I: Theory". In: IEEE Journal of Quantum Electronics 6.11 (1970), pages 694-708.

[93] U. Keller et al. "Semiconductor Saturable Absorber Mirrors (SESAM's) for Femtosecond to Nanosecond Pulse Generation in Solid-State Lasers". In: IEEE Journal of Selected Topics in Quantum Electronics 2.3 (1996), pages $435-453$.

[94] U. Keller, G. W. 'tHooft, W. H. Knox, and J. E. Cunningham. "Femtosecond pulses from a continuously self-starting passively mode-locked Ti:sapphire laser". In: Optics Letters 16.13 (1991), pages 1022-1024.

[95] U. Morgner et al. "Sub-two-cycle pulses from a Kerr-lens mode-locked Ti:sapphire laser". In: Optics Letters 24.6 (1999), pages 411-413. 
[96] D. H. Sutter et al. "Semiconductor saturable-absorber mirror-assisted Kerrlens mode-locked Ti:sapphrie laser producing pulses in the two-cycle regime". In: Optics Letters 24.9 (1999), pages 631-633.

[97] M. Dussauze and T. Cardinal. "Springer Handbook of Glass". In: edited by J. D. Musgraves, J. Hu, and L. Calvez. Springer, 2019. Chapter Nonlinear optical properties of glass, pages 193-225.

[98] L. Prussel. "Ab-initio description of optical nonlinear properties of semiconductors under an electrostatic field". PhD thesis. Université Paris-Saclay, 2017.

[99] C. Li. "Nonlinear Optics - Principles and Applications". In: 1st edition. Springer-Verlag, 2017. Chapter 2, pages 23-50.

[100] V. Degiorgio and I. Cristiani. Photonics - A Short Course. Undergraduate Lecture Notes in Physics. Springer-Verlag, 2014.

[101] J. Herrmann. "Theory of Kerr-lens mode locking: role of self-focusing and radially varying gain". In: Journal of the Optical Society of America B 11.3 (1994), pages 498-512.

[102] M. Piché and F. Salin. "Self-mode locking of solid-state lasers without apertures". In: Opt. Lett. 18.13 (1993), pages 1041-1043.

[103] V. Magni, G. Cerullo, and S. De Silvestri. "Closed form gaussian beam analysis of resonators containing a Kerr medium for femtosecond lasers". In: Optics Communications 101.5-6 (1993), pages 365-370.

[104] S. Yefet and A. Pe'er. "A Review of Cavity Design for Kerr Lens Mode-Locked Solid-State Lasers". In: Applied Sciences 3.4 (2013), pages 694-724.

[105] G. Fibich and A. L. Gaeta. "Critical power for self-focusing in bulk media and in hollow waveguides". In: Optics Letters 25.5 (2000), pages 335-337.

[106] M. Remoissenet. Waves called Solitons. 2nd edition. Springer, 1996.

[107] Govind P. Agrawal. Nonlinear Fiber Optics. 4th edition. Elsevier (Singapore) Pte Ltd, 2009.

[108] J. A. Buck. Fundamentals of Optical Fibers. 2nd edition. Wiley-Interscience, 2004.

[109] M. Debenham. "Refractive indices of zinc sulfide in the 0.405-13 $\mu \mathrm{m}$ wavelength range". In: Applied Optics 23.14 (1984), pages 2238-2239.

[110] A. Major, J. S. Aitchison, P. W. E. Smith, E. Sorokin, and I. T. Sorokina. "Zscan characterization of the nonlinear refractive index of single crystal ZnSe in the 1.20-1.95 $\mathrm{um}$ wavelength range". In: Proceedings of SPIE $5971.59710 \mathrm{H}$ (2005). 
[111] D. T. F. Marple. "Refractive Index of ZnSe, ZnTe, and CdTe". In: Journal of Applied Physics 35.3 (1964), pages 539-542.

[112] D. E. Zelmon, D. L. Small, and R. Page. "Refractive-index measurements of undoped yttrium aluminum garnet from 0.4 to $5.0 \mu \mathrm{m}$ ". In: Applied Optics 37.21 (1998), pages 4933-4935.

[113] P. Samuel, T. R. Ensley, H. Hu, D. J. Hagan, E. W. Van Stryland, and R. Gaume. "Nonlinear refractive index measurement on pure and Nd doped YAG ceramic by dual arm Z-scan technique". In: AIP Conference Proceedings. Volume 1665. 060010. 2015.

[114] Irving H. Malitson. "Refraction and Dispersion of Synthetic Sapphire". In: Journal of the Optical Society of America 52.12 (1962), pages 1377-1379.

[115] A. Major, F. Yoshino, I. Nikolakakos, J. S. Aitchison, and P. W. E. Smith. "Dispersion of the nonlinear refractive index in sapphire". In: Optics Letters 29.6 (2004), pages 602-604.

[116] I. H. Malitson. "Interspecimen Comparison of the Refractive Index of Fused Silica". In: Journal of the Acoustical Society of America 55.10 (1965), pages 12051209.

[117] David Milam. "Review and assessment of measured values of the nonlinear refractive-index coefficient of fused silica". In: Applied Optics 37.3 (1998), pages $546-550$.

[118] M. J. Dodge. "Refractive properties of magnesium fluoride". In: Applied Optics 23.12 (1984), pages 1980-1985.

[119] R. DeSalvo, A. A. Said, D. J. Hagan, E. W. Van Stryland, and M. SheikBahae. "Infrared to ultraviolet measurements of two-photon absorption and $\mathrm{n}_{2}$ in wide bandgap solids". In: IEEE Journal of Quantum Electronics 32.8 (1996), pages 1324-1333.

[120] H. H. Li. "Refractive index of alkaline earth halides and its wavelength and temperature derivatives". In: Journal of Physical and Chemical Reference Data 9 (1980), pages 161-289.

[121] R. DeSalvo, M. Sheik-Bahae, A. A. Said, D. J. Hagan, and E. W. Van Stryland. "Z-scan measurements of the anisotropy of nonlinear refraction and absorption in crystals". In: Optics Letterse 18.3 (1993), pages 194-196.

[122] S. Das, G. C. Bhar, S. Gangopadhyay, and C. Ghosh. "Linear and nonlinear optical properties of $\mathrm{ZnGeP}_{2}$ crystal for infrared laser device applications: revisited". In: Applied Optics 42.21 (2003), pages 4335-4340.

[123] W. Li, Y. Li, and Y. Xu. "Measurements of nonlinear refraction in the mid-infrared materials $\mathrm{ZnGeP}_{2}$ and $\mathrm{AgGaS}_{2}$ ". In: Applied Physics B 123.82 (2017). 
[124] V. L. Kalashnikov. "Solid State Laser". In: edited by A. Al-Khursan. InTech, 2012. Chapter Chirped-Pulse Oscillators: Route to the Energy-Scalable Femtosecond Pulses, pages 145-184.

[125] P. N. Butcher and D. Cotter. The Elements of Nonlinear Optics. Cambridge University Press, 1990.

[126] T. Brabec and F. Krausz. "Nonlinear optical pulse propagation in the singlecycle regime". In: Physical Review Letters 78.17 (1997), pages 3282-3285.

[127] R. Trebino, K. W. DeLong, D. N. Fittinghoff, J. N. Sweetser, M. A. Krumbügel, and B. A. Richman. "Measuring ultrashort laser pulses in the timefrequency domain using frequency-resolved optical gating". In: Review of Scientific Instruments 68.9 (1998).

[128] A. Monmayrant, S. Weber, and B. Chatel. "A newcomer's guide to ultrashort pulse shaping and characterization". In: Journal of Physics B: Atomic, Molecular and Optical Physics 43.10 (2010), page 103001.

[129] R. Trebino. Frequency-Resolved Optical Gating: The Measurement of Ultrashort Laser Pulses. Springer-Verlag, 2000.

[130] A. Borzsonyi, A. P. Kovacs, and K. Osvay. "What we can learn about ultrashort pulses by linear optical methods". In: Applied Sciences 3.2 (2013), pages $515-544$.

[131] F. Mitschke, C. Mahnke, and A. Hause. "Soliton Content of Fiber-Optic Light Pulses". In: Applied Sciences 7.6 (2017), page 635.

[132] A. Hasegawa and M. Matsumoto. Optical Solitons in Fibers. Springer Series in Photonics. Springer, 2003.

[133] H. A. Haus. "Mode-Locking of Lasers". In: IEEE Journal of Selected Topics in Quantum Electronics 6.6 (2000), pages 1173-1185.

[134] W. H. Renninger, A. Chong, and F. W. Wise. "Are theorem and energy quantization for dissipative optical solitons". In: Journal of the Optical Society of America B 27.10 (2010), pages 1978-1982.

[135] E. P. Ippen. "Ultrafast Dynamics of Quantum Systems: Physical Processes and Spectroscopic Techniques". In: edited by B. Di Bartolo and G. Gambarota. Volume 372. Springer, 2002. Chapter Femtosecond Pulse Generation: Principles and Fiber Applications, pages 213-231.

[136] U. Keller. "Landolt-Bö rnstein - Group VIII/1B1". In: edited by G. Herziger, H. Weber, and R. Poprawe. Springer, 2007. Chapter Ultrafast solid-state lasers, pages $33-167$.

[137] J. Jasapara, W. Rudolph, V. L. Kalashnikov, D. O. Krimer, I. G. Poloyko, and M. Lenzner. "Automodulations in Kerr-lens mode-locked solid-state lasers". In: Journal of the Optical Society of America B 17.2 (2000), pages 319-326. 
[138] H. A. Haus. "Theory of mode locking with a fast saturable absorber". In: Journal of Applied Physics 46.7 (1975), pages 3049-3058.

[139] H. A. Haus, J. G. Fujimoto, and E. P. Ippen. "Structures for additive pulse mode locking". In: Journal of the Optical Society of America B 8.10 (1991), pages 2068-2076.

[140] E. Ding and J. N. Kutz. "Operating regimes, split-step modeling, and the Haus master mode-locking model". In: Journal of the Optical Society of America B 26.12 (2009), pages 2290-2300.

[141] G. Herink, B. Jalali, C. Ropers, and D. R. Solli. "Resolving the build-up of femtosecond mode-locking with single-shot spectroscopy at $90 \mathrm{MHz}$ frame rate". In: Nature Photonics 10 (2016), pages 321-326.

[142] R. I. Woodward. "Dispersion engineering of mode-locked fibre lasers". In: Journal of Optics 20 (2018), page 033002.

[143] M. Baumgartl, B. Ortaç, J. Limpert, and A. Tünnermann. "Impact of dispersion on pulse dynamics in chirped-pulse fiber lasers". In: Applied Physics B 107 (2012), pages 263-274.

[144] C. Spielmann, P. F. Curley, T. Brabec, and F. Krausz. "Ultrabroadband femtosecond lasers". In: IEEE Journal of Quantum Electronics 30.4 (1994), pages $1100-1114$.

[145] A. Fernandez, T. Fuji, A. Poppe, A. Fürbach, F. Krausz, and A. Apolonski. "Chirped-pulse oscillators: a route to high-power femtosecond pulses without external amplification". In: Optics Letters 29.12 (2004), pages 1366-1368.

[146] V. L. Kalashnikov, E. Podivilov, A. Chernykh, and A. Apolonski. "Chirpedpulse oscillators: theory and experiment". In: Applied Physics B 83 (2006), pages $503-510$.

[147] S. H. Cho et al. "Generation of 90-nJ pulses with a 4-MHz repetition-rate Kerr-lens mode-locked Ti: $\mathrm{Al}_{2} \mathrm{O}_{3}$ laser operating with net positive and negative intracavity dispersion". In: Optics Letters 26.8 (2001), pages 560-562.

[148] R. Haight and A. V. Carr. Industrial Applications of Ultrafast Lasers. Volume 11. Materials and Energy. WSPC, 2018.

[149] R. H. Page et al. "Cr" ${ }^{2+}$-Doped Zinc Chalcogenides as Efficient Widely Tunable Mid-Infrared Lasers". In: IEEE Journal of Quantum Electronics 33.4 (1997), pages 609-619.

[150] L. D. DeLoach, R. H. Page, D. Wilke, S. A. Payne, and W. F. Krupke. "Transition Metal-Doped Zinc Chalcogenides: Spectroscopy and Laser Demonstration of a New Class of Gain Media". In: IEEE Journal of Quantum Electronics 32.6 (1996), pages 885-895. 
[151] S. A. Payne and W. F. Krupke. "A Glimpse into the Laser-Crystal Ball". In: Optics and Photonics News 7.8 (1996), pages 31-35.

[152] J. Peppers, T. Konak, D. V. Martyshkin, V. Fedorov, and S. B. Mirov. "Spectroscopy and mid-IR lasing of $\mathrm{Cr}^{2+}$ ions in $\mathrm{ZnSe} / \mathrm{ZnS}$ crystals under visible excitation". In: Proceedings of SPIE - The International Society for Optical Engineering 8959 (2014).

[153] P. Schwendimann, E. Sigmund, and K. Zeile. "Model for laser action in vibronic systems". In: Physical Review A 37.8 (1988), pages 3018-3027.

[154] S. Kück. "Laser-related spectroscopy of ion-doped crystals for tunable solidstate lasers". In: Applied Physics B 72 (2001), pages 515-562.

[155] I. T. Sorokina. "Cr ${ }^{2+}$-doped II-VI materials for lasers and nonlinear optics". In: Optical Materials 26.4 (2004), pages 395-412.

[156] I. T. Sorokina et al. "Continuous-wave tunable $\mathrm{Cr}^{2+}: \mathrm{ZnS}$ laser". In: Applied Physics B: Lasers and Optics 74.6 (2002), pages 607-611.

[157] Landolt-Börnstein: Numerical Data and Functional Relationships in Science and Technology - New Series. Volume 41 Semiconductors, Subvolume B. Springer, 1999.

[158] P. F. Moulton. "Spectroscopic and laser characteristics of $\mathrm{Ti}: \mathrm{Al}_{2} \mathrm{O}_{3}$ ". In: Journal of the Optical Society of America B 3.1 (1986), pages 125-133.

[159] H. H. Kusuma, Z. Ibrahim, and M. K. Saidin. "Optical Energy Gap of Ti: $\mathrm{Al}_{2} \mathrm{O}_{3}$ Single Crystals". In: Journal of Applied Sciences 11.5 (2011), pages 888-891.

[160] I. T. Sorokina and E. Sorokin. "Femtosecond $\mathrm{Cr}^{2+}$-Based Lasers". In: IEEE Journal of Selected Topics in Quantum Electronics 21.1 (2015), page 1601519.

[161] S. B. Mirov, V. V. Fedorov, D. V. Martyshkin, L. S. Moskalev, M. S. Mirov, and V. P. Gapontsev. "Progress in mid-IR $\mathrm{Cr}^{2+}$ and $\mathrm{Fe}^{2+}$ doped II-VI materials and lasers [Invited]". In: Optical Materials Express 1.5 (2011).

[162] M. Baudrier-Raybaut, R. Haïdar, P. Kupecek, P. Lemasson, and E. Rosencher. "Random quasi-phase-matching in bulk polycrystalline isotropic nonlinear materials". In: Nature 432 (2004), pages 374-376.

[163] S. Vasilyev, M. Mirov, and V. Gapontsev. "High Power Kerr-Lens ModeLocked Femtosecond mid-IR Laser with Efficient Second Harmonic Generation in Polycrystalline $\mathrm{Cr}^{2+}: \mathrm{ZnS}$ and $\mathrm{Cr}^{2+}: \mathrm{ZnSe} "$. In: Advanced Solid State Lasers (ASSL) AM3A.3 (2014).

[164] S. Vasilyev, I. Moskalev, M. Mirov, V. Smolski, S. Mirov, and V. Gapontsev. "Mid-IR Kerr-lens mode-locked polycrystalline Cr:ZnS and Cr:ZnSe lasers with intracavity frequency conversion via random quasi-phase-matching". In: Proceedings of the SPIE 9731 (2016), 97310B. 
[165] M. N. Cizmeciyan, H. Cankaya, A. Kurt, and A. Sennaroglu. "Kerr-lens mode-locked femtosecond $\mathrm{Cr}^{2+}: \mathrm{ZnSe}$ laser at $2420 \mathrm{~nm}$ ". In: Optics Letters 34.20 (2009), pages 3056-3058.

[166] E. Sorokin and I. T. Sorokina. "Ultrashort-pulsed Kerr-lens modelocked Cr:ZnSe laser". In: CLEO Europe - EQEC CF1.3 (2009).

[167] S. Vasilyev et al. "Super-octave longwave mid-infrared coherent transients produced by optical rectification of few-cycle 2.5-um pulses". In: Optica 6.1 (2019), pages 111-114.

[168] S. B. Mirov, V. V. Fedorov, K. Graham, I. S. Moskalev, V. V. Badikov, and V. Panyutin. "Erbium fiber laser-pumped continuous-wave microchip $\mathrm{Cr}^{2+}: \mathrm{ZnS}$ and $\mathrm{Cr}^{2+}: Z n S e$ lasers". In: Optics Letters 27.11 (2002), pages 909-911.

[169] G. J. Wagner et al. "Continuous-wave broadly tunable $\mathrm{Cr}^{2+}$ :ZnSe laser". In: Optics Letters 24.1 (1999), pages 19-21.

[170] A. V. Podlipensky et al. "Efficient laser operation and continuous-wave diode pumping of $\mathrm{Cr}^{2+}$ :ZnSe single crystals". In: Applied Physics B 72.2 (2001), pages 253-255.

[171] I. Moskalev, V. Fedorov, S. Mirov, P. A. Berry, and K. Schepler. "12-Watt CW Polycrystalline $\mathrm{Cr}^{2+}: Z n S e$ Laser Pumped by Tm-fiber Laser". In: Advanced Solid-State Photonics. OSA. 2009.

[172] Y. Wu, F. Zhou, E. W. Larsen, F. Zhuang, Y. Yin, and Z. Chang. "Generation of few-cycle multi-millijoule $2.5 \mu \mathrm{m}$ pulses from a single-stage $\mathrm{Cr}^{2+}$ :ZnSe amplifier". In: Scientific Reports 10.7775 (2020).

[173] A. V. Podlipensky et al. "Pulsed laser operation of diffusion-doped $\mathrm{Cr}^{2+}: \mathrm{ZnSe}$ ". In: Optics Communications 167.1-6 (1999), pages 129-132.

[174] K. Graham et al. "Pulsed mid-IR $\mathrm{Cr}^{2+}: \mathrm{ZnS}$ and $\mathrm{Cr}^{2+}: Z n S e$ lasers pumped by Raman-shifted Q-switched neodymium lasers". In: Quantum Electronics 34.1 (2004), pages 8-14.

[175] A. Sennaroglu, A. O. Konca, and C. R. Pollock. "Continuous-Wave Power Performance of a 2.47- $\mu \mathrm{m} \mathrm{Cr}^{2+}$ :ZnSe Laser: Experiment and Modeling". In: IEEE Journal of Quantum Electronics 36.10 (2000), pages 1199-1205.

[176] P. A. Champert, S. V. Popov, and J. R. Taylor. "Efficient lasing of $\mathrm{Cr}^{2+}: \mathrm{ZnSe}$ at $2.2 \mathrm{\mu m}$ pumped by all-fibre-format seeded Raman source". In: Electronics Letters 38.10 (2002), pages 448-449.

[177] S. B. Mirov, V. V. Fedorov, D. Martyshkin, I. S. Moskalev, M. Mirov, and S. Vasilyev. "Progress in Mid-IR Lasers Based on Cr and Fe-Doped II-VI Chalcogenides". In: IEEE Journal of Selected Topics in Quantum Electronics 21.1 (2015), pages 292-310. 
[178] M. Ebrahim-Zadeh and I. T. Sorokina, editors. Mid-infrared Coherent Sources and Applications. Springer-Verlag, 2008.

[179] R. H. Page, J. A. Skidmore, K. I. Schaffers, R. J. Beach, S. A. Payne, and W. F. Krupke. "Demonstrations of diode-pumped and grating-tuned $\mathrm{ZnSe:Cr}{ }^{2+}$ lasers". In: OSA Trends in Optics and Photonics Series, Advanced Solid State Lasers 10 (1997), pages 208-210.

[180] I. S. Moskalev, V. V. Fedorov, and S. B. Mirov. "InP diode-pumped Cr:ZnS and Cr:ZnSe highly-efficient, widely-tunable, mid-IR lasers". In: Proceedings of SPIE 7578 (2010).

[181] G. J. Wagner, A. M. Schober, G. T. Bennett, D. L Bruns, J. H. Marquardt, and T. J. Carrig. "Multi-Watt Broadly-Tunable Diode-Pumped Cr:ZnSe Laser". In: Conference on Lasers and Electro-Optics 2012. CTu2D.2. Optical Society of America. OSA Technical Digest, 2012.

[182] E. Slobodtchikov and P. F. Moulton. "Progress in ultrafast Cr:ZnSe lasers". In: Lasers, Sources, and Related Photonic Devices. AW5A.4. Optical Society of America. OSA Technical Digest, 2012.

[183] E. Sorokin, N. Tolstik, K. I. Schaffers, and I. T. Sorokina. "Femtosecond SESAM-modelocked Cr:ZnS laser". In: Optics Express 20.27 (2012), pages 2894728952.

[184] J. Zhang et al. "49-fs Yb:YAG thin-disk oscillator with distributed Kerr-lens mode-locking". In: 2015 European Conference on Lasers and Electro-Optics European Quantum Electronics Conference (CLEO/Europe-EQEC) PD.A.1 (2015).

[185] M. Tokurakawa et al. "Diode-pumped 65 fs Kerr-lens mode-locked $\mathrm{Yb}^{3+}: \mathrm{Lu}_{2} \mathrm{O}_{3}$ and nondoped $\mathrm{Y}_{2} \mathrm{O}_{3}$ combined ceramic laser". In: Optics Letters 33.12 (2008), pages $1380-1382$.

[186] R. Phelan et al. "Optical Fiber Applications". In: edited by G. Huerta-Cuellar and R. Imani. IntechOpen. Chapter Mid-infrared InP-Based Discrete Mode Laser Diodes.

[187] R. Diehl, editor. High-Power Diode Lasers - Fundamentals, Technology, Applications. Volume 78. Springer, 2000.

[188] H. Sun. A Practical Guide to Handling Laser Diode Beams. 2nd edition. SpringerBriefs in Physics. Springer, 2015.

[189] M. Kot and K. Ždánský. "Measurement of radiative and nonradiative recombination rate in InGaAsP-InP LED's". In: IEEE Journal of Quantum Electronics 28.8 (1992), pages 1746-1750. 
[190] J. Piprek, J. K. White, and A. J. SpringThorpe. "What Limits the Maximum Output Power of Long-Wavelength AlGaInAs/InP Laser Diodes?" In: IEEE Journal of Quantum Electronics 38.9 (2002), pages 1253-1259.

[191] K. Qing et al. "Fabrication and optimization of 1.55- $\mu \mathrm{m}$ InGaAsP/InP high-power semiconductor diode laser". In: Journal of Semiconductors 36.9 (2015).

[192] C. C. Harb, T. C. Ralph, E. H. Huntington, D. E. McClelland, H.-A. Bachor, and I. Freitag. "Intensity-noise dependence of Nd:YAG lasers on their diodelaser pump source". In: Journal of the Optical Society of America B 14.11 (1997), pages 2936-2945.

[193] Y. Wang, T. T. Fernandez, N. Coluccelli, A. Gambetta, P. Laporta, and G. Galzerano. "47-fs Kerr-lens mode-locked Cr:ZnSe laser with high spectral purity". In: Optics Express 25.21 (2017), pages 25193-25200.

[194] S. Yang, E. A. Ponomarev, and X. Bao. "Experimental study on relaxation oscillation in a detuned FM harmonic mode-locked Er-doped fiber laser". In: Optics Communications 245.1-6 (2005), pages 371-376.

[195] W.-N. Zhang et al. "A Compact Low Noise Single Frequency Linearly Polarized DBR Fiber Laser at 1550 nm". In: Chinese Physics Letters 29.8 (2012), page 084205 .

[196] Y. Cheng, J. T. Kringlebotn, W. H. Loh, R. I. Laming, and D. N. Payne. "Stable single-frequency traveling-wave fiber loop laser with integral saturableabsorber-based tracking narrow-band filter". In: Optics Letters 20.8 (1995), pages $875-877$.

[197] G. Zhu, Q. Wang, H. Dong, and N. K. Dutta. "Fiber laser relaxation oscillation noise suppression through the use of self-biased intracavity loss modulator". In: Journal of Applied Physics 96.4 (2004), pages 1790-1793.

[198] H. Wang, L. Wang, D. S. Jakob, and X. G. Xu. "Tomographic and multimodal scattering-type scanning near-field optical microscopy with peak force tapping mode". In: Nature Communications 9.2005 (2018).

[199] D. G. Hall. "Optimum mode size criterion for low-gain lasers". In: Applied Optics 20.9 (1981), pages 1579-1583.

[200] D. Kopf, J. Aus der Au, U. Keller, G. L. Bona, and P. Roentgen. "400-mW continuous-wave diode-pumped Cr:LiSAF laser based on a power-scalable concept". In: Optics Letters 20.17 (1995), pages 1782-1784.

[201] D. Kopf et al. "High-average-power diode-pumped femtosecond Cr:LiSAF lasers". In: Applied Physics B 65 (1997), page 235.243.

[202] F. Krausz, J. Zehetner, T. Brabec, and E. Wintner. "Elliptic-mode cavity for diode-pumped lasers". In: Optics Letters 16.19 (1991), pages 1496-1498. 
[203] P. Laporta and M. Brussard. "Design Criteria for Mode Size Optimization in Diode-Pumped Solid-State Lasers". In: IEEE Journal of Quantum Electronics 27.10 (1991), pages 2319-2326.

[204] F. Salin and J. Squier. "Geometrical optimization of longitudinally pumped solid-state lasers". In: Optics Communications 86 (1991), pages 397-400.

[205] F. Sanchez, M. Brunel, and K. Aït-Ameur. "Pump-saturation effects in endpumped solid-state lasers". In: Journal of the Optical Society of America B 15.9 (1998), pages 2390-2394.

[206] S. Backus, M. Kirchner, C. Durfee, M. Murnane, and H. Kapteyn. "Direct diode-pumped Kerr Lens 13 fs Ti:sapphire ultrafast oscillator using a single blue laser diode". In: Optics Express 25.11 (2017), pages 12469-12477.

[207] C. G. Durfee et al. "Direct diode-pumped Kerr-lens mode-locked Ti:sapphire laser". In: Optics Express 20.13 (2012), pages 13677-13683.

[208] K. Gürel et al. "Green diode-pumped femtosecond Ti:sapphire laser with up to $450 \mathrm{~mW}$ average power". In: Optics Express 20.23 (2015), pages 3004330048.

[209] N. Nagl, S. Gröbmeyer, V. Pervak, F. Krausz, O. Pronin, and K. Mak. "Directly diode-pumped, Kerr-lens mode-locked, few-cycle Cr:ZnSe oscillator". In: Optics Express 27.17 (2019), pages 24445-24454.

[210] Lasers and laser-related equipment - Test methods for laser beam widths, divergence angles and beam propagation ratios. ISO Standard 11146. 2005.

[211] A. E. Siegman. "Defining, measuring, and optimizing laser beam quality". In: Proceedings of SPIE 1868 (1993).

[212] X. Wang, G. Hu, Y. Li, and J. Yao. "Numerical analysis of beam parameters and stability regions in a folded or ring cavity". In: Journal of the Optical Society of America A 11.8 (1994), pages 2265-2270.

[213] S. N. B. Tushar, S. D. Nath, J. Akhtar, and M. I. Reja. "Modeling and Analysis of Z Folded Solid State Laser Cavity with Two Curved Mirrors". In: International Journal of Microwave and Optical Technology 13.3 (2018), pages 244-253.

[214] S. B. Mirov et al. "Diode and fibre pumped $\mathrm{Cr}^{2+}: \mathrm{ZnS}$ mid-infrared external cavity and microchip lasers". In: IEE Proceedings - Optoelectronics 150.4 (2003), pages 340-345.

[215] T. Brabec, F. Curley, C. Spielmann, E. Wintner, and J. A. Schmidt. "Hardaperture Kerr-lens mode locking". In: Journal of the Optical Society of America B 10.6 (1993), pages 1029-1034.

[216] N. Tolstik, E. Sorokoin, and I. T. Sorokina. "Kerr-lens mode-locked Cr:ZnS laser". In: Optics Letters 38.3 (2013), pages 299-301. 
[217] H. A. Haus, J. G. Fujimoto, and E. P. Ippen. "Analytic Theory of Additive Pulse and Kerr Lens Mode Locking". In: IEEE Journal of Quantum Electronics 28.10 (1992), pages 2086-2096.

[218] Robert Szipöcs, Kárpát Ferencz, Christian Spielmann, and Ferenc Krausz. "Chirped multilayer coatings for broadband dispersion control in femtosecond lasers". In: Optics Letters 19.3 (1994), pages 201-203.

[219] U. Morgner et al. "Ultrabroadband double-chirped mirror pairs covering one octave of bandwidth". In: Conference on Lasers and Electro-Optics. 2000, CThE5.

[220] F. X. Kärtner et al. "Design and fabrication of double-chirped mirrors". In: Optics Letters 22.11 (1997), pages 831-833.

[221] R. Szipöcs et al. "Negative dispersion mirrors for dispersion control in femtosecond lasers: chirped dielectric mirrors and multi-cavity Gires-Tournois interferometers". In: Applied Physics B 70 (2000), S51-S57.

[222] B. Golubovic et al. "Double Gires-Tournois interferometer negative-dispersion mirrors for use in tunable mode-locked lasers". In: Optics Letters 25.4 (2000), pages 275-277.

[223] V. Pervak, T. Amotchkina, Q. Wang, O. Pronin, K. Mak, and M. Trubetskov. "2/3 octave $\mathrm{Si} / \mathrm{SiO}_{2}$ infrared dispersive mirrors open new horizons in ultrafast multilayer optics". In: Optics Express 27.1 (2019), pages 55-62.

[224] T. Brabec, Ch. Spielmann, P.F.Curley, and F. Krausz. "Kerr lens mode locking". In: Optics Letters 17.18 (1992), pages 1292-1294.

[225] H. Kogelnik and T. Li. "Laser Beams and Resonators". In: Applied Optics 5.10 (1966), pages 1550-1567.

[226] S. T. Cundiff, J. M. Soto-Crespo, and N. Akhmediev. "Experimental Evidence for Soliton Explosions". In: Physical Review Letters 88.7 (2002), page 073903.

[227] A. Müller et al. "Frequency-doubled DBR-tapered diode laser for direct pumping of Ti:sapphire lasers generating sub-20 fs pulses". In: Optics Express 19.13 (2011), pages 12156-12163.

[228] M. L. Dennis and I. N. Duling III. "Experimental Study of Sideband Generation in Femtosecond Fiber Lasers". In: IEEE Journal of Quantum Electronics 30.6 (1994), pages 1469-1477.

[229] G. P. Agrawal and N. K. Dutta. "Semiconductor Lasers". In: Springer US, 1993. Chapter Noise characteristics, pages 261-269.

[230] C. H. Henry and R. F. Kazarinov. "Quantum noise in photonics". In: Reviews of Modern Physics 68.3 (1996), pages 801-853. 
[231] Optics and photonics - Lasers and laser-related equipment - Test methods for laser beam power, energy and temporal characteristics. ISO Standard 11554. 2017.

[232] R. Paschotta et al. "Relative timing jitter measurements with an indirect phase comparison method". In: Applied Physics B 80 (2005), pages 185-192.

[233] Gregory E. Obarski and Jolene D. Splett. "Transfer standard for the spectral density of relative intensity noise of optical fiber sources near $1550 \mathrm{~nm}$ ". In: Journal of the Optical Society of America B 18.6 (2001), pages 750-761.

[234] M. Tawfieq, A. Kragh Hansen, O. Bjarlin Jensen, D. Marti, B. Sumpf, and P. E. Andersen. "Intensity noise transfer through a diode-pumped titanium sapphire laser system". In: IEEE Journal of Quantum Electronics 54.1 (2018), page 1700209.

[235] R. P. Scott, C. Langrock, and B. H. Kolner. "High-Dynamic-Range Laser Amplitude and Phase Noise Measurement Techniques". In: IEEE Journal on Selected Topics in Quantum Electronics 7.4 (2001), pages 641-655.

[236] F. Seifert, P. Kwee, M. Heurs, B. Willke, and K. Danzmann. "Laser power stabilization for second-generation gravitational wave detectors". In: Optics Letters 31.13 (2006), pages 2000-2002.

[237] Y. Wang, J. Fonseca-Campos, W.-G. Liang, C.-Q. Xu, and I. Vargas-Baca. "Noise analysis of Second-Harmonic Generation in Undoped and $\mathrm{MgO}-$ Doped Periodically Poled Lithium Niobate". In: Advances in OptoElectronics 2008.428971 (2008).

[238] F. N. Hooge, T. G. M. Kleinpenning, and L. K. J. Vandamme. "Experimental studies on 1/f noise". In: Reports on Progress in Physics 44 (1981), pages 479531.

[239] P. Dutta and P. M. Horn. "Low-frequency fluctuations in solids: 1/f noise". In: Reviews of Modern Physics 53.3 (1981), pages 497-516.

[240] M. S. Keshner. "1/f Noise". In: Proceedings of the IEEE 70.3 (1982), pages 212218.

[241] O. Llopis, S. Azaizia, K. Saleh, A. A. Slimane, and A. Fernandez. "Photodiode $1 / \mathrm{f}$ noise and other types of less known baseband noises in optical telecommunications devices". In: 22nd International Conference on Noise and Fluctuations (ICNF) 13710909 (2013).

[242] L. F. Mollenauer and R. H. Stolen. "The soliton laser". In: Optics Letters 9.1 (1984), pages 13-15.

[243] Q. Wang et al. "Complete Mid-Infrared Coverage (2-20 $\mu \mathrm{m})$ via Cascaded Parametric Processes". In: Laser Congress 2018 (ASSL) ATu6A.4 (2018). 
[244] J. M. Dudley. "Supercontinuum generation in photonic crystal fiber". In: Reviews of modern physics 78.4 (2006), pages 1135-1184.

[245] D. Grassani et al. "Mid infrared gas spectroscopy using efficient fiber laser driven photonic chip-based supercontinuum". In: Nature Communications 10.1553 (2019).

[246] S. Vasilyev, I. Moskalev, M. Mirov, V. Smolski, S. Mirov, and V. Gapontsev. "Ultrafast middle-IR lasers and amplifiers based on polycrystalline Cr:ZnS and Cr:ZnSe". In: Optics Materials Express 7.7 (2017), pages 2636-2650.

[247] S. Backus et al. "Direct diode pumped Ti:sapphire ultrafast regenerative amplifier system”. In: Optics Express 25.4 (2017), pages 3666-3674.

[248] N. Sugiyama, H. Tanaka, and F. Kannari. "Mode-locked Ti:sapphire laser oscillators pumped by wavelength-multiplexed laser diodes". In: Japanese Journal of Applied Physics 57.5 (2018), page 052701.

[249] M. Byrd et al. "Wavelength Selection and Polarization Multiplexing of Blue Laser Diodes". In: IEEE Photonics Technology Letters 27.20 (2015), pages 2166-2169.

[250] U. Keller. "Ultrafast All-Solid-State Laser Technology". In: Applied Physics B 58 (1994), pages 347-363.

[251] F. X. Kärtner, J. Aus der Au, and U. Keller. "Mode-Locking with Slow and Fast Saturable Absorbers - What's the Difference?" In: IEEE Journal of Selected Topcis in Quantum Electronics 4.2 (1998), pages 159-168.

[252] F. X. Kärtner, I. D. Jung, and U. Keller. "Soliton Mode-Locking with Saturable Absorbers". In: IEEE Journal of Selected Topics in Quantum Electronics 2.3 (1996), pages 540-556.

[253] J. Aus der Au, D. Kopf, F. Morier-Genoud, M. Moser, and U. Keller. "60-fs pulses from a diode-pumped Nd:glass laser". In: Optics Letters 22.5 (1997), pages 307-309.

[254] V. L. Kalashnikov, E. Sorokin, and I. T. Sorokina. "Multipulse Operation and Limits of the Kerr-Lens Mode-Locking Stability". In: IEEE Journal of Quantum Electronics 39.2 (2003), pages 323-336.

[255] Y. W. Lee, J. H. Yi, Y. H. Cha, Y. J. Rhee, B. C. Lee, and B. D. Yoo. "Numerical analysis of soft-aperture Kerr-lens mode locking in Ti:Sapphire laser cavities by using nonlinear ABCD matrices". In: Journal of the Korean Physical Society 46.5 (2005), pages 1131-1136.

[256] V. Magni, G. Cerullo, and S. De Silvestri. "ABCD matrix analysis of propagation of gaussian beams through Kerr media". In: Optics Communications 96.4-6 (1993), pages 348-355. 
[257] E. J. Grace, A. Ritsataki, P. M. W. French, and G. H. C. New. "New optimization criteria for slit-apertured and gain-apertured KLM all-solidstate lasers". In: Optics Communications 183 (2000), pages 249-264.

[258] M. J. P. Dymott and A. I. Ferguson. "Self-mode-locked diode-pumped Cr:LiSAF laser". In: Optics Letters 19.23 (1994), pages 1988-1990.

[259] I. T. Sorokina, E. Sorokin, E. Wintner, A. Cassanho, H. P. Jenssen, and R. Szipöcs. "Sub-20 fs pulse generation from the mirror dispersion controlled Cr:LiSGaF and Cr:LiSAF lasers". In: Applied Physics B 65 (1997), pages 245253.

[260] L. Xu et al. "High-power sub-10-fs Ti:sapphire oscillators". In: Applied Physics B 65.2 (1997), pages 151-159.

[261] G. Cerullo, S. De Silvestri, and V. Magni. "Self-starting Kerr-lens mode locking of a Ti:sapphire laser". In: Optics Letters 19.14 (1994), pages 10401042.

[262] P. Xia, M. Kuwata-Gonokami, and K. Yoshioka. "Geometrical analysis of Kerr-lens mode-locking for high-peak-power ultrafast oscillators". In: Japanese Journal of Applied Physics 59.062002 (2020).

[263] F. Salin and J. Squier. "Gain guiding in solid-state lasers". In: Optics Letters 17.19 (1992), pages 1352-1354.

[264] A. Yariv. "Energy and Power Considerations in Injection and Optically Pumped Lasers". In: Proceedings of the IEEE 51.12 (1963), pages 1723-1731.

[265] N. Hodgson and H. Weber. "Laser Resonators and beam propagation". In: volume 108. Springer Series in Optical Sciences. Springer New York, 2005. Chapter Output power of laser resonators, pages 393-412.

[266] Nathalie Nagl, Sebastian Gröbmeyer, Markus Potzlberger, Vladimir Pervak, Ferenc Krausz, and Ka Fai Mak. "Directly Diode-Pumped Few-Optical-Cycle $\mathrm{Cr}: Z n S$ Laser at $800 \mathrm{~mW}$ of Average Power". In: Conference on Lasers and Electro-Optics. Optical Society of America, 2020, SF3H.5.

[267] R. Salem et al. "Mid-infrared supercontinuum generation spanning 1.8 octaves using step-index indium fluoride fiber pumped by a femtosecond fiber laser near 2 um". In: Optics Express 23.24 (2015), pages 30592-30602.

[268] Y. Tang, L. G. Wright, K. Charan, T. Wang, C. Xu, and F. W. Wise. "Generation of intense 100 fs solitons tunable from 2 to $4.3 \mu \mathrm{m}$ in fluoride fiber". In: Optica 3.9 (2016), pages 948-951.

[269] H. Ren et al. "Low-loss silicon core fibre platform for mid-infrared nonlinear photonics". In: Light: Science $\&$ Applications 8.105 (2019). 
[270] T. S. Saini, T. H. Tuan, T. Suzuki, and Y. Ohishi. "Coherent Mid-IR Supercontinuum Generation using Tapered Chalcogenide Step-Index Optical Fiber: Experiment and modelling". In: Scientific Reports 10.2236 (2020).

[271] T. P. Butler et al. "Watt-scale 50-MHz source of single-cycle waveform-stable pulses in the molecular fingerprint region". In: Optics Letters 44.7 (2019), pages $1730-1733$.

[272] V. Smolski et al. "Half-Watt average power femtosecond source spanning 3-8 $\mu \mathrm{mu}$ based on subharmonic generation in GaAs". In: Applied Physics B 124.101 (2018).

[273] C. Homann, M. Bradler, M. Förster, P. Hommelhoff, and E. Riedle. "Carrierenvelope phase stable sub-two-cycle pulses tunable around $1.8 \mu \mathrm{m}$ at 100 kHz". In: Optics Letters 37.10 (2012), pages 1673-1675.

[274] H. R. Telle, G. Steinmeyer, A. E. Dunlop, J. Stenger, D. H. Sutter, and U. Keller. "Carrier-envelope offset phase control: A novel concept for absolute optical frequency measurement and ultrashort pulse generation". In: Applied Physics B 69 (1999), pages 327-332.

[275] L. Xu, Ch. Spielmann, A. Poppe, T. Brabec, F. Krausz, and T. W. Hänsch. "Route to phase control of ultrashort light pulses". In: Optics Letters 21.24 (1996), pages 2008-2010.

[276] G. Cerullo, A. Baltuska, O. D. Mücke, and C. Vozzi. "Few-optical-cycle light pulses with passive carrier-envelope phase stabilization". In: Laser\&̈Photonics Reviews 5.3 (2011), pages 323-351.

[277] Y.-S. Lee. Principles of Terahertz Science and Technology. Springer US, 2009.

[278] V. L. Kalashnikov and E. Sorokin. "Soliton absorption spectroscopy". In: Physical Review A 81.033840 (2010).

[279] M. Gebhardt et al. "Impact of atmospheric molecular absorption on the temporal and spatial evolution of ultra-short optical pulses". In: Optics Express 23.11 (2015), pages 13776-13787.

[280] Hisaaki Kudo, editor. Radiation Applications. Volume 7. An Advanced Course in Nuclear Engineering. Springer Singapore, 2018.

[281] J. W. Goodman. "Introduction to Fourier Optics". In: 3rd edition. Roberts and Company Publishers, 2005. Chapter Analog Optical Information Processing, pages 219-290.

[282] A. P. Yelisseyev, L. I. Isaenko, and M. K. Starikova. "Optical study of defects in lithium iodate $\alpha$ - $\mathrm{LiO}_{3}$ ". In: Journal of the Optical Society of America B 29.6 (2012), pages 1430-1435. 
[283] N. Tolstik, E. Sorokin, and I. T. Sorokina. "Graphene mode-locked Cr:ZnS laser with 41 fs pulse duration". In: Optics Express 22.5 (2014), pages 55645571 .

[284] V. Pervak, O. Razskazovskaya, I. B. Angelov, K. L. Vodopyanov, and M. Trubetskov. "Dispersive mirror technology for ultrafast lasers in the range 220-4500 nm". In: Advanced Optical Technologies 3.1 (2014), pages 55-63.

[285] V. Laude and P. Tournois. "Chirped-mirror-pairs for ultra-broadband dispersion control". In: Conference on Lasers and Electro-Optics. Optical Society of America, 1999, CTuR4.

[286] F. X. Kärtner et al. "Ultrabroadband double-chirped mirror pairs for generation of octave spectra". In: Journal of the Optical Society of America B 18.6 (2001), pages 882-885.

[287] V. Pervak, A. V. Tikhonravov, M. K. Trubetskov, S. Naumov, F. Krausz, and A. Apolonski. "1.5-octave chirped mirror for pulse compression down to sub-3 fs". In: Applied Physics B 87 (2007), pages 5-12.

[288] S. Rausch et al. "Controlled waveforms on the single-cycle scale from a femtosecond oscillator". In: Optics Express 16.13 (2008), pages 9739-9745.

[289] M. Y. Sander, J. Birge, A. Benedick, H. M. Crespo, and F. X. Kärtner. "Dynamics of dispersion managed octave-spanning titanium:sapphire lasers". In: Journal of the Optical Society of America B 26.4 (2009), pages 743-749.

[290] S. Vasilyev, I. Moskalev, M. Mirov, S. Mirov, and V. Gapontsev. "Three optical cycle mid-IR Kerr-lens mode-locked polycrystalline $\mathrm{Cr}^{2+}: \mathrm{ZnS}$ laser". In: Optics Letters 40.21 (2015), pages 5054-5057.

[291] J. Brons et al. "Energy scaling of Kerr-lens mode-locked thin-disk oscillators". In: Optics Letters 39.22 (2014), pages 6442-6445.

[292] W. Tian et al. "Diode-pumped power scalable Kerr-lens mode-locked Yb:CYA laser". In: Photonics Research 6.2 (2018), pages 127-131.

[293] W. Tian et al. "Diode-pumped high-power sub-100 fs Kerr-lens mode-locked Yb:CaYAlO4 laser with 1.85 MW peak power". In: Optics Express 27.15 (2019), pages 21448-21454.

[294] R. Akbari and A. Major. "Kerr-lens mode locking of a diode-pumped Yb:KGW laser using an additional intracavity Kerr medium". In: Laser Physics Letters 15.8 (2018), page 085001.

[295] X. Han and H. Zeng. "Kerr-lens mode-locked Ti:sapphire laser with an additional intracavity nonlinear medium". In: Optics Express 16.23 (2008), pages $18875-18880$. 
[296] S. H. Cho, B. E. Bouma, E. P. Ippen, and J. G. Fujimoto. "Low-repetition-rate high-peak-power Kerr-lens mode-locked Ti: $\mathrm{Al}_{2} \mathrm{O}_{3}$ laser with a multiple-pass cavity". In: Optics Letters 24.6 (1999), pages 417-419.

[297] A. M. Kowalevicz, A. Sennaroglu, A. T. Zare, and J. G. Fujimoto. "Design principles of q-preserving multipass-cavity femtosecond lasers". In: Journal of the Optical Society of America B 23.4 (2006), pages 760-770.

[298] D. H. Song, W. B. Cho, H. W. Lee, D. H. Shin, D.-K. Ko, and M. Y. Jung. "A compact Kerr-lens mode-locked Ti:sapphire oscillator with 330 nJ soliton-like pulses". In: Laser Physics Letters 10.065003 (2013).

[299] L.-J. Chen, M. Y. Sander, and F. X. Kärtner. "Kerr-lens mode locking with minimum nonlinearity using gain-matched output couplers". In: Optics Letters 35.17 (2010), pages 2916-2918.

[300] C. Cihan et al. "Gain-Matched Output Couplers for Efficient Kerr-Lens ModeLocking of Low-Cost and High-Peak Power Cr:LiSAF Lasers". In: IEEE Journal of Selected Topics in Quantum Electronics 21.1 (2014), pages 94-105.

[301] N. A. Tolstik, A. Pospischil, E. Sorokin, and T. Sorokina. "Graphene Modelocked Cr:ZnS Chirped-pulse oscillator". In: Optics Express 22.6 (2014), pages 7284-7289.

[302] N. Tolstik, C. S. J. Lee, E. Sorokin, and I. T. Sorokina. "8.6 MHz Extended Cavity Cr:ZnS Chirped-pulse Oscillator". In: Conference on Lasers and Electro-Optics. 2018, SF1N.2.

[303] A. G. Astill. "Material figures of merit for non-linear optics". In: Thin Solid Films 204.1 (1991), pages 1-17.

[304] K. L. Vodopyanov, F. Ganikhanov, J. P. Maffetone, I. Zwieback, and W. Ruderman. "ZnGeP 2 optical parametric oscillator with 3.8-12.4- $\mu$ m tunability". In: Optics Letters 25.11 (2000), pages 841-843.

[305] A. Hemming et al. "99 W mid-IR operation of a ZGP OPO at $25 \%$ duty cycle". In: Optics Express 21.8 (2013), pages 10062-10069.

[306] D. Sanchez et al. "7 $\mu \mathrm{m}$, ultrafast, sub-millijoule-level mid-infrared optical parametric chirped pulse amplifier pumpet at 2 um”. In: Optica 3.2 (2016), pages $147-150$.

[307] C. Qian et al. "11.4 W long-wave infrared source based on $\mathrm{ZnGeP}_{2}$ optical parametric amplifier". In: Optics Express 26.23 (2018), pages 30195-30201.

[308] S. Vasilyev et al. "Multi-octave infrared femtosecond continuum generation in Cr:ZnS-GaSe and Cr:ZnS-ZGP tandems". In: Nonlinear Frequency Generation and Conversion: Materials and Devices XIX. Edited by Peter G. Schunemann and Kenneth L. Schepler. Volume 11264. International Society for Optics and Photonics. SPIE, 2020, pages 13-24. 
[309] S. Vasilyev et al. "Multi-octave visible to long-wave IR femtosecond continuum generated in Cr:ZnS-GaSe tandem”. In: Optics Express 27.11 (2019), pages 16405-16413.

[310] S. Polyakov, F. Yoshino, and G. Stegeman. "Interplay between self-focusing and high-order multiphoton absorption". In: Journal of the Optical Society of America B 18.12 (2001), pages 1891-1895.

[311] K. L. Vodopyanov. Laser-based Mid-infrared Sources and Applications. Wiley, 2020.

[312] W. G. Wagner, H. A. Haus, and J. H. Marburger. "Large-Scale Self-Trapping of Optical Beams in the Paraxial Ray Approximation". In: Physical Review 175.1 (1968), pages 256-266.

[313] A. Couairon and A. Mysyrowicz. "Progress in Ultrafast Intense Laser Science". In: volume 84. Springer Series in Chemical Physics. Springer, 2006. Chapter Femtosecond Filamentation in Air, pages 235-258.

[314] U. Willer, M. Saraji, A. Khorsandi, P. Geiser, and W. Schade. "Near- and midinfrared laser monitoring of industrial processes, environment and security applications". In: Optics and Lasers in Engineering 44.7 (2006), pages 699710.

[315] I. Pupeza et al. "Field-Resolved Spectroscopy in the Molecular Fingerprint Region". In: Conference on Lasers and Electro-Optics Europe 63 European Quantum Electronics Conference (CLEO/Europe-EQEC) CH-2.4 (2017).

[316] P. Lasch and J. Kneipp. Biomedical Vibrational Spectroscopy. Wiley, 2008.

[317] N. R. Newbury, I. Coddington, and W. Swann. "Sensitivity of coherent dual-comb spectroscopy". In: Optics Express 18.8 (2010), pages 7929-7945.

[318] B.-H. Chen, E. Wittmann, Y. Morimoto, P. Baum, and E. Riedle. "Octavespanning single-cycle middle-infrared generation through optical parametric amplification in $\mathrm{LiGaS}_{2}$ ". In: Optics Express 27.15 (2019), pages 21306-21318.

[319] S. Gröbmeyer et al. "Self-compression at $1 \mu \mathrm{m}$ wavelength in all-bulk multipass geometry". In: Applied Physics B 126.159 (2020).

[320] M. Seidel, G. Arisholm, J. Brons, V. Pervak, and O. Pronin. "All solidstate spectral broadening: an average and peak power scalable method for compression of ultrashort pulses". In: Optics Express 24.9 (2016), pages 94129428.

[321] R. Šuminas, A. Marcinkevičiūtè, G. Tamošauskas, and A. Dubietis. "Even and odd harmonics-enhanced supercontinuum generation in zinc-blende semiconductors". In: J. Opt. Soc. Am. B 36.2 (2019), A22-A27. 


\section{ACKNOWLEDGEMENTS}

At the end of my thesis, I would like to take the opportunity to express my heartfelt gratitude to all those people who, one way or another, have supported me during my time as a $\mathrm{PhD}$ student:

- First of all, I would like to thank my PhD advisor, Ferenc Krausz, for his continuous encouragement and support on the project. I really enjoyed to be part of the big Attoworld research group and it was very enriching to work together with colleagues from so many different fields of laser physics. Moreover, I would like to thank him for the inspiration that he gave me based on the concept of a Just Cause.

- A very special thanks goes out to my group leader Kafai Mak, who has teached me the secrets behind Cr:ZnS/ZnSe laser systems based on his own fiber-laserpumped oscillator. He always had an open ear for my ideas and concerns, and I cannot say whether the progress of my experimental research would have been that fast without his mental and also scientific support - ranging from nonlinear optics to python programming and presentation skills. In addition, I also want to thank Oleg Pronin, who was my former group leader during the first year of my $\mathrm{PhD}$, for all his support. I am especially grateful that he and Ferenc Krausz recommended me to the Cusanuswerk.

- Thanks to my (former) team members of the "AG HFS" group and officemates around Sebastian Gröbmeyer, Markus Pötzlberger, Kilian Fritsch, Jinwei Zhang, Qing Wang, Philipp Steinleitner, Aleksandar Sebesta, Shizhen Qu, Maciej Kowalczyk, and Naeem Ahmad. I really enjoyed working together with all of you! Moreover, it was a great pleasure to join our daily walk to our canteen next door and the yearly Christmas activities, ranging from lasertag and go-carting to skiing and city walks. Though I was surrounded by male colleagues only, it was so much fun with you, guys!

- Also, I want to thank all my colleagues around Ioachim Pupeza's and Mihaela Zigman's group for the regular exchange of knowledge during our regular team meetings, and also thanks for lending experimental equipment to us. In particular, I am thankful to Alexander Weigel and Marinus Huber for the useful discussions on amplitude noise measurements. 
- The generation of few-cycle pulses from Cr:ZnS/ZnSe oscillators would not have been possible without the dedicated multi-layer optics provided by Vladimir Pervak and his team. It was really helpful to have you sitting just a couple of rooms away for discussing all the various customized mirror designs that emerged during my time as a $\mathrm{PhD}$.

- Particular thanks also goes to all the organizers of the International Max Planck Research School of Advanced Photon Science (IMPRS-APS). I really enjoyed being part of the graduate school, especially because of the great training opportunities through seminars, workshops and the block lectures. Also, the annual meetings at Ringberg Castle were legendary!

- In addition, I would like to acknowledge the financial and immaterial support that I received as part of my doctoral scholarship by the Bischöfliche Studienförderung Cusanuswerk. It was a great honour for me to meet so many inspiring people from all academic disciplines and I will always remember the discussions that we had and the time that we spent during our meetings.

- A special thanks goes out to all the administrative and technical staff at our institute, in particular Klaus Franke, Katharina Adler, Martin Groß, Jana Gerding, Kerstin Schmidt-Buchmann, Hans-Friedrich Wirth, Florian Saran, Thorsten Naeser, and the mechanical workshop.

- I would also like to thank Prof. Dr. Ursula Keller for offering to review my thesis.

- I also want to thank all the people who contributed to this thesis in one way or another but whom I forgot to mention.

- Ein ganz herzlicher Dank gilt schließlich auch meiner Familie, insbesondere meinen Eltern und Großeltern sowie meinen Geschwistern, dass sie mich zu jeder Zeit unterstützt haben. Ohne euch wäre dieser Weg so bestimmt nicht möglich gewesen! Auch möchte ich mich bei Markus bedanken, der mir als Partner in jeder Situation zur Seite stand und immer ein offenes Ohr für mich hatte. 


\section{DECLARATION}

Hiermit erkläre ich, die vorliegende Arbeit selbständig verfasst zu haben und keine anderen als die in der Arbeit angegebenen Quellen und Hilfsmittel benutzt zu haben.

Hereby I assure that I did this thesis independently and used no other than the stated sources and aids.

München, 30. Oktober 2020

Nathalie Nagl 